\title{
Very Long Chain Fatty Acid Metabolism Is Required In Acute Myeloid Leukemia
}

\author{
By \\ Matthew Tcheng
}

\author{
A Thesis \\ presented to \\ The University of Guelph
In partial fulfilment of requirements
for the degree of
Doctor of Philosophy
in \\ Food Science
}

Guelph, Ontario, Canada

(C) Matthew Tcheng, August, 2021 


\begin{abstract}
VERY LONG CHAIN FATTY ACID METABOLISM IS REQUIRED IN ACUTE MYELOID LEUKEMIA
\end{abstract}

Matthew Tcheng University of Guelph, 2021
Advisor:

Professor P. A. Spagnuolo

Acute myeloid leukemia (AML) is a devastating blood cancer characterized by the uncontrolled proliferation of abnormal myeloid blasts that fail to differentiate into functional blood cells. AML therapy has remained virtually unchanged for the past four decades and triggers significant co-morbidities, preventing dose escalation in mature AML patients. Compared to normal hematopoietic cells, the AML population exhibited an abnormal mitochondrial phenotype sustained by an increased reliance on fatty acid oxidation (FAO) coupled to oxidative phosphorylation (OXPHOS), conferring a pro-survival advantage against induction chemotherapeutics. Anti-AML FAO inhibitors have not been approved for clinical use or induced unacceptable liver toxicities. The lack of potential compounds for further clinical development underscore the need to identify novel compounds that inhibit FAO in AML and are well-tolerated by the normal hematopoietic population.

In chapters 4,5 , and 6 , this thesis elucidated the mechanism of action of avocadyne (AYNE), a novel FAO inhibitor that selectively killed leukemia cells while sparing normal blood cells. In chapter 4, AYNE was identified as the active component of avocatin-B (avo-B), a 1:1 mixture of fatty alcohols AYNE and avocadene (AENE). In chapter 5, the very long chain acylCoA dehydrogenase (VLCAD), an intramitochondrial FAO enzyme that is overexpressed and previously unexplored in AML, was identified as the target of AYNE. Genetic ablation and pharmacological blockade of VLCAD suppressed FAO metabolism in the AML cell lines TEX 
and OCI-AML2 (AML2). Metabolic assessment of primary patient-derived and normal donorderived mononuclear cells (MNCs) using stable isotope tracers revealed two divergent metabolic fates, resulting in selective AML death and MNC survival. Genetic knockdown or pharmacological inhibition of VLCAD suppressed the engraftment capacity of AML, but not the normal hematopoietic populations, further highlighting the pre-clinical anti-AML efficacy of FAO inhibition at VLCAD. Chapter 6 demonstrated that the odd number of carbon atoms in the aliphatic linear chain, the terminal triple bond, and the $(2 R, 4 R)$-stereochemistry of the hydroxyl groups were critical to AYNE's ability to bind to VLCAD, inhibit FAO, and induce AML death. In summary, this thesis identified VLCAD as a novel anti-AML target and highlighted the pre-clinical efficacy of targeting AML mitochondrial metabolism with AYNE, a selective FAO inhibitor. 


\section{ACKNOWLEDGEMENTS}

My graduate experience over the past six years encompassed the most challenging and rewarding times in my life so far. Overcoming these trials would not have been possible without Paul Spagnuolo, my supervisor, who patiently provided me with constructive criticism, guidance, as well as a supportive research group and network of collaborators who were paramount to the success of this thesis. Thank you, Paul, for helping me grow as a scientist and for supporting my many conference and training excursions. I'd like to thank Profs. Ma and Marangoni, my committee members, for their advice and guidance.

I'd like to thank Andrew and Darin of Quadrilatero group for teaching me high resolution respirometry and how to use a pull up bar. To Arash and Tarek of Nekkar group, thank you for teaching me the basics of HPLC. The success of the liquid scintillation counting, by Prof. Akhtar and Kevin, stereoisomer synthesis, by Profs. O’Doherty and Lewary, Vitor, and Xiaofan, as well as the stable isotope tracing experiments, by Prof. D'Alessandro and Eric, were critical milestones that propelled my research forward. Thank you to Profs. Vockley \& Mohsen, Keaton, Anu, and Clint for welcoming me to Pittsburgh; I experienced kindness, generosity, and a fervent eagerness to collaborate from everyone at your institution. To Dr. Samudio, Profs. Smith, Minden, Hope, Hess, and Schimmer, your technical advice, constructive criticism, and generous material gifts were integral to the publication of my manuscripts and completion of my graduate project. I am extremely grateful for the patience, expertise, and assistance of all our collaborators.

To Preethi and the past members of Spagnuolo group (Leonard, Eric, Sarah, Rowena, Elena), thank you for training me on critical techniques. The research animals involved in my project and I would like to thank Jenn and all CAF staff for their wonderful support. Thank you to Claire, Gary, Profs. Duizer and Mercer - you always made time to chat with me during the good 
and the bad times. To Shane, thank you for the technical support and training - our discussions on the latest games, movies, and shows were always a welcome respite from research.

I am grateful to the exchange students who visited to our lab to share their knowledge, culture and language. To Rita and Federica, fino alla prossima volta! Hasta leugo, Monica, Viri, and Becca. Thank you to Michael and Manal for making the lab a supportive environment! Sarah, thank you for teaching me how to tie complex knots; Varsha, I will always remember chili day; Katrina, our movie nights were always a highlight of my work week; Nil, thank you for teaching me PCR multiple times; Nathan, I wish you the best in your endeavours for medical school. I'd also like to thank Martino for his continued friendship as well as his advice on the technical details of gene editing and the purpose of life beyond graduate school.

This journey began with the trio consisting of Nawaz, Alessia, and I. Nawaz, this project wouldn't have been possible without you. I will remember our late night conversations with taxi drivers in Vancouver, the look on your face after your first bite of Ethiopian cuisine, and your thin green T-shirts. Alessia, thank you for your assistance with countless experiments. You have been a positive impact on me inside and outside the lab. You've never been afraid to point out my faults and errors and I am very grateful to have you as a colleague and friend. I hope to grab pizza with you again at Lucali's someday and that our friendship will continue to flourish after our graduate careers.

Lastly, I would not have been able to accomplish this degree without my mother Linda and my father Peter. Thank you to both of you for your sacrifices and for believing me the many times I told you it's just one more year. Thank you for your unending patience and love through this memorable journey. 


\section{TABLE OF CONTENTS}

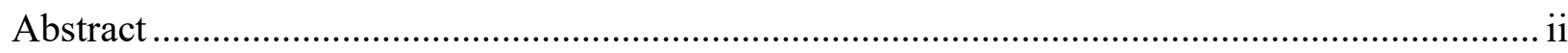

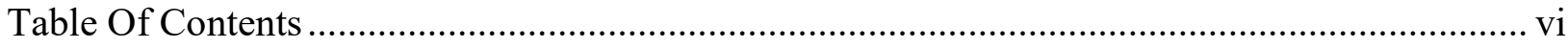

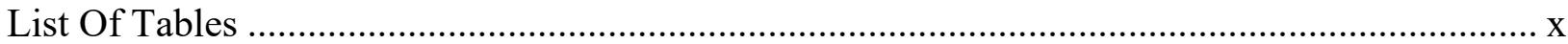

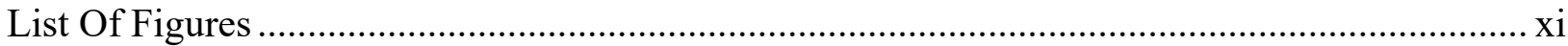

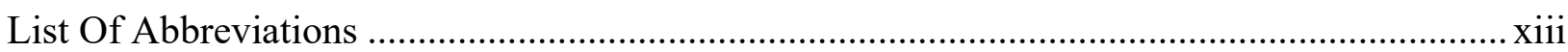

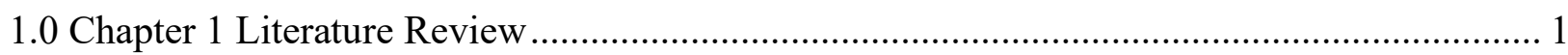

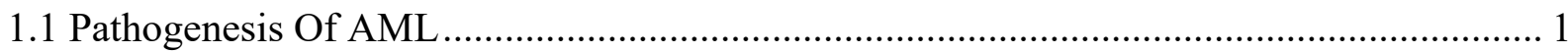

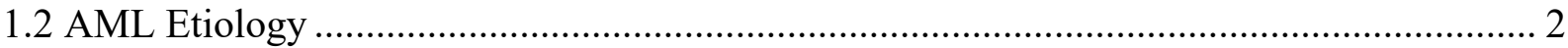

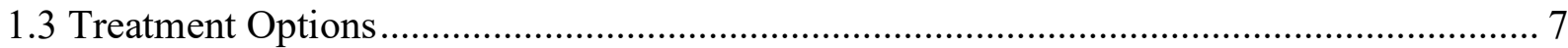

1.3.1 Personalized Therapy Based On Age And Cytogenetics ........................................... 7

1.3.2 Treatment Related Co-Morbidities And Off-Targets Of $7+3$ Therapy .......................... 9

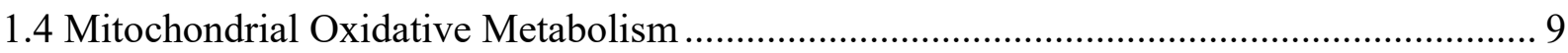

1.4.1 Tricarboxylic Acid (TCA) Cycle ............................................................................... 12

1.4.2 Oxidative Phosphorylation (OXPHOS) At The Electron Transport Chain (ETC) ...... 13

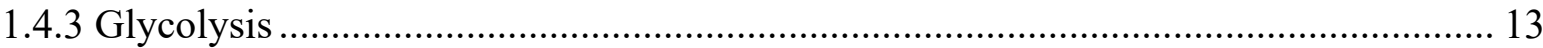

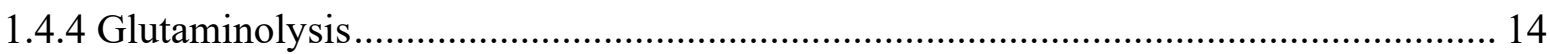

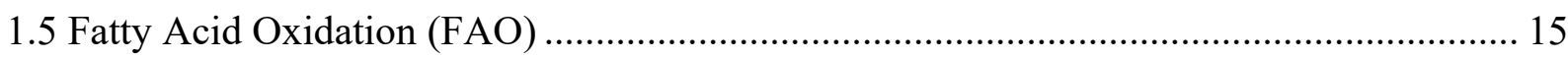

1.5.1 Cytosolic Entry Of Fat From Extracellular Space........................................................ 18

1.5.2 Carnitine Shuttle In The Mitochondrial Membrane ........................................................ 18

1.5.3 $\beta$-Oxidation Cycle Within The Mitochondrial Matrix ………………………………... 19

1.6 Mitochondrial Properties Of AML And Hematopoietic Cells ............................................. 20

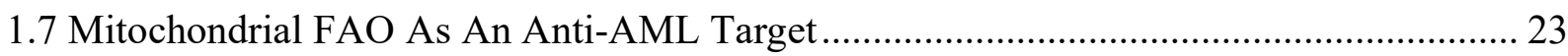

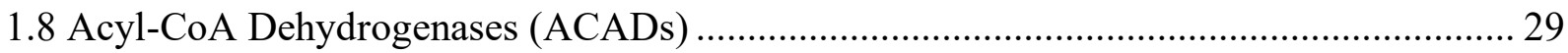

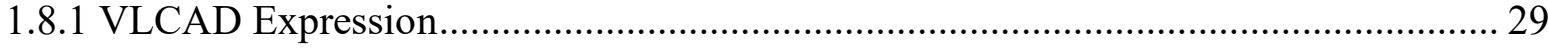

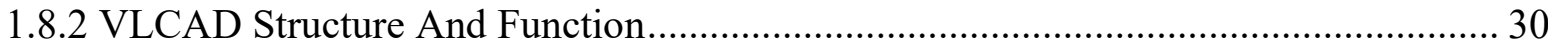

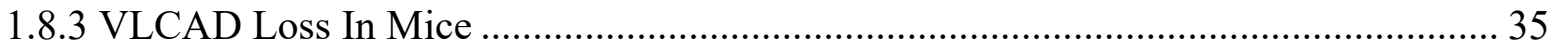

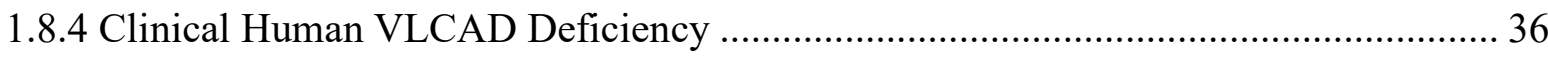

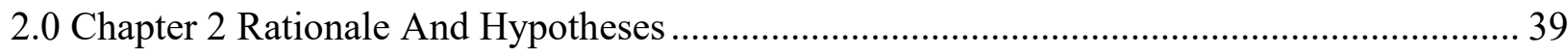

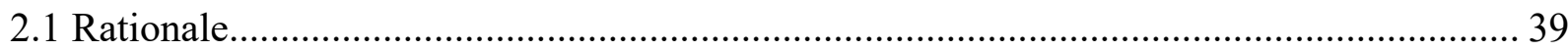

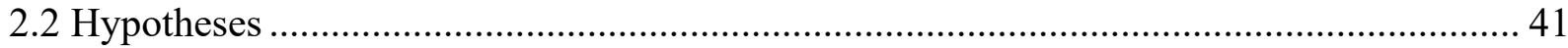

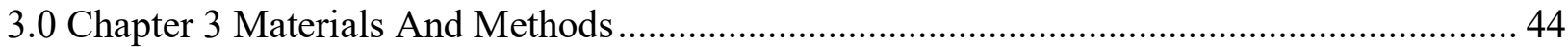

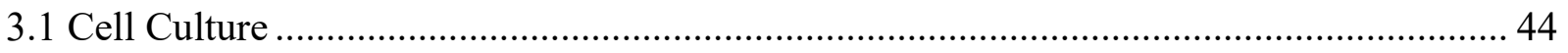


3.2 Source Of Avocatin-B (Avo-B), Avocadyne (AYNE), Avocadene (AENE) ....................... 45

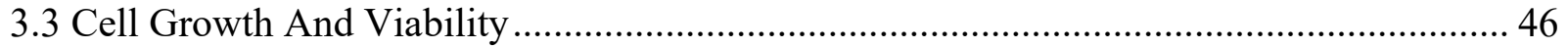

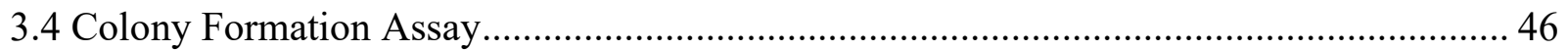

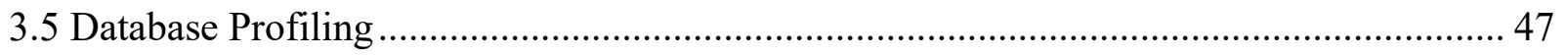

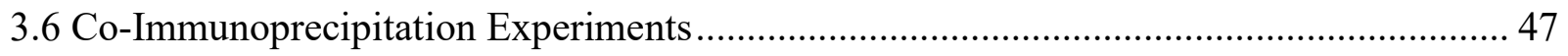

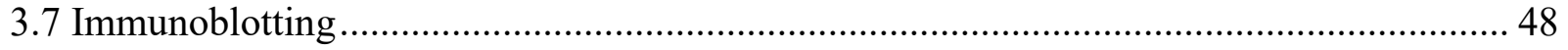

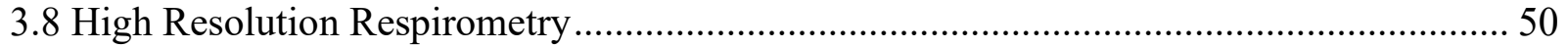

3.9 Fatty Acid Oxidation By Liquid Scintillation Counting ………….................................. 52

3.10 Acyl-CoA Dehydrogenase (ACAD) Activity Assay …………………………............... 52

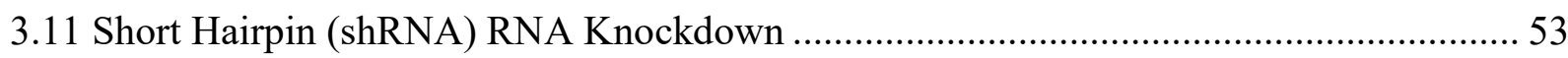

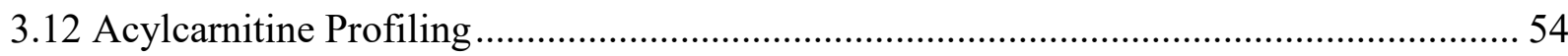

3.13 ATP, PDH (Pyruvate Dehydrogenase) Activity, Mitochondrial Mass Quantification ..... 55

3.14 Ultra High Performance Liquid chromatography (UHPLC) - Mass Spectrometry (MS)

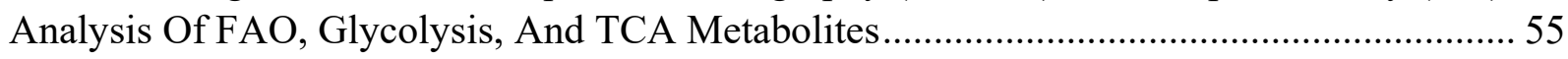

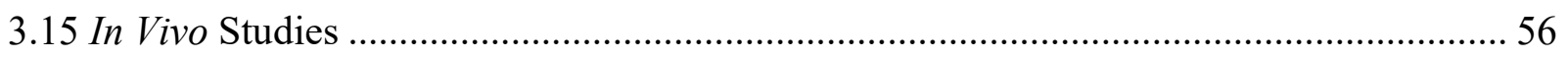

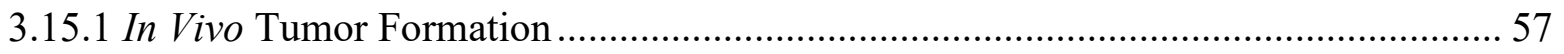

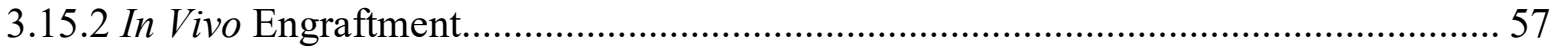

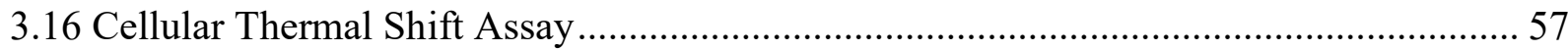

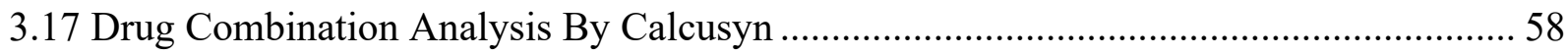

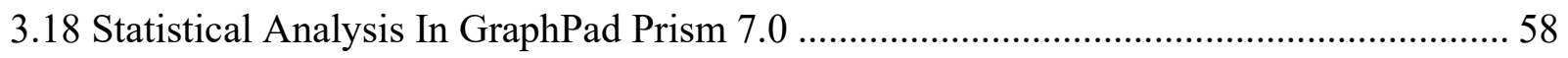

4.0 Chapter 4 Avocadyne (AYNE) Is The Anti-AML Component Of Avo-B, An AvocadoDerived Mixture Of Fatty Alcohols, That Selectively Eliminates AML ....................................... 59

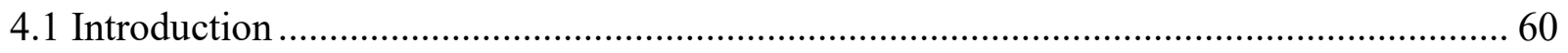

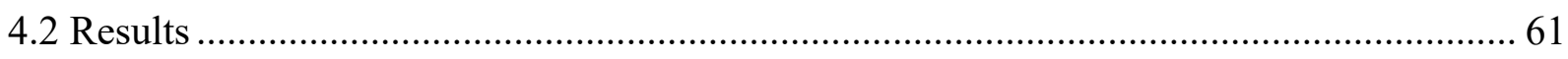

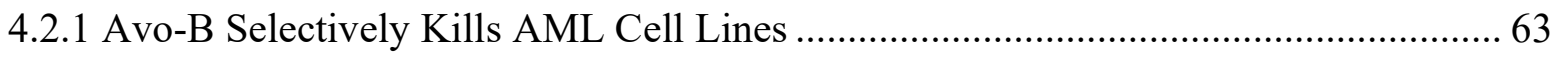

4.2.2 Avo-B Induces Death Of Primary AML Cells, While Sparing Normal MNC Cells ... 65

4.2.3 Avo-B Synergizes With Induction Chemotherapeutics To Induce AML Cytotoxicity 67

4.2.4 The Constituents Of Avo-B Induce AML Death In An Antagonistic Manner ............ 70

4.2.5 AYNE Inhibits Mitochondrial Long Chain FAO To Induce AML Death .................... 74

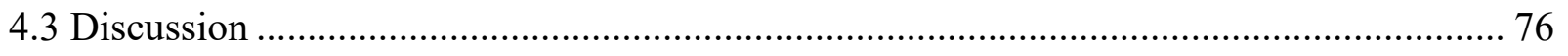

5.0 Chapter 5 The Very Long Chain Acyl-CoA Dehydrogenase (VLCAD) Is The Molecular

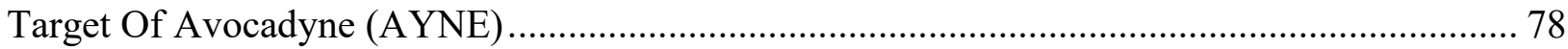

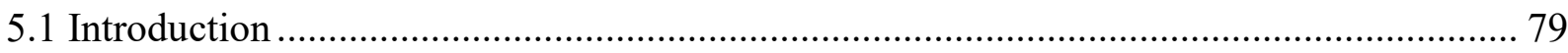


5.2.1 The Gene Acyl-CoA Dehydrogenase Very Long ( $A C A D V L)$ Is Overexpressed In Human AML, Compared To Normal Hematopoiesis . 86

5.2.2 The Very Long Chain Acyl-CoA Dehydrogenase (VLCAD) Protein Is Overexpressed In Human AML, Compared To Normal Hematopoiesis ................................................. 88

5.2.3 VLCAD Knockdown Reduces Leukemia Proliferation And Clonogenic Growth....... 91

5.2.4 VLCAD Knockdown Hinders TEX Engraftment ............................................... 93

5.2.5 VLCAD Knockdown Hinders Intact Leukemia Cell Respiration .............................. 95

5.2.6 VLCAD Knockdown Inhibits C16-Supported ETF Respiration............................... 98

5.2.7 VLCAD Knockdown Alters AML Mitochondrial Metabolism .............................. 101

5.2.8 An Unbiased High Resolution Respirometry (HRR) Based Screen Identifies Avocadyne (AYNE) As A VLCAD Chemical Inhibitor.............................................. 103

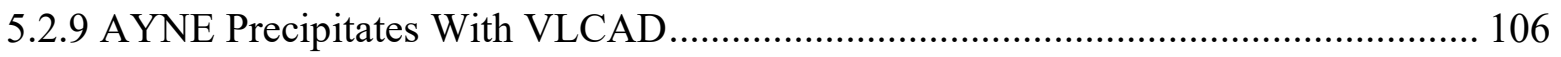

5.2.10 Partial VLCAD Knockdown Does Not Consistently Reduce AML Proliferation ... 109

5.2.11 C16-supported ETF Respiration Is Exclusively Hindered By AYNE, But Not ETO

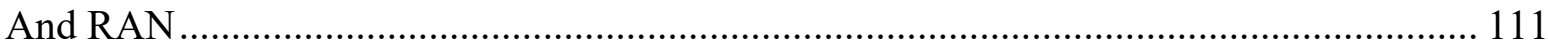

5.2.12 AYNE Kills TEX And AML2 While Suppressing Primary AML Clonogenic Growth

5.2.13 AYNE Directly Inhibits Mitochondrial Long Chain FAO To Hinder Mitochondrial

Respiration In Intact Leukemia Cells ....................................................................... 116

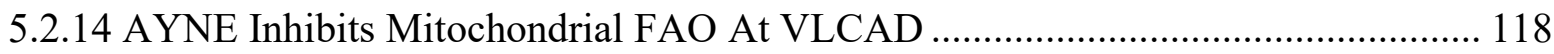

5.2.15 VLCAD Inhibition By AYNE Does Not Alter The Activities Of MCAD Or Complexes I And II Of The ETC ....

5.2.16 AYNE Inhibits Long Chain Mitochondrial FAO In Non-Transformed Fibroblasts 124

5.2.17 Supplementation With The Medium Chain Fat Sodium Heptanoate Rescues AML

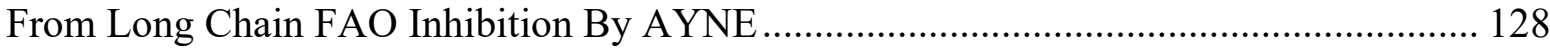

5.2.18 AYNE Triggers ATP Depletion And Selectively Induces Death In CD34+ Primary AML While Sparing CD34+ Normal Hematopoietic Cells ........................................ 131

5.2.19 AYNE Inhibits FAO Activity In TEX And AML2 ......................................... 133

5.2.20 AYNE Inhibits FAO Contribution Into The TCA Cycle In TEX And AML2 ........ 137

5.2.21 AYNE Exclusively Inhibits FAO In Primary AML, While Sparing Normal MNC 140 5.2.22 AYNE Exclusively Inhibits Fat Contribution Into The TCA Cycle In Primary AML, While Sparing Normal MNC 143

5.2.23 VLCAD Inhibition Triggers Compensatory Increase In PDH Activity With No

Significant Alteration In Glycolytic Activity In TEX, AML2, And Primary AML .......... 146 
5.2.24 AYNE Selectively Induces Death Of The AML Population, While Sparing The Normal Blood Cells In Vivo ................................................................................................ 150

5.2.25 AYNE Treatment Is Well Tolerated In NSG Mice ............................................... 153

5.2.26 Isolation Of Primary AML Cells From Mice Treated AYNE ............................... 155

5.2.27 AYNE Binds To And Inhibits VLCAD In Vivo ................................................... 158

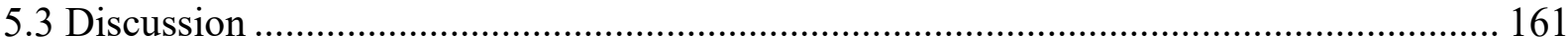

6.0 Chapter 6 A Structure-Activity Study Of Avocadyne (AYNE)'s Anti-AML Mechanism Of

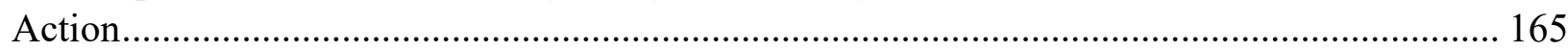

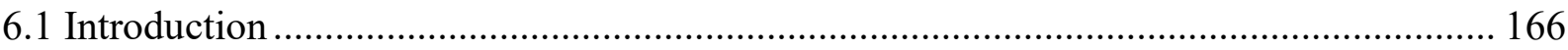

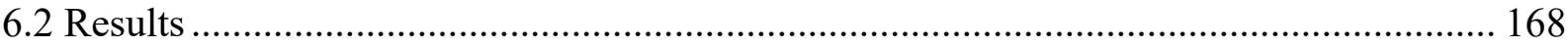

6.2.1 AYNE's Terminal Triple Bond And An Odd Number Of Carbon Atoms Are Critical

To Its AML Cytotoxicity ....................................................................................... 170

6.2.2 AYNE's Terminal Triple Bond And Odd Numbered Carbon Chain Are Critical To Its Ability To Suppress Mitochondrial Respiration..................................................... 172

6.2.3 Stereochemistry At AYNE’s Chiral Carbons Are Critical To Its AML Cytotoxicity

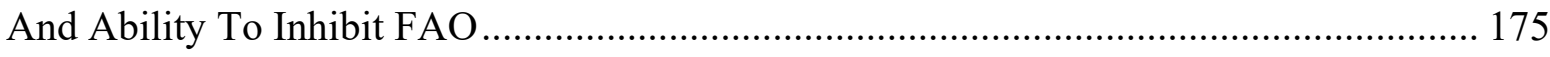

6.2.4 Stereochemistry At Avocadene's Chiral Carbons Are Critical To AML Cytotoxicity And Ability To Inhibit Mitochondrial Respiration...................................................... 178

6.2.5 (R)-Stereochemistry Is Critical For AYNE To Directly Interact With VLCAD....... 180

6.2.6 $(2 R, 4 R)$-Stereochemistry Is Critical For AYNE's In Vivo Anti-AML Activity......... 183

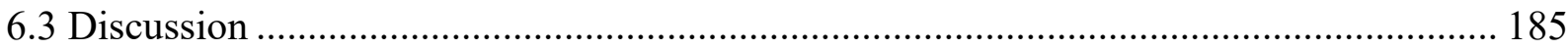

7.0 Chapter 7 Discussion, Limitations, And Conclusion................................................... 189

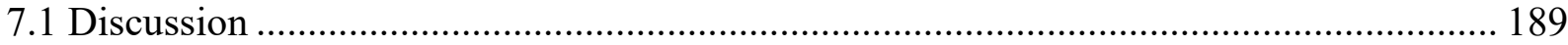

7.2 Limitations And Future Directions ......................................................................... 193

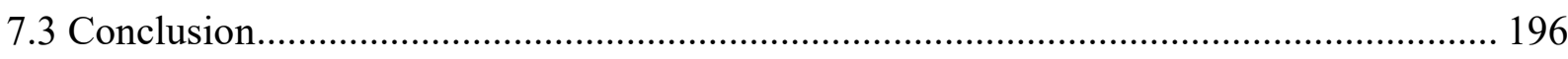

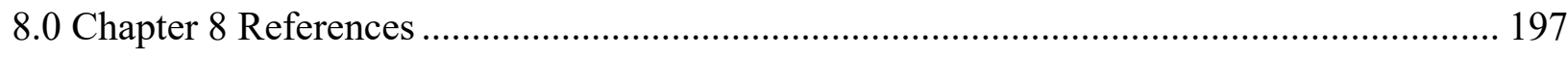

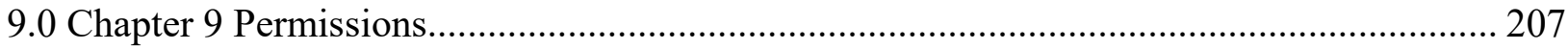

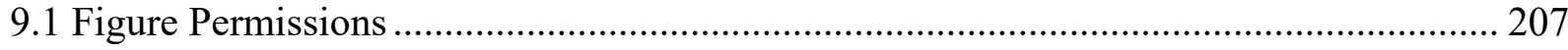

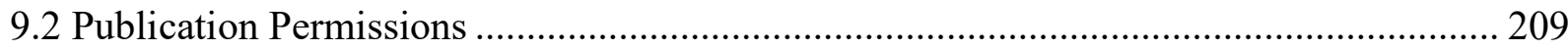

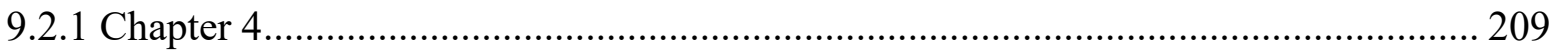

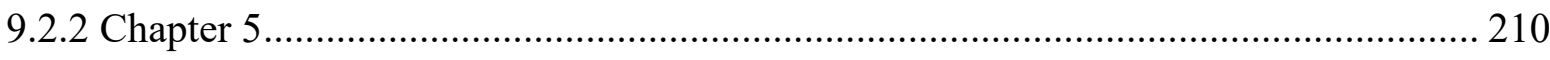

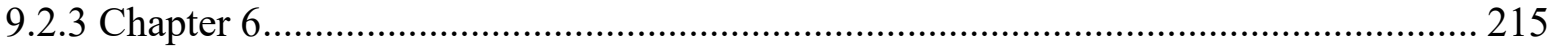




\section{LIST OF TABLES}

Table 1.5.1 List of protein names, short form aliases, and the encoding genes for the

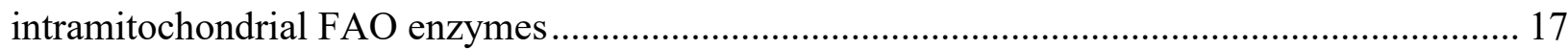

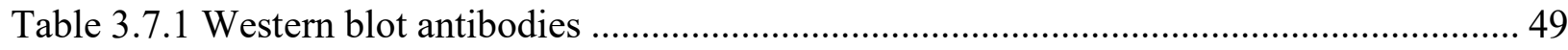

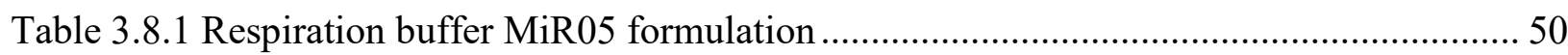

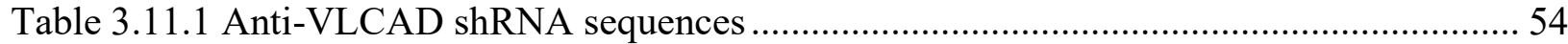

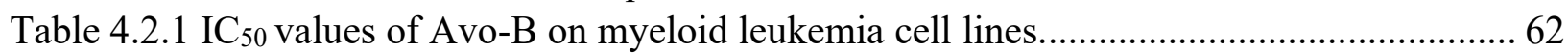

Table 4.2.2 Combination index values with combinations of avo-B and induction

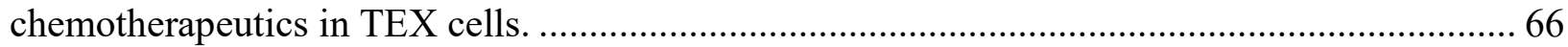

Table 4.2.3 Summary of $\mathrm{IC}_{50}$ values of the avo-B mixture and its constituents AYNE and AENE on TEX and AML2 presented in figure 3.2.4 A, B.

Table 4.2.4 Summary of combination index values for the avo-B mixture on TEX and AML2

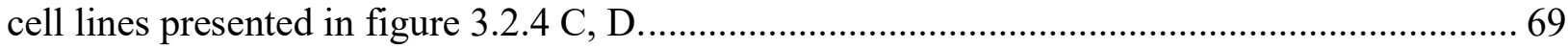

Table 4.2.5 Cytogenetics for patient IDs for Chapter 4 ..................................................... 75

Table 5.2.1 Comparison of intramitochondrial FAO gene expression in GSE9476 ................. 82

Table 5.2.2 Comparison of intramitochondrial FAO gene expression in GSE13204 ................ 83

Table 5.2.3 Comparison of intramitochondrial FAO gene expression in GSE1 159 ................. 84

Table 5.2.4 Comparison of ACADVL gene expression between AML and normal samples in

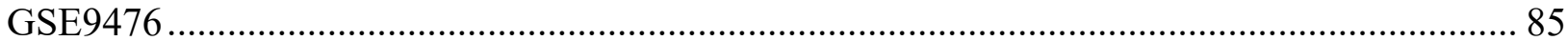

Table 5.2.5 Comparison of ACADVL gene expression between AML and normal samples in

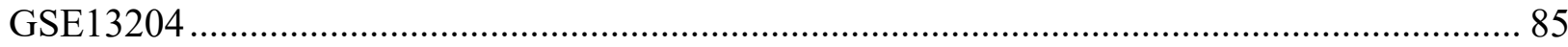

Table 5.2.6 Comparison of ACADVL gene expression between AML and normal samples in

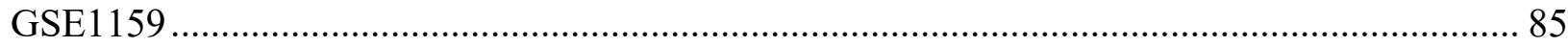

Table 5.2.7 Comparing VLCAD expression in primary AML and MNC to TEX ..................... 87

Table 5.2.8 Quantification of AYNE in VLCAD-enriched fractions ...................................... 105

Table 5.2.9 Summary of statistics for data presented in figure 5.2.19 and 5.2.20 ................. 136

Table 5.2.10 Summary of statistics for data presented in Figure 5.2.21 ................................. 142

Table 5.2.11 Summary of statistics for data presented in Figure 5.2.21 and 5.2.22 ............... 142

Table 5.2.12 Summary of statistics for data presented in Figure 5.2.23 ................................. 145

Table 5.2.13 Summary of statistics for Figure 4.2.25 .................................................. 152

Table 5.2.14 Cytogenetics of patient IDs used in chapter 5 ................................................ 159

Table 6.2.1 IC $\mathrm{IC}_{50}$ values for AYNE 1, HATA, PATA in TEX and AML2 ............................. 169

Table 6.2.2 AYNE recovered from AML cells treated with compounds 1-4 by co-IP ............. 179

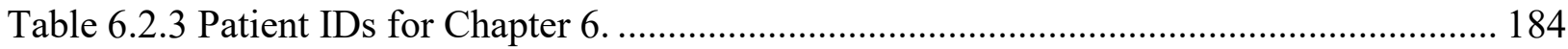




\section{LIST OF FIGURES}

Figure 1.2.1 Hematopoiesis and leukemogenesis are clonal hierarchies...................................... 4 Figure 1.2.2 The NSG engraftment assay identifies the hematopoietic and leukemia cell of origin

Figure 1.4.1 Oxidation of glucose, glutamine, and fat fuel mitochondrial oxidative metabolism 11

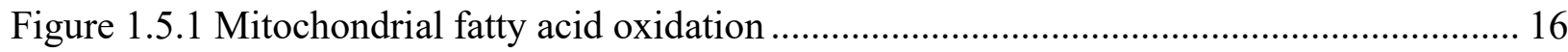

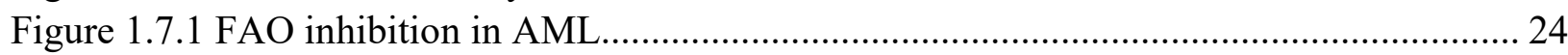
Figure 1.8.1 ACADs catalyze the oxidation of a fat by introducing an unsaturation across the C2C3 bond... 31

Figure 1.8.2 A catalytic glutamic acid residue initiates the ACAD reaction in the fat binding

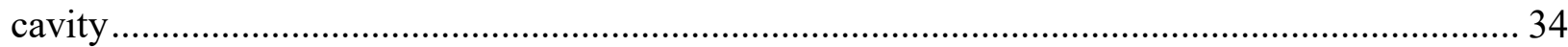

Figure 2.1.1 Structure of the constituents of avocatin-B (avo-B) …………………………....... 40

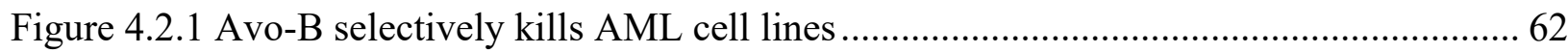

Figure 4.2.2 Avo-B induces death of primary AML cells, while sparing normal MNC cells...... 64 Figure 4.2.3 Avo-B synergizes with induction chemotherapeutics Ara-C and DOX to kill TEX.

Figure 4.2.4 The constituents of avo-B induce AML death in an antagonistic manner ................6 68

Figure 4.2.5 AYNE inhibits mitochondrial long chain FAO in AML....................................... 72

Figure 5.2.1 The gene acyl-CoA dehydrogenase very long (ACADVL) is overexpressed in human AML, compared to normal hematopoiesis.

Figure 5.2.2 The very long chain acyl-CoA dehydrogenase (VLCAD) protein is overexpressed in

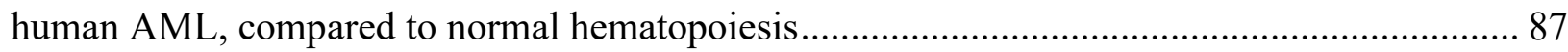

Figure 5.2.3 VLCAD knockdown reduces leukemia proliferation and clonogenic growth ......... 90

Figure 5.2.4 VLCAD knockdown hinders TEX engraftment..................................................... 92

Figure 5.2.5 VLCAD knockdown hinders intact leukemia cell respiration .................................. 94

Figure 5.2.6 VLCAD knockdown inhibits C16-supported ETF respiration.................................. 97

Figure 5.2.7 VLCAD knockdown alters AML mitochondrial metabolism................................... 99

Figure 5.2.8 An unbiased HRR-based screen identifies avocadyne (AYNE) as a VLCAD

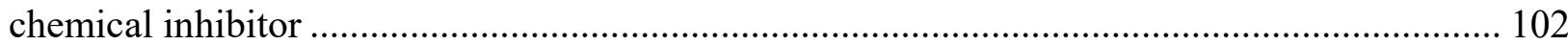

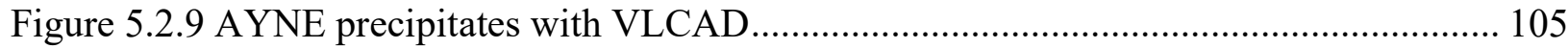

Figure 5.2.10 Partial VLCAD knockdown did not significantly reduce AML proliferation to the same extent as a complete VLCAD knockdown .................................................................. 108

Figure 5.2.11 C16-supported ETF respiration is exclusively hindered by AYNE, but not ETO

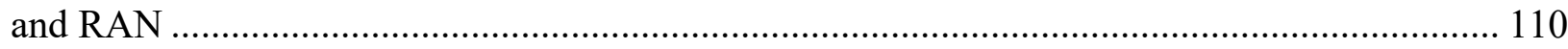

Figure 5.2.12 AYNE kills TEX and AML2 while suppressing primary patient-derived AML

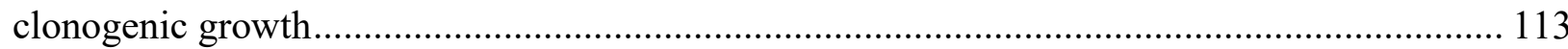
Figure 5.2.13 AYNE directly inhibits mitochondrial long chain FAO to hinder mitochondrial

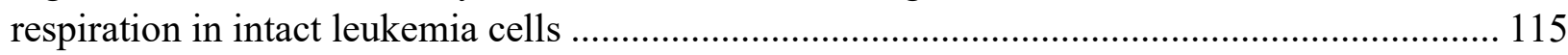

Figure 5.2.14 AYNE inhibits mitochondrial FAO at VLCAD ................................................ 117 Figure 5.2.15 VLCAD inhibition by AYNE does not alter the activities of MCAD or complexes I

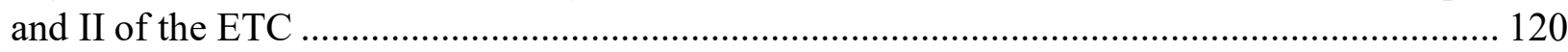
Figure 5.2.16 AYNE inhibits long chain mitochondrial FAO in non-transformed fibroblasts .. 122 
Figure 5.2.17 Supplementation with the medium chain fat sodium heptanoate rescues AML from

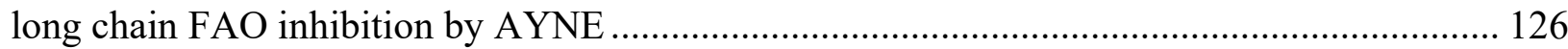
Figure 5.2.18 AYNE triggers ATP depletion to selectively induce death in CD34+ primary AML while sparing CD34+ normal hematopoietic cells............................................................ 130

Figure 5.2.19 AYNE inhibits FAO activity in TEX and AML2 ....................................... 132

Figure 5.2.20 AYNE inhibits FAO contribution into the TCA cycle in TEX and AML2 ........ 135

Figure 5.2.21 AYNE exclusively inhibits FAO in primary AML, while sparing normal MNC 139 Figure 5.2.22 AYNE exclusively inhibits fat contribution into the TCA cycle in primary AML,

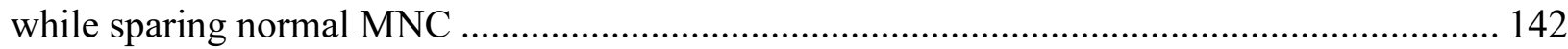

Figure 5.2.23 VLCAD inhibition triggers compensatory increase in PDH activity that is insufficient to alter overall glycolytic activity in TEX, AML2, and primary AML 145 Figure 5.2.24 AYNE selectively induces death of the AML population, while sparing the normal

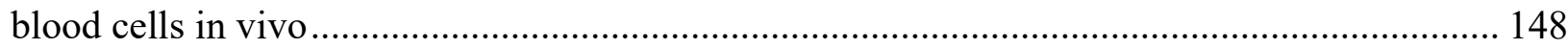

Figure 5.2.25 AYNE treatment is well tolerated by NSG mice.......................................... 152

Figure 5.2.26 Isolation of primary AML cells from mice treated AYNE .............................. 154

Figure 5.2.27 AYNE binds to and inhibits VLCAD in vivo ............................................ 157

Figure 6.2.1 AYNE's terminal triple bond and an odd number of carbon atoms are critical to its

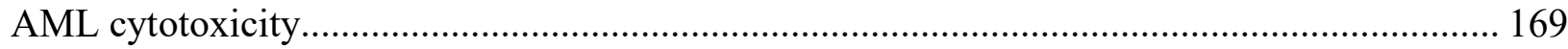

Figure 6.2.2 AYNE's terminal triple bond and an odd number of carbon atoms in its aliphatic linear chain are critical to its ability to suppress mitochondrial respiration. .......................... 171 Figure 6.2.3 Stereochemistry at AYNE's chiral carbons are critical to its AML cytotoxicity and

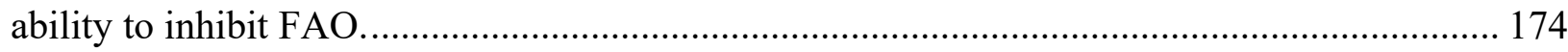
Figure 6.2.4 Stereochemistry at avocadene's chiral carbons are critical to AML cytotoxicity and ability to inhibit mitochondrial respiration. ................................................................ 177 Figure 6.2.5 (R)-stereochemistry is critical for AYNE to directly interact with VLCAD. ....... 179 Figure 6.2.6 (2R,4R)-stereochemistry is critical for AYNE's in vivo anti-AML activity......... 182 


\section{LIST OF ABBREVIATIONS}

(c)DNA (Complementary) deoxyribonucleic acid

7AAD 7-Aminoactinomycin D

$\AA$

Angstrom $\left(1 * 10^{-10}\right.$ meters)

$\mathrm{AC}$

Acyl-carnitine

ACAD

Acyl-CoA dehydrogenase

$A C A D V L$

Acyl-CoA dehydrogenase very long (gene)

$\mathrm{ACC} 2$

Acetyl-CoA carboxylase 2

ADP or ATP

Adenosine di or triphosphate

AENE

Avocadene

AML

AntiA

Acute myeloid leukemia

APOC2

Antimycin A

Ara-C

Apolipoprotein C-2

ATF4

Avo-B

AYNE

Bcl-2

Cytarabine

Activating transcription factor 4

Avocatin-B

Avocadyne

BM

B cell lymphoma 2

BMS-0309403

Bone marrow

\section{BSA}

2-[[2'-(5-Ethyl-3,4-diphenyl-1H-pyrazol-1-yl)[1,1'-biphenyl]-3-yl]oxy]acetic acid

C16-CoA

Bovine serum albumin

C7

Palmitoyl-CoA

C8-CoA

Sodium heptanoate

Carn

Octanoyl-CoA

CD

L-carnitine

CI

Cluster of differentiation

CoA

Combination index

Co-IP

Coenzyme A

$\mathrm{cVLCAD}^{-/-}$

Co-immunoprecipitation

DIO

DMSO

Cardiac specific VLCAD knockout

DOX

Diet induced obesity

EDTA

Dimethyl sulfoxide

ETC

Doxorubicin

ETF

Ethylenediaminetetraacetic acid

Electron transport chain

ETFDH

Electron transfer flavoprotein

ETO

ETF dehydrogenase

FABP4

Etomoxir

$\mathrm{FADH}_{2}$

Fatty acid binding protein 4

FAO

Flavin adenine dinucleotide (reduced)

FBS

Fatty acid oxidation

Fetal bovine serum

FCCP

Carbonyl cyanide 4-(trifluoromethoxy)phenylhydrazone

FCS

Fetal calf serum

FDA

US Food and Drug Administration 


$\begin{array}{ll}\text { FLT3 } & \text { Fms related tyrosine kinase 3 } \\ \text { GAPDH } & \text { Glyceraldehyde 3-phosphate dehydrogenase } \\ \text { GSE } & \text { Genomic spatial event } \\ \text { GSH } & \text { Glutathione } \\ \text { HRR } & \text { High resolution respirometry } \\ \text { HSC } & \text { Hematopoietic stem cell } \\ \text { IDH } & \text { Isocitrate dehydrogenase } \\ \text { IL } & \text { Interleukin } \\ \text { IMDM } & \text { Iscove's modified dulbecco medium } \\ \text { IMM } & \text { Inner mitochondrial membrane } \\ \text { IMS } & \text { Intermitochondrial space } \\ \text { ISR } & \text { Integrated stress response } \\ \text { ISRIB } & \text { Integrated stress response inhibitor } \\ \text { LCAD } & \text { Long chain acyl-CoA dehydrogenase } \\ \text { LSC } & \text { Leukemia stem cell } \\ \text { MAL } & \text { Malate } \\ \text { MALON } & \text { Malonate } \\ \text { MCAD } & \text { Medium chain acyl-CoA dehydrogenase } \\ \text { MEM } & \text { Minimum essential medium eagle } \\ \text { MiR05 } & \text { Mitochondrial respiration buffer 05 } \\ \text { MNC } & \text { Mononuclear cell } \\ \text { MSG } & \text { Monosodium glutamate } \\ \text { MTS } & \text { [3-(4,5-dimethylthiazol-2-yl)-5-(3-carboxymethoxyphenyl)-2-(4- } \\ & \text { sulfophenyl)-2H-tetrazolium, inner salt } \\ \text { NAD(P)H } & \text { Nicotinamide adenine (phosphate) dinucleotide (reduced) } \\ \text { NK } & \text { Natural killer } \\ \text { NPM1 } & \text { Nucleophosmin 1 } \\ \text { NSG } & \text { Non-obese severe combined immunodeficient gamma } \\ \text { OC } & \text { Octanoyl-carnitine } \\ \text { OCI-AML2 } & \text { Ontario Cancer Institute - Acute myeloid leukemia 2 } \\ \text { OE } & \text { Overexpression } \\ \text { Oli } & \text { Oligomycin } \\ \text { OMM } & \text { Outer mitochondrial membrane } \\ \text { OXPHOS } & \text { Oxidative phosphorylation } \\ \text { PA-BSA } & \text { Palmitate coupled to BSA } \\ \text { PBS } & \text { Phosphate buffered saline } \\ \text { PC } & \text { Palmitoyl-carnitine } \\ \text { PDH } & \text { Pyruvate dehydrogenase } \\ \text { PHD3 } & \text { Prolyl hydroxyl domain protein 3 } \\ \text { PYR } & \text { Sodium pyruvate } \\ \text { RAN } & \text { Ranolazine } \\ \text { RIPA } & \text { Radio-Immunoprecipitation Assay } \\ \text { ROT } & \text { Rotenone } \\ \text { SCAD } & \text { Short chain acyl-CoA dehydrogenase } \\ \text { SCF } & \text { Stem cell factor } \\ \text { SDS-PAGE } & \text { sodium dodecyl sulphate-polyacrylamide gel electrophoresis } \\ & \text { a }\end{array}$


shRNA Short hairpin ribonucleic acid

SLC Solute carrier family

SSO Sulfo-N-succinimidyl oleate

TCA Tricarboxylic acid

TEX TLS (FUS RNA binding protein)-ERG (ETS transcription factor) Immortalized

TMZ Trimetazidine

TNF-alpha Tumor necrosis factor alpha

UHPLC-MS Ultra high performance liquid chromatography coupled to mass spectrometry

UQ

VLCAD Very long chain acyl-CoA dehydrogenase 


\subsection{Chapter 1 Literature Review}

\subsection{Pathogenesis Of AML}

Acute myeloid leukemia (AML) is a deadly hematological malignancy, resulting in the uncontrolled proliferation and accumulation of leukemia blasts that fail to fully differentiate into functional myeloid cells. It initiates in the bone marrow and manifests in peripheral blood. AML severely hinders normal hematopoiesis, resulting in the marked depletion of functional mature myeloid cells, such as erythrocytes, monocytes, neutrophils, eosinophils, basophils, platelets and the accumulation of malignant leukemia blasts (Löwenberg et al., 2011). Symptoms caused by the loss of functional mature myeloid cells include shortness of breath, chronic fatigue, increased vulnerability to bacterial and fungal infections, and increased susceptibility to bruising (Shipley \& Butera, 2009).

While AML is the most common acute leukemia and occurs at all ages, the rate of incidence increases with age with a median age of diagnosis at 72 . The age adjusted rate of AML incidence among the adult population ( $>18$ years) is $30-40$ per million while the pediatric population $(0-14$ years) is 7.7 per million (Khwaja et al., 2016). Initial treatment will attempt to achieve remission, a state in which the majority of leukemia blasts are eliminated and normal blood development resumes (Löwenberg et al., 2011); however, no current chemotherapeutic is curative (Löwenberg et al., 2011). Relapse, a return of the disease after initial treatment, is the primary cause of AML patient death. For patients under 60 , the remission rate is approximately $70 \%$; relapse occurs producing a survival rate of 50\%. Most mature patients (above 65) relapse, with a five year survival rate of less than $10 \%$ (Khwaja et al., 2016). The abysmal survival rates of the mature population are primarily due to AML relapse and a reduced tolerance to harsh, extended treatment regimens (Löwenberg et al., 2011). 


\subsection{AML Etiology}

AML develops after hematological progenitors acquire successive, non-random genetic abnormalities that impair differentiation and dysregulate proliferation rates (Reinisch et al., 2015)(Horton \& Huntly, 2012). Multiple progenitors may undergo transformation resulting in a highly heterogeneous, yet clonal malignancy (Reinisch et al., 2015). Historically, under the French-American-British classification system, AML subtypes were classified, based on cell morphological changes, into groups M0 through to M7 (Löwenberg et al., 2011). Currently, patients are now stratified based on risk level (low, intermediate, adverse), depending on the presence of specific chromosomal or genetic level aberrations as well as clinical features (age, response to previous therapy, de novo or relapse AML) (Khwaja et al., 2016). Single mutations are rarely sufficient to initiate transformation; AML typically develops due to the successive acquisition of driver mutations, which are mutations that promote a leukemia phenotype characterized by impaired differentiation and increased proliferation. Some mutations cooperate to promote a leukemia phenotype; for example, the trio of DNA (cytosine-5)-methyltransferase 3A (DNMT3A), nucleophosmin 1 (NPM1), and Fms related tyrosine kinase 3 (FLT3) and the duo of Tet methylcytosine dioxygenase 2 (TET2) and isocitrate dehydrogenase 1/2 (IDH1/2) are frequently observed concurrently (Horton \& Huntly, 2012). While many mutations contributing to AML pathology have been identified, about $55 \%$ of patients are cytogenetically normal with no detectable chromosomal or genetic abnormalities (Reinisch et al., 2015).

Normal hematopoiesis is organized as a hierarchy with the hematopoietic stem cells (HSCs), a rare stem cell population with high self renewal capacity and multi-lineage potential, at the apex (Figure 1.2.1 A)(Khwaja et al., 2016). Self renewing HSCs undergo asymmetric division, producing one daughter cell and one cell identical to the parent - this maintains the rare stem cell 
population, ensuring a constant supply of blood cells for a human's entire lifespan. Multi-lineage potential is the potential to differentiate into more specialized myeloid progenitor cells that will eventually terminally differentiate into a functional myeloid cell such as: thrombocytes, monocytes, natural killer (NK) cells, erythrocytes, eosinophils, basophils (Horton \& Huntly, 2012).

The AML population is similarly organized into a hierarchy with the leukemia stem cells (LSCs), analogous to the hematopoietic stem cell population, at the apex of the malignant population (Figure 1.2.1 B). The bulk AML population are leukemia blasts that will never fully differentiate into mature, functional myeloid cells. Compared to the blast population, LSCs have increased self renewal capabilities and a distinct metabolism that contributes to chemoresistance and relapse initiation. 

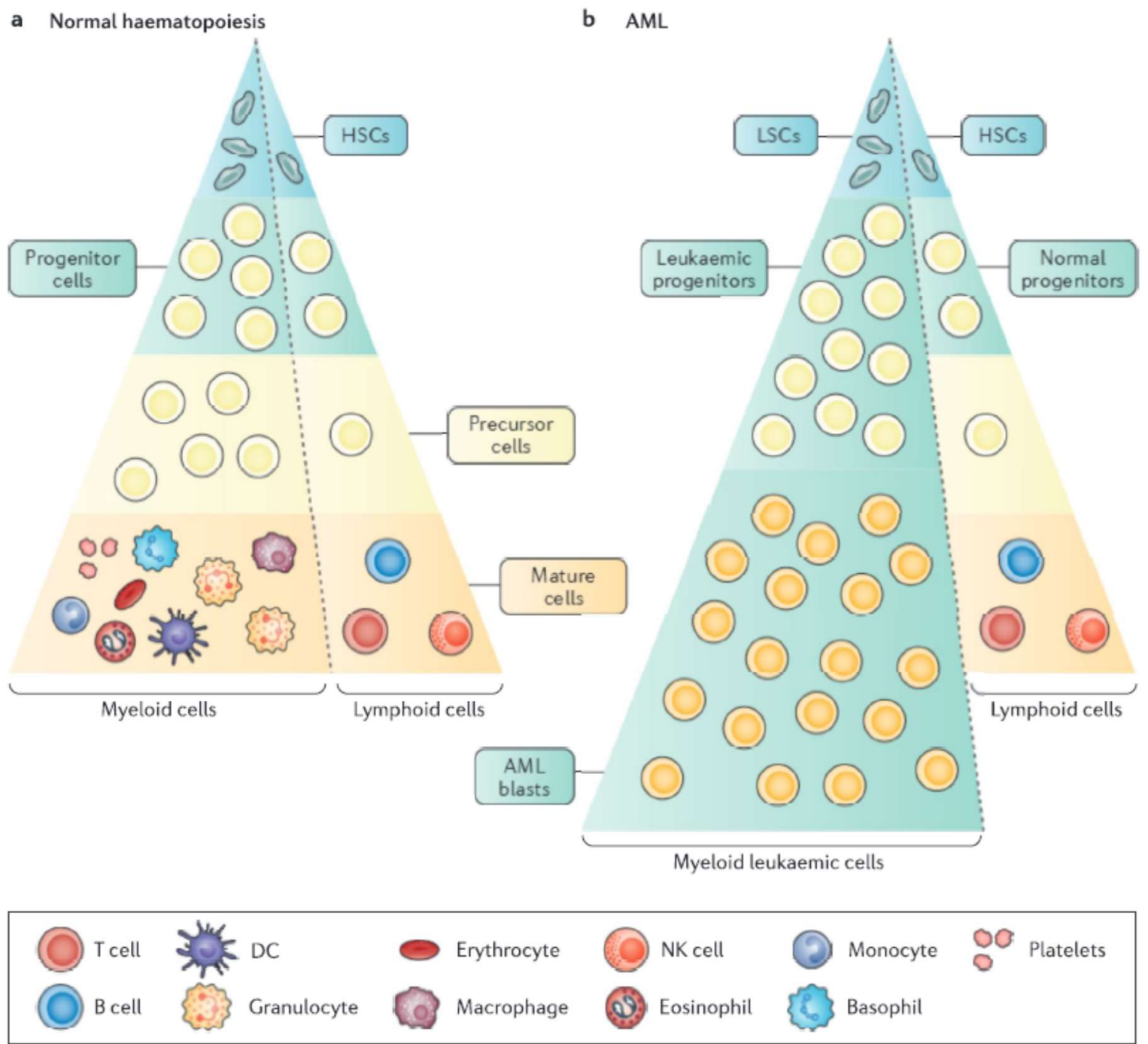

\section{Figure 1.2.1 Hematopoiesis And Leukemogenesis Are Clonal Hierarchies}

(A) The normal hematopoietic population is organized into a clonal hierarchy consisting of the HSCs, progenitor cells, precursors cells, and mature myeloid cells where the HSCs are the least differentiated and the mature myeloid cells are the most differentiated. HSCs exhibit high selfrenewal capacity, give rise to progenitor cells, and can differentiate into all myeloid lineages. As progenitor cells mature, they gradually lose proliferative capacity and eventually commit to a single myeloid lineage, eventually differentiating into a mature myeloid cell. (B) The neoplastic myeloid leukemia population is organized into a clonal hierarchy consisting of LSCs and the bulk leukemia blast cells. LSCs exhibit high self-renewal capacity and give rise to the myeloid blast cells, which comprise the bulk of the leukemia populations. Abbreviations: HSC: hematopoietic stem cell; LSC: leukemia stem cell; DC: dendritic cell; NK: natural killer. Reprinted with permission (Khwaja et al., 2016). 
Depending on cell lineage and differentiation status, cells will display various cluster of differentiation (CD) cell-surface proteins, allowing the identification and isolation of specific subpopulations. A hallmark feature of the HSC and LSC populations is the ability to engraft in the bone marrow of a non-obese severe combined immunodeficient gamma (NSG) mouse and recapitulate their entire respective populations (Figure 1.2.2). This engraftment assay identified the Lin-/CD34+/CD38- subpopulation as the AML initiating population. This subpopulation engrafted into the femoral bone marrow of a NSG mouse and re-capitulated the entire AML population. The subpopulation was subsequently recovered and injected into a secondary set of NSG mice. These LSCs engrafted and re-capitulated the entire AML population, demonstrating a capacity for selfrenewal in successive engraftments (Figure 1.2.2). The HSC subpopulation was similarly identified with the engraftment assay as a population of primitive normal cells that could recapitulate the entire hematopoietic population in a NSG mouse (Figure 1.2.2) (Bonnet \& Dick, 1997)(Hope et al., 2004). Given AML heterogeneity, recent studies have since identified leukemia initiating cells in populations with different CD profiles including, for example, the Lin+/CD38+ and Lin+/CD45RA+ subpopulations. Certain AML populations exhibited the same CD protein profile as normal hematopoietic progenitors, underscoring the need for selective chemotherapies (Chao et al., 2008). Taken together, the NSG engraftment assay is a well-established experiment allowing in vivo assessment of AML or hematopoietic populations (Bonnet \& Dick, 1997)(Hope et al., 2004)(Chao et al., 2008). Once AML or hematopoietic cells have engrafted in NSG mice, the in vivo efficacy of novel therapeutics can also be assessed. 


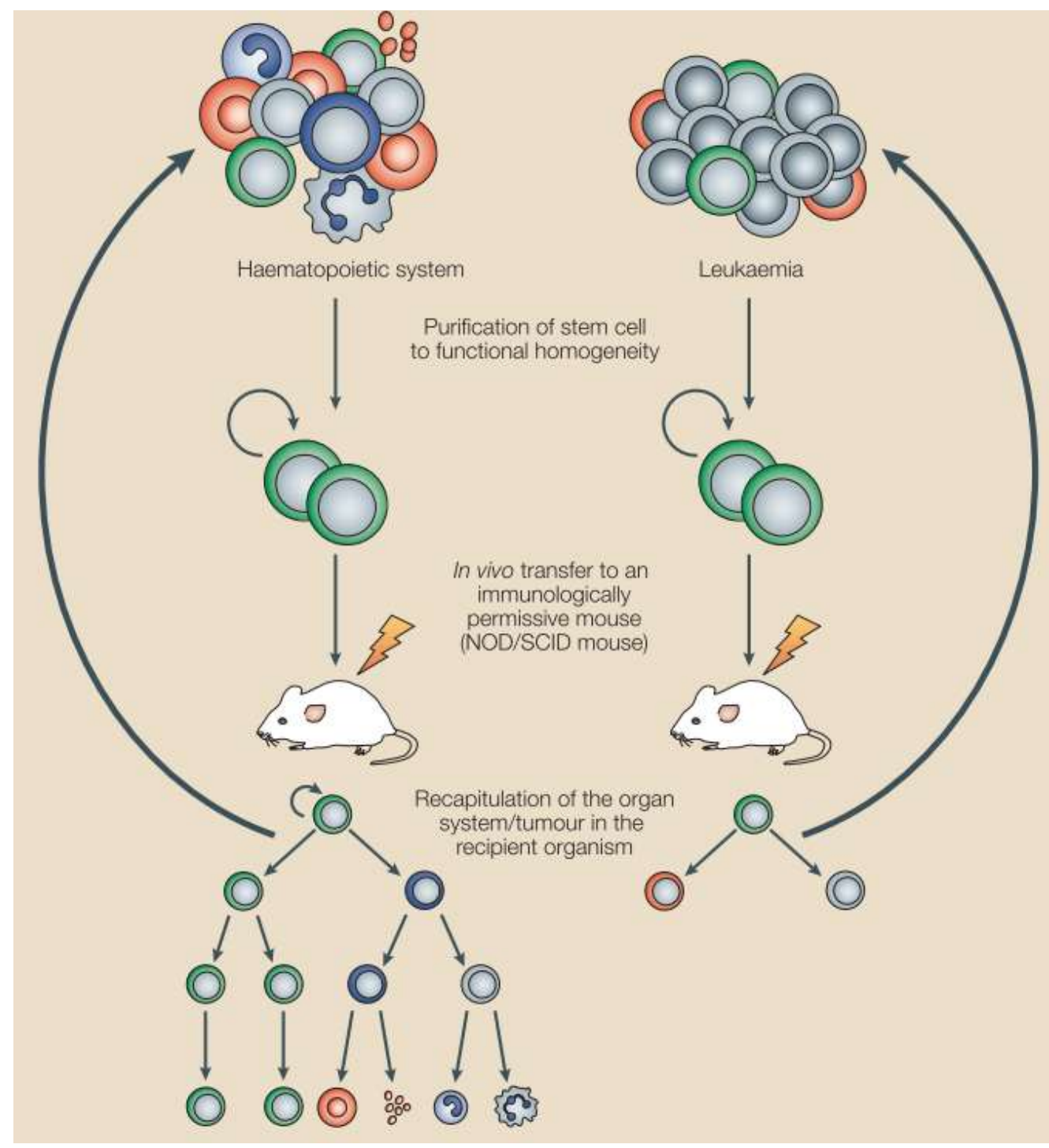

\section{Figure 1.2.2 The NSG Engraftment Assay Identifies The Hematopoietic And Leukemia Cell Of Origin}

The above schematic outlines the engraftment of hematopoietic and leukemia stem cells into immunodeficient mice. By definition, a stem cell must demonstrate self-renew capacity and the ability to reconstitute an entire cell lineage over long periods of time. The cell of interest is transferred to an immune-deficient recipient, allowed to engraft to the bone marrow, and demonstrated self-renewal and differentiation. (Top of figure) HSC and LSC populations are first isolated from bulk hematopoietic and leukemia populations. (Middle of figure) HSC and LSC populations were identified by engrafting into NSG mice, a strain lacking B, T, NK cells with defective macrophage activity, and reconstituting the entire hematopoietic (left side) and leukemia (right side) populations. (Bottom of figure) Assessment of cell populations accomplished with flow cytometry following antibody staining for cell surface markers. Abbreviations: NOD/SCID: non-obese diabetic severe combined immunodeficiency. Reprinted with permission (Huntly \& Gilliland, 2005). 


\subsection{Treatment Options}

Administered intravenously, the $7+3$ regimen has been the primary combination therapy used to induce remission to most AML patients (Shipley \& Butera, 2009). The treatment cycle consists of seven days of continuous infusion of the nucleoside analog cytarabine (Ara-C) at 100$200 \mathrm{mg} / \mathrm{m}^{2}$ and three days of the anthracycline daunorubicin at $60 \mathrm{mg} / \mathrm{m}^{2}$ (Lichtman, 2013). The goal is to induce remission where the patient's bone marrow and peripheral blood have $<5 \%$ blast population and normal hematopoiesis has been restored. Depending on the patient's risk level, consolidation and maintenance regimens consisting of ara-C or anthracycline dose escalation or allogeneic hematopoietic stem cell transplantation, a high-risk procedure, will attempt to reduce the possibility of relapse (Shipley \& Butera, 2009).

\subsubsection{Personalized Therapy Based On Age And Cytogenetics}

Therapeutic options have remained virtually unchanged in the past four decades with most AML patients undergoing either $7+3$ or a variation of the regimen with higher or lower doses of additional or fewer cycles. The inclusion of a third chemotherapeutic to augment existing regimens will be determined based on individual patient cytogenetic and clinical features (Lichtman, 2013).

Despite the extensive profiling of genetic abnormalities, therapeutic options in AML remain limited with the majority of patients still receiving $7+3$ therapy due to its success in inducing initial remission and despite the inevitability of relapse. In 2018, the U.S. Food and Drug Association (FDA) approved CPX351, a liposomal formulation of cytarabine and daunorubicin

optimized for controlled release, minimal systemic circulation, and preferential uptake by leukemia cells in the bone marrow. A study with patients aged $60-75$ observed a $47 \%$ remission rate vs. 33\% for groups receiving CPX351 and 7+3, respectively. There are few examples of 
personalized treatment where a third chemotherapeutic is added to a chemotherapy regimen, tailored to a specific cytogenetic profile (Daver et al., 2020).

One of the few examples of a molecular lesion producing a distinct malignancy with analmost curative pharmacological agent is $\mathrm{t}(15: 17)$, a reciprocal translocation involving the retinoic acid receptor-alpha gene on chromosome 17 and the promyelocytic leukemia gene on chromosome 15. This molecular lesion results in acute promyelocytic leukemia and manifests in incomplete myeloid differentiation. All trans retinoic acid activates the resulting fusion oncoprotein to promote myeloid differentiation. For patients with the $t(15: 17)$ lesion, $7+3$ is not recommended; instead, all trans retinoic acid achieves complete remission in $90 \%$ of acute promyelocytic leukemia patients (Shipley \& Butera, 2009).

Compared to patients carrying a wild type FLT3, approximately $30 \%$ of AML patients carry either a FLT3 internal tandem duplication or tyrosine kinase domain mutation, conferring an inferior prognosis. A phase 3 trial recruited AML relapse patients with and without FLT3 mutations and provided gilteritinib, a FLT3 mutant inhibitor. Patients with mutated FLT3 exhibited an overall response of $49 \%$ compared to $12 \%$ in patients with wild type FLT3 (Daver et al., 2020). In relapsed AML patients with a FLT3 mutation, the FLT3 inhibitors quizartinib and gilteritnib demonstrated superior overall survival rates, compared to high intensity $7+3$ regimens. The FLT3 inhibitor midostaurin has also been approved to be used as a third agent alongside $7+3$, specifically for AML patients with a FLT3 mutation (Daver et al., 2020).

Taken together, $7+3$ or a liposomal formulation of its components are still the mainstay of anti-AML therapy, almost unchanged over the past four decades. In AML patients harboring a genetic lesion with a clinically approved inhibitor, clinicians may deviate from the standard $7+3$ 
therapy and provide the lesion-specific inhibitor as a single agent or in combination with $7+3$ (Daver et al., 2020).

\subsubsection{Treatment Related Co-Morbidities And Off-Targets Of 7+3 Therapy}

Ara-C targets DNA polymerase $\alpha$ while daunorubicin targets topoisomerase II, suppressing DNA synthesis required for rapid AML proliferation (Lichtman, 2013). At therapeutic doses, offtargets of these two agents include other hematopoietic cells, resulting in therapy-induced cytopenias, as well as cardiomyopathy (Khwaja et al., 2016). In addition to treatment-induced comorbidities, leukemia initiating cells evade chemotherapy-induced apoptosis by decreasing intracellular concentrations of the drug. For cytarabine, this can be accomplished by decreasing cellular ara-C uptake by decreasing drug transporters, downregulating the enzymes that activate the chemotherapeutic to its active form, upregulating the enzymes that break down the chemotherapeutic, or increasing levels of the target enzyme's substrate to outcompete the drug (Lamba, 2009).

Taken together, the primary disadvantages of the $7+3$ regimen include altered leukemia metabolism to evade intracellular chemotherapy accumulation and significant co-morbidities that prevent dose escalation in mature AML patients (Khwaja et al., 2016). This demonstrates the need for novel therapeutics that selective eliminate the leukemia population while being well-tolerated by normal healthy cells.

\subsection{Mitochondrial Oxidative Metabolism}

In 1956, Warburg observed an abrogation of the Pasteur effect, where lactate generation was inhibited in the presence of oxygen, in tumor ascites cells. Despite the presence of oxygen, tumor cells preferred to generate lactate - Warburg hypothesized that the mitochondria of tumor cells must have been defective or injured during the process of transformation. This observation 
was one of the first hypotheses regarding the role of mitochondrial metabolism in cancer (Heiden et al., 2009). In 1992, the interest in the role of mitochondrial oxidative metabolism in cancer was re-visited when the newly discovered mitochondrial B-cell lymphoma 2 (Bcl-2) protein family exerted tight control over cellular apoptosis (Certo et al., 2006).

Mitochondria are double membraned organelles, landmarked by the outer (OMM) and inner (IMM) mitochondrial membranes separated by an intermembrane space (IMS). The surface of the IMM is folded into cristae, tight folds that maximize surface area for metabolic processes. The IMM is impermeable to many metabolites without the assistance of a transporter enzyme. The space within the inner membrane is the mitochondrial matrix (Martínez-Reyes \& Chandel, 2020).

In both non-transformed and cancer populations, the tricarboxylic acid (TCA) cycle and oxidative phosphorylation (OXPHOS) are two metabolic pathways critical to mitochondrial oxidative metabolism (Figure 1.4.1)(Martínez-Reyes \& Chandel, 2020)(Sharabi \& Tsokos, 2020). Occurring in the matrix, the TCA cycle generates biosynthetic intermediates to fuel cell growth and proliferation (Martínez-Reyes \& Chandel, 2020). Occurring in the electron transport chain (ETC) that is anchored to the IMM, OXPHOS generates adenosine triphosphate (ATP), required for cell survival (Sharabi \& Tsokos, 2020). Glucose, fatty acid, and glutamine oxidation are the primary oxidative pathways feeding carbon atoms into the TCA cycle and electrons, via reductive equivalents of nicotinamide adenine dinucleotide (NAD+/NADH) and flavin adenine dinucleotide $\left(\mathrm{FAD} / \mathrm{FADH}_{2}\right)$, to OXPHOS (Figure 1.4.1) (Sharabi \& Tsokos, 2020). 


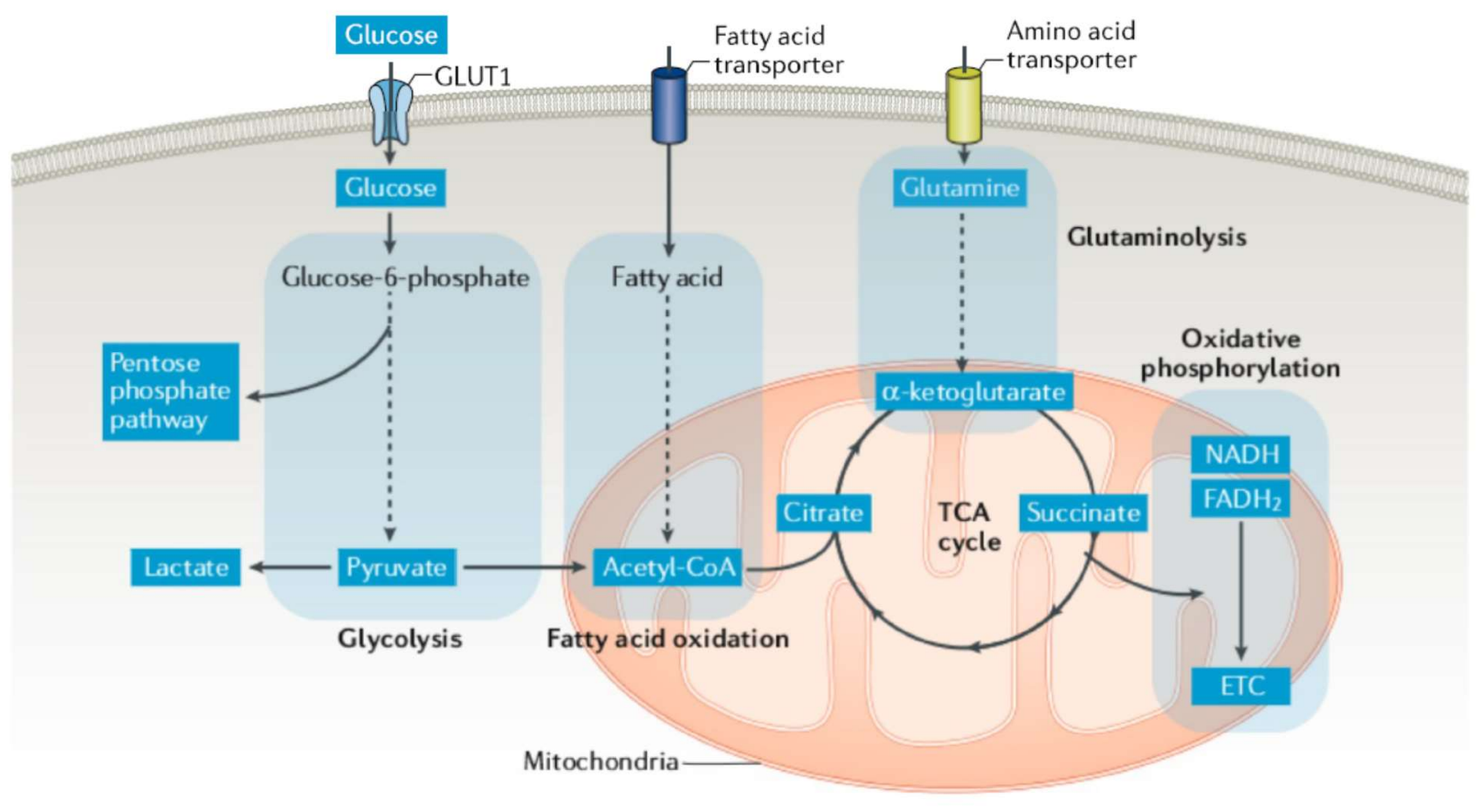

Figure 1.4.1 Oxidation of Glucose, Glutamine, And Fat Fuel Mitochondrial Oxidative Metabolism

This schematic highlights the oxidation of glucose, glutamine, and fatty acids supporting oxidative metabolism at the TCA cycle as well as OXPHOS at the ETC. In brief, glucose and fatty acids are oxidized to acetyl-CoA. Acetyl-CoA condenses with oxaloacetate and enters the TCA cycle as citrate. Glycolytic intermediates may be shunted away from glycolysis prior to oxidation to pyruvate towards the pentose phosphate pathway, a critical source of ribose-5-phosphate sugar. Glycolysis-derived pyruvate can also be converted to lactate, regenerating $\mathrm{NAD}^{+}$. Glutaminolysis provides $\alpha$-ketoglutarate to the TCA cycle. TCA cycle activity generates $\mathrm{NADH}$ and $\mathrm{FADH}_{2}$, fuelling OXPHOS at the ETC. TCA metabolites can also be shunted away prior to complete oxidation to fuel other pathways producing metabolites such as aspartate, NADPH. Abbreviations: GLUT1: glucose transporter 1; TCA: tricarboxylic acid cycle; NADH: reduced nicotinamide adenine dinucleotide; reduced $\mathrm{FADH}_{2}$ : flavin adenine dinucleotide; ETC: electron transport chain. Reprinted with permission (Sharabi \& Tsokos, 2020). 


\subsubsection{Tricarboxylic Acid (TCA) Cycle}

The TCA cycle occurs mostly in the mitochondrial matrix (Figure 1.4.1) and is comprised of a series of enzymes operating in sequence (citrate synthase, aconitase, isocitrate dehydrogenase (IDH), alpha-ketoglutarate dehydrogenase, succinyl-CoA synthetase, succinate dehydrogenase (also know as complex II), fumarase, and malate dehydrogenase). One cycle of these oxidative reactions produces carbon dioxide, and one molecule each of guanosine triphosphate, NADH, and $\mathrm{FADH}_{2}$ (Martínez-Reyes \& Chandel, 2020). Acetyl-coenzyme A (CoA) is a two-carbon long fat molecule coupled to the coenzyme A cofactor that can be derived from fatty acid oxidation (FAO) and glycolysis. Acetyl-CoA enters the TCA cycle via citrate synthase while alpha-ketoglutarate, derived from the amino acid glutamine, enters via alpha-ketoglutarate dehydrogenase (Figure 1.4.1)(Martínez-Reyes \& Chandel, 2020)(Sharabi \& Tsokos, 2020). TCA metabolites being oxidized in this cycle are also siphoned from the mitochondria to fuel other cellular purposes. For example, TCA-derived acetyl-CoA molecules are used to acetylate histones; the extent of histone acetylation alters cell immune, proliferation, differentiation processes (Martínez-Reyes \& Chandel, 2020). The gain of function mutations in IDH1 and IDH2 are found in about 20\% of AML patients, allowing conversion of alpha-ketoglutarate into the oncometabolite 2hydroxyglutarate. This oncometabolite acts as an alpha-ketoglutarate antagonist to inhibit several alpha-ketoglutarate-dependent dioxygenases modulating histone demethylation and immune cell activation (Reitman \& Yan, 2010). TCA-derived citrate is exported by dicarboxylate antiporter solute carrier family 25 and contributes to the cytosolic production of reduced nicotinamide adenine dinucleotide phosphate (NADPH), a critical antioxidant modulating cellular redox status. TCA-derived oxaloacetate is converted by aspartate aminotransferase to aspartate, a metabolite critical to nucleotide and protein biosynthesis (Martínez-Reyes \& Chandel, 2020). 


\subsubsection{Oxidative Phosphorylation (OXPHOS) At The Electron Transport Chain (ETC)}

Located on the IMM, OXPHOS generates ATP using ETC complexes I - V (Zhao et al., 2019). The reducing equivalents $\mathrm{NADH}$ and $\mathrm{FADH}_{2}$, which are produced from TCA cycling as well as from the oxidation of fatty acids, glucose, and amino acids, are oxidized at complexes I (NADH dehydrogenase) and II ( $\mathrm{FADH}_{2}$ dehydrogenase), respectively. Complex III (bc1 cytochrome c complex) receives electrons from two electron transfer agents. First, the protein ubiquinone (UQ) transfers electrons from complexes I and II to complex III. Secondly, the electron transfer flavoprotein (ETF) delivers electrons from multiple dehydrogenases involved in FAO to complex III, bypassing complexes I and II. The protein cytochrome c shuttles electrons from complex III to complex IV (cytochrome c oxidase). At complex IV, two electrons are rapidly passed to oxygen, the final electron acceptor - rapid protonation produces water. For every electron accepted at complexes I, III, IV, the free energy from the transfer allows one proton to be pumped from the mitochondrial matrix to the IMS, resulting in an electrochemical gradient that drives phosphorylation of adenosine diphosphate (ADP) to ATP at complex V. The electrochemical gradient, also known as the mitochondrial potential, is a hallmark measure of mitochondrial function. Depolarization of the mitochondrial potential is one of the first steps in cellular apoptosis (Zhao et al., 2019).

\subsubsection{Glycolysis}

Glycolysis occurs in the cytosol and, through a ten step reaction, results in the conversion of glucose to pyruvate (Figure 1.4.1)(Sharabi \& Tsokos, 2020)(Lunt \& Vander Heiden, 2011). Through this conversion, two net molecules each of ATP and NADH are produced (Lunt \& Vander

Heiden, 2011). Glycolysis is a critical cytosolic source for biosynthetic intermediates fueling metabolic pathways critical for cell division (Luengo et al., 2021). For example, glucose-6- 
phosphate can be diverted towards the pentose phosphate pathway, a critical source of pentose sugars and NADPH (Figure 1.4.1). Pentose sugars are used in nucleotide synthesis while NADPH is required for fatty acid, sterol, and ceramide synthesis (Sharabi \& Tsokos, 2020). Glycolysisderived 3-phosphoglycerate is a precursor metabolite for several amino acids including cysteine, glycine, and serine (Lunt \& Vander Heiden, 2011).

Glucose-derived pyruvate has two main fates depending on the proliferative and energy needs of the cell (Luengo et al., 2021)(Olson et al., 2016)(Jose et al., 2011). First, the cytosolic enzyme lactate dehydrogenase converts conversion of pyruvate to lactate, producing one molecule of $\mathrm{NAD}^{+}$(Figure 1.4.1). Secondly, mitochondrial enzyme pyruvate dehydrogenase (PDH) links glycolysis to the TCA cycle through the conversion of pyruvate to acetyl-CoA, producing one molecule of NADH (Sharabi \& Tsokos, 2020)(Olson et al., 2016). While oxidative carboxylation to pyruvate increases the ATP output, the metabolic conversion of glucose to lactate is $10-100$ times faster than mitochondrial metabolism; the ATP production through glycolysis is thus more kinetically favorable and critical under metabolic stress (Jose et al., 2011). In rapidly proliferating cells, the need for high levels of glycolytic intermediates, via elevated glycolytic flux, outstrips the need for ATP. In contrast to NADH, $\mathrm{NAD}^{+}$drives glycolysis, via positive feedback, to favor constant replenishment of glycolytic intermediates required for cell proliferation (Luengo et al., 2021). Taken together, glycolysis-linked lactate production, not pyruvate oxidation, is the preferred metabolic pathway to rapidly replenish $\mathrm{NAD}^{+}$and $\mathrm{ATP}$ for proliferating cells or for cells under energy stress (Luengo et al., 2021)(Olson et al., 2016)(Jose et al., 2011).

\subsubsection{Glutaminolysis}

Glutamine is the most abundant amino acid circulating in peripheral blood (Yang et al., 2017). Glutaminolysis involves the conversion of glutamine to alpha-ketoglutarate via a glutamate 
intermediate in the mitochondria (Figure 1.4.1). In the mitochondria, glutamate is converted by glutamate dehydrogenase to TCA-bound alpha-ketoglutarate, producing one molecule of NADH (Figure 1.4.1)(Sharabi \& Tsokos, 2020)(Yang et al., 2017). TCA oxidation of glutamine-derived alpha-ketoglutarate generates additional NADH and $\mathrm{FADH}_{2}$ to support AML respiration (Jacque

et al., 2015). Glutamine-derived ammonia is also a critical source of nitrogen for the synthesis of non-essential amino acids such as aspartate, alanine, and phosphoserine (Yang et al., 2017).

\subsection{Fatty Acid Oxidation (FAO)}

Mitochondrial fatty acid oxidation is the primary pathway in mammalian cells for catabolizing saturated fatty acids below 18 carbons in length (Wanders et al., 2010)(Bartlett \& Eaton, 2004). FAO produces TCA-bound acetyl-CoA and one molecule each of NADH and $\mathrm{FADH}_{2}$, bound for OXPHOS (Figure 1.5.1). There are three separate stages of FAO involving, first, the entry of fat into the cytosol, second, the transport of fat through the mitochondrial membranes, and third, the successive shortening of the fat within the mitochondrial matrix (Figure 1.5.1, Table 1.5.1)(Houten \& Wanders, 2010). 


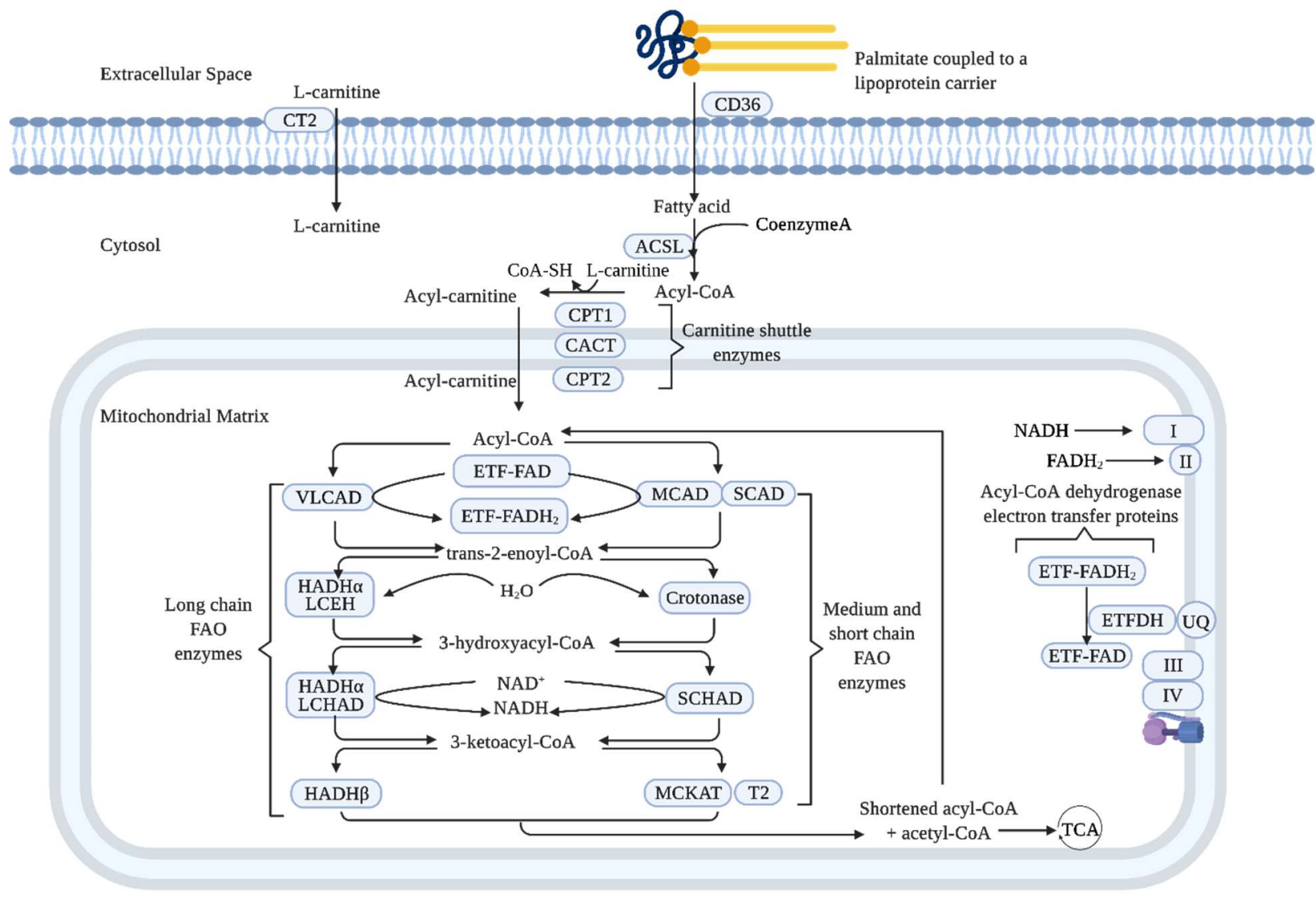

Figure 1.5.1 Mitochondrial Fatty Acid Oxidation

The schematic exhibits the mitochondrial fatty acid oxidation pathway in humans (Houten \& Wanders, 2010). Table 1.5.1 (below) summarizes the full protein name, short form alias, and gene of each FAO enzyme in this figure. Abbreviations: ETC, electron transport chain; I, ETC complex I; II, ETC complex II; III, ETC complex III; IV, ETC complex IV; UQ, ubiquinone; TCA, tricarboxylic acid cycle (Houten \& Wanders, 2010). Created in Biorender. 
Table 1.5.1 List Of Protein Names, Short Form Aliases, And The Encoding Genes For The Intramitochondrial FAO Enzymes

\begin{tabular}{|c|c|c|}
\hline \multicolumn{3}{|c|}{ Enzymes of mitochondrial FAO } \\
\hline Protein name & Protein short form alias & Gene \\
\hline \multicolumn{3}{|c|}{ Enzymes catalyzing cytosolic entry of fat from extracellular space } \\
\hline Organic cation/carnitine transporter 2 & CT2 & OCT2 \\
\hline Cluster of differentiation 36 & CD36 & $C D 36$ \\
\hline Acyl-CoA synthetase & ACSL & $S L C 22 A 5$ \\
\hline \multicolumn{3}{|c|}{ Enzymes of the carnitine shuttle } \\
\hline Organic cation/carnitine transporter 2 & $\mathrm{CT} 2$ & CT2 \\
\hline Carnitine palmitoyltransferase 1 & CPT1 & CPT1 \\
\hline Carnitine acylcarnitine translocase & CACT & SLC25A20 \\
\hline Carnitine palmitoyltransferase 2 & CPT2 & CPT2 \\
\hline \multicolumn{3}{|c|}{ Enzymes catalyzing long chain FAO } \\
\hline Very long chain acyl-CoA dehydrogenase & VLCAD & $A C A D V L$ \\
\hline $\begin{array}{l}\text { Mitochondrial trifunctional protein, alpha subunit } \\
\text { Long chain enoyl-CoA hydratase }\end{array}$ & $\begin{array}{l}\text { HADH } \alpha \\
\text { LCEH }\end{array}$ & $H A D H \alpha$ \\
\hline $\begin{array}{l}\text { Mitochondrial trifunctional protein, alpha subunit } \\
\text { Long chain hydroxylacyl-CoA dehydrogenase }\end{array}$ & $\begin{array}{l}\text { HADH } \alpha \\
\text { LCHAD }\end{array}$ & $H A D H \alpha$ \\
\hline $\begin{array}{l}\text { Mitochondrial trifunctional protein, beta subunit, } \\
\text { Long chain 3-ketothiolase }\end{array}$ & HADH $\beta$ & $H A D H \beta$ \\
\hline \multicolumn{3}{|c|}{ Enzymes catalyzing medium and short chain FAO } \\
\hline Medium-chain acyl-CoA dehydrogenase & MCAD & $A C A D M$ \\
\hline Medium-chain 3-ketoacyl-CoA thiolase & MCKAT & $A C A A 2$ \\
\hline Short-chain acyl-CoA dehydrogenase & SCAD & $A C A D S$ \\
\hline Short-chain enoyl-CoA hydratase & Crotonase & ECHS1 \\
\hline Short-chain (S)-3-hydroxyacyl-CoA dehydrogenase & SCHAD & $H A D H$ \\
\hline Acetoacetyl-CoA thiolase & $\mathrm{T} 2$ & ACAT1 \\
\hline \multicolumn{3}{|c|}{ Enzymes shuttling electrons from the ACADs to the ETC } \\
\hline Electron Transfer Flavoprotein & ETF & $E T F A, E T F B$ \\
\hline Electron Transfer Flavoprotein Dehydrogenase & ETFDH & $E T F D H$ \\
\hline
\end{tabular}




\subsubsection{Cytosolic Entry Of Fat From Extracellular Space}

FAO enzymes are substrate-length specific. Different enzyme isoforms will catalyze the same reaction depending on aliphatic chain length: long (14-18 carbons), medium (six-12 carbons), or short (four carbons) fats (Figure 1.5.1, Table 1.5.1) (Bartlett \& Eaton, 2004). In humans, dietary fats traveling in peripheral blood are packaged into lipoprotein complexes for solubility (Havel, 1987). The entry of long chain fatty acids into the cytosol is governed by the cell surface transporter CD36 while medium and short chain fats may diffuse through the cell membrane without the assistance of a fat transporter. In the cytosol, fatty acids are rapidly activated for oxidation by esterification to a CoA cofactor, forming an acyl-CoA; this reaction is carried out by a length specific isoform of the acyl-CoA synthetase (ACSL) family of enzymes (Wanders et al., 2010).

\subsubsection{Carnitine Shuttle In The Mitochondrial Membrane}

Long chain acyl-CoA's are unable to diffuse freely through the OMM; the carnitine shuttle assists in fat transport across the OMM and consists of three enzymes operating in rapid succession: carnitine palmitoyl transferase 1 (CPT1), carnitine acyl-carnitine translocase (CACT), and the carnitine palmitoyl transferase 2 (CPT2) (Figure 1.5.1, Table 1.5.1). The carnitine cofactor allows transport of fats through the mitochondrial membrane and is imported into the cell by the enzyme organic cation/carnitine transporter 2 (CT2) (Wanders et al., 2010). On the OMM surface facing the cytosol, CPT1 trans-esterifies the acyl-CoA with a carnitine cofactor to produce an acyl-carnitine. Embedded within the mitochondrial membrane, the CACT exchanges one carnitine cofactor from the matrix to the cytosol for the entry of one acyl-carnitine into the mitochondrial matrix. Anchored to the IMM and facing the matrix, CPT2 performs the reverse of CPT1, converting the acyl-carnitine intermediate back to an acyl-CoA (Bartlett \& Eaton, 2004). 
In the matrix, acyl-CoA's are broken down by $\beta$-oxidation, a four-step repeating cycle that successively shorten fats by two carbons per cycle (Houten \& Wanders, 2010).

\subsection{3 ß-Oxidation Cycle Within The Mitochondrial Matrix}

When a saturated fat enters the matrix, the first step of the cycle is dehydrogenation of the acyl-CoA to a trans-2-enoyl-CoA by a length specific acyl-CoA dehydrogenase isoform (Figure 1.5.1). The second step is hydration of the trans-2-enoyl-CoA to a (S)-3-hydroxyacyl-CoA by a length specific enoyl-CoA hydratase. The third step involves the dehydrogenation of the $(S)-3-$ hydroxyacyl-CoA to a 3-ketoacyl-CoA by a length specific (S)-3-hydroxyacyl-CoA dehydrogenase. Finally, a length specific 3-ketoacyl-CoA thiolase cleaves the fat at $C-3$ to produce one TCA-bound molecule of acetyl-CoA and a fat shortened by two carbons. While this four step cycle does not directly generate ATP, steps one and three supply electrons to the ETC. In step one, the ACAD transfers an electron to a FAD cofactor attached to the ETF (Houten \& Wanders, 2010). In step three, the $(S)$-3-hydroxyacyl-CoA dehydrogenase reduces a molecule of $\mathrm{NAD}^{+}($Houten \& Wanders, 2010).

The enzymes catalyzing long chain FAO are: the very long chain acyl-CoA dehydrogenase (VLCAD) and the alpha and beta subunits of the mitochondrial tri-functional protein (MTP)(Figure 1.5.1, Table 1.5.1). The MTP is an enzyme complex with an alpha (HADH $\alpha$ ) and a beta $(\mathrm{HADH} \beta)$ subunit. An alpha subunit consisting of the long chain enoyl-CoA hydratase (LCEH) and long chain hydroxyacyl-CoA dehydrogenase (LCHAD) enzymes, catalyzing steps

two and three for long chain fats (Bartlett \& Eaton, 2004). The beta subunit is the long chain 3ketoacyl-CoA thiolase (LCKAT), catalyzing step four of the FAO cycle for long chain fats. These enzymes catalyzing long chain FAO are anchored to the IMM, facing the matrix, and are clustered in close proximity to the ETC, facilitating rapid electron transfer to support OXPHOS (Bartlett \& 
Eaton, 2004). Once saturated long chain fats are sufficiently shortened by long chain FAO enzymes to 12 carbons, shortened acyl-CoA's are then processed by downstream medium FAO enzymes (Wanders et al., 2010).

In contrast to long chain FAO enzymes, medium and short chain FAO enzymes are solubilized in the matrix and have the highest specificity for medium and short chain acyl-CoA's, respectively (Figure 1.5.1, Table 1.5.1). The first reactions for medium and short length saturated acyl-CoA's are processed by the medium (MCAD) and short chain acyl-CoA dehydrogenases (SCAD), isoforms of the acyl-CoA dehydrogenase (ACAD) family. The second, third, and fourth steps of medium and short chain FAO are not catalyzed by a single tri-functional enzyme but are catalyzed by discrete, individual enzymes. Crotonase and the short chain $(S)$-3-hydroxyacyl-CoA dehydrogenase-CoA dehydrogenase (SCHAD) catalyzes the second and third reactions for medium and short chain fats. The fourth reaction is carried out by 3-ketoacyl-CoA thiolase (MCKAT) for medium length fats and by acetoacetylthiolase (T2) for short length fats (Wanders et al., 2010). FAO-derived acetyl-CoA is then completely oxidized to $\mathrm{CO}_{2}$ by isocitrate and alphaketoglutarate dehydrogenase in the TCA cycle (Houten \& Wanders, 2010)(Wanders et al., 2010).

\subsection{Mitochondrial Properties Of AML And Hematopoietic Cells}

The leukemia blast and stem cell populations exhibit altered mitochondrial characteristics, which distinguishes them from the normal hematopoietic population (Samudio et al., 2010)(Kuntz et al., 2017). While the hematopoietic stem population relies primarily on anaerobic glycolysis to maintain quiescence (Filippi \& Ghaffari, 2019), the leukemia population demonstrates abnormally increased rates of mitochondrial metabolism and is more dependent on OXPHOS, supported primarily by FAO, for ATP and cell survival (Nakahara et al., 2014). Besides fat, AML and 
hematopoietic populations also oxidize amino acids such as glutamine to supply electrons to the ETC, fuelling OXPHOS and provides metabolites bound for the TCA cycle (Jones et al., 2018).

OXPHOS activity can be assessed by measuring oxygen flux using a high resolution respirometer. In intact cells, the basal rate of oxygen flux refers to the amount of oxygen consumed by the mitochondria fuelled by substrates in a non-metabolically stressed state. The maximal rate of oxygen flux refers to the maximum amount of oxygen the mitochondria can consume in response to a metabolically stressed state induced by substrate starvation or pharmacological insult. The spare reserve capacity is the difference between the maximal and basal rates of oxygen flux and reflects the extent that cells can increase their mitochondrial metabolism to when under metabolic stress (Hutter et al., 2006).

Previously, AML cells demonstrated higher oxygen flux rates, compared to normal hematopoietic cells, while exhibiting a lower spare reserve capacity (Sriskanthadevan et al., 2015). This increased oxygen flux is supported by increased mitochondria mass and not a concomitant increase in overall ETC activity, rendering AML populations sensitive to inhibitors of mitochondrial translation and protein synthesis (Škrtić et al., 2011)(Mirali et al., 2020)(Seneviratne et al., 2020). Compared to normal hematopoietic cells, a decreased spare reserve capacity suggested the AML population was more vulnerable to pharmacological agents targeting mitochondrial oxidative pathways (Sriskanthadevan et al., 2015). Targeting oxidative pathways that support OXPHOS such as FAO (German et al., 2016)(Shafat et al., 2017) or by directly targeting ETC complexes (Pollyea et al., 2018)(Molina et al., 2018)(Baccelli et al., 2019) exploits a mitochondrial dependency unique to AML and presents a therapeutic window for targeting AML metabolism. Besides fuelling the TCA cycle and ETC activity, FAO activity also governs differentiation and proliferation in both AML and hematopoietic cells. 
To provide a lifetime supply of functional blood cells, HSCs must maintain quiescence by utilizing anaerobic glycolysis and minimizing mitochondrial oxidative metabolism (Filippi \& Ghaffari, 2019)(Ito et al., 2019). When the HSC population must divide, anaerobic glycolysis is transiently suppressed and FAO is increased to promote asymmetric division. Asymmetric division produces a single stem cell identical to the parent HSC cell and one daughter progenitor cell that is more committed to differentiation with higher proliferative potential. Asymmetric division ensures maintenance of the HSC pool while providing more mature cells that will differentiate and proliferate into functional myeloid cells. Taken together, the metabolically flexible HSC pool determines cell fate by switching between fat and glucose oxidation, in response to physiological demands for functional myeloid cells (Filippi \& Ghaffari, 2019)(Ito et al., 2019).

Certain leukemia subpopulations exhibit increased rates of either glucose (Pardee et al., 2018), glutamine (Jones et al., 2018), or fat oxidation (German et al., 2016)(Shafat et al., 2017) with a decreased capacity to switch to the oxidation of an alternative substrate upon inhibition of the upregulated pathway to avert an energy crisis and cell death (Molina et al., 2018)(Lagadinou et al., 2013)(Sharon et al., 2019). Pharmacological targeting of the overreliance on a preferred mitochondrial oxidative pathway and an inability to adapt to pharmacological inhibition selectively eliminates AML, while sparing normal hematopoietic cells. This highlights a metabolic vulnerability in AML (Molina et al., 2018)(Lagadinou et al., 2013)(Sharon et al., 2019).

In certain AML subpopulations, glutamine withdrawal (Jones et al., 2018) and inhibition of ETC complexes I (Molina et al., 2018) and II (Pollyea et al., 2018) (Sharon et al., 2019) failed to trigger an increase in compensatory glycolysis, resulting in ATP depletion and AML death. In the malignant population, ectopic overexpression of glycolytic enzymes (Molina et al., 2018) or pharmacological activation of glycolysis-supported lactate production by the integrated stress 
response inhibitor (ISRIB) (Sharon et al., 2019) was required to prevent ATP depletion and cell death. In contrast, normal cells demonstrated a high glycolytic capacity and survived inhibition of mitochondrial metabolism (Lagadinou et al., 2013). In support of a therapeutic window, normal hematopoietic cells demonstrate metabolic flexibility when determining cell fate and were spared by inhibitors of mitochondrial metabolism (Kuntz et al., 2017)(Lagadinou et al., 2013). Taken together, compared to normal hematopoietic cells, certain AML populations are metabolically rigid, demonstrating an overreliance on either glucose, fat, or amino acids to support mitochondrial metabolism and a decreased capacity to increase alternative pathways upon pharmacological stress, resulting in an energy crisis that leads to cell death.

\subsection{Mitochondrial FAO As An Anti-AML Target}

Compared to normal hematopoiesis, cell-surface FAO enzymes, such as apolipoprotein-CII (APOC2)(Zhang et al., 2020), CD36(Ye et al., 2016) and CT2(Wu et al., 2015), cytosolic FAO enzymes, such as fatty acid binding protein 4 (FABP4) (Shafat et al., 2017), as well as mitochondrial-surface FAO enzymes, such as prolyl hydroxylase dehydrogenase 3 (PHD3) and CPT1, were increased in AML and were critical to mitochondrial oxidative metabolism (Figure

1.7.1). While sparing normal hematopoietic cells, the loss of the activity of these FAO enzymes by genetic knockdown or pharmacological inhibition resulted in enhanced sensitivity to induction chemotherapy and decreased AML survival, proliferation, clonogenic growth, and engraftment capacity (German et al., 2016)(Shafat et al., 2017) (Samudio et al., 2010). 

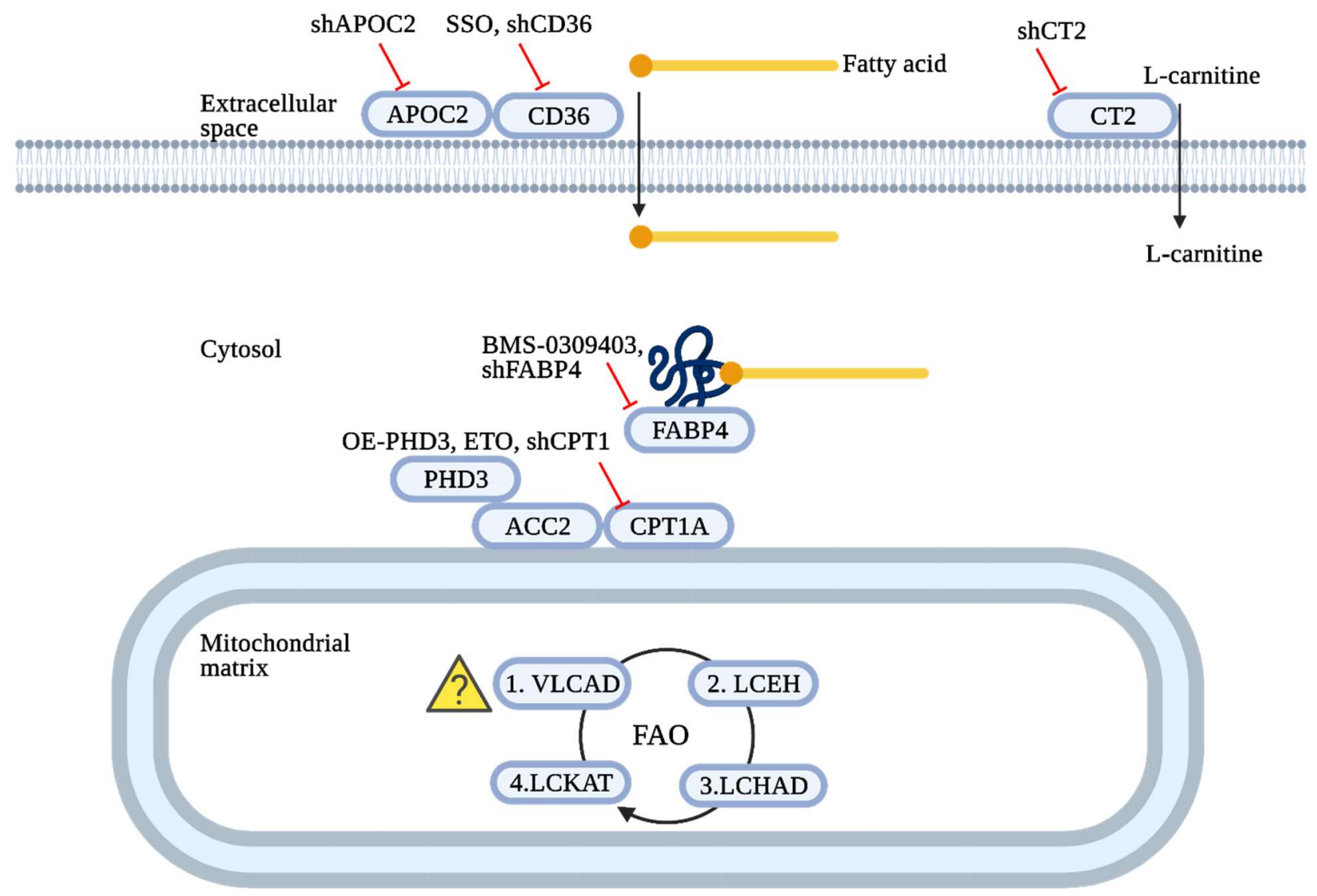

\section{Figure 1.7.1 FAO Inhibition In AML}

The schematic summarizes the inhibition of cell surface, cytosolic, mitochondrial surface FAO targets by genetic ablation via shorthair pin RNA (shRNA) and pharmacological inhibition resulting in decreased AML survival, proliferation, clonogenic growth, and engraftment capacity (German et al., 2016)(Shafat et al., 2017)(Zhang et al., 2020)(Wu et al., 2015). Full enzyme names for the enzyme short form designations (CD36, CT2, CPT1A, VLCAD, LCEH, LCKAT, LCHAD) are tabulated in Table 1.5.1. Abbreviations: shAPOC2: shRNA targeting APOC2; SSO: sulfosuccinimidyl oleate; shCD36: shRNA targeting CD36; shCT2: shRNA targeting CT2; BMS0309304: 2-[[2'-(5-Ethyl-3,4-diphenyl-1 $H$-pyrazol-1-yl)[1,1'-biphenyl]-3-yl] oxy]-acetic acid; FABP4: fatty acid binding protein 4; shFABP4: shRNA targeting FABP4; OE-PHD3: ectopic overexpression of PHD3; ETO: etomoxir; shCPT1: shRNA targeting CPT1; ACC2: acetyl-CoA carboxylase 2. The question mark in the mitochondrial matrix, in yellow, highlights the four intramitochondrial enzymes of long chain FAO which have not been previously studied in AML. Created in Biorender. 
On the OMM surface, ACC2 regulates FAO by producing malonyl-CoA, an endogenous inhibitor of CPT1. ACC2 undergoes activating hydroxylation by PHD3, acting as a metabolic brake on FAO (See PHD3, ACC2, CPT1: Figure 1.7.1)(German et al., 2016). In patient-derived AML samples and cell lines, PHD3 was constitutively under-expressed, resulting in higher rates, increased dependency, and a loss of repressive control on mitochondrial FAO. In cell lines with high PHD3 expression, PHD3 knockdown increased FAO activity and sensitivity to pharmacological inhibition by ETO. Ectopic overexpression of PHD3 resulted in decreased proliferation, clonogenic growth, and engraftment capacity in AML, confirming that FAO supports AML progression (See OE-PHD3: Figure 1.7.1). This study demonstrated that PHD3 control of FAO is dysregulated in AML and that FAO fuels AML energy metabolism, proliferation, clonogenic growth, and engraftment capacity (German et al., 2016).

A subpopulation of patient-derived leukemia cells overexpressing CD36 were resistant to cytarabine by sequestering within adipose tissue (See CD36: Figure 1.7.1). Within gonadal adipose tissue, these CD36+ AML cells secreted pro-inflammatory cytokines, such as interleukin (IL)-1 $\alpha$, IL-1 $\beta$, IL-6, tumor necrosis factor (TNF)- $\alpha$, to trigger dysregulated lipolysis from adjacent adipocytes, fueling leukemia FAO. Genetic ablation or pharmacological inhibition of CD36 by SSO restored sensitivity to ara-C (See shCD36 and SSO: Figure 1.7.1). This study demonstrated CD36 as a potential pharmacological target and that the AML population hijacks adjacent adipocytes to fuel leukemia FAO and evade chemotherapy (Ye et al., 2016).

The protein APOC2 was overexpressed in AML and is an upstream activator of CD36 (See APOC2 and CD36: Figure 1.7.1). Ectopic overexpression of either APOC2 or CD36 or both proteins simultaneously resulted in increased cell proliferation and clonogenic growth in AML cell lines and primary patient samples. In contrast, knockdown of either APOC2 or CD36 or both 
proteins simultaneously as well as pharmacological inhibition of CD36 by SSO suppressed energy metabolism and decreased AML cell proliferation, clonogenic potential, and engraftment capacity (See SSO: Figure 1.7.1)(Zhang et al., 2020).

Knockdown of CT2 suppressed FAO activity and decreased AML proliferation as well as clonogenic growth in AML cell lines (See shCT2: Figure 1.7.1). Genetic ablation of CT2 did not trigger an increase in glycolysis linked lactate production, further confirming that certain AML subpopulations are metabolically rigid and cannot upregulate compensatory pathways to avert ATP depletion. While CT2 genetic ablation hindered AML, no widely available pharmacological inhibitor of CT2 exists (See shCT2: Figure 1.7.1)(Wu et al., 2015).

Fatty acid binding protein 4 (FABP4), a lipid chaperone exclusively expressed in adipocytes, transferred fat molecules to AML cells in adipocyte-coculture (See FABP4: Figure 1.7.1). To model the hematopoietic bone marrow niche, AML cells grown in co-culture with adipocytes released pro-inflammatory cytokines (such as TNF- $\alpha$ ), triggering upregulation of FABP4 in adipocytes to deliver fat to AML cells. Increased fat transfer from adipocytes and CPT1 upregulation increased mitochondrial oxidative metabolism in AML fuelled by increased FAO activity (See CPT1: Figure 1.7.1)(Shafat et al., 2017). A separate study determined that high CPT1A expression was associated with decreased event free AML patient survival (Shi et al., 2016). Genetic knockdown of FABP4 or CPT1A and pharmacological inhibition by BMS0309403 and ETO, respectively, inhibited FAO and mitochondrial respiration, further confirming the critical role of long chain FAO in supporting AML metabolism (See shFABP4, shCPT1, BMS0309403, ETO: Figure 1.7.1)(Shafat et al., 2017). Mice carrying AML patient-derived cells with either a FABP4 or CPT1A knockdown demonstrated increased survival, compared to mice carrying the same cells with a scramble control. In agreement with a previous study (Ye et al., 
2016), this study demonstrated that FAO was critical to AML engraftment and that AML cells remodel the surrounding niche to favor leukemia progression and disrupt hematopoiesis (Shafat et al., 2017).

To more accurately model AML relapse, NSG mice engrafted with patient-derived AML cells were treated with ara-C daily for five consecutive days, mimicking $7+3$ induction therapy received by human AML patients. Compared to de novo AML cells that were not exposed to araC, residual leukemia cells that survived ara-C therapy demonstrated increased mitochondrial mass, increased oxidative stress, highly polarized mitochondrial membrane potential, CD36 overexpression and increased FAO activity (See CD36: Figure 1.7.1). Pharmacological inhibition of FAO with ETO decreased oxygen consumption and re-sensitized relapse initiating cells to araC-induced death; this study demonstrated that residual leukemia cells surviving ara-C rely on increased FAO for survival and that FAO inhibition ablates the high mitochondrial oxidative activity that is characteristic of ara-C resistance (See ETO: Figure 1.7.1) (Farge et al., 2017).

In vitro, a novel anti-AML combination therapy consisted of venetoclax, an inhibitor of the anti-apoptotic protein Bcl-2, and azacytidine, a nucleoside analogue, selectively eliminated leukemia initiating cells by targeting the ETC CII (Pollyea et al., 2018)(Stevens et al., 2020). Clinical use of these agents as monotherapies demonstrated modest remission rates due to the rapid onset of chemoresistance (Jonas \& Pollyea, 2019). In a phase 1 trial consisting of de novo AML patients above the age of 65 who were ineligible for dose escalation of the traditional $7+3$ induction regimen, a combination of venetoclax and azacytidine achieved complete remission in $67 \%$ of patients and was well-tolerated in mature patients (Pollyea et al., 2020). Leukemia cells surviving this combination therapy upregulated FAO and subsequently initiated relapse. Pharmacological or genetic inhibition of FAO enzymes $\mathrm{CD} 36$ and $\mathrm{CPT} 1$ in vitro restored sensitivity to the 
combination, resulting in the elimination of AML cells resistant to the venetoclax/azacytidine combination (See shCD36, SSO, shCPT1, ETO: Figure 1.7.1)(Stevens et al., 2020).

AML cells surviving two distinct frontline anti-AML chemotherapy regimens upregulated FAO activity, confirming that FAO confers a pro-survival advantage to AML cells inducing relapse (Farge et al., 2017)(Stevens et al., 2020). In vitro FAO inhibition eliminated these resistant cells; further in vivo studies are required to assess if FAO inhibitors in combination with clinical regimens reduces AML relapse, the primary cause of AML patient death. Taken together, these studies demonstrated that the inhibition of various cell-surface, cytosolic, and mitochondrialsurface FAO enzymes by genetic knockdown or pharmacological blockade is detrimental to AML cell survival, proliferation, clonogenic growth, and engraftment capacity.

While AML studies to date have primarily focused on inhibition of overexpressed cellsurface or mitochondrial-surface FAO enzymes, the gene expression of four intramitochondrial proteins catalyzing long chain FAO in the mitochondrial matrix and their respective roles in AML oxidative metabolism have not previously studied (See VLCAD, LCEH, LCHAD, LCKAT: Figure 1.7.1). Ranolazine (RAN) and trimetazidine (TMZ) are both FAO inhibitors previously shown to target the fourth step of long chain FAO in the matrix which is catalyzed by the MTP $\beta$ subunit (Kantor et al., 2000)(Nash \& Nash, 2008). In AML, RAN and TMZ, at millimolar concentrations, were unable to induce AML death directly and only sensitized AML cells to induction chemotherapy. Given the lack of anti-AML efficacy resulting from MTP inhibition in these previous studies (Samudio et al., 2010)(German et al., 2016), the ACAD family of enzymes, catalyzing the first reaction of the four step intramitochondrial FAO cycle, has remained unexplored in AML and will be further examined in this thesis. 


\subsection{Acyl-CoA Dehydrogenases (ACADs)}

There are three ACAD isoforms responsible for mitochondrial FAO in humans (Houten \& Wanders, 2010)(Wanders et al., 2010)(Bartlett \& Eaton, 2004). The very long (VLCAD), medium (MCAD), and short chain (SCAD) ACADs optimally oxidize saturated acyl-CoA's that are 16, 8 and 4 carbons in length, respectively (Houten \& Wanders, 2010). AML studies investigating FAO have concentrated on the oxidation of long chain fats (Samudio et al., 2010)(German et al., 2016)(Shafat et al., 2017)(Farge et al., 2017)(Stevens et al., 2020). While many structural and biochemical features are conserved across the ACAD family, the remainder of this literature review will specifically focus on very long chain acyl-CoA dehydrogenase (VLCAD), the ACAD isoform that processes long chain fats (14-18 carbons long)(Bartlett \& Eaton, 2004). Out of all the long chain FAO enzymes, the role of VLCAD in AML mitochondrial metabolism has not been previously elucidated.

\subsubsection{VLCAD Expression}

ACADs are encoded by nuclear DNA, translated in the cytosol, and folded into functional enzymes in the mitochondria (McAndrew et al., 2008). In particular, the gene encoding for VLCAD is 5.4 kilobases long and is located on chromosome 17 between bands p11.2 and 11.13105 (McAndrew et al., 2008). The VLCAD cDNA encodes a polypeptide 655 amino acids long, consisting of a 615 amino acid long mature protein and a 40 amino acid long leader peptide (Schiff et al., 2013). The functional VLCAD is folded into a $71 \mathrm{kDa}$ polypeptide (Schiff et al., 2013). While all ACADs feature high sequence and fold similarity in the first 400 amino acids at the amino terminus, the polypeptide encoding for VLCAD is unique from other ACADs with an additional 180 amino acid residues at the carboxy terminus (McAndrew et al., 2008). 


\subsubsection{VLCAD Structure And Function}

At the N-terminus, the polypeptide that will fold into a functional VLCAD consists of an $\alpha$ helix domain, followed by a $\beta$-sheet domain and a second $\alpha$ helix domain (Figure 1.8.1 A)(Schiff et al., 2013). At the C-terminus, 180 amino acids are organized into a third $\alpha$ helix perpendicular to the N-terminus $\alpha$ helices (Figure 1.8.1 A)(Schiff et al., 2013). VLCAD exists as a homo-dimer bound to the IMM, in close proximity to the MTP and ETC complexes (Wang et al., 2019). The unique 180 amino acids near the carboxy terminus are critical for anchoring VLCAD to the IMM; in contrast, MCAD and SCAD are matrix-soluble tetramers (Schiff et al., 2013). 

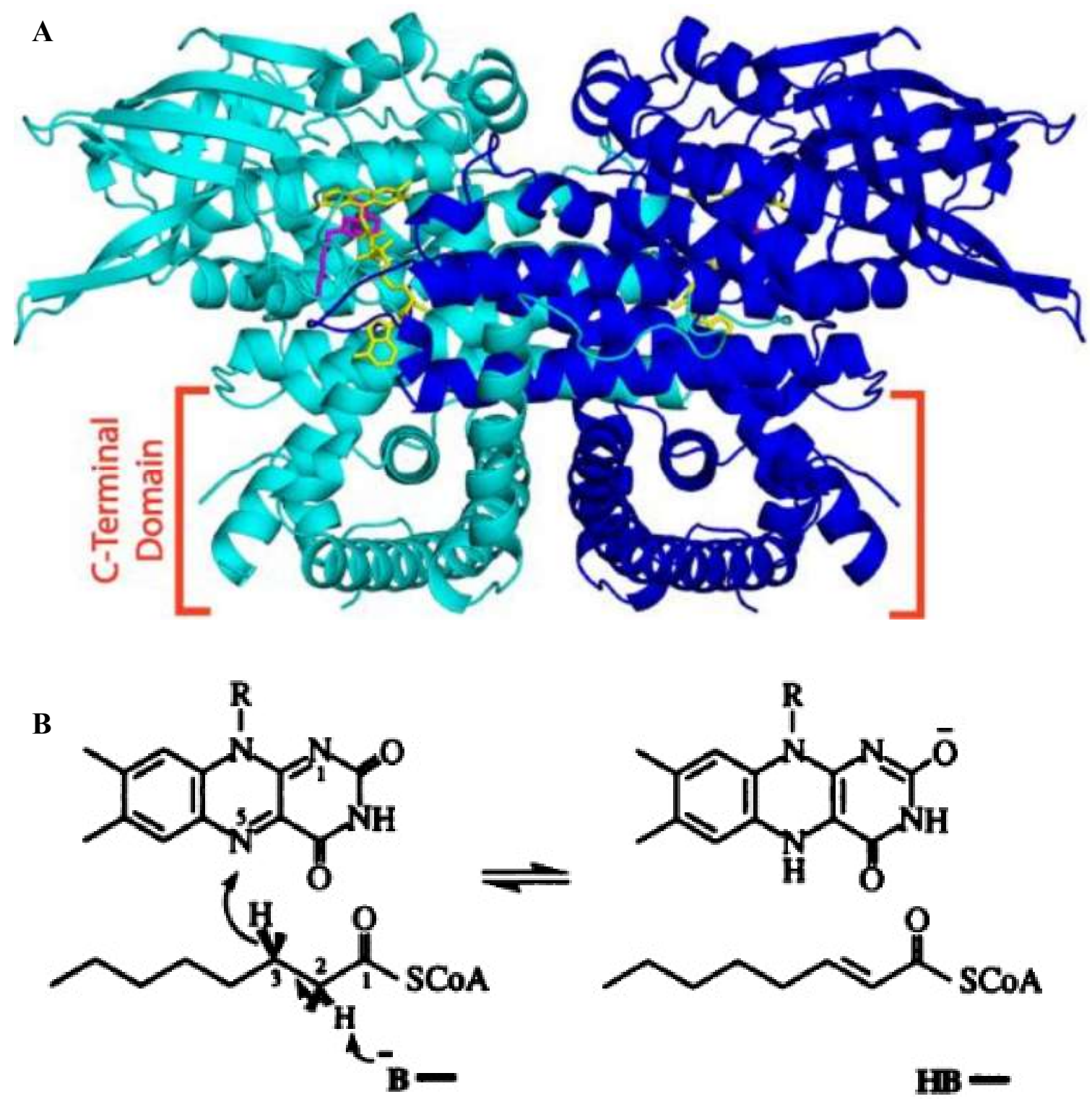

Figure 1.8.1 ACADs catalyze the oxidation of a fat by introducing an unsaturation across the C2-C3 bond

(A) The ribbon diagram showing the folding of the human VLCAD dimer composed of monomers that are highlighted in cyan and blue. The FAD cofactor of the VLCAD and the fourteen carbon atom long myristic acid as a substrate are highlighted in yellow and magenta, respectively. The Cterminus, responsible for anchoring VLCAD to the IMM, is marked with orange brackets. Reproduced from an open access article (McAndrew et al., 2008). (B) Reaction scheme for a dehydrogenation carried out by an ACAD isoform. The scheme highlights the initiating abstraction of the fat molecule's pro- $R$ proton by the catalytic glutamic acid (Represented as " $\mathrm{B}^{- \text {" }}$ at the bottom of the scheme) residue conserved in VLCAD, MCAD, and SCAD. This is followed by a hydride transfer to the ACAD's FAD cofactor, which is situated above the fat molecule in this scheme. Reproduced with permission (Thorpe \& Kim, 1995). 
In short, VLCAD catalyzes a dehydrogenation reaction across $C-2$ and $C-3$, also known as the $\alpha$ and $\beta$ carbons, of a saturated acyl-CoA 14 to 18 carbon atoms in length (Figure 1.8.1 B). ACADs contain a bound FAD cofactor that will transfer electrons directly to ubiquinone via the ETF (Figure 1.8.1 B). The ETF is also equipped with a FAD cofactor that receives an electron from an ACAD oxidizing a fat molecule (McAndrew et al., 2008). The reduced ETF is then oxidized by ETF dehydrogenase (ETFDH), passing the electron directly to ubiquinone while bypassing ETC complexes I and II to support OXPHOS (Wang et al., 2019).

The first step of the reaction involves the docking of a lipid 14-18 carbons in length into VLCAD's acyl-CoA binding pocket (See the fourteen carbon long myristic acid highlighted in cyan: Figure 1.8.2 A, B). Within VLCAD's acyl-binding pocket, glutamate-422 (Glu-422), VLCAD's catalytic residue, is situated in close proximity to the isoalloxazine ring of the FAD cofactor and near the acyl substrate's $\mathrm{C} 2-\mathrm{C} 3$ bond (Figure 1.8.1 B). Positioning of the catalytic residue is critical down to mere angstroms in length - genetic replacement of the glutamate residue with an aspartate residue results in 95\% loss of activity. The positioning of Glu-422's carboxylate moiety and the FAD cofactor is critical to proper polarization of the substrate's thioester bond. The pKa of a proton associated with $\alpha$-carbon's $\mathrm{C}-\mathrm{H}$ bond is $>20$ on an unbound fat molecule. Hydrogen bonding within the acyl-binding pocket lowers the pKa to 8-9, allowing the reaction to proceed (Figure 1.8.1 B)(Schiff et al., 2013). Once the fat is bound, the dehydrogenation will proceed with the Glu- 422 residue abstracting the pro- $R$ proton from $\alpha$-carbon's $\mathrm{C}-\mathrm{H}$ bond followed by a hydride transfer from the $\beta$ carbon to $\mathrm{N}(5)$ of the flavin ring (Figure 1.8.1 B). The bound FAD cofactor then reduces an oxidized ETF. The reduced ETF then transfers the electrons to ubiquinone, contributing to OXPHOS (Schiff et al., 2013). 
A

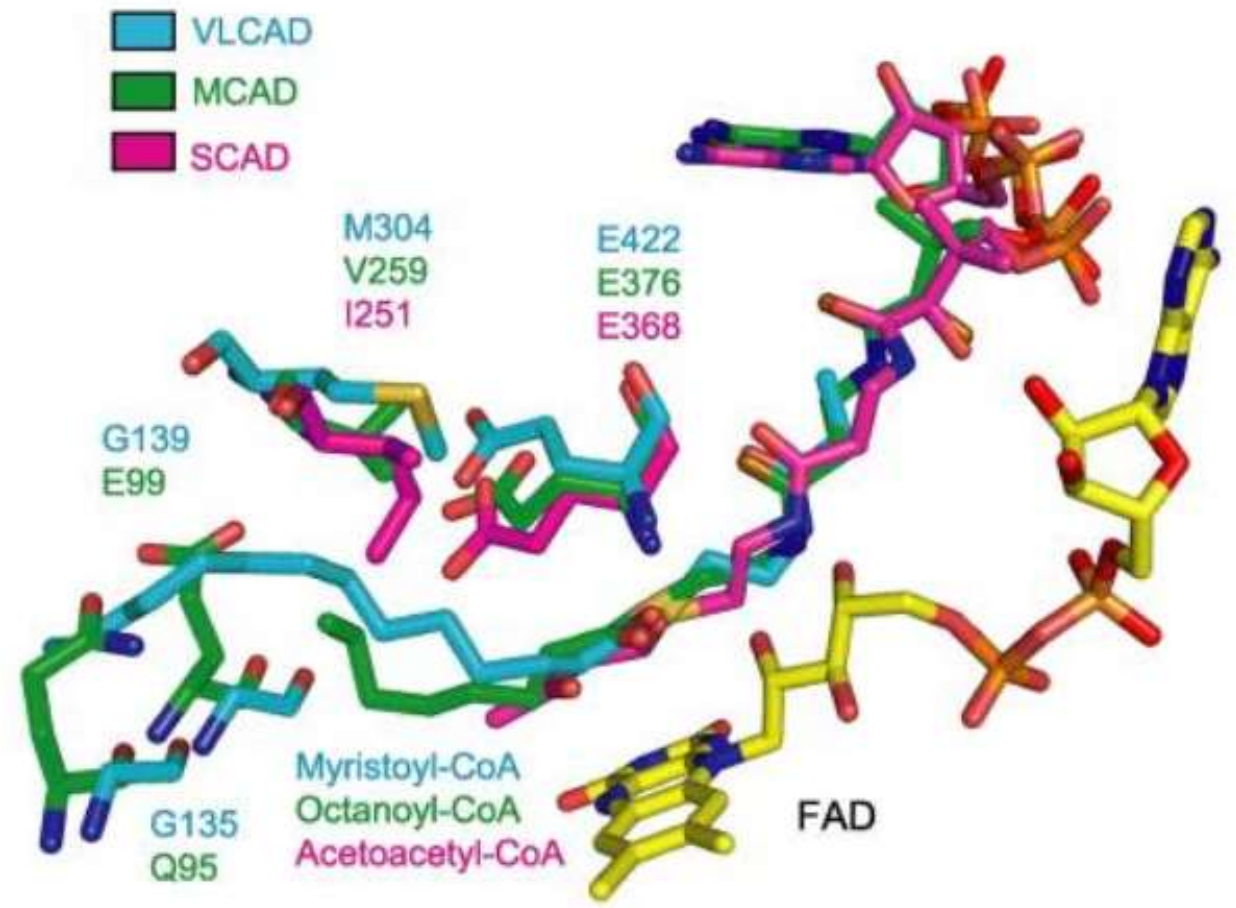

B

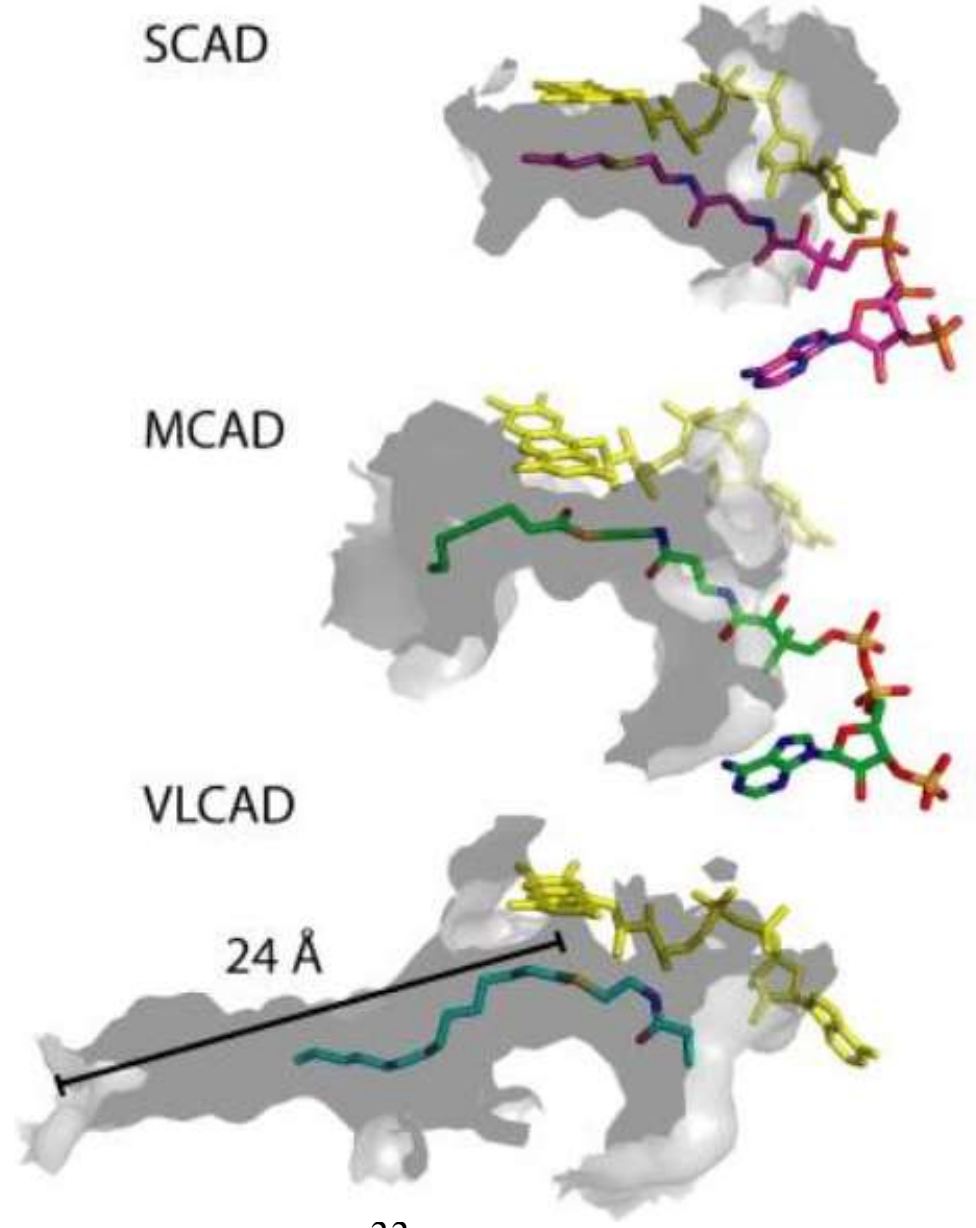


Figure 1.8.2 A catalytic glutamic acid residue initiates the ACAD reaction in the fat binding cavity

(A) The overlay highlights the conserved glutamic acid residue initiating the ACAD reaction in VLCAD (blue), MCAD (green), SCAD (magenta) for the fourteen, eight, and four carbon long fats. Critical residues that determine the depth and width of the binding cavity are also highlighted for VLCAD, MCAD, and SCAD. (B) Surface rendering demonstrating the depth of the binding cavities of SCAD (8 $\AA)$, MCAD (12 $\AA$ ), VLCAD $(24 \AA)$ with a fourteen, eight, and four carbon long bound fat. (A, B) reproduced from an open source article (McAndrew et al., 2008). Abbreviations: FAD: flavin adenine dinucleotide; SCAD: short chain acyl-CoA dehydrogenase; MCAD: medium chain acyl-CoA dehydrogenase; VLCAD: very long chain acyl-CoA dehydrogenase; E: glutamate; M: methionine; V: valine; I: isoleucine; G: glycine; Q: glutamine; Å: angstrom. 
Like VLCAD, MCAD and SCAD also utilize a glutamate as a catalytic residue, feature a non-covalently bound FAD cofactor, and recruit the ETF protein to shuttle elections to OXPHOS (Figure 1.8.2 A). The binding pockets in MCAD and SCAD are proportionally shorter to optimally accommodate fats 8 and 4 carbons long. VLCAD expresses the methionine (Met)-304 along and the glycine (Gly)-135 and Gly-139 residues at the base of the fat binding cavity (Figure 1.8.2 A), providing the widest and deepest cavity, compared to MCAD and SCAD, accommodating saturated fats up to 24 carbon atoms in length (Figure 1.8.2 B). The analogous residues are valine (Val)-259, glutamine (Gln)-95 and Glu-99 in MCAD and isoleucine (Ile)-251 in SCAD, resulting in less wide and shorter fat binding cavities, compared to VLCAD (Figure 1.8.2 A, B). These structural differences result in fat binding sites of varying volume, ensuring length specificity in the ACAD family of enzymes (Schiff et al., 2013).

\subsubsection{VLCAD Loss In Mice}

Currently, no widely available chemical VLCAD inhibitor exists. The cardiac-specific VLCAD knockout $\left(\mathrm{cVLCAD}^{-/}\right)$mouse showed no significant differences in fertility, long term survival, body fat mass, lean body mass, and body water, compared to wild type mice. In this cardiac specific VLCAD knockout model, FAO activity and overall mitochondrial respiration were decreased, compared to wildtype mice. Compared to the wildtype, cVLCAD ${ }^{-/}$mice demonstrated a decreased tolerance to cold exposure, prolonged fasting, and vigorous exercise. Murine cardiomyocytes with wild type VLCAD utilize FAO to generate up to $70 \%$ of their ATP content. ATP content in the $\mathrm{cVLCAD}^{-/}$myocytes were significantly decreased, compared to wild type counterparts. These separate metabolic stresses triggered a transient decrease in blood glucose levels from compensatory glucose metabolism, due to the inability to utilize fat, but did not result in murine death. These findings suggest that increased glucose oxidation may partially compensate 
for the loss of cardiac VLCAD and that transient metabolic stresses does not affect murine survival (Xiong et al., 2013).

VLCAD knockout in C57BL6 mice showed no change in long-term survival rates, compared to mice with wild type VLCAD, and demonstrated various compensatory mechanisms in different FAO-dependent tissues. For example, muscle cells switch to glucose oxidation by remodelling muscle into type II glycolytic fibres. In contrast, hepatocytes demonstrated no net increase in ACAD levels, compared to wildtype, but increased the contribution of long chain acylCoA dehydrogenase (LCAD), an isoform that has an intermediate and overlapping chain specificity with the VLCAD and MCAD enzymes, to FAO activity; combined LCAD and MCAD activity in hepatocytes lacking VLCAD prevented the accumulation of long chain fats such as C16 in mitochondria (Tucci et al., 2012).

Both studies critically show that loss of VLCAD activity in various tissues perturbs mitochondrial metabolism but is well tolerated and does not cause early death, compared to wild type mice (Xiong et al., 2013)(Tucci et al., 2012). However, while the LCAD gene is present in both humans and mice, LCAD is not expressed at the protein level and is not upregulated upon loss of VLCAD activity in humans (Houten \& Wanders, 2010). This critical difference between human and murine FAO underscores the importance of assessing VLCAD deficiency in humans to ensure loss of VLCAD is tolerable as a potential anti-AML therapeutic avenue.

\subsubsection{Clinical Human VLCAD Deficiency}

Human VLCAD deficiency is a rare metabolic disorder caused by a mutation adversely affecting VLCAD activity (Wanders et al., 2010). This inborn error of metabolism occurs at a rate between 1:30,000 - 1:100,000. At the molecular level, VLCAD mutations are highly heterogeneous with some mutations directly affecting reaction catalysis and others adversely 
affecting VLCAD mRNA stability. In a patient of VLCAD deficiency, single or multiple missense, single amino acid deletion, splice site abnormalities, and frame-shift mutations may be responsible for the loss of VLCAD activity. The loss of VLCAD activity is proportional to the severity of the resulting metabolic imbalance; VLCAD deficiency results in the accumulation of fatty acids, decreased FAO contribution to the TCA cycle, and an energy deficiency (Schiff et al., 2013). Adult patients with high residual VLCAD activity may be asymptomatic until exacerbation by vigorous physical activity, prolonged fasting, or consuming a meal high in long chain fats (Vockley et al., 2021). In patients with severe VLCAD deficiency, the three most prominent phenotypes manifest in cardiomyopathy, hypoketotic hypoglycemia, and rhadomyolysis in cardiac, hepatic, and muscle tissues, respectively. The cardiomyopathy results in cardiac arrythmia while the hypoglycemia triggers seizures and loss of consciousness. While there is currently no cure, these symptoms are managed with frequent meals, a diet low in long chain fats and high in medium chain fats, and avoiding prolonged, intense physical exercise. Dietary supplementation with medium chain fats has clinical benefit in managing cardiomyopathy and improving moderate exercise tolerance. The seven-carbon long fat sodium heptanoate is processed by medium chain FAO enzymes, which are unaffected by the FAO blockage at VLCAD, into acetyl-CoA and propionyl-CoA. Propionyl-CoA can be converted to succinyl-CoA, a TCA metabolite. Taken together, medium chain fat supplementation partially alleviates the metabolic consequences of VLCAD activity by restoring some supply of reducing equivalents to the ETC, as well as by providing acetyl-CoA and succinylCoA to the TCA cycle (Vockley et al., 2016).

Early detection of human fatty acid oxidation disorders such as VLCAD deficiency is determined by acyl-carnitine (AC) profiling by mass spectrometry (Okun et al., 2002) and VLCAD enzyme kinetic assays (Vockley et al., 2004). A rapid diagnosis is paramount for the survival of 
neonatal and pediatric patients. Loss of VLCAD activity causes an accumulation of VLCAD substrates, such as palmitate, a saturated 16-carbon long fat, and a depletion of medium and short chain FAO intermediates. To alleviate the build up of long chain acyl-CoA's, the mitochondria trans-esterifies the acyl-CoA substrates to AC's, allowing their export from the mitochondria into extracellular space, temporarily alleviating an intra-mitochondrial build up of acyl-CoA. These exported acyl-carnitines accumulate in peripheral blood and are detectable by mass spectrometry. Different FAO enzyme deficiencies exhibit specific accumulations and depletions of acylcarnitines; these characteristic AC profiles are the first step in the clinical diagnosis of an FAO disorder (Okun et al., 2002). As a follow-up to AC profiling, VLCAD kinetics is routinely assessed with a fluorescence-based assay using patient-derived fibroblasts; VLCAD activity is quantified with a fluorescence spectrophotometer via the quenching of fluorescence upon ETF reduction (Vockley et al., 2004).

Take together, AML is a devasting blood cancer with outdated, non-specific treatment options that fail to fully eliminate the leukemia population. Relapse initiating cells survive chemotherapy by becoming over-reliant on high rates of FAO, compared to normal cells, while exhibiting a diminished capacity to switch to alternative oxidative pathways. Compounds under preclinical consideration targeting CD36 and CPT1 have not been approved for anti-AML use in humans or trigger unacceptable off-target toxicities, preventing widespread clinical use. These drawbacks highlight the need to identify novel protein targets and preclinical chemical inhibitors to exploit FAO dependency and metabolic rigidity, both hallmark features of AML metabolism. 


\subsection{Chapter 2 Rationale And Hypotheses}

\subsection{Rationale}

Recent studies have highlighted the rationale and the pre-clinical potential of targeting cellsurface and mitochondrial-surface FAO enzymes in AML (Samudio et al., 2010)(German et al., 2016)(Shafat et al., 2017)(Zhang et al., 2020)(Wu et al., 2015). First, certain AML populations and cell lines are more reliant on higher rates of FAO, compared to the bulk hematopoietic population (Samudio et al., 2010)(German et al., 2016)(Shafat et al., 2017). Previous studies have also demonstrated that normal hematopoietic populations have an increased capacity to switch to compensatory metabolic pathways such as glycolysis-linked lactate production upon inhibition of mitochondrial oxidative metabolism (Molina et al., 2018)(Lagadinou et al., 2013)(Sharon et al., 2019). This metabolic vulnerability demonstrates a therapeutic window in which FAO inhibition induces death in the AML population, while sparing the healthy hematopoietic population. In addition, increased FAO provided a pro-survival advantage to chemotherapy resistant cells initiating relapse, the primary cause of AML patient death (Farge et al., 2017)(Stevens et al., 2020); these studies underscore the need for additional mechanistic studies of FAO in AML.

However, the most well-studied FAO chemical inhibitors, such as ETO (Holubarsch et al., 2007), SSO (Zhang et al., 2020)(Ye et al., 2016), BMS-0309403 (Shafat et al., 2017), that have demonstrated anti-AML activity in vitro or in vivo have not been approved for clinical use in AML (Figure 1.7.1). ETO previously demonstrated unacceptable cardio- and hepatoxicity in patients of chronic congestive heart failure and cardiac angina (Holubarsch et al., 2007). Other FAO inhibitors such as ranolazine and trimetazidine, both inhibitors of long chain 3-ketoacyl thiolase, required millimolar concentrations in vitro to inhibit AML proliferation (Samudio et al., 2010). There is also still debate over tissue specific mechanisms of action of clinical-grade FAO inhibitors - some 
studies demonstrated that the primary action of ranolazine in cardiomyocytes was endoplasmic reticulum calcium release (MacInnes et al., 2003) while others believe it to be FAO inhibition (Kantor et al., 2000). Taken together, specific targets such as CPT1 may be associated with cardioand hepatotoxicity. This suggests that certain mechanisms of action may be tissue specific, preventing widespread clinical use in AML (Holubarsch et al., 2007)(Fromenty \& Pessayre, 1995). Proposed anti-AML FAO inhibitors must demonstrate inhibition of FAO in the AML population while being well-tolerated by the normal hematopoietic system and other non-transformed cells.

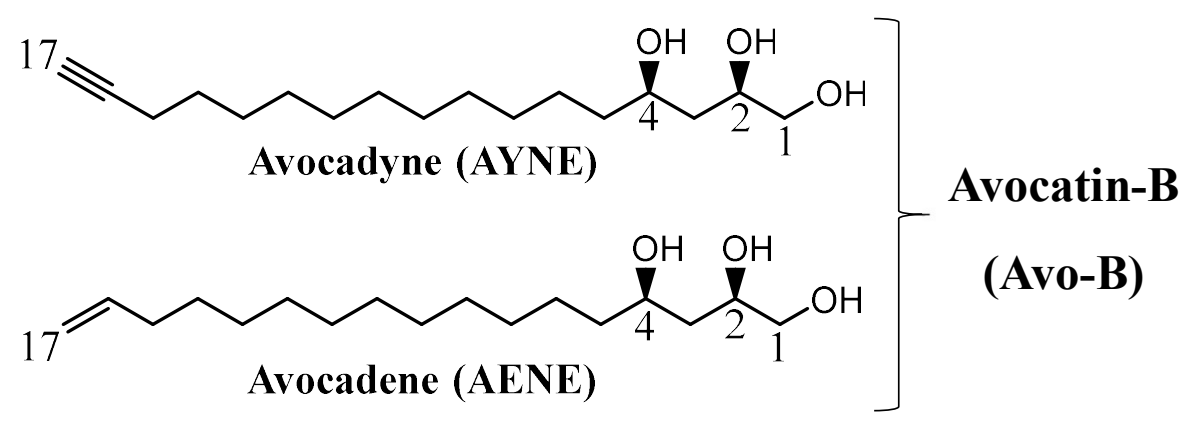

Figure 2.1.1 Structure Of The Constituents Of Avocatin-B (Avo-B)

This figure exhibits the structures of avocadyne (AYNE) and avocadene (AENE), which are both constituents of the avocado-derived mixture avocatin-B (avo-B) (Kashman et al., 1969)(Sugiyama et al., 1982). Drawn in ChemDraw 20.0.

Avocatin-B (avo-B) (See structure: Figure 2.1.1), a 1:1 molar ratio of the avocado-derived fatty alcohols avocadyne (AYNE) and avocadene (AENE), was cytotoxic towards AML cell lines and suppressed clonogenic growth and engraftment capacity of primary patient-derived cells, while sparing normal donor-derived hematopoietic cells in vitro and in vivo. Preceding AML death, this mixture localized to the mitochondria via CPT1 where it inhibited mitochondrial respiration supported by exogenous palmitate and increased oxidative stress by depleting endogenous antioxidants, such as NADPH and glutathione (GSH)(Lee et al., 2015). 
Avo-B also attenuated insulin resistance driven by abnormally high rates of FAO in vitro and in vivo. In lipotoxic murine $\mathrm{C} 2 \mathrm{C} 12$ muscle and rat INS-1 pancreatic $\beta$-islet cell lines, avo-B did not induce cell death but inhibited FAO and triggered increased glucose oxidation. This improved insulin response in murine muscle cells and initiated insulin secretion in rat pancreatic islet cells. While on a high fat diet to simulate diet induced obesity (DIO), mice receiving avo-B orally demonstrated an increased capacity to utilize glucose, as measured glucose and insulin tolerance tests. Plasma free fatty acid levels as well as PDH activity were restored in mice receiving avo-B on the high fat diet to the levels observed in mice on a standard diet, confirming restoration of insulin signalling in vivo. A phase 1 pilot human clinical trial demonstrated that a supplement containing $200 \mathrm{mg}$ avo-B daily for 60 days was well tolerated with no limiting toxicities (Ahmed et al., 2019). Despite demonstrating a potential therapeutic window for anti-AML use and being well-tolerated in a pilot clinical trial, additional experiments with avo-B are required to show antiAML efficacy.

\subsection{Hypotheses}

\section{Demonstrate specificity of AML cytotoxicity by avo-B and identify the active anti-AML component of avo-B}

Combinations of compounds may interact synergistically, antagonistically, or in an additive manner to elicit therapeutic effects (Chou, 2010). In chapter 4, the hypothesis will be tested that one of the fatty alcohols of avo-B, when given as a single agent, is more potent than the mixture, in terms of cytotoxicity against immortalized and primary AML cells.

To evaluate this hypothesis, the objectives of chapter 4 were to: 
1. Determine the specificity of avo-B cytotoxicity on myeloid leukemia, solid tumor, multiple myeloma, and non-transformed cell lines

2. Assess the anti-AML activity of avo-B in combination with frontline AML therapeutics ara-C and doxorubicin (DOX) using Calcusyn

3. Assess the anti-AML activity of the two constituents of avo-B as single agents and as a combination using Calcusyn

\section{Elucidate the mechanism of action and molecular target of the active anti-AML component of avo-B}

Avo-B was previously shown to accumulate in the mitochondria to suppress fat-supported respiration (Lee et al., 2015). In addition, inhibition of FAO was confirmed in C2C12 and INS-1 cell lines (Ahmed et al., 2019). In chapter 5, the hypothesis will be tested that the molecular target of this active component will be an intramitochondrial protein involved in long chain FAO.

To evaluate this hypothesis, the objectives of chapter 5 were to:

1. Assess gene and protein expression of the four intramitochondrial enzymes catalyzing long chain FAO in AML and normal hematopoietic cells.

2. Determine the role of a novel overexpressed intramitochondrial FAO enzyme in mitochondrial metabolism as well as AML proliferation, clonogenic growth, and engraftment capacity.

3. Conduct pharmacodynamic studies to assess the interaction between the overexpressed intramitochondrial FAO enzyme and AYNE with liquid scintillation counting, kinetic assays, respirometry, acyl-carnitine profiling, stable isotope tracers. 
4. Assess mitochondrial oxidative metabolism of both AML and normal hematopoietic cells treated with AYNE using stable isotope tracers.

5. Assess in vivo efficacy of AYNE with NSG xenograft studies.

\section{Determine the role of structural moieties and stereochemistry of avo-B's components on its ability to inhibit FAO}

The two components of avo-B are structurally similar; both are 17 carbons in length, trihydroxylated, and differ in terminal unsaturation. In chapter 6 , the hypothesis will be tested that a structure-activity relationship exists where the odd number carbon length, the terminal unsaturation, and the stereochemistry of its chiral carbon atoms are critical to avo-B's ability to inhibit FAO.

To evaluate this hypothesis, the objectives of chapter 6 were to investigate the role of the abovementioned structural groups in the constituents of avo-B, with a focus on AYNE, to:

1. Induce AML death in vitro and in vivo

2. Bind to VLCAD, inhibit FAO as well as fat-supported mitochondrial respiration 


\subsection{Chapter 3 Materials And Methods}

\subsection{Cell Culture}

TLS-ERG immortalized (TEX) myeloid leukemia cells were a generous gift from the group of Prof. Dick (University Health Network) were maintained in Iscove's modified Dulbecco's medium (IMDM; \#319-105-CL; Wisent), 15\% fetal bovine serum (FBS; \# 080-150; Wisent), 2mM 1glutamine (G7513; Sigma), 2\% penicillin-streptomycin (450-200-EL; Wisent), $20 \mathrm{ng} / \mathrm{mL}$ stem cell factor (SCF; \#300-07; Peprotech), 2 ng/mL interleukin-3 (IL-3; \#200-3; Peprotech)(Pereira et al., 1998)(Warner et al., 2005). Ontario Cancer Institute AML2 (AML2) myeloid leukemia cells, Jurkat T cell lymphoma cells, U937 myeloid lymphoid cells, HL-60 promyelocytic leukemia cells, and KG1a promyelocytic leukemia cells were maintained in IMDM, 10\% FBS, 2\% penicillinstreptomycin. Multiple myeloid cell lines (LP-1, MMS1-1, RPMI 8226, RPMI 4921) were cultured in Roswell Park Memorial Institute medium 1640 (RPMI; R0883; Sigma), 10\% FBS, 2\% penicillin-streptomycin. The media of Jurkat $\rho 0$ cells was supplemented with $50 \mu \mathrm{g} / \mathrm{mL}$ uridine (U3003; Sigma) and $1 \mu \mathrm{g} / \mathrm{mL}$ ethidium bromide (E1510; Sigma). Medical Research Council cell strain-7 (MRC-7) human fetal lung, human embryonic kidney 293 (HEK293), HeLa human cervical cancer, HCT116 human colorectal cancer, MDA-MB-486 human breast cancer, and HT1080 fibrosarcoma cells were maintained in Dulbecco's modified eagle medium (DMEM; 319016-CL; Wisent), 10\% FBS, 2\% penicillin-streptomycin; these adherent lines were passaged with 0.25\% trypsin-ethylenediaminetetraacetic acid (EDTA; \#324506; Sigma). All immortalized cell lines were cultured in $5 \% \mathrm{CO}_{2}$ at $37^{\circ} \mathrm{C}$ and passaged up to 25 times, as done previously (Spagnuolo et al., 2013).

Human AML and normal samples were provided by the groups of Prof. Hess (Western University), Prof. Hope (McMaster University), Prof. Minden (Princess Margaret Hospital) or 
Stem Cell Technologies (Cat\#70025). Primary AML cells were isolated from peripheral blood of consenting AML patients, who had at least $80 \%$ malignant cells upon sample collection. Cells were isolated by Ficoll Type 400 density gradient centrifugation (F5415; Sigma). Normal mononuclear cells (MNC) from peripheral blood were obtained from consenting donors without AML after granulocyte-colony stimulating factor mobilization. The collection and use of human tissue was approved by institutional review at either Western University, McMcaster University, or the University Health Network. CD34+ AML and MNC cells were isolated from primary samples by immunomagnetic positive isolation via the EasySep ${ }^{\mathrm{TM}}$ Human CD34 Positive Selection Kit (Cat\# 17856; Stem Cell Technologies) and confirmed via flow cytometry with an anti-human CD34 antibody (Cat\# 6797; BioVision).

For metabolic tracer experiments, primary cells were thaw and enumerated in IMDM supplemented with $10 \%$ fetal bovine serum (FBS), $2 \%$ penicillin-streptomycin, prior to resuspension into media containing a tracer. AML patient cytogenetic profiles are tabulated at the end of chapters 4,5 , and 6 with the reporting style as follows: 1 . overall number of chromosomes identified, 2. sex chromosomes, 3. affected chromosomes, 4. type of abnormalities described in short hand, 5. chromosomal band location.

\subsection{Source Of Avocatin-B (Avo-B), Avocadyne (AYNE), Avocadene (AENE)}

For experiments in chapters 4 and 5, Avo-B, avocadyne (AYNE; CAS\#129099-96-3), avocadene (AENE; CAS\# 83797-45-9) were provided by MicroSource Discovery (Gaylordsville, CT, USA). For experiments in chapter 6, the synthetic stereoisomers of AYNE and AENE were a generous gift from the group of Prof. Lewary (University of Alberta)(Cunha et al., 2019). Regardless of source, avo-B, AYNE, and AENE were solubilized in dimethyl sulfoxide (DMSO; 
D2650; Sigma). For rescue experiments, sodium heptanoate (E0009; TCI America) was solubilized in phosphate buffered saline (PBS; Cat\# 10010023; Gibco).

\subsection{Cell Growth And Viability}

Cell viability, and clonogenic growth were carried out as previously described using either the 3-(4,5-dimethylthiazol-2-yl)-5-(3-carboxymethoxyphenyl)-2-(4-sulfophenyl)-2H-tetrazolium inner salt (MTS; G3582; Promega) dye and a UV-Vis spectrophotometer (Synergy ${ }^{\mathrm{TM}} 2$ Multimode Microplate Reader; Biotek) or the dye 7-aminoactinomycin (7-AAD; Cat\# 2725; BioVision) on a flow cytometer (Guava EasyCyte 8HT; EMD Millipore) (Spagnuolo et al., 2013). For the MTS protocol, $20 \mu \mathrm{L}$ of the MTS reagent was added to $100 \mu \mathrm{L}$ of cell media in a well of a 96 well plate (Cat\#167800; Nunclon). Cells were incubated at $37^{\circ} \mathrm{C}$ for two hours and absorbance was measured at $490 \mathrm{~nm}$. For the 7AAD protocol, cells were washed once with PBS and incubated in $100 \mu \mathrm{L}$ of $50 \mu \mathrm{g} / \mathrm{mL}$ of $7 \mathrm{AAD}$ solvated in PBS for 30 minutes at $37^{\circ} \mathrm{C}$. Cells were washed and re-suspended in PBS and analyzed on the flow cytometer. To assess cell growth and proliferation, $50 \mu \mathrm{L}$ of cells in growth media were mixed with $50 \mu \mathrm{L}$ of $0.4 \%$ trypan blue (Cat\#15250061; Sigma). $10 \mu \mathrm{L}$ of this mixture was loaded onto a hemocytometer for enumeration.

\subsection{Colony Formation Assay}

As assessed previously (Spagnuolo et al., 2013), clonogenic growth of TEX, AML2 primary AML, normal MNC cells, $1 * 10^{5}$ cells/ $\mathrm{mL}$ in duplicate onto $35 \mathrm{~mm}$ dishes (Cat\#150460; Nunclon) in MethoCult GF H4434 semisolid media (Cat\#04434; Stem Cell Technologies), containing 1\% methylcellulose in IMDM, 30\% FBS, 1\% bovine serum albumin, $3 \mathrm{U} / \mathrm{ml}$ recombinant human erythropoietin, $10^{-4}$ M 2-mercaptoethanol, 2mM L-glutamine, 50ng/ml recombinant human stem cell factor, $10 \mathrm{ng} / \mathrm{ml}$ recombinant human granulocyte macrophage-colony stimulating factor and 
$10 \mathrm{ng} / \mathrm{ml}$ recombinant human IL-3. Colonies (a cluster of 10 or more cells) were enumerated following incubation at $37^{\circ} \mathrm{C}$ and $5 \% \mathrm{CO}_{2}$ for 14 days.

\subsection{Database Profiling}

Datasets (GSE9476, GSE13204, GSE1159) and probe lists for gene expression platforms were downloaded from https://www.ncbi.nlm.nih.gov/geo/, separated into two groups: normal healthy bone marrow (BM) and AML patient groups, and compared with a two-tailed, unpaired Student's $t$-test in GraphPad Prism 7.0. All non-AML patient samples were excluded from the analysis.

\subsection{Co-Immunoprecipitation Experiments}

Co-immunoprecipitation (co-IP) of VLCAD was carried using the Pierce Crosslink Magnetic Co-IP Kit (Cat\#88805; ThermoFisher Scientific) with the following antibodies: human antiVLCAD for co-IP (Cat\#14527-1-AP; ProteinTech). Briefly, AML2 (10*10^6 cells) were treated with $10 \mu \mathrm{M}$ of avocadyne in growth media for three hours at $37^{\circ} \mathrm{C}, 5 \% \mathrm{CO}_{2}$. During this incubation step, $5 \mu \mathrm{g}$ of anti-VLCAD antibody (ProteinTech) was coupled to $25 \mu \mathrm{L}$ of magnetic beads with $2 \mathrm{mg}$ of kit provided disuccinmidyl suberate acting as a coupling agent. Cells were then lysed with radioimmunoprecipitation assay (RIPA) buffer (R0278; Sigma) on ice. The cell lysate was then exposed to the anti-VLCAD antibody coupled to the magnetic beads overnight at $4{ }^{\circ} \mathrm{C}$ with gentle agitation with a lab gyrator. The lysate/antibody coupled to magnetic beads mixture were separated with a DynaMagTM 2 Magnet (Cat\#12321D; Pierce). Following the overnight incubation, the lysate/magnetic bead mixture was applied to the magnet for 5 minutes at $24^{\circ} \mathrm{C}$. At this point, the AYNE-VLCAD complex was bound to the antibody-beads; the lysate (containing all protein except VLCAD) is carefully pipette out to produce the flow through fraction. The magnetic beads coupled to the VLCAD antibody and VLCAD protein are washed twice with $1 \mathrm{~mL}$ of the kit provided immunoprecipitation wash buffer. The VLCAD protein is then eluted from the beads 
with $100 \mu \mathrm{L}$ of a kit provided low $\mathrm{pH}$ elution buffer for 10 minutes with gentle agitation at $24^{\circ} \mathrm{C}$ on a lab gyrator. The $100 \mu \mathrm{L}$ fraction is the VLCAD-enriched fraction, contains exclusively VLCAD protein, and immediately used in western blot analysis or frozen at $-82{ }^{\circ} \mathrm{C}$ for subsequent quantification of AYNE using our previously published method (Ahmed et al., 2018).

\subsection{Immunoblotting}

Western blotting, consisting of lysate preparation, protein quantification, and sodium dodecyl sulfate polyacrylamide gel electrophoresis (SDS-PAGE), was conducted as previous described (Spagnuolo et al., 2013).

Cell lysate preparation was conducted with reagents chilled on ice. Cells were washed once in PBS; a 1:1 volume of radioimmunoprecipitation assay (RIPA; Sigma) buffer supplemented with protease inhibitor (Cat\#04693116001; Sigma) for 20 minutes with frequent vortexing. Cells were then centrifuged at $13,200 \mathrm{rpm}$ for 20 minutes at $4^{\circ} \mathrm{C}$. The supernatant was carefully collected in new centrifuge tubes and stored on ice for protein quantification.

Total protein quantification was determined using the bicinchoninic acid (BCA) protein assay. A standard curve was produced with BSA (Cat\#10775835001; Sigma) solvated in distilled water (Cat\#15230071; Gibco) at concentration range from $0-1.0 \mathrm{mg} / \mathrm{mL}$. Lysates, diluted tenfold, and protein standards were plated in triplicate on a 96 well plate. To each well, $200 \mu \mathrm{L}$ of BCA working reagent (B9643; Sigma)(50 parts: 1 part copper II sulfate (C2284; Sigma)) was added to each well. The plate was incubated at $37^{\circ} \mathrm{C}$ for 30 minutes and absorbance was read at $527 \mathrm{~nm}$ using a UV-Vis spectrophotometer. The undiluted protein samples were diluted in 3 parts RIPA and 1 part sample buffer (240 mM Tris- $\mathrm{HCl}(\mathrm{Cat} \# 10812846001$; Sigma $)$ at $\mathrm{pH} 6.8,6 \% \mathrm{w} / \mathrm{v}$ Sodium dodecyl sulfate (SDS; L3771; Sigma), 30\% v/v sucrose (S0389; Sigma), 0.02\% w/v bromophenol blue (B0126; Sigma), and $50 \mathrm{mM}$ dithiothreitol (Cat\#43815; Sigma)) to $1 \mu \mathrm{g} / \mathrm{mL}$. 
Samples diluted in sample buffer were either stored at $-82^{\circ} \mathrm{C}$ till further use or underwent SDSPAGE immediately.

Proteins were separated via SDS-PAGE on a 10-15\% separating gel in electrophoresis buffer (25 mM Tris base (Cat\#10708976001, Sigma), 190 mM glycine (G7126; Sigma), 3.5 mM SDS) mounted in a Mini Trans-Blot Cell (Cat\#1703930; Bio-Rad). Semi-dry transfer of proteins onto a PVDF membrane (Cat\#1620177; Bio-Rad) in transfer buffer (25 mM Tris base, $190 \mathrm{mM}$ glycine, 20\% v/v methanol (Cat\#322415; Sigma)) was conducted with a Trans-Blot semi-dry transfer cell (Cat\#1703940; Bio-Rad). Membranes were then blocked with 5\% BSA in Trisbuffered saline $\left(20 \mathrm{mM}\right.$ Tris base (TBS) plus $0.1 \%$ Tween (TBS-T) for one hour at $24^{\circ} \mathrm{C}$ to minimize non-specific binding. Blots were then incubated with primary antibody (see below table) solvated in TBS-T supplemented with $5 \%$ BSA overnight at $4{ }^{\circ} \mathrm{C}$ with gentle agitation on a lab gyrator. Membranes were washed three times with TBS-T, and then incubated with appropriate secondary antibody (Cell Signalling Technologies; CST) solvated in TBS-T for one hour at $24^{\circ} \mathrm{C}$. Antibodies used in immunoblotting are tabulated in Table 3.7.1. Membranes were washed three additional times with TBS-T. Western enhanced chemiluminescent substrate (Cat\#1705061; BioRad) was used to visualize proteins on a SynGene ProImager Station.

Table 3.7.1 Western Blot Antibodies

\begin{tabular}{|c|c|c|c|}
\hline Antibody Target & Isotype & $\begin{array}{c}\text { Molecular Weight } \\
\text { (kDa) }\end{array}$ & Supplier \\
\hline Human VLCAD & Rabbit & 68 & $\begin{array}{c}\text { (HPA020595; } \\
\text { Sigma) }\end{array}$ \\
\hline Human GAPDH & Mouse & 36 & $(\# 2118 ;$ CST) \\
\hline Human $\beta$-actin & Rabbit & 42 & $(\# 4967 ;$ CST) \\
\hline
\end{tabular}




\subsection{High Resolution Respirometry}

Oxygen flux was assess using the high resolution respirometer Oxygraph-O2k (Oroboros Instruments). The respirometer performed an oxygen curve calibration using sodium dithionite (Cat\#1.06505; Sigma) once every three months. For intact cell respiration, respirometer chambers were filled with PBS; for permeabilized cell respiration, respirometer chambers were filled with mitochondrial respiration buffer 05 (MiR05) with all components from Sigma (Ojuka et al., 2016). The formulation is tabulated in Table 3.8.1. The components listed below were solvated in distilled water and $\mathrm{pH}$ corrected $\mathrm{pH}$ to 7.5 with potassium hydroxide (KOH; Cat\#221473; Sigma). For permeabilization protocols, a 10\% digitonin (Cat\#260746; Sigma) solution is solvated in DMSO and stored at $-20^{\circ} \mathrm{C}$ until use. On the day of use, dilute the $10 \%$ solution to $1 \%$ in PBS.

Table 3.8.1 Respiration Buffer MiR05 Formulation

\begin{tabular}{|c|c|c|}
\hline \multicolumn{3}{|c|}{ MiR05 Formulation } \\
\hline Compound & Molecular Weight (g/mol) & Final Concentration (mM) \\
\hline EGTA (\#324626; Sigma) & 380.4 & 0.5 \\
\hline $\mathrm{MgCl}_{2}$ (M1028; Sigma) & 92.5 & 3 \\
\hline $\begin{array}{c}\text { Lactobionic acid (\#153516; } \\
\text { Sigma) }\end{array}$ & 358.3 & 60 \\
\hline $\begin{array}{c}\text { Taurine (T0625; Sigma) } \\
\mathrm{KH}_{2} \mathrm{PO}_{4} \text { (P0622; Sigma) }\end{array}$ & 125.1 & 20 \\
\hline $\mathrm{HEPES} \mathrm{(H4034;} \mathrm{Sigma)}^{\text {D-sucrose (S0389; Sigma) }}$ & 136.1 & 10 \\
\hline $\begin{array}{c}\text { BSA (fatty acid free) (A8806; } \\
\text { Sigma) }\end{array}$ & 238.3 & 110 \\
\hline
\end{tabular}

To assess intact cell respiration (Karabatsiakis et al., 2014), $5^{*} 10^{6}$ cells were solvated ed in PBS with a compound of interest as well as FAO substrates ( $200 \mu \mathrm{M}$ sodium palmitate (P9767; 
Sigma) coupled to BSA (Fatty acid free) and $1 \mathrm{mM} \mathrm{L-carnitine} \mathrm{(C0283;} \mathrm{Sigma)} \mathrm{for} 1 \mathrm{hr}$, washed once in PBS, and injected intact into the respirometer. Cells demonstrate a basal level of respiration. In sequence, $250 \mathrm{nM}$ oligomycin (OLI; \#75351; Sigma), $500 \mathrm{nM}$ the uncoupler carbonyl cyanide-p-trifluoromethoxyphenylhydrazone (FCCP; C2920; Sigma) and $1 \mathrm{mM}$ sodium pyruvate (PYR; P2580; Sigma) were injected; this results in respiration uncoupled from OXPHOS and maximal respiration, respectively. $250 \mathrm{nM}$ antimycin A (ANTIA; A8674; Sigma) results in all non-mitochondrial respiration.

To assess fat supported ETF respiration in permeabilized cells (Ojuka et al., 2016), cells were treated for one hour with a compound of interest, washed once in PBS, permeabilized in 1\% digitonin for 3 minutes. The pellet was re-suspended in PBS, and was injected into the respirometer. To stimulate fat supported respiration, either $25 \mu \mathrm{M}$ of palmitoyl-carnitine (PC; P1645; Sigma) or octanoyl-carnitine (OC; \#50892; Sigma) as well as $2 \mathrm{mM}$ malate (MAL; \#1613881; Sigma), 2.5 mM adenosine diphosphate (ADP; A2754; Sigma) were added. $250 \mathrm{nM}$ rotenone (ROT; R8875; Sigma) and 5 mM malonate (MALON; M1296; Sigma) are then added to inhibit CI and CII. $250 \mathrm{nM}$ ANTIA results in all non-mitochondrial respiration.

To assess complex I and II respiration in permeabilized cells (Makrecka-Kuka et al., 2015), cells were treated with a compound of interest for one hour, permeabilized in $1 \%$ digitonin for three minutes, and the pellet was injected into the respirometer. To stimulate CI-supported respiration, 5 mM PYR, 10 mM monosodium glutamate (MSG; G8415; Sigma), 2 mM MAL, 2.5 mM ADP are injected. $250 \mathrm{nM}$ ROT inhibits CI respiration. To stimulate CII-supported respiration, $10 \mathrm{mM}$ succinate (S3674; Sigma) is added. $250 \mathrm{nM}$ ANTIA results in all nonmitochondrial respiration. 


\subsection{Fatty Acid Oxidation By Liquid Scintillation Counting}

To directly quantify FAO activity through the release of ${ }^{14} \mathrm{CO}_{2}$ (Huynh et al., 2014), sodium palmitate was esterified to fatty-acid free BSA to a final concentration of $7.5 \% \mathrm{BSA} / 5 \mathrm{mM}$ palmitate (PA-BSA) solvated in distilled water. $16-{ }^{14} \mathrm{C}$ palmitate (NEC534050UC; Perkin Elmer) was integrated into the PA-BSA at $37^{\circ} \mathrm{C}$ with frequent vortexing for one hour. The radio-labelled PA-BSA mixture is incubated at $37^{\circ} \mathrm{C}$ overnight, prior to addition to the cells of interest.

Cells $\left(0.5^{*} 10^{\wedge} 6\right.$ cells $\left./ \mathrm{mL}\right)$ were treated with compounds of interest and exposed to a final concentration of $0.3 \% \mathrm{BSA} / 100 \mu \mathrm{M}$ palmitate $/ 0.4 \mu \mathrm{Ci} / \mathrm{mL}{ }^{14} \mathrm{C}$-palmitate and $1 \mathrm{mM}$ 1-carnitine. $\mathrm{CO}_{2}$ capture tubes were created by placing a filter paper with a pore size of 20-25 micron (WHA1441090; Whatman) soaked in $1 \mathrm{M} \mathrm{NaOH}$ in the cap of the tube. After three hours at $37^{\circ} \mathrm{C}$ and $5^{\circ} \mathrm{C}$, cells were added to the $1 \mathrm{M}$ perchloric acid (\#244252; Sigma) in the bottom of a $\mathrm{CO}_{2}$ capture tube and gently agitated on an orbital shaker for $1 \mathrm{hr}$ at room temperature. The filter paper securing the released ${ }^{14} \mathrm{CO}_{2}$ is then transferred to a liquid scintillation vial (Cat\#6000488; Perkin Elmer). Hyamine Hydroxide®10-X scintillation fluid (Cat\#6003005; Perkin Elmer) was added, and disintegrations per minute were read on a TRI-CARB 4910TR 110V Liquid Scintillation Counter (Perkin Elmer).

\subsection{Acyl-CoA Dehydrogenase (ACAD) Activity Assay}

To directly quantify ACAD activity (Vockley et al., 2004), the reaction mixture (50 mM Tris ( $\mathrm{pH} 8), 0.5 \%(\mathrm{w} / \mathrm{v})$ D-glucose, and $50 \mu \mathrm{M}$ acyl-CoA substrate (either palmitoyl- (C16-CoA; P9716; Sigma) or octanoyl-CoA (C8-CoA; O6877; Sigma)) is deaerated and layered with oxygen-

free argon in a tightly sealed quartz semi-microcuvette. 50 units of glucose oxidase (G7141; Sigma) and 1 unit of catalase (C9322; Sigma) eliminates remaining oxygen. $1 \mu \mathrm{M}$ purified recombinant porcine electron transfer flavoprotein $(\mathrm{ETF})$ and the protein sample of interest is then 
added to the reaction mixture. Specific activity is assessed via excitation of the ETF flavin at 342 $\mathrm{nm}$ and the quenching of fluorescence at $496 \mathrm{~nm}$, detected by an LS 50 Bioluminescence spectrometer (Perkin Elmer) set to $32^{\circ} \mathrm{C}$. The slope of the line is calculated with linear regression analysis; 1 unit of activity defined as the amount of enzyme required to reduce $1 \mu \mathrm{mol}$ of ETF per minute.

\subsection{Short Hairpin (shRNA) RNA Knockdown}

Lentiviral transductions were performed as described (Spagnuolo et al., 2013). For genetic knockdown of VLCAD, E. coli carrying a shRNA sequence targeting VLCAD inserted into pLKO.1 plasmids (See Table 3.11.1; GE Darmacon) were cultured in lysogeny broth (L3522; Sigma) in an orbital shaker at $37^{\circ} \mathrm{C}$. Plasmids were recovered via plasmid extraction kit (L210002; Qiagen) and quantified via Nanodrop ${ }^{\mathrm{TM}} 2000$ (Thermofisher). A third generation plasmid system was used in a 1:0.75:0.25 ratio of the lentiviral transfer plasmid carrying the shRNA sequence (pLKO.1): packaging plasmid (psPAX2): enveloping plasmid (pMD2.G). HEK 293T packaging cells were transfected with plasmid DNA and TransIT (Cat\#5406; Mirius Bio) solvated in Optimem media (Cat\#31985062; Gibco). TEX and AML2 cells were infected with $7.25 \mu \mathrm{g} / \mathrm{mL}$ protamine sulfate (P3369; Sigma) and the media containing lentivirus, followed by 7 days of $1 \mu \mathrm{g} /$ mL puromycin (P9620; Sigma) selection. Knockdown was confirmed with immunoblotting. The plasmid pLKO.1 - TRC cloning vector was a gift from David Root (Addgene plasmid \# 10878)(Moffat et al., 2006). The plasmid pMD2.G was a gift from Didier Trono (Addgene plasmid \# 12259). The plasmid psPAX2 was a gift from Didier Trono (Addgene plasmid \# 12260). 
Table 3.11.1 Anti-VLCAD Short Hairpin RNA Sequences

\begin{tabular}{|c|c|c|}
\hline \multicolumn{2}{|c|}{ VLCAD targeting shRNAs in the pLKO.1 vector (GE Darmacon) } \\
\hline $\begin{array}{c}\text { Manuscript } \\
\text { construct }\end{array}$ & Clone ID & $\begin{array}{c}\text { Mature Antisense Sequence } \\
\left(\mathbf{5}^{\prime}-\mathbf{3}^{\prime}\right)\end{array}$ \\
\hline D5 & TRCN0000028493 & AAGGACTTAGATTCCGCCTTG \\
\hline D6 & TRCN0000028559 & AAACTGGGTACGATTAGTGGC \\
\hline D7 & TRCN0000028554 & AAAGACCGTGAAGATGTCTGC \\
\hline D8 & TRCN0000028555 & ATGGACTCAGTTACATACTGC \\
\hline D9 & TRCN0000028515 & TACTCCATCAAAGAACACCTC \\
\hline
\end{tabular}

\subsection{Acylcarnitine Profiling}

To assess levels of FAO intermediate AC's (Okun et al., 2002), 1.5*10^5 cells $/ \mathrm{mL}$ nontransformed fibroblasts were maintained in Dulbecco's Modified Essential Media (DMEM; 319016-CL; Wisent) supplemented with $10 \%$ fetal calf serum (FCS; 075-250; Wisent) and $1 \%$ penicillin-streptomycin and passaged with $0.24 \%$ trypsin-EDTA (T4049; Sigma). 24 hours prior to the start of FAO assessment, fibroblasts were plated in HAM F-12 (Cat\#31765035; Invitrogen) supplemented with $2 \mathrm{mmol} / \mathrm{L}$ 1-glutamine, 10\% FCS, and 1\% penicillin-streptomycin. After 24 hours, fibroblasts were washed with PBS and provided with minimum essential medium (MEM; M154500; Invitrogen) supplemented with $200 \mu \mathrm{M}$ PA-BSA, $400 \mu \mathrm{M}$ L-carnitine, 2 mmol/L Lglutamine, 10\% FCS, and 1\% penicillin-streptomycin. Fibroblasts were treated with compounds of interest for 96 hours and the spent media was analyzed via triple quadruple MS-MS as previously described (Okun et al., 2002). The collection and use of human tissue was approved by institutional review at the University of Pittsburgh Medical Center Rangos Research Center (USA). 


\subsection{ATP, PDH (Pyruvate Dehydrogenase) Activity, Mitochondrial Mass Quantification}

ATP levels (MAK190; Sigma) and PDH activity (MAK183; Sigma) were quantified, according to kit manufacturer's instructions with no deviations. For mitochondrial mass quantification, mitotracker dye (M7514; Thermofisher) was solubilized in DMSO to a stock concentration of $20 \mathrm{mM}$. Cells were washed once in PBS, stained in PBS containing a final mitotracker dye concentration of $200 \mathrm{nM}$ for 45 minutes, re-suspended in PBS for flow cytometric analysis.

\subsection{Ultra High Performance Liquid chromatography (UHPLC) - Mass Spectrometry (MS) Analysis Of FAO, Glycolysis, And TCA Metabolites}

TEX, AML2, primary AML, or primary MNC $\left(2.5^{*} 10^{5}\right.$ cells $\left./ \mathrm{mL}\right)$ were treated with a compound of interest or solvent vehicle and co-incubated with uniformly labelled stable carbon $13\left({ }^{13} \mathrm{C}\right)$ palmitate $\left({ }^{13} \mathrm{C}_{16}\right.$; Cat $\# 409$; Cambridge Isotopes $)$ or glucose $\left({ }^{13} \mathrm{C}_{6}\right.$; Car\#1396; Cambridge Isotopes) for $12 \mathrm{hrs}$ in $\alpha$ MEM media (Cat\#310-010-CL; Wisent) supplemented with 5\% dialyzed FBS (Cat\#080-910; Wisent), 1\% pen-strep, $400 \mu \mathrm{M}$ L-carnitine, $650 \mu \mathrm{M}$ L-glutamine, and 100 $\mu \mathrm{M}$ L-glutamate (Jones et al., 2018). For experiments with $200 \mu \mathrm{M}{ }^{13} \mathrm{C}_{16}$ palmitate, this media was supplemented with $5.5 \mathrm{mM}$ D-glucose. For experiments with $5 \mathrm{mM}{ }^{13} \mathrm{C}_{6}$ glucose, this media was supplemented with $200 \mu \mathrm{M}$ PA-BSA. After 12 hours, cells were then collected, counted, washed three times in PBS, and frozen at $-82{ }^{\circ} \mathrm{C}$ until UHPLC analysis. Cells were then extracted in methanol:acetonitrile (Cat\#270717; Sigma):water (Cat\#270733; Sigma) (5:3:2 v/v/v) at a concentration of $1 * 10^{6}$ cells per $\mathrm{mL}$ of the 5:3:2 solvent, prior to UHPLC-MS analyses (VanquishQExactive, Thermo Fisher)(Jones et al., 2018).

At $4^{\circ} \mathrm{C}$, samples were vortexed for $30 \mathrm{~min}$ at $4^{\circ} \mathrm{C}$ and centrifuged for $10 \mathrm{~min}$ at $15,000 * \mathrm{~g}$. Ten $\mu \mathrm{L}$ of sample extracts were then injected onto a KinetexXB-C18 column $(150 \times 2.1 \mathrm{~mm}$ i.d., 
1.7mm; Phenomenex)(Jones et al., 2018). The chromatographic elution was achieved with a 3 min isocratic run (5\% B) and a 9 min gradient from 5-95\% B (phase A:water and B: acetonitrile with $0.1 \%$ formic acid (Cat\#1.59013; Sigma) or with $10 \mathrm{mM}$ ammonium acetate (Cat\#73594; Sigma) for positive or negative ion mode, respectively) to separate metabolites (Jones et al., 2018). The mass spectrometer scanned either in full MS mode, for the 3 min method, or performed acquisition independent fragmentation, for the $9 \mathrm{~min}$ method, at 70,000 resolution in the 70-900 m/z range, 4 $\mathrm{kV}$ spray voltage, 15 sheath gas and 5 auxiliary gas, operated in negative and then positive ion mode as separate runs (Jones et al., 2018). Metabolite assignment was performed against an inhouse standard library. Metabolite levels were then normalized to protein quantification (Jones et al., 2018). Peak areas and isotopologue deconvolution were determined as described (Jones et al., 2018).

\subsection{In Vivo Studies}

Non-obese diabetic/ severe combined immunodeficiency y (NOD/SCID gamma or NSG; Jackson Laboratory) mice were used for xenotransplant and engraftment studies and conducted as previously described (Lee et al., 2015). A oil-in-water emulsion containing AYNE was used for treating mice (Ahmed et al., 2020). In brief, $50 \mu \mathrm{L}$ of NeoBeeM5 (Stepan) and $50 \mu \mathrm{L}$ of Tween 80 (P1754; Sigma) were heated at $75^{\circ} \mathrm{C}$ for two hours with 1-20 mg AYNE with gentle agitation on a lab gyrator. AYNE is omitted in the vehicle emulsion. The $100 \mu \mathrm{L}$ oil phase was dispersed into $900 \mu \mathrm{L}$ of PBS and the total of $1 \mathrm{~mL}$ emulsion was vortexed vigorously for 30 seconds. All animal studies were carried out according to the regulations of the Canadian Council on Animal Care and with the approval of the University of Guelph, Animal Care Committee under the animal utilization protocol \#3635. 


\subsubsection{In Vivo Tumor Formation}

$1 * 10^{\wedge} 6$ cells AML2 cells solvated in $100 \mu \mathrm{L}$ PBS were subcutaneously injected into the mouse's right flank. Once tumors were palpable, mice were divided into groups to receive a vehicle emulsion or an emulsion (Ahmed et al., 2020) containing either $150 \mathrm{mg} / \mathrm{kg}$ of the $(2 R, 4 R)-$ or $(2 S, 4 S)$-AYNE stereoisomer every other day for two weeks via an intra-peritoneal injection. Tumor volumes were measured as previously described (Lee et al., 2015) and tumor burden was calculated relative to each mouse's weight.

\subsubsection{In Vivo Engraftment}

$2.5^{*} 10^{\wedge} 6$ cells human AML cells solvated in $100 \mu \mathrm{L}$ PBS were injected via tail vein and engrafted into the femurs over the course of one or four weeks. Mice received either a vehicle emulsion (Ahmed et al., 2020) or an emulsion (Ahmed et al., 2020) containing $200 \mathrm{mg} / \mathrm{kg} / \mathrm{week}$ of $(2 R, 4 R)$-AYNE for four weeks. Human leukemia cells in the femurs were recovered, incubated with anti-CD33 (Cat\#303404; Biolegend) and anti-CD45 (Cat\#103107; BioLegend) antibodies, and then quantified via flow cytometry on a Guava EasyCyte 8HT.

\subsection{Cellular Thermal Shift Assay}

To quantify thermal stabilization of VLCAD by AYNE (Ishii et al., 2017), $2.5^{*} 10^{6}$ cells of human AML cells were injected via tail vein into a NSG mouse and allowed eight weeks to engraft and propagate in the femurs. Mice were treated with a bolus dose of a vehicle emulsion or an emulsion containing AYNE (150 or $200 \mathrm{mg} / \mathrm{kg}$ ) 24 hours prior to a planned sacrifice. Human AML recovered from mice treated with a bolus dose of AYNE were resuspended in pre-chilled RIPA buffer $+1 \mathrm{X}$ protease inhibitor, flash frozen in liquid nitrogen three times, and centrifuged at 12,000 rpm for 20 minutes. The supernatant was carefully removed and transferred to a polymerase chain reaction (PCR) tube (TBC0802; Bio-Rad). In a thermocycler (MyCycler; BioRad), the sample was 
subjected to a heat shock at $60^{\circ} \mathrm{C}$ for 3 minutes followed by a drop to $4^{\circ} \mathrm{C}$ for 3 minutes. The supernatant was centrifuged a second time at $21,000 * \mathrm{~g}$ for $1 \mathrm{hr}$. With extreme care to not disturb the non-visible pellet, the supernatant was recovered and diluted with SDS-PAGE sample buffer for immediate SDS-page analysis.

\subsection{Drug Combination Analysis By Calcusyn}

The combination index $(\mathrm{CI})$ evaluated the interaction between the constituents of avo-B and either Ara-C or DOX. TEX and AML2 cells were treated with increasing concentrations of AYNE, AENE, ara-C, DOX as a single agent or in combinations. Cell death after 72 hours was assessed by the MTS assay. CI values, generated by the Calcusyn median effect model, determined if combinations were either synergistic, antagonistic or additive. CI values of $<1$ indicate synergism, $\mathrm{CI}=1$ indicate additivity and CI $>1$ indicate antagonism (Chou, 2010).

\subsection{Statistical Analysis In GraphPad Prism 7.0}

Unless otherwise stated, results are presented as mean \pm SD. Data was analyzed with GraphPad Prism 7.0 (GraphPad Software) using an ANOVA with Tukey's post hoc analysis for between group comparisons or standard Student's $t$-tests where appropriate and Mann-Whitney t-

tests for animal experiments. ${ }^{*} \mathrm{p} \leq 0.05 ; * * \mathrm{p} \leq 0.002 ; * * \mathrm{p} \leq 0.001$. $\mathrm{IC}_{50}$ values are presented as mean \pm standard deviation are calculated using the $\log$ (inhibitor) vs. normalized response -Variable slope function in GraphPad Prism 7.0. 


\subsection{Chapter 4 Avocadyne (AYNE) Is The Anti-AML Component Of Avo-B,}

\section{An Avocado-Derived Mixture Of Fatty Alcohols, That Selectively Eliminates}

\section{AML}

Matthew Tcheng1, Ismael Samudio ${ }^{2}$, Eric A. Lee ${ }^{1}$, Mark Minden ${ }^{3}$, Paul A. Spagnuolo ${ }^{1 *}$

${ }^{1}$ School of Pharmacy, University of Waterloo, 10A Victoria Street, Kitchener, Ontario, Canada, $\mathrm{N} 2 \mathrm{G} 1 \mathrm{C} 5$

${ }^{2}$ Centre for Drug Research and Development, Vancouver BC

${ }^{3}$ Princess Margaret Cancer Center, Ontario Cancer Institute, 610 University Ave, Toronto ON, M5G 2M9, Canada;

* Correspondence email: paul.spagnuolo@uoguelph.ca

Modified version of this manuscript is:

1. In press at the Journal of Leukemia \& Lymphoma under the title "The mitochondria target drug avocatin-B synergizes with induction chemotherapeutics to induce leukemia cell death"

2. Under review at the Journal of Food Biochemistry under the title "Avocado-derived Avocadyne is the Most Potent Inhibitor of Fatty Acid Oxidation" 


\subsection{Introduction}

Mitochondrial oxidative metabolism in AML is rewired to increase proliferation, clonogenic potential, engraftment capacity as well as resistance to apoptosis activation upon chemotherapeutic stress (Samudio et al., 2010)(Kuntz et al., 2017)(German et al., 2016)(Stevens et al., 2020). Several in vitro (Muus et al., 1991) and in vivo (Farge et al., 2017)(Stevens et al., 2020) studies demonstrated that chemotherapy triggers an energy crisis in surviving AML cells, resulting in increased FAO activity coupled to OXPHOS to avert ATP depletion.

Ara-C, a frontline anti-AML chemotherapeutic, kills the rapidly dividing AML population through the inhibition of DNA polymerase. At $0.05 \mu \mathrm{M}$, the same concentration capable of inhibiting DNA synthesis, ara-C induced upregulation of cytochrome C oxidase (CIV), resulting in increased mitochondrial respiration in the AML cell line Molt-4 (Muus et al., 1991). More recently, the relapse initiating population that survived cytarabine and the venetoclax/azacytidine regimen increased OXPHOS and FAO gene expression to maintain ATP levels, compared to de novo AML cells. FAO inhibition re-sensitized resistant AML cells to these chemotherapies, resulting in AML death (Farge et al., 2017)(Stevens et al., 2020). The FAO inhibitors used in these studies are not approved for anti-AML use, underscoring the need to identify well-tolerated novel FAO inhibitors that induce death in AML populations synergistically with current chemotherapeutics. 
A

Non-transformed

\subsection{Results}
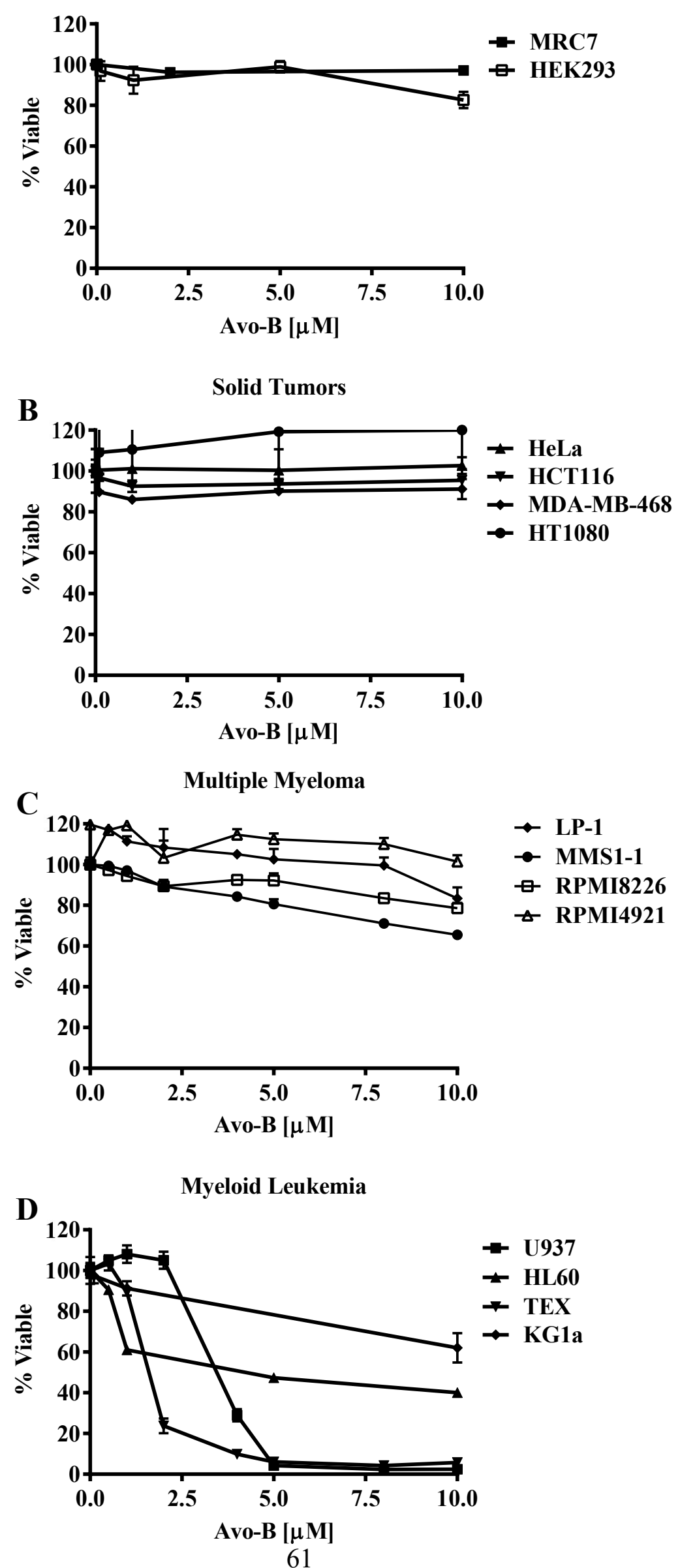
Figure 4.2.1 Avo-B Selectively Kills AML Cell Lines

Cell viability of human immortalized (A) lung MRC7, kidney HEK293 (B) cervical cancer HeLa, colon cancer HCT116, breast cancer MDA-MB-486, connective tissue cancer HT1080 (C) multiple myeloma LP-1, MMS1-1, RPMI8226, RPMI4921 (D) myeloid leukemia U937, HL60, TEX, KG1a cell lines treated with avo-B for 72 hours assessed by the MTS assay $(\mathrm{N}=3)$. IC $\mathrm{C}_{50}$ values for AML lines were tabulated in Table 4.2.1. If cell viability did not decrease below 50\% in the concentration range tested, no $\mathrm{IC}_{50}$ value is calculated. $\mathrm{IC}_{50}$ values are presented as mean \pm standard deviation and were calculated using the $\log$ (inhibitor) vs. normalized response -- Variable slope function in GraphPad Prism 7.0. Experiments conducted by E. A. Lee.

Table 4.2.1 IC50 Values Of Avo-B On Myeloid Leukemia Cell Lines.

\begin{tabular}{|c|c|c|c|c|}
\hline Cell Line & U937 & HL60 & TEX & KG1a \\
\hline $\mathrm{IC}_{50}(\mu \mathrm{M})$ & $3.68 \pm 0.26$ & $4.39 \pm 0.32$ & $1.57 \pm 0.19$ & Did not converge \\
\hline
\end{tabular}




\subsubsection{Avo-B Selectively Kills AML Cell Lines}

Following a 72-hour avo-B treatment, the viability of non-transformed, solid tumor, multiple myeloma, and myeloid leukemia cell lines was assessed. Across the concentration range tested, avo-B failed to induce at least 50\% cell death in all non-transformed (Figure 4.2.1 A), solid tumor (Figure 4.2.1 B), and multiple myeloma (Figure 4.2.1 C) cell lines. Avo-B demonstrated cytotoxicity exclusively against AML cell lines (Figure 4.2.1 D), producing $\mathrm{IC}_{50}$ values below 5 $\mu \mathrm{M}$ for three out of the four cell lines tested (Table 4.2.1). 
A

Primary AML

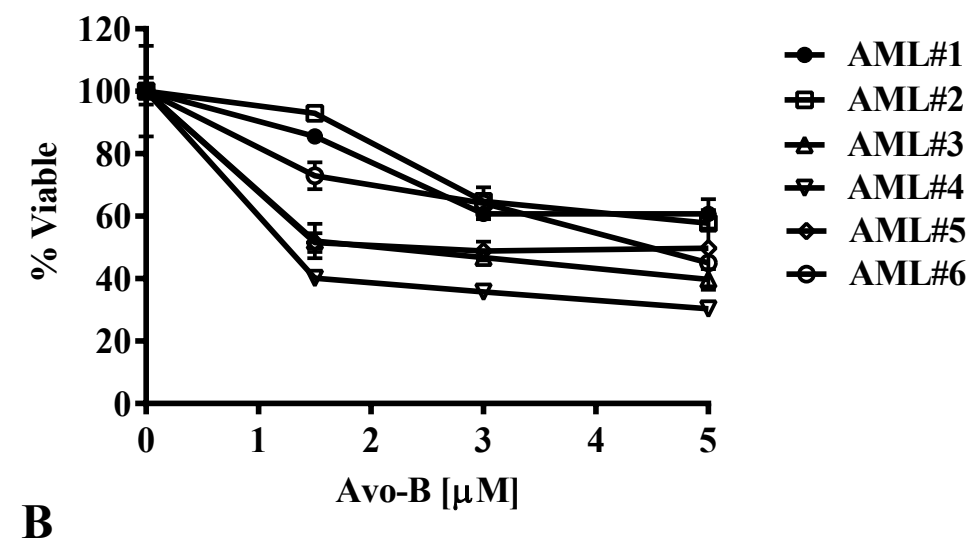

B

Normal Mononuclear Cells (MNC)

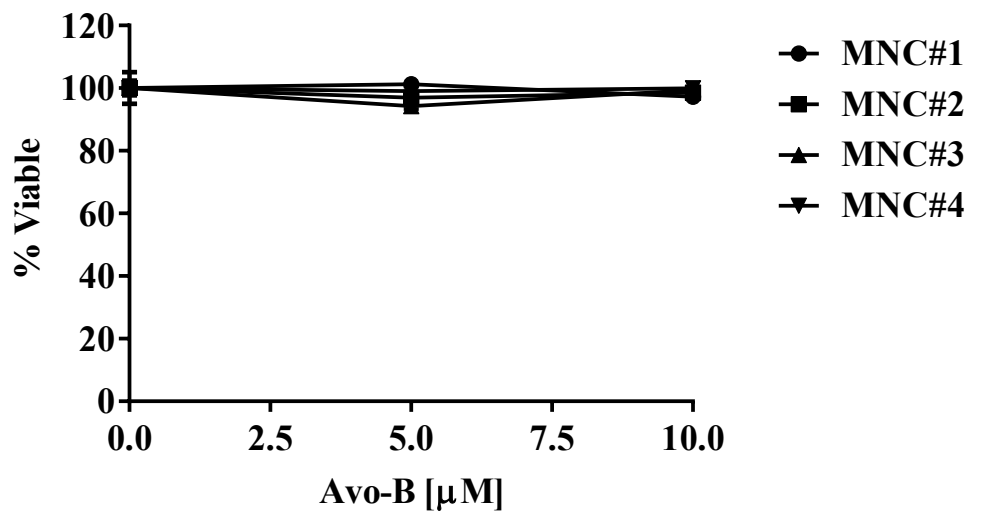

Figure 4.2.2 Avo-B induces death of primary AML cells, while sparing normal MNC cells.

Cell viability of human (A) patient derived primary AML $(\mathrm{N}=6)$ and $(\mathbf{B})$ donor derived primary MNC $(\mathrm{N}=4)$ treated with avo-B for 72 hours assessed by flow cytometry using the 7AAD stain. See Table 4.2.5 for cytogenetics of patient IDs. Experiments conducted by E. A. Lee. 


\subsubsection{Avo-B Induces Death Of Primary AML Cells, While Sparing Normal MNC Cells}

Immortalized cell lines are critical to demonstrating pre-clinical efficacy of potential therapeutics due to their rapid and consistent growth rates (Kaur \& Dufour, 2012). While immortalized cell lines are homogenous, primary patient-derived AML cells are more reflective of the heterogeneity of AML in the clinical human disease (Kaur \& Dufour, 2012) and are therefore critical tools to demonstrate the pre-clinical efficacy of avo-B. Normal MNC cells from healthy (non-AML patient) donors are also critical controls to ensure non-transformed hematopoietic cells can tolerate potential anti-AML therapeutics. At the same concentration and timepoint, avo-B demonstrated cytotoxicity against primary AML cells (Figure 4.2.2 A) while donor-derived hematopoietic cells were unharmed (Figure 4.2.2 B), demonstrating a therapeutic window of targeting AML cells with avo-B. 

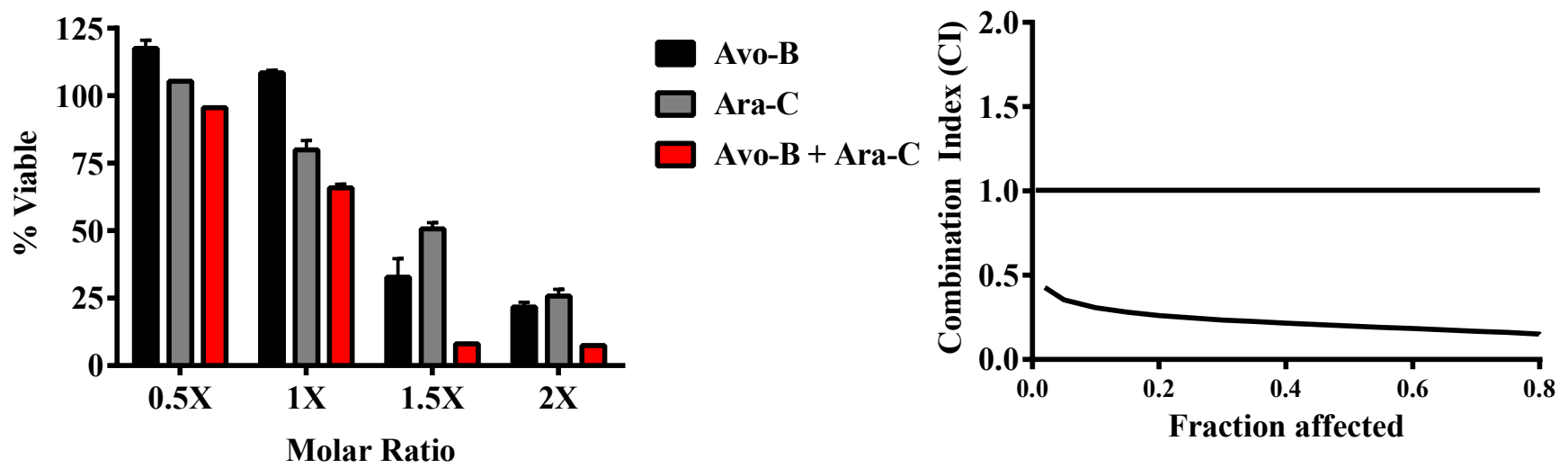

B

Combination of Avo-B and DOX

D

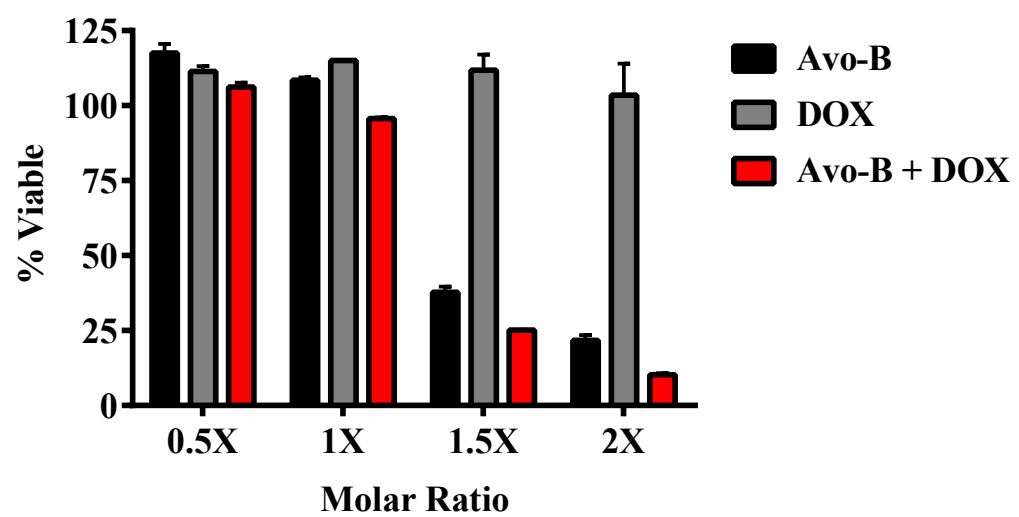

Combination of Avo-B and DOX: Calculsyn

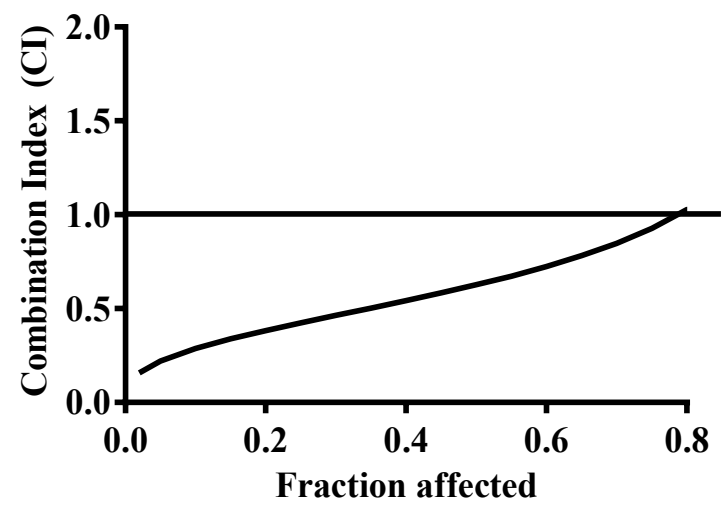

Figure 4.2.3 Avo-B synergizes with induction chemotherapeutics Ara-C and DOX to kill TEX.

Viability of TEX cells treated with different $\mathrm{IC}_{50}$ ratios of Avo-B and either (A) Ara-C or (B) DOX for 72 hours with the MTS (N=3). Assessment of drug interaction between Avo-B and either (C) Ara-C or (D) DOX in Calcusyn using the Chou-Talalay method (Chou, 2010) where a combination index (CI) (Y-axis) value of $<1,=1,>1$ denotes both drugs are synergistic, exhibit an additive effect, or are antagonistic at inducing TEX cytotoxicity. $\mathrm{CI}=1$ is marked with a horizontal line in $(\mathbf{C}, \mathbf{D})$. Fraction affected and CI values used to plot $(\mathbf{C}, \mathbf{D})$ are tabulated in Table 4.2.2. Experiments conducted by E. A. Lee.

Table 4.2.2 Combination index values with combinations of avo-B and induction chemotherapeutics in TEX cells.

\begin{tabular}{|c|c|c|}
\hline Fraction affected & $\begin{array}{c}\text { Combination Index (C.I.) } \\
\text { Avo-B + DOX Combo }\end{array}$ & $\begin{array}{c}\text { Combination Index (C.I.) } \\
\text { Avo-B + Ara-C Combo }\end{array}$ \\
\hline 0.3 & 0.4 & 0.2 \\
\hline 0.5 & 0.6 & 0.19 \\
\hline 0.7 & 0.8 & 0.15 \\
\hline
\end{tabular}




\subsubsection{Avo-B Synergizes With Induction Chemotherapeutics To Induce AML Cytotoxicity}

Demonstrating a synergistic or an additive effect between avo-B, a pre-clinical therapeutic, as well as ara-C and DOX, the backbones of the $7+3$ regimen, further establishes the pre-clinical efficacy of targeting AML mitochondrial metabolism. Cell death (Figure 4.2.3 A, B) was assessed by the drug interaction software Calcusyn. Calcusyn was used to evaluate whether the combinations of avo-B with either chemotherapeutic was synergistic, additive, or antagonistic (Chou, 2010). The drug interaction algorithm assesses the combined effect on AML cytotoxicity of both agents under fixed molar ratios and generates a combination index $(\mathrm{CI})$ value as a function of AML cell death. $\mathrm{CI}$ is calculated based on the following formula: $\mathrm{CI}=\mathrm{C}_{\mathrm{a}, \mathrm{x}} / \mathrm{IC}_{\mathrm{x}, \mathrm{a}}+\mathrm{C}_{\mathrm{b}, \mathrm{x}} / \mathrm{IC}_{\mathrm{x}, \mathrm{b}}$ where $\mathrm{C}_{\mathrm{a}, \mathrm{x}}$ and $\mathrm{C}_{\mathrm{b}, \mathrm{x}}$ are the drug a and $\mathrm{b}$ concentrations used in combination to impart a drug effect and $\mathrm{IC}_{\mathrm{x}, \mathrm{a}}$ and $\mathrm{IC}_{\mathrm{x}, \mathrm{b}}$ are the concentrations of the drug when used individually (Chou, 2010). CI values of $<1$ indicate synergism, $\mathrm{CI}=1$ indicate additivity and $\mathrm{CI}>1$ indicate antagonism (Chou, 2010). The CI values for combinations of avo-B and either DOX or ara-C are visualized in Figure 4.2.3 C, D and tabulated in Table 4.2.2. CI values for the avo-B/ara-C and the avo-B/DOX combinations were all below 1.0 (Table 4.2.2), demonstrating strong synergy in eliminating TEX, further demonstrating efficacy in targeting mitochondrial metabolism in AML. 
TEX

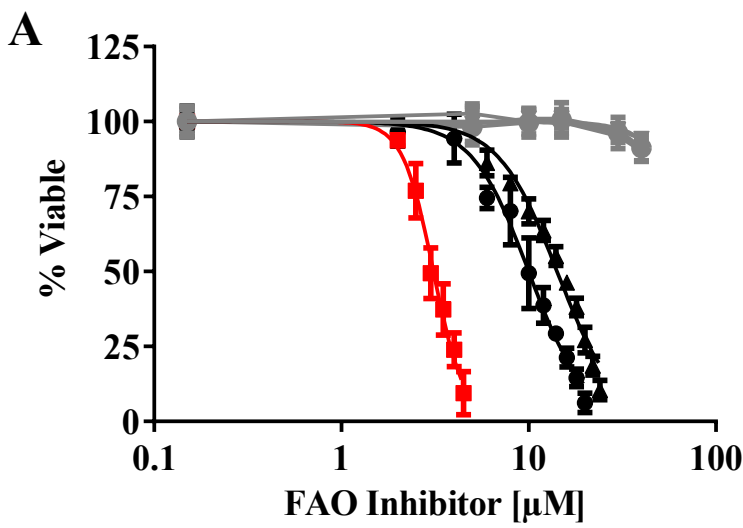

C

TEX

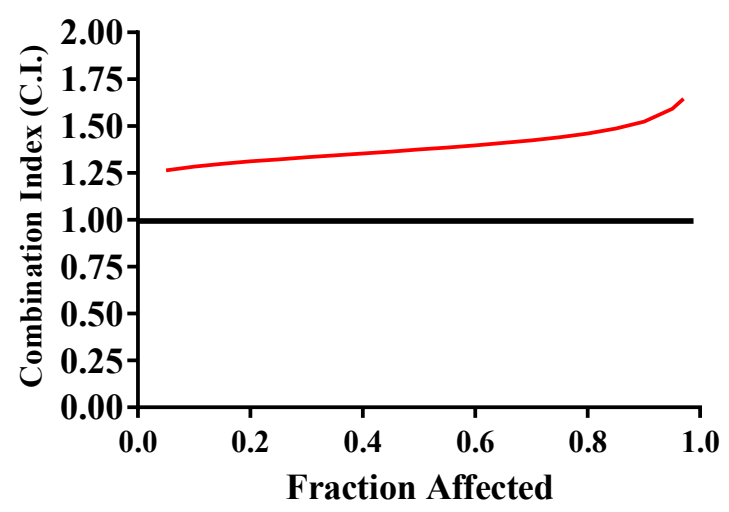

$\mathbf{E}$

AML Clonogenic Potential

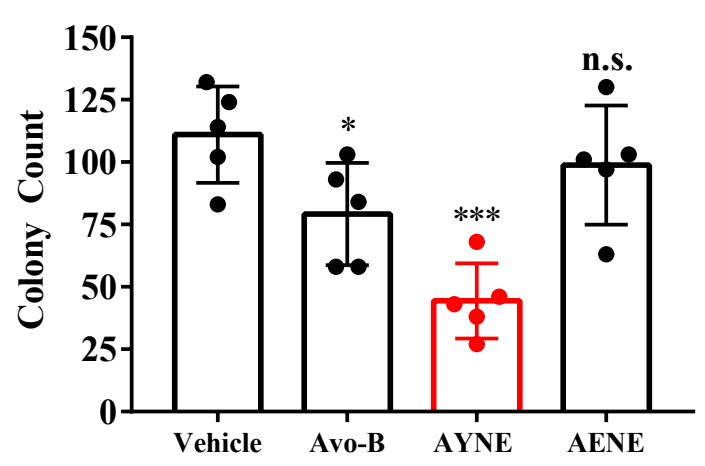

AML2

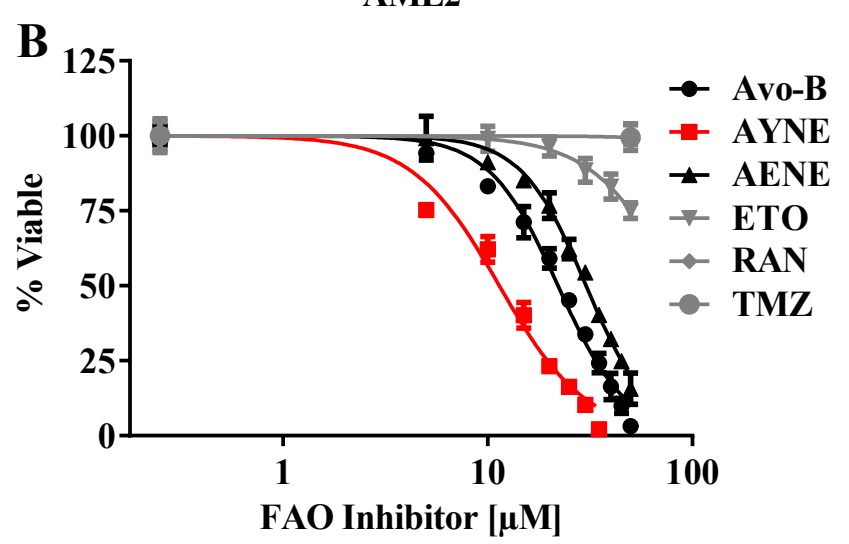

D

AML2

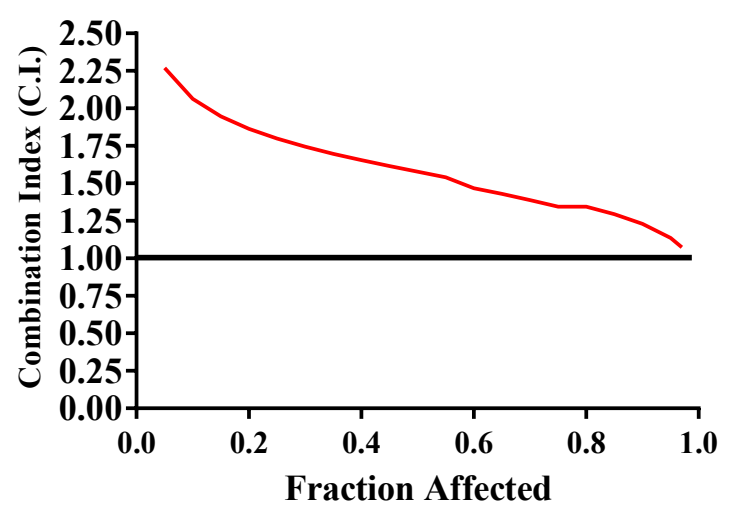

F Normal MNC Clonogenic Potential

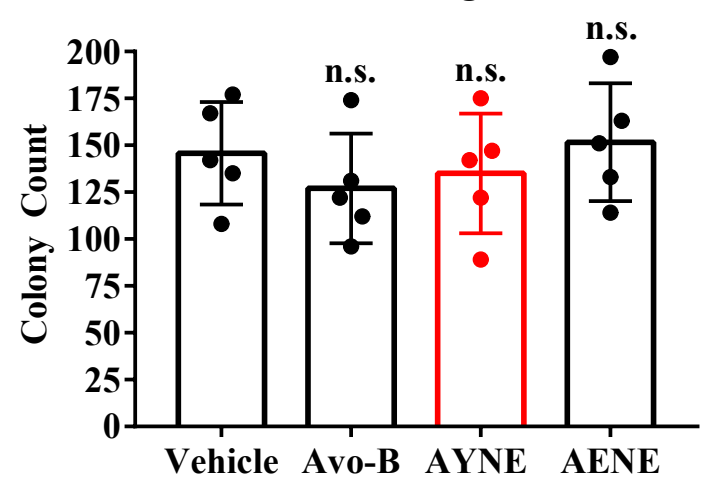

Figure 4.2.4 The constituents of avo-B induce AML death in an antagonistic manner Cell viability of (A) TEX and (B) AML2 treated for 72 hours with avo-B, AYNE, AENE, ETO, RAN or TMZ analyzed by flow cytometry with a 7-AAD stain $(\mathrm{N}=3)$. AYNE is highlighted with a red line; avo-B and AENE are black lines; other FAO inhibitors are grey lines. $\mathrm{IC}_{50}$ values are tabulated in Table 4.2.3. Assessment of drug interaction between the constituents of avo-B on (C) TEX and (D) AML2 in Calcusyn using the Chou-Talalay method (Chou, 2010) where a combination index (CI; Y-axis) value of $<1,=1,>1$ denotes both drugs are synergistic, exhibit 
an additive effect, or are antagonistic at inducing $\mathrm{AML}$ cytotoxicity. $\mathrm{CI}=1$ is marked with a horizontal line in $(\mathbf{C}, \mathbf{D})$. CI's are tabulated in Table 4.2.4. Clonogenic potential of (E) primary patient-derived AML (AML\#7-11) and (F) normal donor-derived MNC samples (N=5) after a 10 $\mu \mathrm{M}$ treatment of either avo-B, AYNE, or AENE for 14 days. AYNE is highlighted with a red border. For $(\mathbf{A}, \mathbf{B})$, if cell viability did not decrease below $50 \%$ in the concentration range tested, no $\mathrm{IC}_{50}$ value is calculated. $\mathrm{IC}_{50}$ values are presented as mean \pm standard deviation and were calculated using the $\log$ (inhibitor) vs. normalized response -- Variable slope function in GraphPad Prism 7.0. For $(\mathbf{E}, \mathbf{F})$, data presented as mean \pm S.D., ${ }^{*} p<0.05, * * p<0.01, * * * p<0.001$ using an unpaired, one-tailed ANOVA calculated in GraphPad Prism 7.0. See Table 4.2.5 for cytogenetics of patient IDs.

Table 4.2.3 Summary of $I_{50}$ values of the avo-B mixture and its constituents AYNE and AENE on TEX and AML2 presented in figure 3.2.4 A, B.

\begin{tabular}{|c|c|c|c|}
\hline & Avo-B $(\mu \mathrm{M})$ & AYNE $(\mu \mathrm{M})$ & AENE $(\mu \mathrm{M})$ \\
\hline TEX IC 50 (Mean \pm STDEV) & $9.99 \pm 1.69$ & $3.10 \pm 0.14$ & $14.19 \pm 1.06$ \\
\hline AML2 IC 50 (Mean \pm STDEV) & $22.04 \pm 2.05$ & $11.53 \pm 3.32$ & $30.25 \pm 2.24$ \\
\hline
\end{tabular}

Table 4.2.4 Summary of combination index values for the avo-B mixture on TEX and AML2 cell lines presented in figure 3.2.4 C, D.

\begin{tabular}{|c|c|c|}
\hline Fraction affected & $\begin{array}{c}\text { CI values for avo-B on } \\
\text { TEX }\end{array}$ & $\begin{array}{c}\text { CI values for avo-B on } \\
\text { AML2 }\end{array}$ \\
\hline 0.2 & 1.31 & 1.86 \\
\hline 0.4 & 1.35 & 1.65 \\
\hline 0.6 & 1.39 & 1.47 \\
\hline 0.8 & 1.46 & 1.35 \\
\hline
\end{tabular}




\subsubsection{The Constituents Of Avo-B Induce AML Death In An Antagonistic Manner}

In a panel of containing the constituents of avo-B AYNE and AENE, the avo-B mixture, and 3 previously established FAO inhibitors, AYNE, the constituent of avo-B with a terminal triple bond, was the most cytotoxic compound, exhibiting an $\mathrm{IC}_{50}$ of $3.10 \pm 0.12 \mu \mathrm{M}$ and $11.39 \pm 0.90$ $\mu \mathrm{M}$ on TEX and AML2, respectively (Figure 4.2.4 A, B, Table 4.2.3). FAO inhibitors ETO, RAN, and $\mathrm{TMZ}$ did not reduce cell viability below 50\% across the concentration range tested, in agreement with previous studies (Samudio et al., 2010) (Shafat et al., 2017). Following a 72-hour treatment with either AYNE, AENE, or the avo-B mixture, Calculsyn was used to assess TEX and AML2 death. When AYNE and AENE, the constituents of avo-B, were tested as single agents and in combination, the combinations produced CI values $>1$, demonstrating antagonism while inducing AML cytotoxicity on TEX (Figure 4.2.4 C, Table 4.2.4) and AML2 (Figure 4.2.4 D, Table 4.2.4). Taken together, AYNE is more cytotoxicity to AML as a single agent, compared with the avo-B mixture or AENE.

The colony formation assay involves culturing primary human leukemia and hematopoietic stem and progenitor cells on semisolid media to assess long term (in this study, two weeks) proliferation and differentiation potential (Sarma et al., 2010). Current induction chemotherapeutics are toxic to both leukemia and normal hematopoietic populations, resulting in hematopoietic pancytopenia, a common co-morbidity of AML treatment, underscoring the need to assess the effect of novel chemotherapeutics on leukemia and hematopoietic clonogenic growth (Shipley \& Butera, 2009). AYNE and avo-B significantly decreased AML colony counts (Figure 4.2.4 E: $F(3,15)=11.16, p<0.001)$, while normal clonogenic growth was spared (Figure 4.2.4 F: $\mathrm{F}(3,15)=1.98, \mathrm{p}=0.17)$. Taken together, avo-B induced death in AML cells in an antagonistic manner. AYNE was the most cytotoxic component in avo-B and, as a single agent, selectively 
killed AML while sparing normal blood cells, a pre-requisite of any novel anti-AML chemotherapeutic. 


\section{$\left[16-{ }^{14} \mathrm{C}\right]-$ Palmitic acid coupled to BSA}
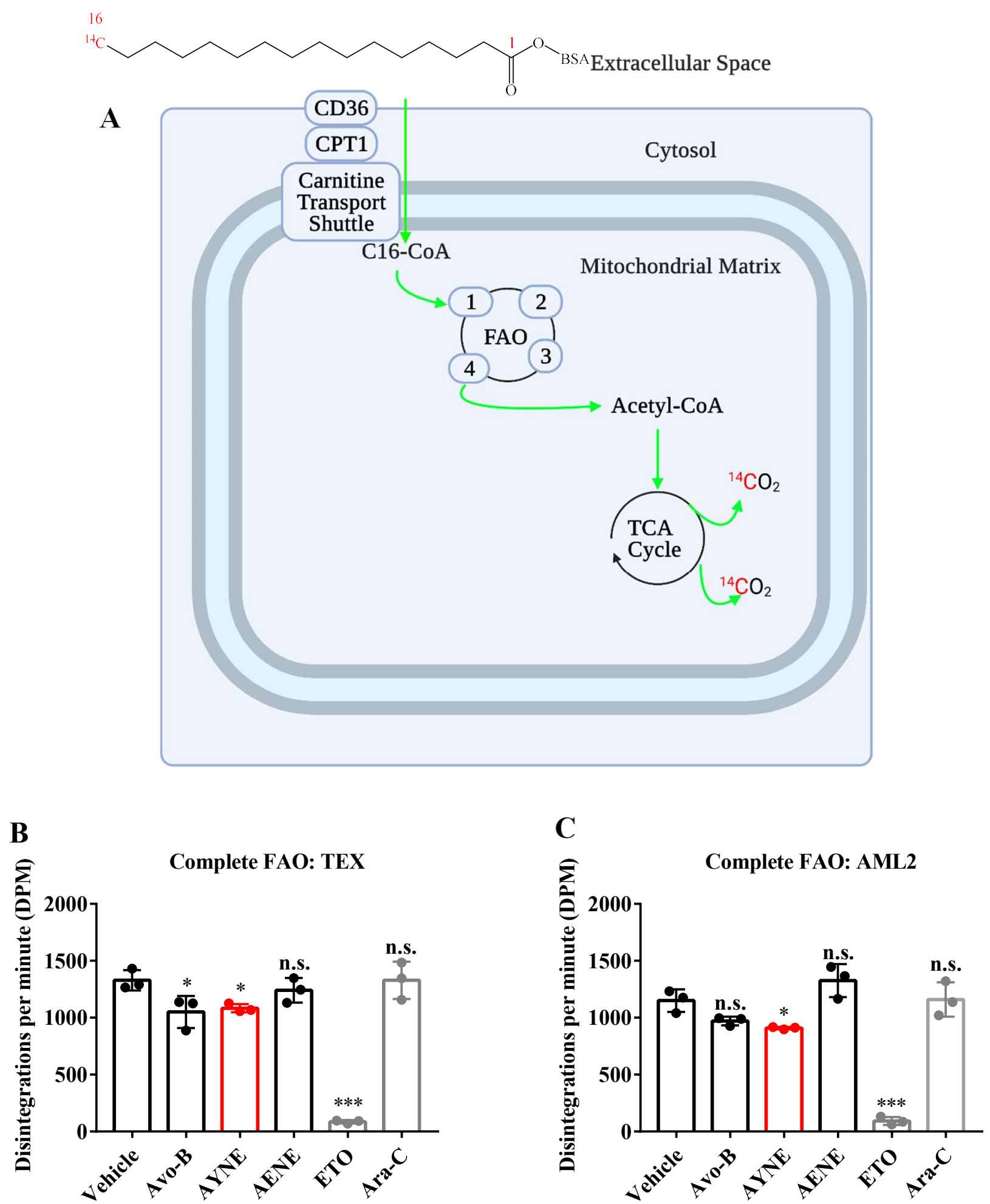

Figure 4.2.5 AYNE inhibits mitochondrial long chain FAO in AML.

(A) Schematic showing the assessment of long chain FAO in leukemia cells via liquid scintillation counting (Huynh et al., 2014). Briefly, $\left[16-{ }^{14} \mathrm{C}\right]$-palmitate coupled to bovine serum albumin (BSA) (henceforth referred to as radiopalmitate; the path of oxidation is highlighted in green) is supplied to leukemia cells. Radiopalmitate is completely oxidized by the mitochondrial FAO pathway and, 
following TCA metabolism, the released ${ }^{14} \mathrm{CO}_{2}$ is quantified via liquid scintillation counting, as a direct measure of FAO. Abbreviations: BSA, bovine serum albumin; CD36, cluster of differentiation 36; CPT1, carnitine palmitoyltransferase 1; CoA, coenzyme A; C16-CoA, palmitoyl-CoA; FAO, fatty acid oxidation; TCA, tricarboxylic acid. Created in Biorender. Rates of long chain mitochondrial FAO in (B) TEX and (C) AML2 following a three hour treatment with either a vehicle, $10 \mu \mathrm{M}$ avo-B, AYNE, AENE, $100 \mu \mathrm{M}$ ETO (positive control for FAO inhibition), $1 \mu \mathrm{M}$ ara-C (negative control for FAO inhibition). AYNE is highlighted with a red border. For $(\mathbf{B}, \mathbf{C})$, data presented as mean \pm S.D., ${ }^{*} \mathrm{p}<0.05, * * \mathrm{p}<0.01, * * * \mathrm{p}<0.001$ using an unpaired, one-tailed ANOVA calculated in GraphPad Prism 7.0. For $(\mathbf{B}, \mathbf{C})$, experiments were performed by K. Rea and Prof. T. A. Akhtar (University of Guelph). 


\subsubsection{AYNE Inhibits Mitochondrial Long Chain FAO To Induce AML Death}

Previously, avo-B inhibited fat-supported mitochondrial respiration to kill AML cells (Lee et al., 2015). Liquid scintillation counting assesses the rate of mitochondrial FAO that is proportional to the amount of released ${ }^{14} \mathrm{CO}_{2}$; the ${ }^{14} \mathrm{C}$ atom originates from exogenous radiopalmitate (Figure 4.2.5 A)(Huynh et al., 2014). AYNE alone directly inhibited FAO in both TEX (Figure 4.2.5 B: $F(5,17)=59.17, p=0.01)$ and AML2 (Figure 4.2.5 C: $F(5,17)=61.53, p=$

0.03). Taken together, AYNE hinders mitochondrial respiration (Lee et al., 2015) by inhibiting FAO. However, additional studies are required to identify a molecular target along the FAO pathway. 
Table 4.2.5 Cytogenetics for patient IDs for Chapter 4

\begin{tabular}{|c|c|}
\hline Patient ID & Cytogenetics \\
\hline AML\#1 & $\begin{array}{c}43 \sim 44, X X, \operatorname{dic}(3 ; 12)(\operatorname{p} 13 ; p 11.2),-5,- \\
7,+\operatorname{del}(11)(\mathrm{q} 13), ? \operatorname{dup}(18)(\mathrm{q} 21 \mathrm{q} 22), \operatorname{del}(20)(\mathrm{q} 13.1),-22,+\operatorname{mar}[\mathrm{cp} 10]\end{array}$ \\
\hline AML\#2 & $44 \sim 48, \mathrm{XX}, \operatorname{del}(5)(\mathrm{q} 13 \mathrm{q} 33), \mathrm{i}(9)(\mathrm{p} 10),-18, \operatorname{add}(20)(\mathrm{q} 13.3),+\operatorname{mar}[\mathrm{cp} 17] / 46, \mathrm{XX}[3]$ \\
\hline AML\#3 & 46,XX,t(2;8)(p13;q22)[20].ish t(2;8)(MYC+;MYC-) \\
\hline AML\#4 & NPM, Flt3-TKD \\
\hline AML\#5 & Unsuccessful \\
\hline AML\#6 & Del 5q \\
\hline AML\#7 & $46, \mathrm{XX}[20]$ \\
\hline AML\#8 & 46,XY,t(1;12)(q21;p12 13),?add(4)(p16)[3]/47,idem,+9[7] \\
\hline AML\#9 & $46, X Y[20]$ \\
\hline AML\#10 & $46, \mathrm{XX}[20]$ \\
\hline AML\#11 & Not Tested \\
\hline
\end{tabular}




\subsection{Discussion}

Rational drug combination design dictates that the agent being added to the treatment regimen must synergize with the mechanism of action of the current regimen (Ramaswamy, 2007). For example, AML cells resistant to cytarabine (Farge et al., 2017) and venetoclax/azacytdine (Stevens et al., 2020) previously upregulated FAO and oxidative metabolism to increase ATP and TCA metabolite production, resulting in survival and continued proliferation. This suggests an FAO inhibitor is a logical third agent to these regimens. Unfortunately, inhibition of FAO by shRNA as well as the FAO inhibitors ETO and SSO, are not clinically approved for human use (Farge et al., 2017)(Stevens et al., 2020). Clinically approved FAO inhibitors, such as RAN and TMZ, required high millimolar concentrations for anti-AML activity (Samudio et al., 2010). The results presented in this chapter investigated the hypotheses set out in chapter 2 to determine that the avo-B mixture was antagonistic and that avocadyne was the most active anti-AML agent. These results demonstrated specificity of avo-B towards FAO-dependent cancers, such as AML, and confirmed that avo-B synergized with current induction therapies to induce AML death and was welltolerated by normal hematopoietic cells. The results of the liquid scintillation counting experiments confirm that avocadyne killed AML cells through the inhibition of FAO; further pharmacodynamic studies are required to elucidate a mechanism of action and confirm a molecular target.

Antagonism between constituents of therapeutic regimens must be avoided to maximize efficacy against cancer cells (Chou, 2010). For example, a combination of methotrexate and cytarabine demonstrated antagonism due to opposing mechanisms of action on the leukemia cell cycle (Akutsu et al., 2002). As single agents, methotrexate accumulated cells in the $\mathrm{G}_{1}$ to early $\mathrm{S}$ phase while cytarabine accumulated cells in the late $S$ and early $G_{2}$ phases; these opposing effects 
on the cell cycle reduced cytotoxicity of the combination, compared to the single agents alone (Akutsu et al., 2002).

Natural products such as epigallocatechin gallate (Modernelli et al., 2015) and quercetin (Liu et al., 2008) antagonized the activity of bortezomib, a frontline therapeutic used to treat Bcell lymphoma, chronic lymphocytic leukemia, and multiple myeloma, by forming a complex with its boron moiety, required for its proteasome inhibitory activity. Antagonism between two compounds from the same food product, as shown in this chapter between avocado-derived AYNE and AENE, has not been previously reported. Taken together, these results confirm that the avo-B mixture synergized with current induction chemotherapeutics to selectively eliminate AML. While AYNE was the most potent FAO inhibitor and most cytotoxic avo-B constituent towards AML, additional experiments are required to identify an enzyme target and demonstrate anti-AML efficacy in vitro and in vivo. 


\subsection{Chapter 5 The Very Long Chain Acyl-CoA Dehydrogenase (VLCAD)}

\section{Is The Molecular Target Of Avocadyne (AYNE)}

Matthew Tcheng ${ }^{1}$, Alessia Roma ${ }^{1}$, Nawaz Ahmed ${ }^{1}$, Richard W. Smith ${ }^{2}$, Preethi Jayanth ${ }^{1}$, Mark D. Minden ${ }^{3}$, Aaron D. Schimmer ${ }^{3}$, David Hess ${ }^{4}$, Kristin Hope ${ }^{5}$, Kevin Rea ${ }^{6}$,Tariq A. Akhtar ${ }^{6}$, Eric Bohrnsen $^{7}$, Angelo D’Alessandro ${ }^{7}$, Al-Walid Mohsen ${ }^{8,9}$, Jerry Vockley, ${ }^{8,9}$, and Paul A. Spagnuolo $^{1 *}$

${ }^{1}$ Department of Food Science, University of Guelph, 50 Stone Rd. Guelph Ontario, Canada. N1G2W1

${ }^{2}$ University of Waterloo Mass Spectrometry Facility, Department of Chemistry, 200 University Avenue West, Waterloo, ON, N2L 3G1, Canada

${ }^{3}$ Princess Margaret Cancer Center, Ontario Cancer Institute, 610 University Ave, Toronto ON, M5G 2M9, Canada;

${ }^{4}$ University of Western Ontario, Robarts Research Institute, 100 Perth Drive, London, ON N6A $5 \mathrm{~K} 8$

${ }^{5}$ Stem Cell and Cancer Research Institute (SCC-RI). McMaster University, 1280 Main Street West, Hamilton, ON L8S 4K1.

${ }^{6}$ Department of Molecular and Cellular Biology, University of Guelph, Guelph Ontario, Canada

${ }^{7}$ Department of Biochemistry and Molecular Genetics, University of Colorado Anschutz Medical Campus, Aurora, CO, USA 80045

${ }^{8}$ Department of Pediatrics and Center for Rare Disease Therapy, UPMC, Children's Hospital of Pittsburgh, University of Pittsburgh, Pittsburgh, PA 15224

${ }^{9}$ Department of Human Genetics, School of Public Health, University of Pittsburgh, Pittsburgh, PA 15260

* Correspondence email: paul.spagnuolo@uoguelph.ca

Modified version of this manuscript is available online ahead of press at the Blood journal under the title: "Very long chain fatty acid metabolism is required in acute myeloid leukemia" 


\subsection{Introduction}

Compared to the normal hematopoietic population, the dysregulation of cell proliferation, differentiation and survival is driven by an overreliance on higher rates of FAO in the AML population that can be pharmacologically targeted (Samudio et al., 2010)(German et al., 2016)(Shafat et al., 2017)(Zhang et al., 2020)(Wu et al., 2015). There are two primary reasons to consider targeting abnormally upregulated FAO to induce AML death.

Driven by the dysregulation of upstream regulators, such as PHD3 under-expression (German et al., 2016) and PML overexpression (Ito et al., 2008), high rates of FAO in AML supply acetyl-CoA to the TCA cycle and electrons to produce ATP at the ETC. FAO inhibition, at cellsurface (such as CD36) and mitochondrial-surface (such as CPT1) enzymes, disrupts oxidative metabolism in chemo-resistant leukemia cells, resulting in decreased AML survival, proliferation, clonogenic potential, and engraftment capacity. The bulk normal hematopoietic population did not demonstrate the same dependency on FAO and were less adversely affected (Farge et al., 2017) (Stevens et al., 2020). However, the CD36 and CPT1 pharmacological inhibitors currently under investigation are not approved for clinical use in AML, underscoring the need to identify novel FAO targets.

Secondly, compared to AML cells, normal hematopoietic cells have previously demonstrated a higher glycolytic capacity to compensate for the inhibition of mitochondrial oxidative metabolism. This critical ability to switch substrate oxidation pathways resulted in two divergent metabolic fates, resulting in normal hematopoietic survival and AML cell death (Molina et al., 2018)(Lagadinou et al., 2013)(Sharon et al., 2019). While the majority of AML studies have focused on the inhibition of fat transporters CD36 and CPT1, the intramitochondrial FAO enzymes have not been investigated as therapeutic targets in AML. 
Previously, in subcellular fractionation experiments, the substituents of avo-B localized in the mitochondria, in a CPT1-dependent manner, to suppress FAO-supported mitochondrial respiration (Lee et al., 2015). Taken together with the confirmation of direct FAO inhibition by AYNE in chapter 4, these experiments suggested that AYNE inhibited FAO via an intramitochondrial enzyme target. The following chapter will execute a pharmacodynamic study of VLCAD chemical inhibition by elucidating the mechanism of action and molecular target of AYNE, the anti-AML component of avo-B. 

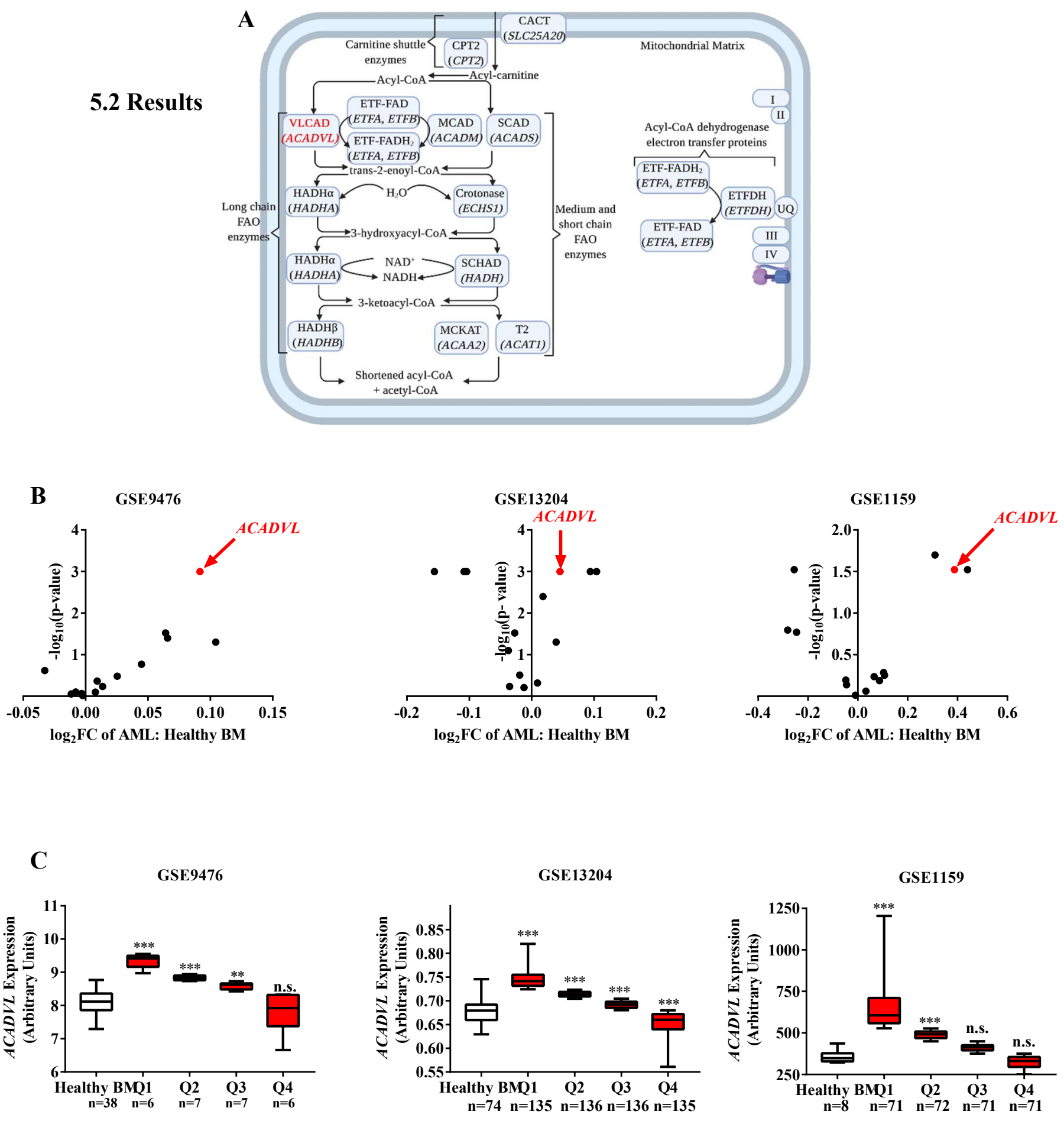

Figure 5.2.1 The Gene Acyl-CoA Dehydrogenase Very Long $(A C A D V L)$ Is Overexpressed In Human AML, Compared To Normal Hematopoiesis

(A) Schematic showing the (FAO) pathway highlighting intramitochondrial enzymes associated with the carnitine shuttle, long, medium, and short chain FAO, and acyl-CoA dehydrogenase (ACAD) electron transfer with the protein VLCAD, coded by the gene $A C A D V L$, highlighted in red. Beneath each protein name (not italicized) are the corresponding gene names (italicized). Each enzyme's full name, short form name, and encoding gene are in Table 1.5.1 (Houten \& Wanders, 2010)(Wanders et al., 2010)(Bartlett \& Eaton, 2004). Abbreviations: I, complex I; II, complex II; III, complex III; IV, complex IV; UQ, ubiquinone. Created in Biorender. (B) Volcano plots 
comparing the fold change in expression of intramitochondrial FAO genes (Figure 5.2.1 A) between the acute myeloid leukemia (AML) patient and normal bone marrow (BM) populations in genomic spatial event (GSE) 9476, GSE13204, and GSE1159 datasets. ACADVL is highlighted in red. Comparisons between genes are presented as a scatter plot with the $\mathrm{x}$-axis as $\log 2$ of the fold change and the y-axis as a negative $\log 10$ of the p-value between AML and normal BM populations. $\mathrm{X}$ and $\mathrm{Y}$ values for all genes across all 3 data sets are tabulated in Table 5.2.1, Table 5.2.2, Table 5.2.3. (C) $A C A D V L$ gene overexpression between AML patient and normal BM populations in GSE9476, GSE13204, and GSE1159. Highlighted in red, AML populations were stratified in 4 quartiles, labelled Q1 through Q4; healthy BM populations are highlighted in white. Statistical analyses for all three datasets are tabulated in Table 5.2.4, Table 5.2.5, Table 5.2.6. Data presented as n.s. not significant; ${ }^{* *} \mathrm{p} \leq 0.002 ; * * * \mathrm{p} \leq 0.001$, using a two-tailed, unpaired Student's $t$-test.

Table 5.2.1 Comparison Of Intramitochondrial FAO Gene Expression In GSE9476

\begin{tabular}{|c|c|c|c|c|c|c|c|}
\hline \multicolumn{8}{|c|}{ Table 5.2.1 - GSE9476 } \\
\hline \multicolumn{8}{|c|}{ GSE9476 (Platform GPL96) } \\
\hline PROBE & GENE & $\underset{\text { value }}{\mathbf{p}}$ & $\begin{array}{l}\text { Mean of } \\
\text { Acute } \\
\text { Myeloid } \\
\text { Leukemia } \\
\text { (AML) }\end{array}$ & $\begin{array}{l}\text { Mean of } \\
\text { Healthy } \\
\text { Bone } \\
\text { Marrow } \\
\text { (BM) }\end{array}$ & $\begin{array}{c}- \\
\log _{10}(p- \\
\text { value })\end{array}$ & $\begin{array}{c}\text { Fold } \\
\text { Change } \\
\text { (FC) }\end{array}$ & $\log _{2} F C$ \\
\hline 203658 at & $S L C 25 A 20$ & 0.24 & 7.24 & 7.4 & 0.61 & 0.97 & -0.03 \\
\hline 204264_at & CPT2 & 0.79 & 5.46 & 5.49 & 0.1 & 0.99 & -0.01 \\
\hline 200710 at & $A C A D V L$ & 0.001 & 8.64 & 8.11 & 3 & 1.06 & 0.09 \\
\hline 202502 at & $A C A D M$ & 0.05 & 7.89 & 7.34 & 1.3 & 1.07 & 0.1 \\
\hline 202366 at & $A C A D S$ & 0.43 & 5.63 & 5.6 & 0.36 & 1.01 & 0.01 \\
\hline $208629 \mathrm{~s}$ at & $H A D H A$ & 0.17 & 9.01 & 8.73 & 0.76 & 1.03 & 0.04 \\
\hline 201007 at & $H A D H B$ & 0.86 & 9.65 & 9.67 & 0.06 & 0.99 & -0.003 \\
\hline 201135 at & ECHS1 & 0.03 & 9.17 & 8.77 & 1.52 & 1.04 & 0.06 \\
\hline 211569 s_at & $H A D H$ & 0.88 & 6.74 & 6.8 & 0.05 & 0.99 & -0.01 \\
\hline 202003 s at & $A C A A 2$ & 0.8 & 7.4 & 7.36 & 0.09 & 1.01 & 0.01 \\
\hline 205412 at & $A C A T 1$ & 0.96 & 8.01 & 8.01 & 0.02 & 0.99 & -0.002 \\
\hline 201931_at & $E T F A$ & 0.33 & 9.24 & 9.08 & 0.48 & 1.02 & 0.03 \\
\hline 202942 at & $E T F B$ & 0.04 & 8.44 & 8.06 & 1.39 & 1.05 & 0.07 \\
\hline 205530 at & ETFDH & 0.58 & 4.47 & 4.43 & 0.23 & 1.01 & 0.01 \\
\hline
\end{tabular}


Table 5.2.2 Comparison Of Intramitochondrial FAO Gene Expression In GSE13204

\begin{tabular}{|c|c|c|c|c|c|c|c|}
\hline \multicolumn{7}{|c|}{ Gable 5.2.2-GSE13204 } \\
\hline \\
\hline
\end{tabular}


Table 5.2.3 Comparison Of Intramitochondrial FAO Gene Expression In GSE1159

\begin{tabular}{|c|c|c|c|c|c|c|c|}
\hline \multicolumn{7}{|c|}{ Table 5.2.3-GSE1159 } \\
\hline \\
\hline PROBE & GENE & $\begin{array}{c}\text { Mean of } \\
\text { Acute } \\
\text { value }\end{array}$ & $\begin{array}{c}\text { Myeloid } \\
\text { Leukemia } \\
\text { (AML) }\end{array}$ & $\begin{array}{c}\text { Mean of } \\
\text { Healthy } \\
\text { Bone } \\
\text { Marrow } \\
\text { (BM) }\end{array}$ & $\begin{array}{c}- \\
\text { log10(p- } \\
\text { value) }\end{array}$ & $\begin{array}{c}\text { Fold } \\
\text { Change } \\
\text { (FC) }\end{array}$ & log2FC \\
\hline 203658_at & $S L C 25 A 20$ & 0.58 & 172.1 & 164.5 & 0.24 & 1.05 & 0.07 \\
\hline 204264 at & $C P T 2$ & 0.56 & 36.07 & 33.49 & 0.25 & 1.08 & 0.11 \\
\hline 200710 at & $A C A D V L$ & 0.03 & 468.7 & 358.1 & 1.52 & 1.31 & 0.39 \\
\hline 202502 at & $A C A D M$ & 0.65 & 205.6 & 193.6 & 0.19 & 1.06 & 0.09 \\
\hline 202366 at & $A C A D S$ & 0.73 & 75.18 & 77.58 & 0.14 & 0.97 & -0.05 \\
\hline 208629 s_at & $H A D H A$ & 0.64 & 518.4 & 536 & 0.19 & 0.97 & -0.05 \\
\hline 201007 at & $H A D H B$ & 0.02 & 635.1 & 512.3 & 1.7 & 1.24 & 0.31 \\
\hline 201135 at & $E C H S 1$ & 0.03 & 281.1 & 207.2 & 1.52 & 1.36 & 0.44 \\
\hline 211569 s_at & $H A D H$ & 0.16 & 96.35 & 117.1 & 0.8 & 0.82 & -0.28 \\
\hline 202003 s_at & $A C A A 2$ & 0.17 & 115.7 & 137.2 & 0.77 & 0.84 & -0.25 \\
\hline 205412 at & $A C A T 1$ & 0.87 & 224.8 & 219.8 & 0.06 & 1.02 & 0.03 \\
\hline 201931 at & $E T F A$ & 0.52 & 241.3 & 224.6 & 0.28 & 1.07 & 0.1 \\
\hline 202942 at & $E T F B$ & 0.97 & 91.1 & 91.73 & 0.01 & 0.99 & -0.01 \\
\hline 205530 at & $E T F D H$ & 0.03 & 45.08 & 53.81 & 1.52 & 0.84 & -0.26 \\
\hline
\end{tabular}


Table 5.2.4 Comparison Of ACADVL Gene Expression Between AML And Normal Samples In GSE9476

\begin{tabular}{|c|c|c|c|}
\hline \multicolumn{4}{|c|}{ Table 5.2.4 - GSE9476 } \\
\hline GSE9476 & $\begin{array}{c}\text { Mean of Healthy } \\
\text { BM }\end{array}$ & $\begin{array}{c}\text { Mean of AML } \\
\text { Quartile }\end{array}$ & $\begin{array}{c}\text { Adjusted P } \\
\text { Value }\end{array}$ \\
\hline Healthy BM vs. Q1 & 8.115 & 9.345 & $<0.001$ \\
\hline Healthy BM vs. Q2 & 8.115 & 8.836 & $<0.001$ \\
\hline Healthy BM vs. Q3 & 8.115 & 8.596 & 0.004 \\
\hline Healthy BM vs. Q4 & 8.115 & 7.794 & 0.11 \\
\hline
\end{tabular}

Table 5.2.5 Comparison Of ACADVL Gene Expression Between AML And Normal Samples In GSE13204

\begin{tabular}{|c|c|c|c|}
\hline \multicolumn{4}{|c|}{ Table 5.2.5 - GSE13204 } \\
\hline GSE13204 & $\begin{array}{c}\text { Mean of Healthy } \\
\text { BM }\end{array}$ & $\begin{array}{c}\text { Mean of AML } \\
\text { Quartile }\end{array}$ & Adjusted P Value \\
\hline Healthy BM vs. Q1 & 0.6792 & 0.7458 & $<0.001$ \\
\hline Healthy BM vs. Q2 & 0.6792 & 0.7137 & $<0.001$ \\
\hline Healthy BM vs. Q3 & 0.6792 & 0.6919 & $<0.001$ \\
\hline Healthy BM vs. Q4 & 0.6792 & 0.6526 & $<0.001$ \\
\hline
\end{tabular}

Table 5.2.6 Comparison Of ACADVL Gene Expression Between AML And Normal Samples In GSE1159

\begin{tabular}{|c|c|c|c|}
\hline \multicolumn{4}{|c|}{ Table 5.2.6 - GSE1159 } \\
\hline GSE1159 & $\begin{array}{c}\text { Mean of Healthy } \\
\text { BM }\end{array}$ & $\begin{array}{c}\text { Mean of AML } \\
\text { Quartile }\end{array}$ & Adjusted P Value \\
\hline Healthy BM vs. Q1 & 358.1 & 653.7 & $<0.001$ \\
\hline Healthy BM vs. Q2 & 358.1 & 490.2 & $<0.001$ \\
\hline Healthy BM vs. Q3 & 358.1 & 412.1 & 0.17 \\
\hline Healthy BM vs. Q4 & 358.1 & 318.6 & 0.43 \\
\hline
\end{tabular}




\subsubsection{The Gene Acyl-CoA Dehydrogenase Very Long $(A C A D V L)$ Is Overexpressed In Human AML, Compared To Normal Hematopoiesis}

Previously, pharmacological inhibition of the cell-surface and mitochondria-surface fat transporters hindered AML mitochondrial metabolism and re-sensitized previously resistant AML cells to current chemotherapies (Farge et al., 2017)(Stevens et al., 2020). Given this critical role of FAO in AML progression and relapse, the gene expression of intra-mitochondrial FAO enzymes (Figure 5.2.1 A, Table 1.5.1) were compared in normal healthy (BM) and AML populations from publicly available databases Genomic Spatial Event (GSE)9476, GSE13204, GSE1159 (Figure 5.2.1 B, Table 5.2.1-3). Across all three datasets, the most consistently overexpressed gene among intramitochondrial FAO enzymes in the AML patient population, compared to the normal healthy BM population, was $A C A D V L$ (Figure 5.2.1 B, Table 5.2.1-3). Within each dataset, the $A C A D V L$ expression was stratified and the AML population was then divided into 4 quartiles. Upon stratification of the three datasets, $A C A D V L$ was overexpressed in 8 out of 12 quartiles across the three datasets (Figure 5.2.1 C, Table 5.2.4-6). 

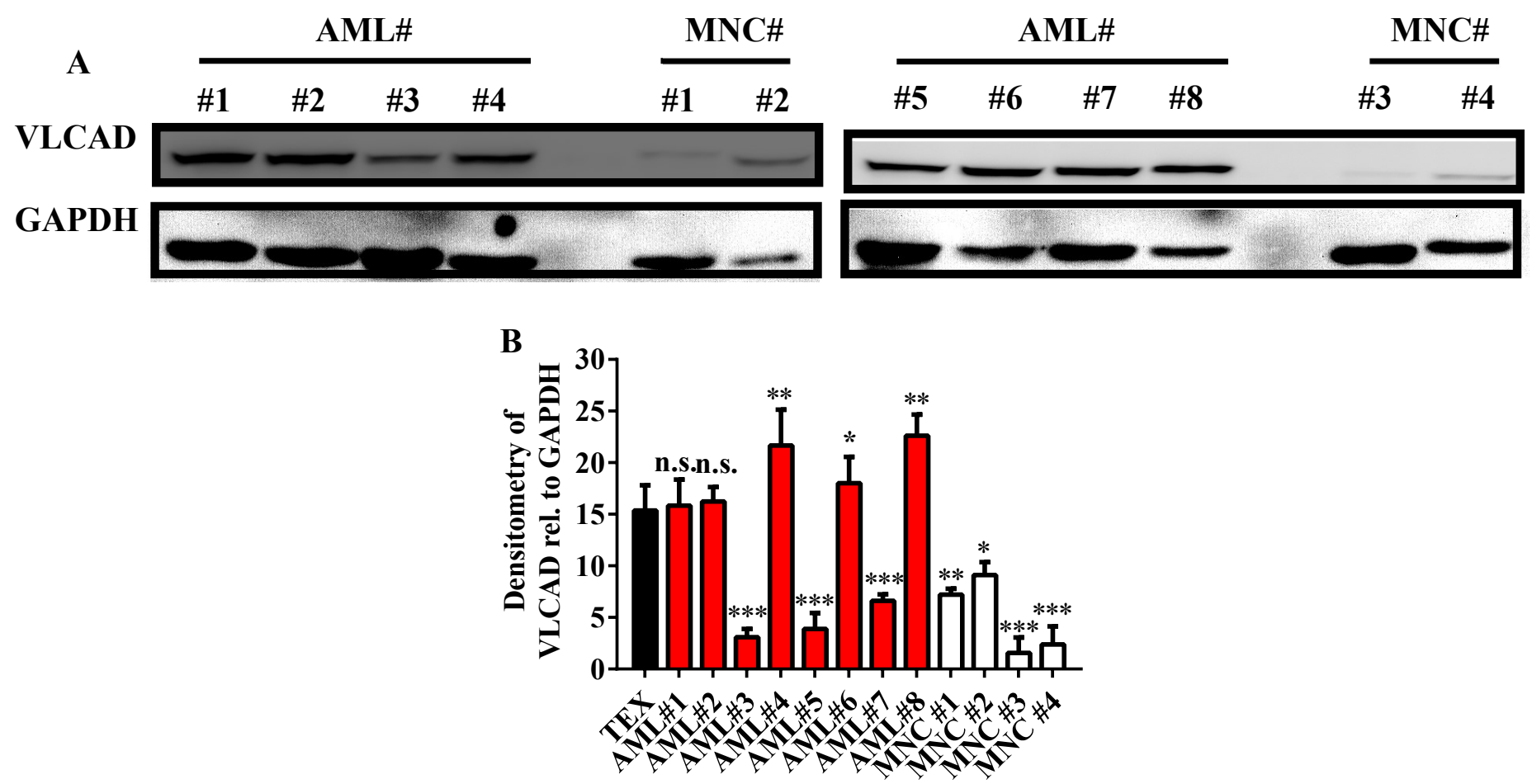

Figure 5.2.2 The very long chain acyl-CoA dehydrogenase (VLCAD) protein is overexpressed in human AML, compared to normal hematopoiesis

(A) Western blot assessing VLCAD protein levels across primary patient-derived AML (AML\#18), isolated from peripheral blood, and normal MNC cells (MNC\#1-4). (B) Comparison of VLCAD protein levels in primary patient-derived AML (Red bars) and MNC samples (White bars) to the AML cell line TEX (Black bar) ( $\mathrm{N}=4$ for TEX, $\mathrm{N}=2$ for primary samples). Individual comparisons are tabulated in Table 5.2.7. For (B), data presented as n.s. not significant; ${ }^{*} \mathrm{p} \leq 0.05$; ** $\mathrm{p} \leq 0.002$; *** $\mathrm{p} \leq 0.001$, using a two-tailed, unpaired Student's $t$-test. See Table 5.2.14 for patient cytogenetics.

Table 5.2.7 Comparing VLCAD expression in primary AML and MNC to TEX

\begin{tabular}{|c|c|c|}
\hline Comparison of VLCAD protein & T value $(\mathbf{d f}=\mathbf{3})$ & P value \\
\hline TEX vs. AML\#1 & $\mathrm{t}=0.1532$ & 0.89 \\
\hline TEX vs. AML\#2 & $\mathrm{t}=1.588$ & 0.21 \\
\hline TEX vs. AML\#3 & $\mathrm{t}=6.635$ & 0.001 \\
\hline TEX vs. AML\#4 & $\mathrm{t}=2.856$ & 0.06 \\
\hline TEX vs. AML\#5 & $\mathrm{t}=5.708$ & 0.001 \\
\hline TEX vs. AML\#6 & $\mathrm{t}=2.546$ & 0.05 \\
\hline TEX vs. AML\#7 & $\mathrm{t}=4.642$ & 0.001 \\
\hline TEX vs. AML\#8 & $\mathrm{t}=4.029$ & 0.03 \\
\hline TEX vs. MNC\#1 & $\mathrm{t}=4.304$ & 0.02 \\
\hline TEX vs. MNC\#2 & $\mathrm{t}=3.022$ & 0.05 \\
\hline TEX vs. MNC\#3 & $\mathrm{t}=7.001$ & 0.001 \\
\hline TEX vs. MNC\#4 & $\mathrm{t}=6.349$ & 0.001 \\
\hline
\end{tabular}




\subsubsection{The Very Long Chain Acyl-CoA Dehydrogenase (VLCAD) Protein Is Overexpressed In Human AML, Compared To Normal Hematopoiesis}

The gene $A C A D V L$ encodes for VLCAD, the intramitochondrial enzyme catalyzing the first of the four step FAO cycle for saturated long chain fats. After determining that the $A C A D V L$ gene is overexpressed in AML (Figure 5.2.1), VLCAD protein levels in primary patient-derived AML cells and MNC samples were quantified by western blot (Figure 5.2.2 A). More than half of the primary patient-derived AML samples (AML\#1, 2, 4, 6, 8) demonstrated comparable or higher VLCAD expression to TEX, a cell line sensitive to AYNE (Figure 5.2.2 B, Table 5.2.7). In contrast, all MNC samples demonstrated significantly lower VLCAD protein levels, compared to TEX (Figure 5.2.2, Table 5.2.7). Taken together, $A C A D V L$ gene expression (Figure 5.2.1) and levels of the encoded protein VLCAD (Figure 5.2.2) were increased in AML, compared to the normal hematopoietic population. 

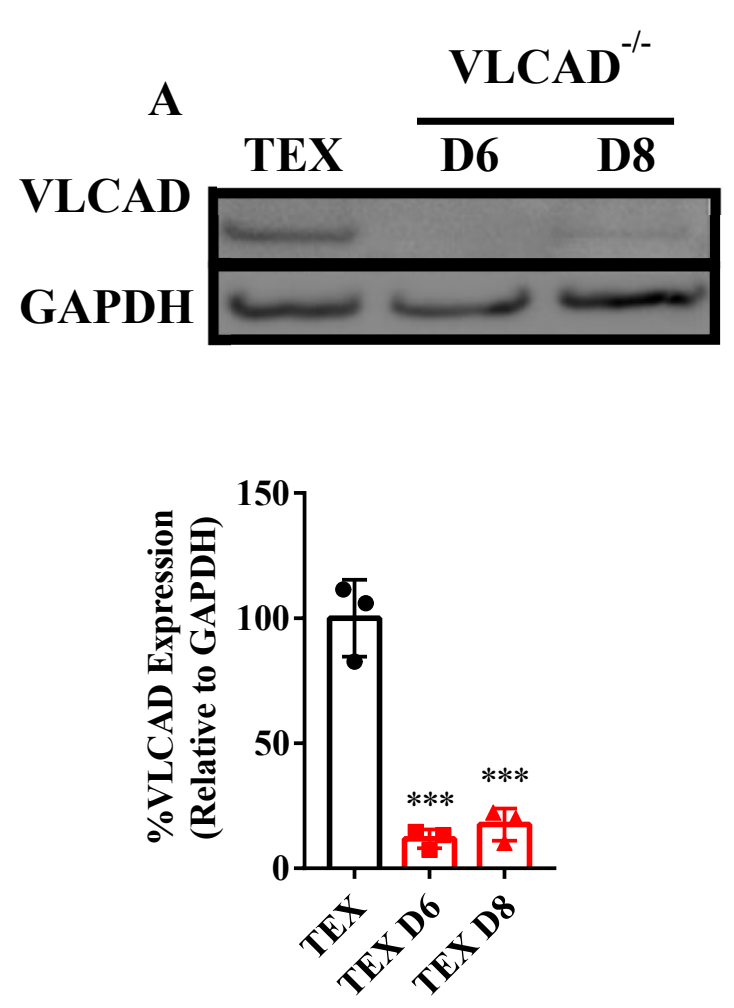

C

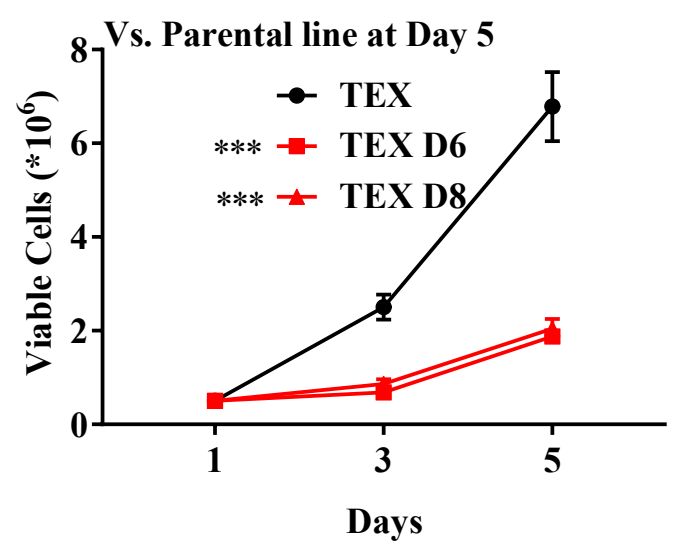

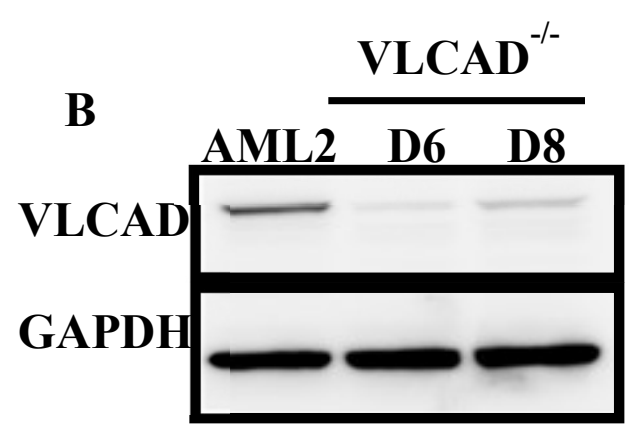

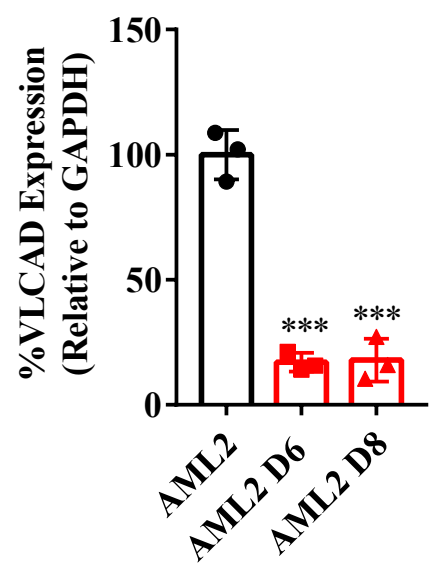

D Vs. Parental line at Day 5
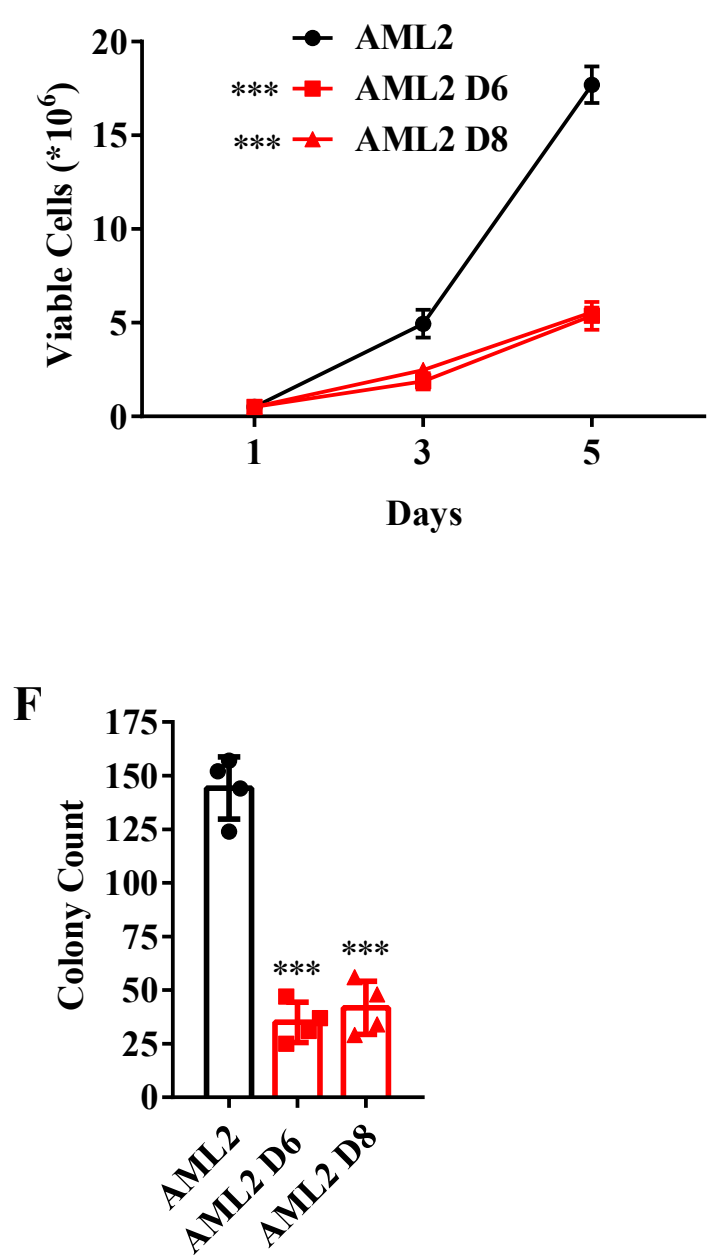
Figure 5.2.3 VLCAD knockdown reduces leukemia proliferation and clonogenic growth Western blot and densitometry confirming VLCAD knockdown in (A) TEX and (B) AML2, where D6 and D8 are plasmids containing VLCAD-targeting shRNA sequences. Proliferation counts after 1, 3, 5 days in (C) TEX (parental TEX compared to the knockdown lines TEX D6 and TEX D8) and (D) AML2 (parental AML2 compared to the knockdown lines AML2 D6 and AML2 D8) $(\mathrm{N}=3$ counts per day). Clonogenic growth after 2 weeks for (E) TEX (parental TEX compared to the knockdown lines TEX D6 and TEX D8) and (F) AML2 (parental AML2 compared to the knockdown lines AML2 D6 and AML2 D8). For (A-F), data presented as mean \pm S.D., n.s. not significant; * $\mathrm{p} \leq 0.05 ; * * \mathrm{p} \leq 0.002 ; * * * \mathrm{p} \leq 0.001$, using a one-way ANOVA, Tukey's post-hoc test. 


\subsubsection{VLCAD Knockdown Reduces Leukemia Proliferation And Clonogenic Growth}

Lentiviral-mediated knockdown of the VLCAD protein was achieved using two shRNA plasmids targeting VLCAD, named D6 and D8, in TEX (Figure 5.2.3 A: F(2,8)=75.16, p $<0.001$; TEX knockdown lines denoted TEX D6 and TEX D8) and AML2 (Figure 5.2.3 B: F(2,8)=110.7, $\mathrm{p}<0.001$; AML2 knockdown lines denoted AML2 D6 and AML2 D8). The near complete VLCAD knockdown caused by the D6 and D8 plasmids significantly reduced cell proliferation in TEX $($ Figure 5.2.3 C: Day 3: $F(2,5)=121.1, p=0.0014$, Day 5: $F(2,5)=480.2, p=0.0002)$ and AML2 (Figure 5.2.3 D: Day 3: $F(2,5)=15.19, p=0.0269), 5$ days $($ Day $5: F(2,5)=370.2, p<0.001)$. VLCAD knockdown also hindered clonogenic potential in TEX (Figure 5.2.3 D: $F(2,11)=89.68$, p < 0.001) and AML2 (Figure 5.2.3 E: $(\mathrm{F}(2,11)=99.1, \mathrm{p}<0.001)$. Taken together, loss of VLCAD in AML cell lines slows proliferation and clonogenic growth. 

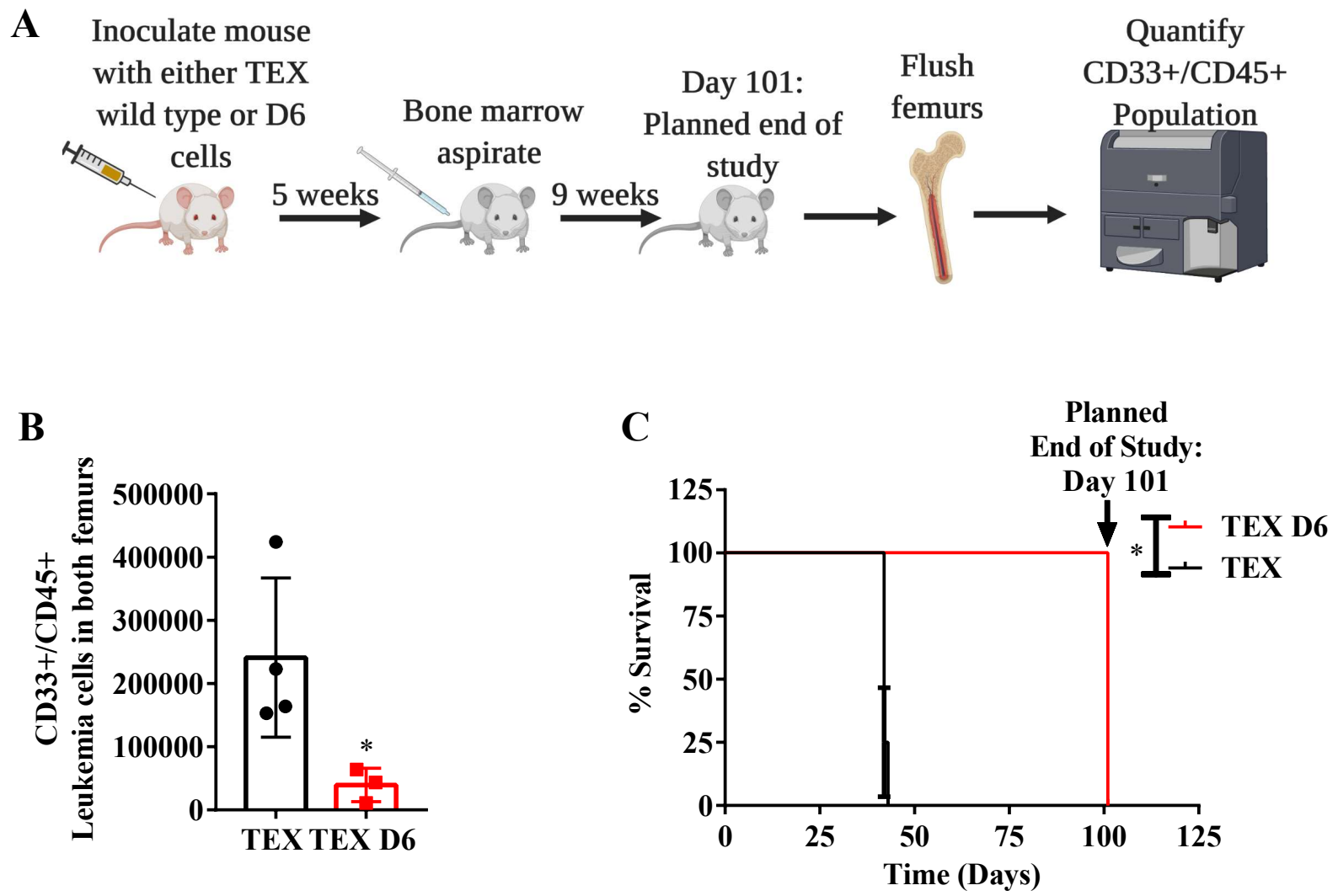

Figure 5.2.4 VLCAD knockdown hinders TEX engraftment

(A) Study schematic highlighting tail vein injection of $1 * 10^{6}$ TEX parental or $1 * 10^{6}$ TEX D6 VLCAD knockdown cells into NSG mice. Engraftment levels were quantified at the planned endpoint at day 101. Abbreviation: CD, cluster of differentiation. Created with Biorender. (B) Engraftment levels of TEX parental and D6 VLCAD knockdown cells in mice femurs at study endpoint assessed by flow cytometry (\%CD33+/CD45+). (C) Overall survival of mice engrafted with either TEX parental or knockdown D6 cells. For (B), data presented as mean \pm S.D., n.s. not significant; * $\mathrm{p} \leq 0.05 ; * * \mathrm{p} \leq 0.002 ; * * * \mathrm{p} \leq 0.001$, using a Mann-Whitney U test. For (C), data presented as mean \pm S.D., $* \mathrm{p}=0.0134$ using a Log-Rank (Mantel-Cox) test. In vivo studies conducted by A. Roma and P. Jayanth. 


\subsubsection{VLCAD Knockdown Hinders TEX Engraftment}

The bone marrow engraftment capacity of TEX D6 knockdown and TEX parental cells was assessed (Figure 5.2.4 A). Injection of an equal number of TEX cells with endogenous or reduced VLCAD protein levels showed that loss of VLCAD significantly hindered leukemia bone marrow engraftment (Figure 5.2.4 B: Mann Whitney $U=0, \mathrm{p}=0.03$ ) and increased survival time (Figure 5.2.4 C: Log-Rank test $p=0.0134)$. Taken together, VLCAD is critical to TEX bone marrow engraftment. 

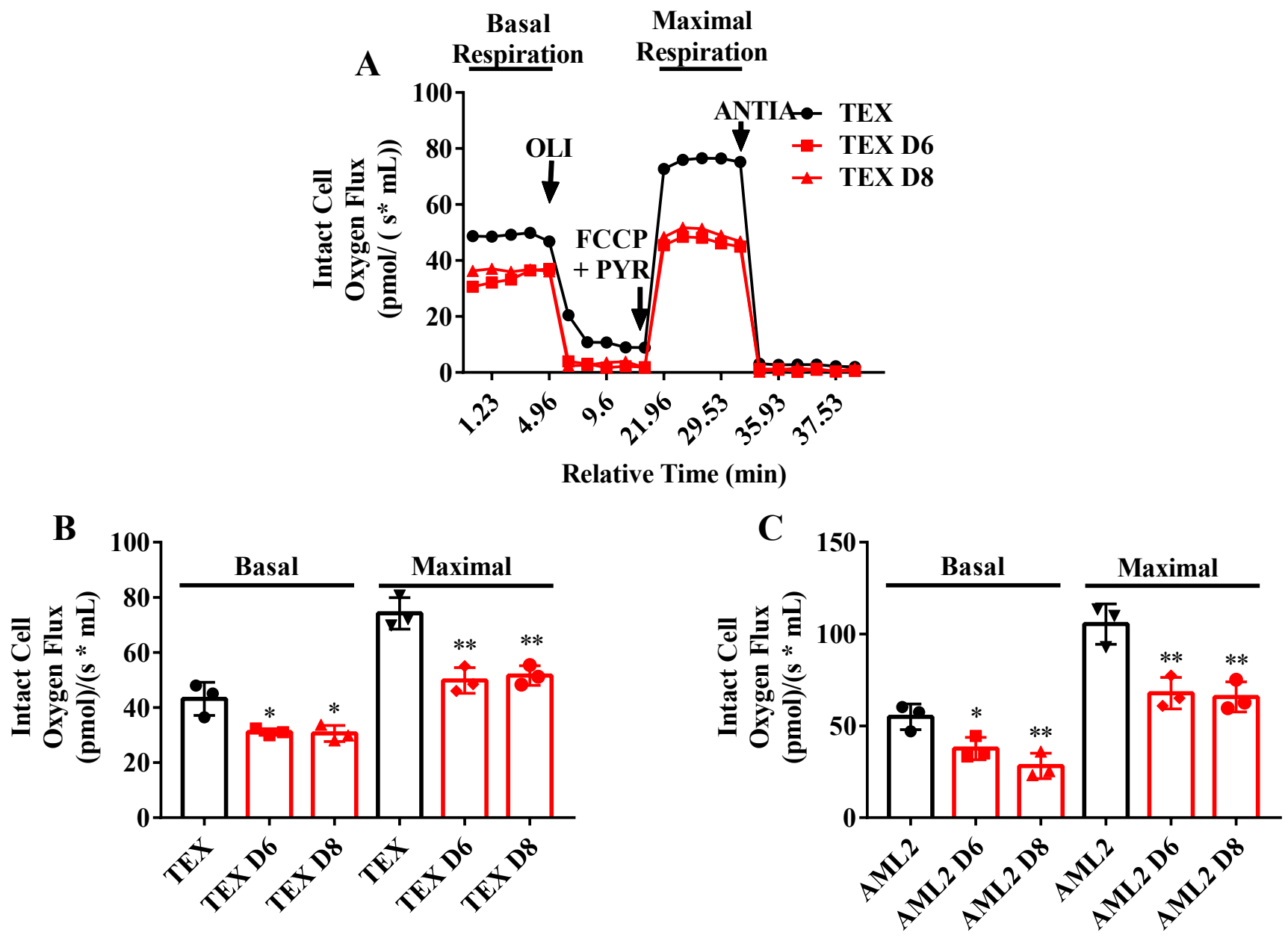

Figure 5.2.5 VLCAD knockdown hinders intact leukemia cell respiration

(A) Schematic showing an example oxygraphy trace quantifying basal and maximal mitochondrial oxygen respiration (van der Windt et al., 2012) of intact TEX cells. Briefly, following injection into the high resolution respirometer (HRR), TEX cells exhibited a basal rate of respiration. The injection of ATP synthase inhibitor oligomycin (OLI) uncoupled ATP synthesis from mitochondrial respiration. Maximal respiration was stimulated with an injection of a chemical uncoupler (FCCP) and pyruvate (PYR). All mitochondrial oxygen consumption was then inhibited with the addition of the complex III inhibitor antimycin A (ANTIA). (B) Intact cell basal and maximal respiration of TEX parental and TEX knockdown (TEX D6 and TEX D8) cell lines. (C) Intact cell basal and maximal respiration of AML2 parental and AML2 knockdown (AML2 D6 and AML2 D8) cell lines. For (B, C), data presented as mean \pm S.D., n.s. not significant; * $p \leq 0.05$; ${ }^{* *} \mathrm{p} \leq 0.002 ;{ }^{* * *} \mathrm{p} \leq 0.001$, using a one-way ANOVA, Tukey's post-hoc test. 


\subsubsection{VLCAD Knockdown Hinders Intact Leukemia Cell Respiration}

The basal respiration of intact cells is fueled by substrates supplied in the incubation media and is tightly coupled to ATP production via oxidative phosphorylation (van der Windt et al., 2012). Maximal respiration is the highest possible rate of respiration following uncoupling of ATP synthesis from oxygen consumption (Figure 5.2.5 A)(van der Windt et al., 2012). VLCAD knockdown hindered the oxygen flux of intact TEX $($ Figure 5.2.5 B: $\operatorname{Basal~} F(2,8)=9.881, \mathrm{p}=0.01$; Maximal $F(2,8)=24.76, p=0.001$ ) and AML2 (Figure 5.2.5 C: Basal: $F(2,8)=12.48, p=0.007$; Maximal: $F(2,8)=17.13, p=0.003)$, confirming that the loss of VLCAD, a long chain FAO enzyme, hindered mitochondrial respiration, in agreement with previous studies demonstrating that FAO supports mitochondrial respiration and OXPHOS (Samudio et al., 2010) (German et al., 2016). 

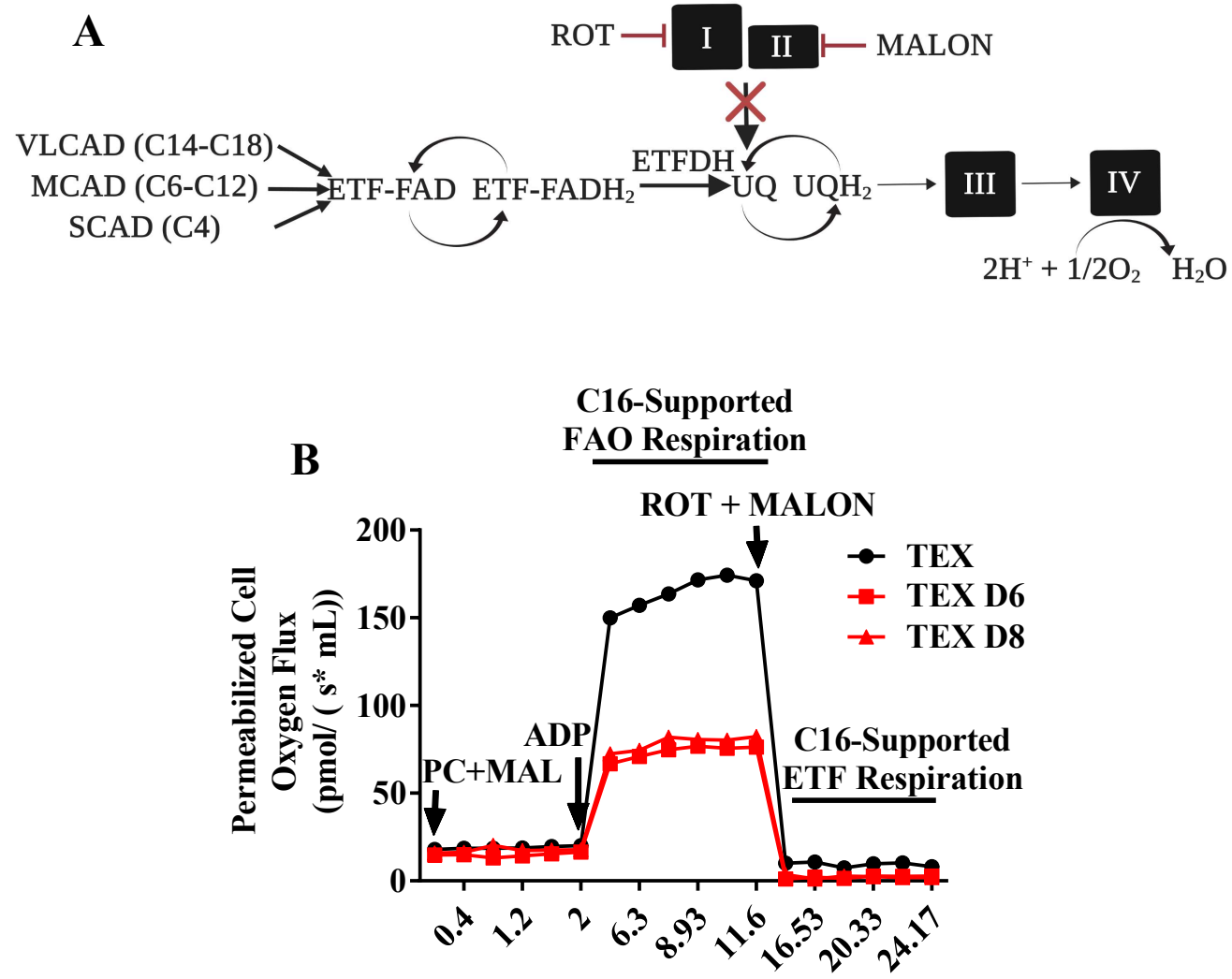

Relative Time (min)
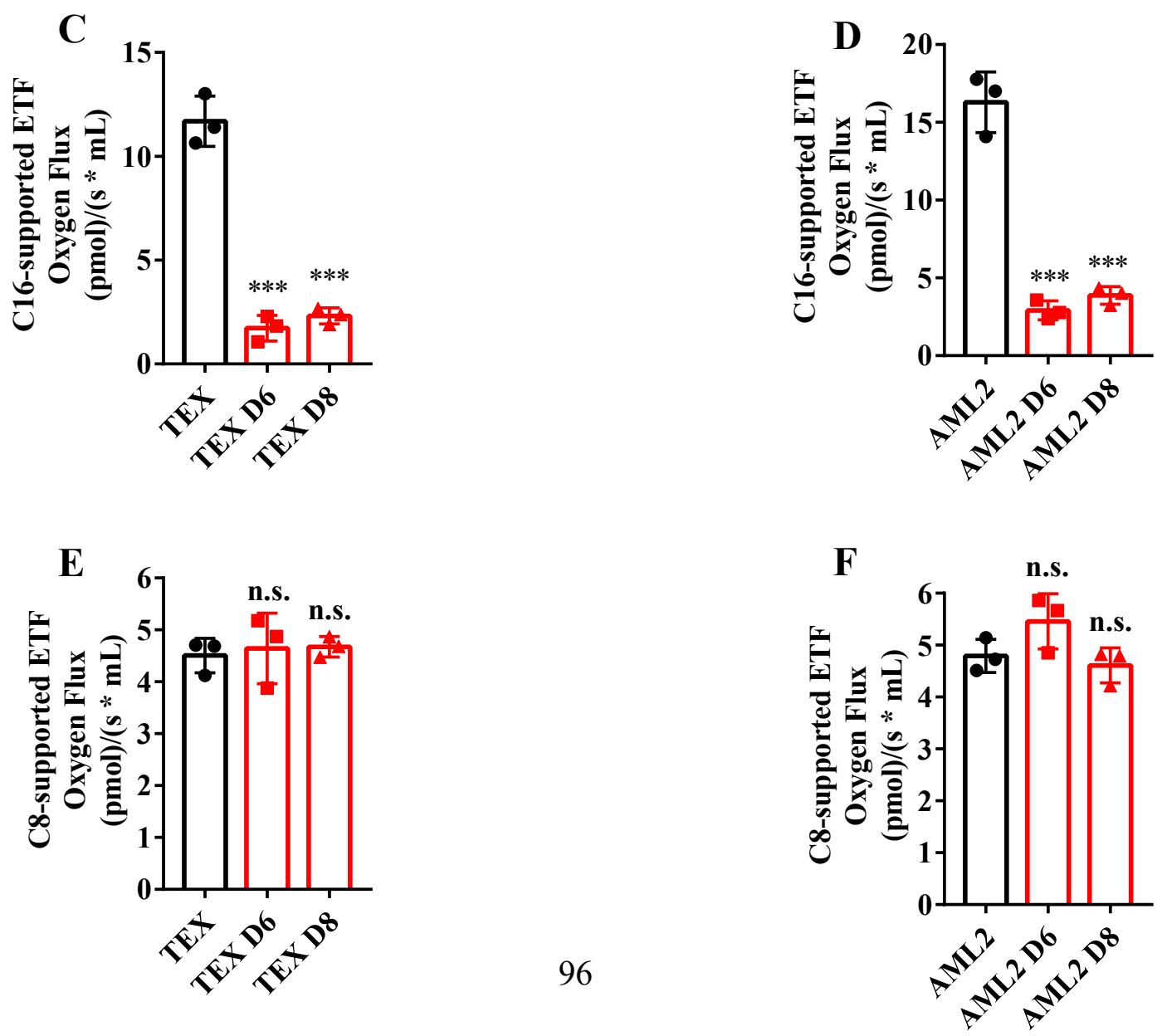
Figure 5.2.6 VLCAD knockdown inhibits C16-supported ETF respiration

(A) Schematic showing electron flow following the oxidation of fats of different carbon lengths at an ACAD enzyme to support OXPHOS (Ojuka et al., 2016). Electrons supplied by VLCAD (for very long fats; 14-18 carbons long), MCAD (for medium length fats; six-12 carbons long), and SCAD (for short length fats; four carbons long) reduce the FAD cofactor attached to the ETF (ETF-FAD). ETF dehydrogenase (ETFDH) oxidizes the reduced ETF-FADH 2 , passing electrons to ubiquinone (UQ) then complex III (III), bypassing complexes I (I) and II (II) (Houten \& Wanders, 2010). Malonate (MALON) and rotenone (ROT) inhibit complexes I (I) and II (II), respectively; residual oxygen flux measured by the respirometer was exclusively respiration supported by electrons passed through the ETF (Ojuka et al., 2016). The remaining ETF-supported respiration was an indirect measure of VLCAD or MCAD activity when palmitoyl-carnitine (PC) or octanoyl-carnitine (OC) is suppled. Created in Biorender. (B) Schematic showing example oxygraph trace quantifying $\mathrm{C} 16$-supported ETF respiration. In brief, permeabilized cells were injected into the respirometer. PC, malate (MAL), and ADP stimulated C16-supported FAO respiration. MALON and ROT inhibited any contribution from complexes I and II, allowing exclusively ETF respiration supported by VLCAD to be assessed. (C) C16-supported ETF respiration of TEX parental, TEX D6, TEX D8 cell lines as an indirect measure of VLCAD activity. (D) C16-supported ETF respiration of AML2 parental, AML2 D6, AML2 D8 cell lines as an indirect measure of VLCAD activity. (E) C8-supported ETF respiration of TEX parental, TEX D6, TEX D8 cell lines as an indirect measure of MCAD activity. (F) C8-supported ETF respiration of AML2 parental, AML2 D6, AML2 D8 cell lines as an indirect measure of MCAD activity. For $(\mathbf{C}-\mathbf{F})$, data presented as mean \pm S.D., n.s. not significant; ${ }^{*} \mathrm{p} \leq 0.05 ; * * \mathrm{p} \leq 0.002 ; * * *$ $\mathrm{p} \leq 0.001$, using a one-way ANOVA, Tukey's post-hoc test. 


\subsubsection{VLCAD Knockdown Inhibits C16-Supported ETF Respiration}

In the mitochondria matrix, the ETF receives electrons from multiple dehydrogenases and shuttles them directly to complex III, bypassing complex I and II (Figure 5.2.6 A)(Houten \& Wanders, 2010). Inhibiting these latter complexes and supplying a 16- or eight-carbon long fat resulted in the exclusive contribution of VLCAD or MCAD to ETF-supported respiration (Figure 5.2.6 B) (Ojuka et al., 2016). VLCAD knockdown decreased C16-supported ETF respiration in TEX (Figure 5.2.6 C: $F(2,8)=141, p<0.001)$ and AML2 (Figure 5.2.6 D: $F(2,8)=112, p<0.001)$. In contrast, respiration supported by MCAD, the ACAD catalyzing the oxidation for medium length fats, was unaffected in both TEX (Figure 5.2.6 E: $F(2,8)=0.1179, p=0.89$ ) and AML2 (Figure 5.2.6 F: $\mathrm{F}(2,8)=3.576, \mathrm{p}=0.09)$. Taken together, VLCAD knockdown exclusively hindered C16-supported ETF respiration, an indirect measure of VLCAD activity, while MCAD activity was unaffected. 

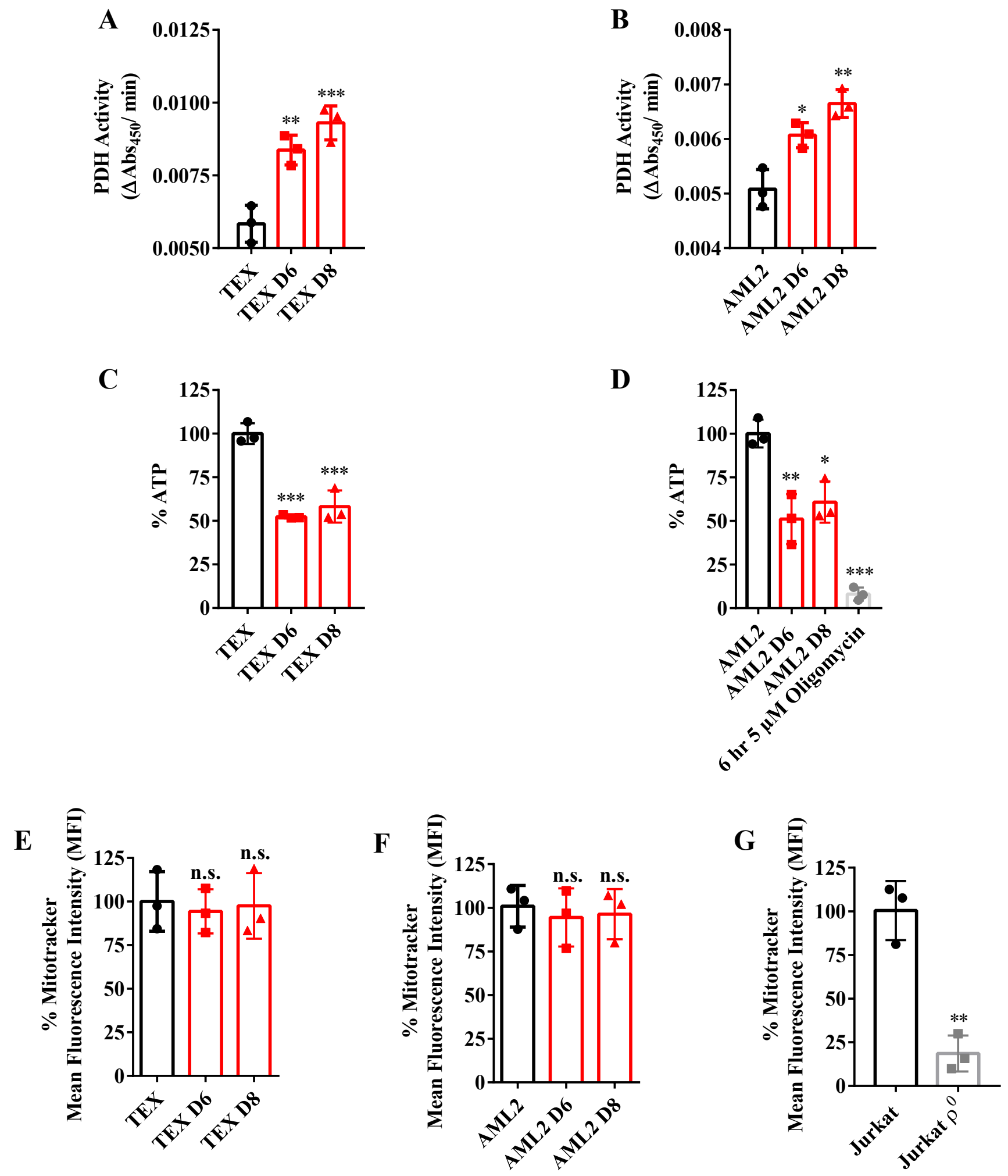

Figure 5.2.7 VLCAD knockdown alters AML mitochondrial metabolism Assessment of pyruvate dehydrogenase (PDH) activity in (A) TEX parental as well as VLCAD knockdown TEX D6 and TEX D8 cell lines and in (B) AML2 parental as well as VLCAD knockdown AML2 D6 and AML2 D8 cell lines. Assessment of ATP levels in (C) TEX parental as well as VLCAD knockdown TEX D6 and TEX D8 cell lines and in (D) AML2 parental as well as VLCAD knockdown AML2 D6 and AML2 D8 cell lines. In a grey-bordered bar, the six hour 
treatment with the ATP synthase inhibitor oligomycin at $5 \mu \mathrm{M}$ is a positive control for ATP depletion (Saito et al., 2015) in AML2. Assessment of mitochondrial mass in (E) TEX parental as well as VLCAD knockdown TEX D6 and TEX D8 cell lines and in (F) AML2 parental as well as VLCAD knockdown AML2 D6 and AML2 D8 cell lines. (G) Assessment of mitochondrial mass in Jurkat and Jurkat $\rho^{0}$. The Jurkat $\rho^{0}$ cell line acts as a positive control for depleted mitochondrial mass (Sriskanthadevan et al., 2015). For (A-F), data presented as mean \pm S.D., n.s. not significant; $* \mathrm{p} \leq 0.05 ; * * \mathrm{p} \leq 0.002 ; * * * \mathrm{p} \leq 0.001$, using a one-way ANOVA, Tukey's post-hoc test. For (G), data presented as n.s. not significant; ** $\mathrm{p} \leq 0.002$, using a two-tailed, unpaired Student's $t$-test. 


\subsubsection{VLCAD Knockdown Alters AML Mitochondrial Metabolism}

To assess the metabolic consequences of VLCAD loss in AML, PDH activity, ATP levels, and mitochondrial mass was assessed. A primary advantage of high rates of FAO is the production of fat-derived acetyl-CoA to the TCA cycle (Martínez-Reyes \& Chandel, 2020)(Sharabi \& Tsokos, 2020). Acetyl-CoA can also be produced by glycolysis through PDH activity (Martínez-Reyes \& Chandel, 2020)(Sharabi \& Tsokos, 2020). PDH activity was increased in both TEX (Figure 5.2.7 A: $\mathrm{F}(2,8)=28.7, \mathrm{p}=0.0008$ ) and AML (Figure 5.2.7 B: $\mathrm{F}(2,8)=22.86 \mathrm{p}=0.0016$ ), suggesting an increased consumption of glucose-derived pyruvate in response to the loss of VLCAD.

In line with previous studies demonstrating that FAO supported AML energy metabolism (German et al., 2016)(Zhang et al., 2020), ATP levels were significantly decreased in TEX (Figure

5.2.7 C: $F(2,8)=50.15, p<0.001$ ) and AML2 (Figure 5.2.7 D: $F(4,12)=40.75, p=0.005$ ). Oligomycin is a potent ATP synthase inhibitor and acts as a positive control for ATP depletion (Saito et al., 2015).

Mitochondrial mass of the knockdown cells was not altered in TEX (Figure 5.2.7 E: $\mathrm{F}(2,8)=0.1281, \mathrm{p}=0.8821)$ or AML2 (Figure 5.2.7 F: $\mathrm{F}(2,8)=0.09151, \mathrm{p}=0.91)$, demonstrating that the observed metabolic changes (e.g., ETF respiration, PDH, and ATP) were not due to a loss of mitochondria numbers. In comparison to the parental Jurkat line, the Jurkat $\rho^{0}$ line acted as a control for mitochondrial mass depletion (Figure 5.2.7 G: $\mathrm{t}(4)=7.16, \mathrm{p}=0.002)$ (Sriskanthadevan et al., 2015). Taken together, VLCAD knockdown triggered a compensatory increase in PDH activity that was insufficient to avert ATP depletion with no significant changes in mitochondrial mass. 


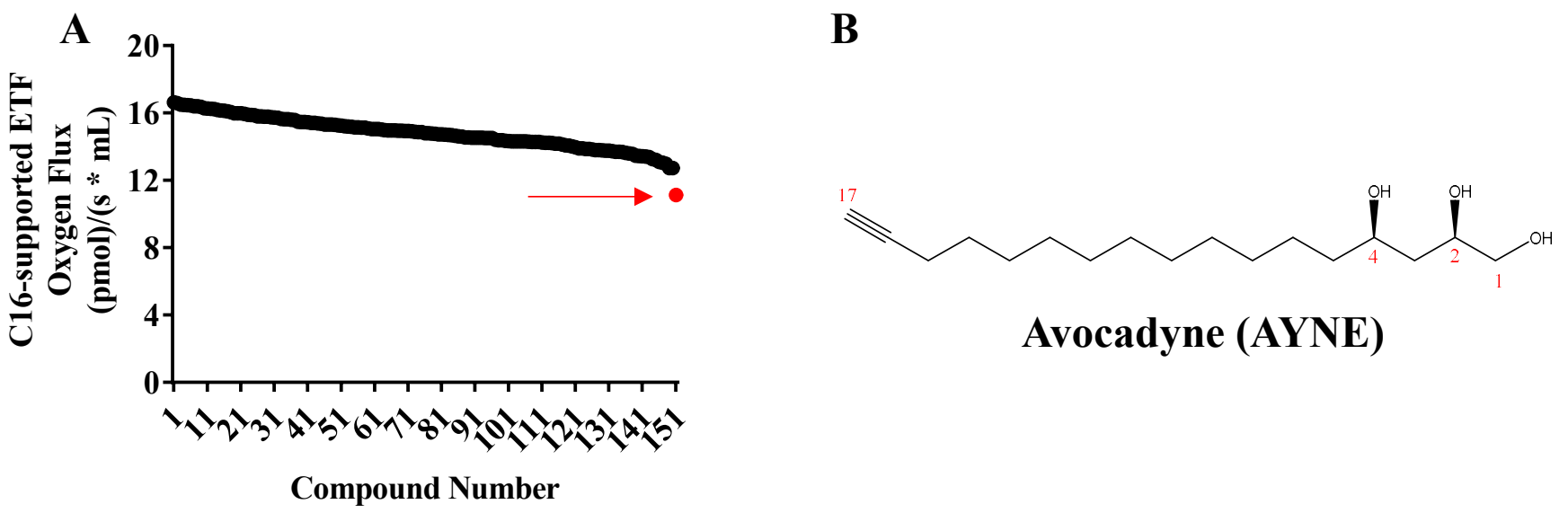

Figure 5.2.8 An unbiased HRR-based screen identifies avocadyne (AYNE) as a VLCAD chemical inhibitor

(A) A HRR screen identified avocadyne (AYNE) as an inhibitor of C16-supported ETF respiration. AML2 cells were incubated with a screen compound $(10 \mu \mathrm{M})$ or a solvent vehicle for one hr and C16-supported ETF respiration was assessed. AYNE (compound 151) is highlighted with a red arrow. (B) Chemical structure of avocadyne (AYNE) highlighting a length of seventeen carbon atoms and hydroxyl groups at the $C-1, C-2, C-4$ positions in red (Kashman et al., 1969)(Sugiyama et al., 1982). Structure drawn in ChemDraw 20.0. 


\subsubsection{An Unbiased High Resolution Respirometry (HRR) Based Screen Identifies Avocadyne (AYNE) As A VLCAD Chemical Inhibitor}

A HRR-based screen assessed the ability of 151 compounds to alter C16-supported ETF respiration of AML2 cells, as an indirect measure of VLCAD activity (Figure 5.2.8 A). AYNE suppressed C16-support ETC respiration. AYNE, a constituent of avo-B, is a 17 carbon long fatty alcohol with a terminal triple bond and hydroxyl groups at the $C-1, C-2, C-4$ positions (Figure 5.2.8 B)(Kashman et al., 1969)(Sugiyama et al., 1982). 
Treat AML2 cells
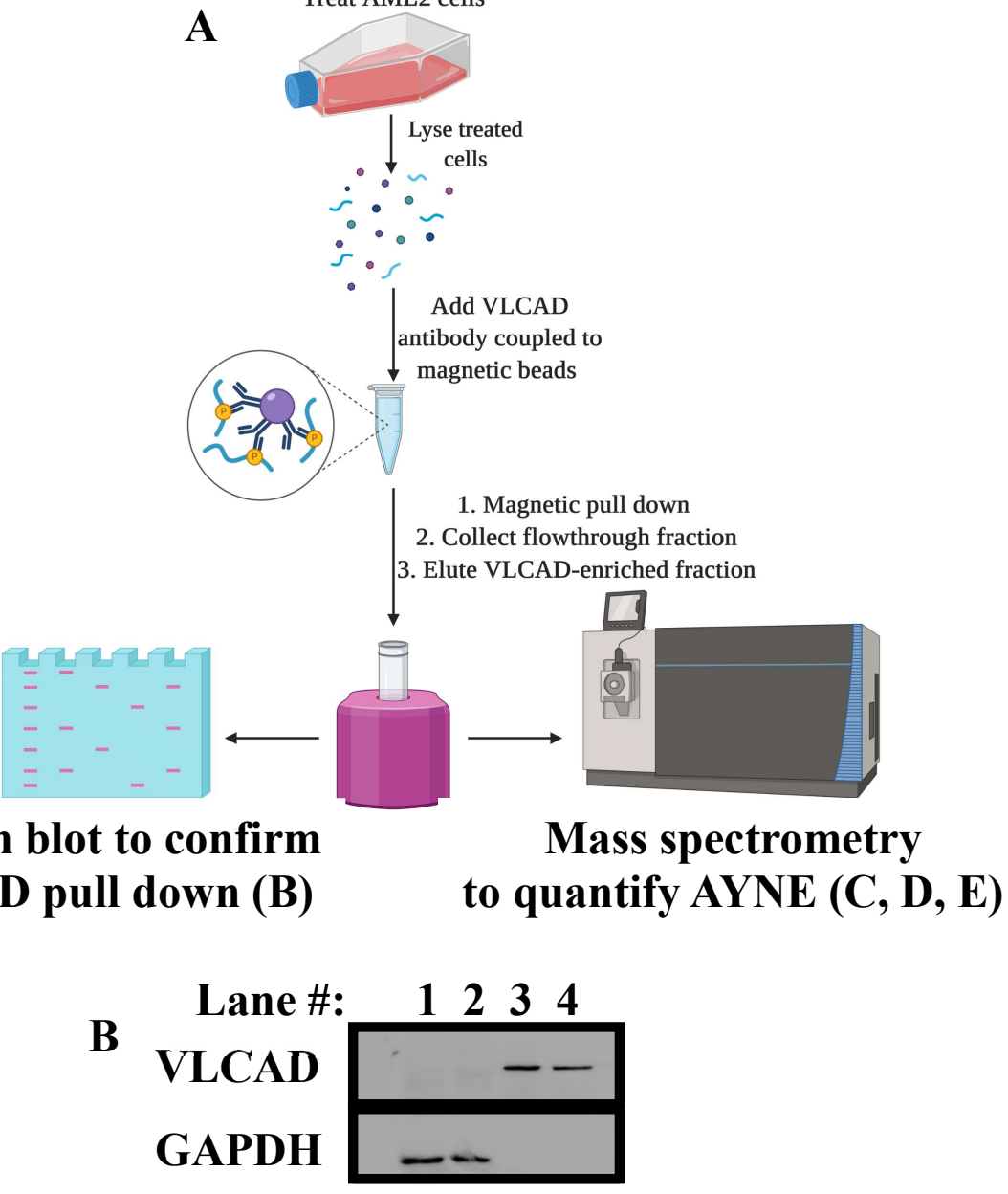

C

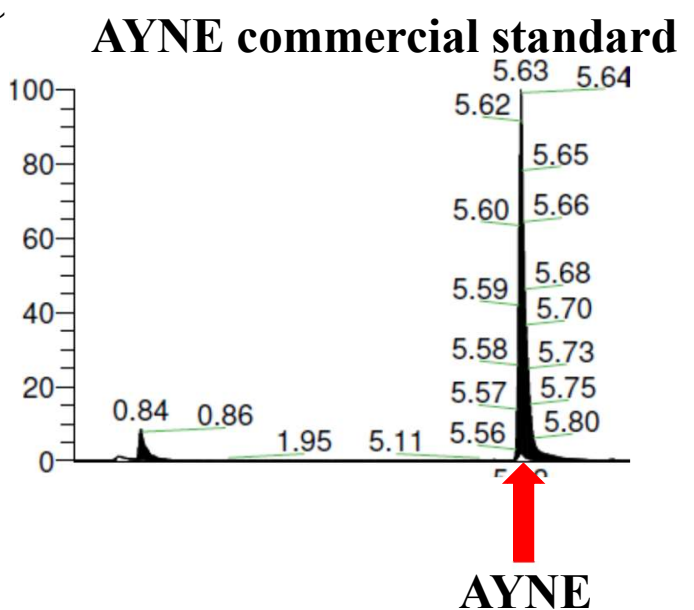

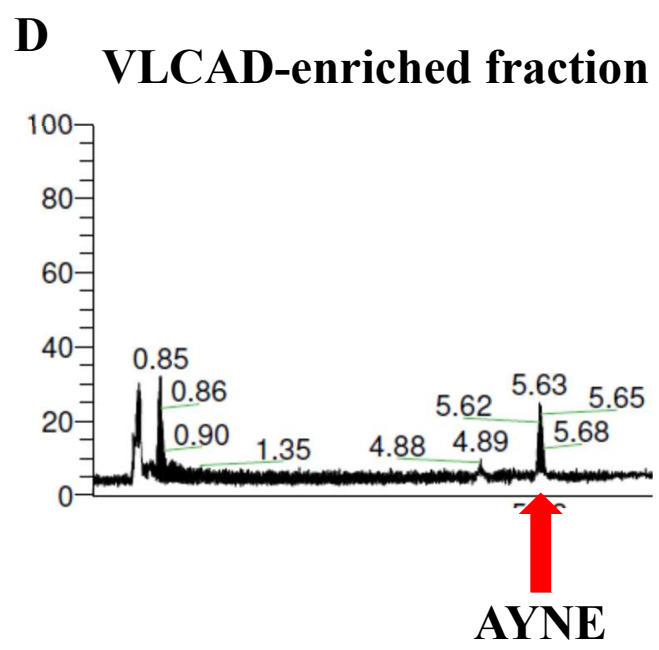

E AYNE extracted from

VLCAD-enriched Co-IP fraction

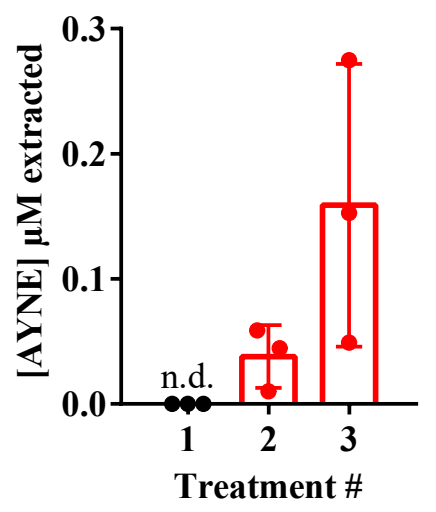


Figure 5.2.9 AYNE precipitates with VLCAD

(A) Experiment schematic demonstrating the pull down of VLCAD in AYNE-treated AML2 cells. Following a three hour AYNE treatment, AML2 cells were lysed and exposed to an anti-VLCAD antibody coupled to magnetic beads. Magnetic separation produces two fractions from the lysate: 1. the flow-through fraction, containing all other cellular components except VLCAD, and 2. the VLCAD-enriched fraction, containing exclusively VLCAD. To determine fraction purity, both fractions underwent immunoblotting to confirm VLCAD pull down. MS analysis of VLCADenriched fractions with a previous published method (Ahmed et al., 2018) was used to quantify AYNE. Abbreviation: VLCAD, very long chain acyl-CoA dehydrogenase. Created in Biorender. (B) Western blot showing VLCAD isolation from the flow-through fractions (lanes \#1 and 2) into VLCAD-enriched fractions (lanes \#3 and 4) following separation by magnetic coimmunoprecipitation (co-IP) $(\mathrm{N}=3)$. Chromatograms showing the detection of AYNE in (C) an AYNE commercial standard and (D) a VLCAD-enriched fraction. The red arrows indicate AYNE elutes at 5.6 minutes. (E) Mass spectrometry (MS) quantification of AYNE in VLCAD-enriched fractions from AYNE-treated AML2 cells ( $N=3$ per treatment). For (E), data presented as mean \pm S.D and tabulated in Table 5.2.8. MS analysis performed by N. Ahmed (University of Guelph Food Science) and Prof. R. W. Smith (University of Waterloo Chemistry).

Table 5.2.8 Quantification of AYNE in VLCAD-enriched fractions

\begin{tabular}{|c|c|c|c|}
\hline $\begin{array}{c}\text { Treatment } \\
\#\end{array}$ & $\begin{array}{c}\text { Antibody } \\
\text { Target }\end{array}$ & $\begin{array}{c}\text { AYNE } \\
\text { Treatment } \\
(\mu \mathrm{M})\end{array}$ & $\begin{array}{c}\text { AYNE } \\
\text { Recovered } \\
(\mathrm{nM})\end{array}$ \\
\hline 1 & No antibody & 10 & n.d. \\
\hline 2 & VLCAD & 10 & $37.50 \pm 26.16$ \\
\hline 3 & VLCAD & 100 & $147.66 \pm 113.77$ \\
\hline
\end{tabular}




\subsubsection{AYNE Precipitates With VLCAD}

To demonstrate that AYNE directly interacted with VLCAD, VLCAD from treated AML2 cells were isolated by co-IP for downstream western blot and MS analysis (Figure 5.2.9 A). Magnetic co-IP produced a flow-through fraction, containing all protein except VLCAD, and a VLCAD-enriched fraction, containing only VLCAD. Fraction purity was confirmed by immunoblotting (Figure 5.2.9 B). VLCAD was isolated in lanes \#3 and \#4 from the flow-through fraction in lanes \#1 and \#2. The absence of the VLCAD band in lanes \#1 and \#2 indicated a complete separation of VLCAD protein from the flowthrough fraction (Figure 5.2.9 B). In contrast, the lack of glyceraldehyde 3-phosphate dehydrogenase (GAPDH), as a loading control, suggested purity of the VLCAD fraction (Figure 5.2.9 B). Detection of AYNE in a commercial standard, using a previously published LC/MS method (Ahmed et al., 2018), exhibited an elution time of 5.6 minutes (Figure 5.2.9 C). Following co-IP, MS analysis of the VLCAD-enriched fraction revealed a peak at 5.6 minutes, confirming the presence of AYNE in the VLCAD-enriched fraction (Figure 5.2.9 D). AML2 were subjected to three different treatments to demonstrate a physical interaction with VLCAD (Figure 5.2.9 E). In treatment \#1, AML2 cells received a 10 $\mu \mathrm{M}$ AYNE treatment for three hours but were exposed to magnetic beads lacking an anti-VLCAD antibody following cell lysis. In treatment \#2 and \#3, AML2 cells received either a 10 or $100 \mu \mathrm{M}$ AYNE treatment for three hours and were exposed to an anti-VLCAD antibody coupled to magnetic beads following cell lysis. The lack of AYNE detected in the absence of an anti-VLCAD antibody confirmed a lack of non-specific binding between AYNE and the magnetic bead. Following AYNE treatment and exposure to the anti-VLCAD antibody, AYNE was recovered in VLCAD-enriched fractions at both concentrations (Table 5.2.8). Taken together, AYNE binds to and inhibits VLCAD activity through a direct physical interaction. 
A

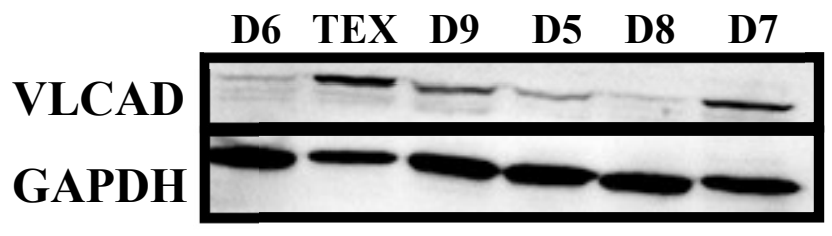

VLCAD Knockdown: TEX

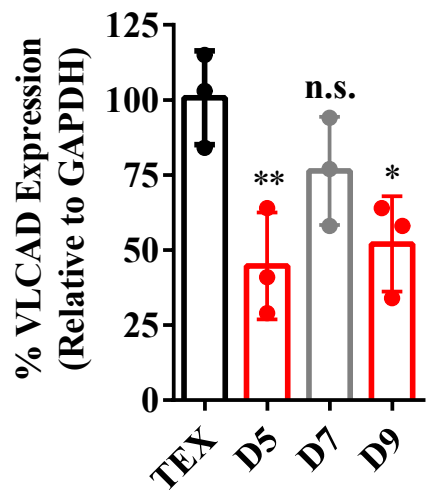

B

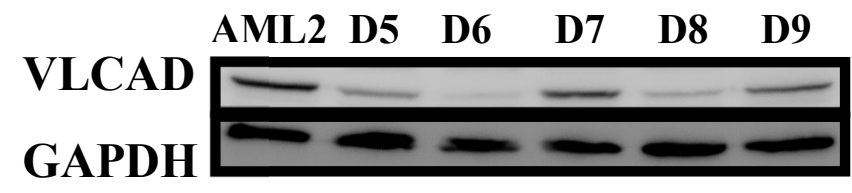

VLCAD Knockdown: AML2

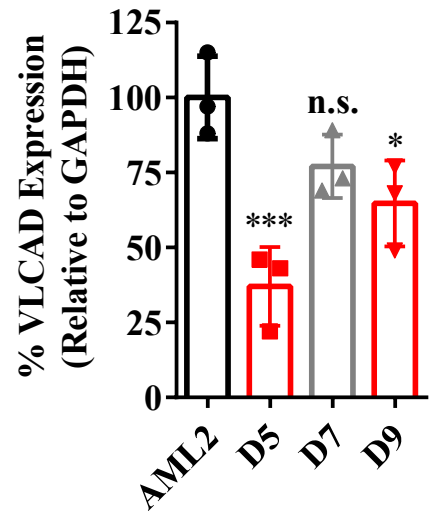

C TEX proliferation upon VLCAD knockdown

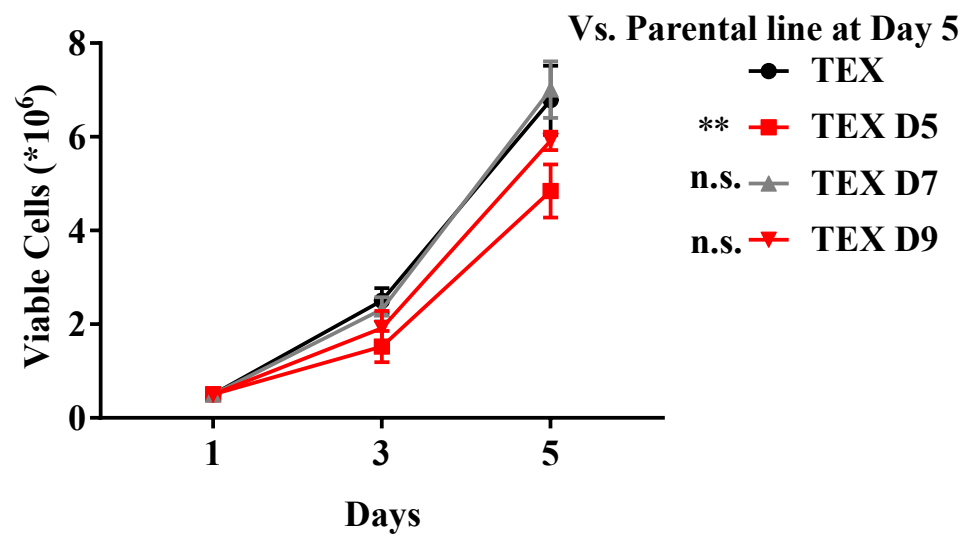

D AML2 proliferation upon VLCAD knockdown

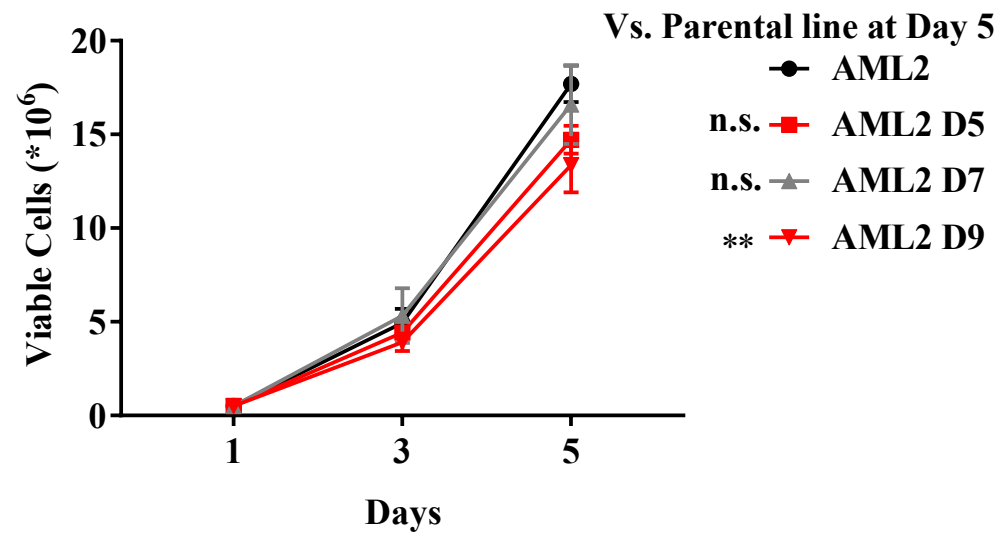


Figure 5.2.10 Partial VLCAD knockdown did not significantly reduce AML proliferation to the same extent as a complete VLCAD knockdown

Western blot and densitometry demonstrating partial VLCAD knockdown in (A) TEX and (B) AML2, where D5, D6, D7, D8, D9 are knockdown constructs. Proliferation counts after 1, 3, 5 days in (C) TEX (parental TEX compared to the knockdown lines TEX D5, TEX D7, TEX D9) and (D) AML2 (parental AML2 compared to the knockdown lines AML2 D5, AML2 D7, AML2 D9). For (A-D), experiments repeated in triplicate. For $(\mathbf{C}, \mathbf{D})$, data presented as mean \pm S.D., n.s. not significant; ${ }^{*} \mathrm{p} \leq 0.05 ; * * \mathrm{p} \leq 0.002 ; * * * \mathrm{p} \leq 0.001$, using a one-way ANOVA, Tukey's post-hoc test. 


\subsubsection{Partial VLCAD Knockdown Does Not Consistently Reduce AML Proliferation}

Previously, VLCAD expression was shown to be varied across the AML patient population

(Figure 5.2.2). To demonstrate that AYNE could inhibit ETF-supported respiration in cells with varied levels of endogenous VLCAD, TEX and AML2 cell lines with a partial knockdown of VLCAD were created. The short hairpin plasmids D5 and D9 achieved significant knockdown of VLCAD across both TEX (Figure 5.2.10 A: $\mathrm{F}(3,11)=6.82, \mathrm{p}=0.01$ ) and AML2 (Figure 5.2.10 B: $\mathrm{F}(3,11)=12.22, \mathrm{p}=0.002)$. Compared to the TEX parental line, partial VLCAD knockdown resulted in significantly reduced proliferation with the D5 plasmid but not with the D7 or D9 plasmids after 5 days (Figure 5.2.10 C: $\mathrm{F}(3,11)=9.131$ ). In contrast, partial VLCAD knockdown in AML2 reduced proliferation with the D9 plasmid after 5 days (Figure 5.2.10 D: F(3,11)=7.147, $\mathrm{p}=0.01$ ). Taken together with previously presented data on D6 and D8 (Figure 5.2.3), a near complete knockdown of VLCAD is required to hinder AML proliferation. 
A VLCAD Knockdown: TEX Oxygen Flux
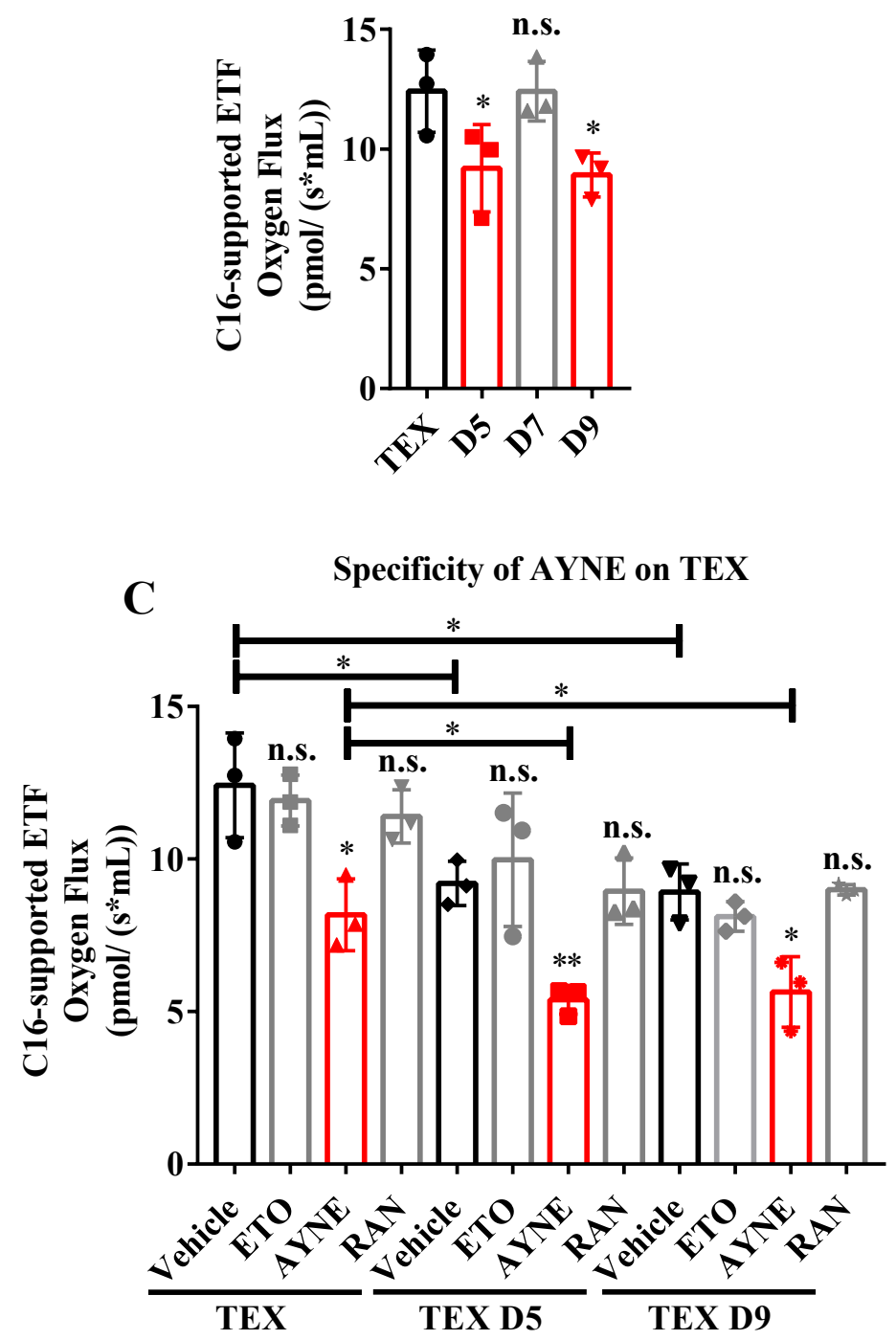

B VLCAD Knockdown: AML2 Oxygen Flux

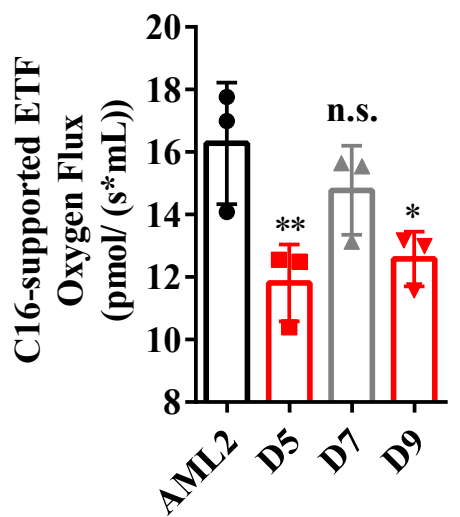

$\mathbf{D}$

Specificity of AYNE on AML2

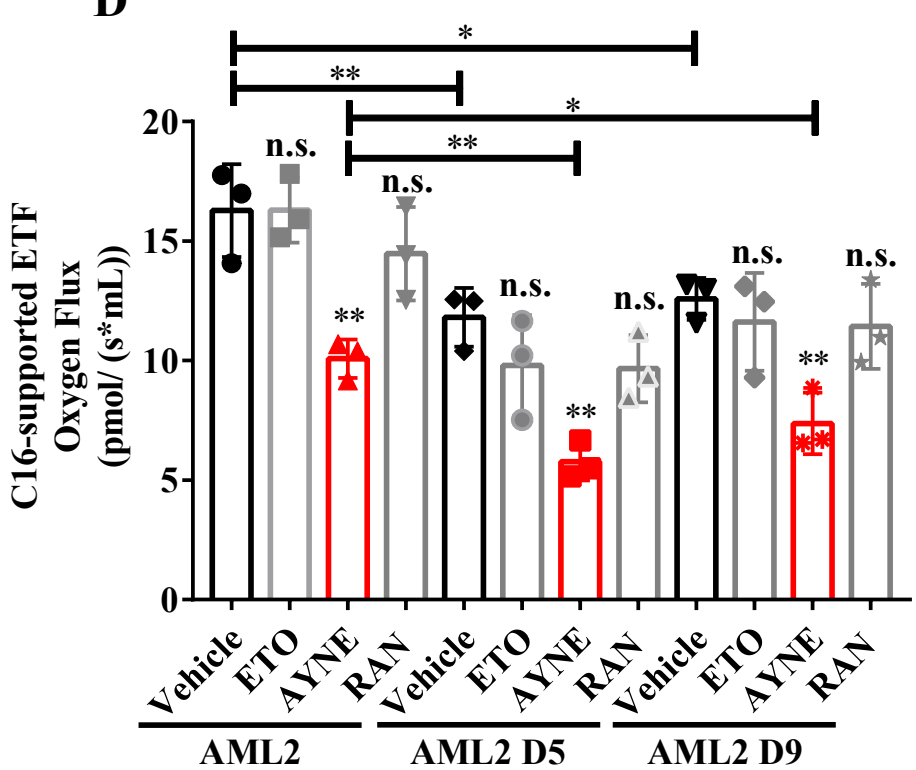

Figure 5.2.11 C16-supported ETF respiration is exclusively hindered by AYNE, but not ETO and RAN

Assessment of C16-supported ETF respiration following partial VLCAD knockdown in (A) TEX and (B) AML2. Assessment of C16-supported ETF respiration of partial VLCAD knockdown lines after a one-hour treatment with either a solvent vehicle, $100 \mu \mathrm{M}$ ETO, $1.5 \mathrm{mM}$ ranolazine (RAN), or $10 \mu \mathrm{M}$ AYNE in (C) TEX and (D) AML2. For (A - D), data presented as mean \pm S.D., n.s. not significant; * $\mathrm{p} \leq 0.05 ; * * \mathrm{p} \leq 0.002 ; * * * \mathrm{p} \leq 0.001$, using a one-way ANOVA, Tukey's post-hoc test. 


\subsubsection{C16-supported ETF Respiration Is Exclusively Hindered By AYNE, But Not ETO And RAN}

Partial VLCAD knockdown coincided with reduced C16-supported ETF respiration in D5 and D9 but not D7 in TEX (Figure 5.2.11 A: $F(5,17)=44.19, \mathrm{p}<0.001)$ and AML2 (Figure 5.2.11 B: $F(5,17)=65.36, p<0.001)$. Knockdown lines that received the D7 plasmid did not exhibit a significant decrease in VLCAD protein (Figure 5.2.10 A, B); this corresponded with no significant change in C16-supported ETF respiration (Figure 5.2.11 A, B).

C16-supported ETF respiration is an indirect measure of VLCAD activity; inhibitors of other FAO enzymes, such as RAN and ETO, are not expected to alter C16-supported ETF respiration as they are not VLCAD inhibitors (Samudio et al., 2010). To further demonstrate the specificity of AYNE to VLCAD inhibition, TEX and AML2 cell lines exhibiting partial VLCAD knockdown were treated with AYNE, ETO, and RAN. Consistent with specificity for VLCAD, AYNE decreased C16-supported ETF respiration in TEX (Figure 5.2.11 C: TEX +AYNE vs TEX D5 + AYNE vs TEX D9 + AYNE: $F(2,8)=14.53, p=0.005$ ) and AML2 (Figure 5.2.11 D: AML2+AYNE vs AML2 D5+AYNE vs AML2 D9 + AYNE: $\mathrm{F}(2,8)=6.195, \mathrm{p}=0.02)$.

In contrast, FAO inhibitors that do not target VLCAD (e.g., ETO and RAN) had no effect on C16-supported ETF respiration as they do not target ACAD enzymes that transfer elections through the ETF to the ETC. ETO targets CPT1 (Samudio et al., 2010) and did not further reduce C16-supported ETF respiration in TEX (Figure 5.2.11 C: TEX +ETO vs TEX D5 + ETO vs TEX D9 + ETO: $F(2,8)=34.18, p=0.09$ ) and AML2 (Figure 5.2.11 D: AML2+ETO vs AML2 D5+ETO vs AML2 D9 + ETO: $\mathrm{F}(2,8)=9.43, \mathrm{p}=0.14)$. Similarly, RAN targets 3-ketoacyl thiolase activity of the MTP $\beta$ subunit (Samudio et al., 2010) and also did not further reduce C16-supported ETF respiration in TEX (Figure 5.2.11 C: TEX +RAN vs TEX D5 + RAN vs TEX D9 + RAN: 
$\mathrm{F}(2,8)=26.41, \mathrm{p}=0.10)$ and AML2 (Figure 5.2.11 D: AML2+ETO vs AML2 D5+ETO vs AML2 D9 + ETO: $F(2,8)=45.16, p=0.17)$. Taken together, AYNE is a FAO inhibitor that specifically targets VLCAD. Figure 5.2.2 noted that VLCAD expression was varied within AML patient samples; the inhibition of C16-supported ETF respiration in AML cell lines with a partial VLCAD knockdown suggests that AYNE is capable of inhibiting leukemia cells with varied VLCAD expression. 
A

TEX

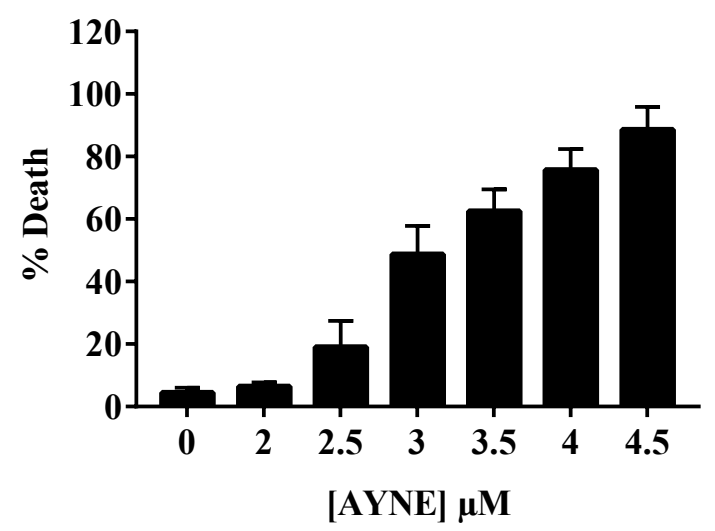

B

AML2

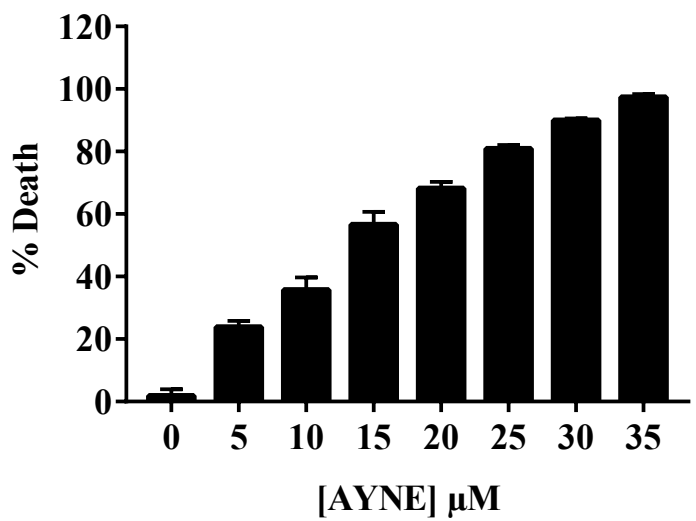

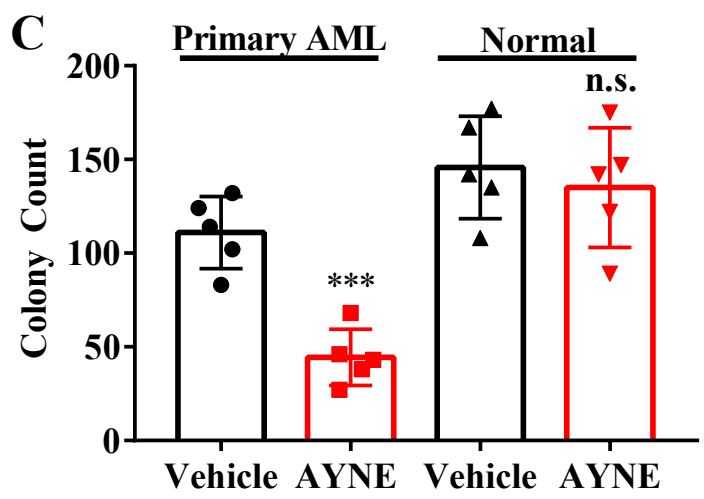

Figure 5.2.12 AYNE kills TEX and AML2 while suppressing primary patient-derived AML clonogenic growth

Death of (A) TEX and (B) AML2 cells following a $72 \mathrm{hr}$ AYNE treatment. Cell death assessed by flow cytometry with a 7AAD stain $(\mathrm{N}=3$ ). (C) Clonogenic growth of primary AML cells (AML\#9 - AML\#13) and normal MNCs ( $\mathrm{n}=5$ ) following a two-week treatment with either solvent vehicle or $10 \mu \mathrm{M}$ AYNE. For (A, B), IC 50 values for TEX and AML2 were calculated using dose response - inhibition equation on GraphPad Prism 7.0. For (C), data presented as mean \pm S.D., n.s. not significant; ${ }^{*} \mathrm{p} \leq 0.05 ; * * \mathrm{p} \leq 0.002 ;{ }^{* * *} \mathrm{p} \leq 0.001$, using a two-tailed, unpaired Student's $t$-test. See Table 5.2.14 for patient cytogenetics. 


\subsubsection{AYNE Kills TEX And AML2 While Suppressing Primary AML Clonogenic Growth}

Having demonstrated that AYNE binds to VLCAD, AYNE's ability to kill AML cell lines and suppress clonogenic growth was assessed. AYNE induced dose-dependent cell death in TEX (Figure 5.2.12 A: $3.10 \pm 0.12 \mu \mathrm{M}$ ) and AML2 (Figure 5.2.12 B: $11.39 \pm 0.90 \mu \mathrm{M}$ ). Clonogenic growth, a measure of long term proliferation and differentiation potential, was suppressed in primary AML (Figure 5.2.12 C: $\mathfrak{t}(4)=8.871, p<0.001$ ) but was unaffected in normal donorderived samples (Figure 5.2.12 C: $\mathrm{t}(4)=1.26, \mathrm{p}=0.25$ ). Taken together, VLCAD inhibition induced AML death and suppressed clonogenic growth, while sparing hematopoietic colony formation. 
A

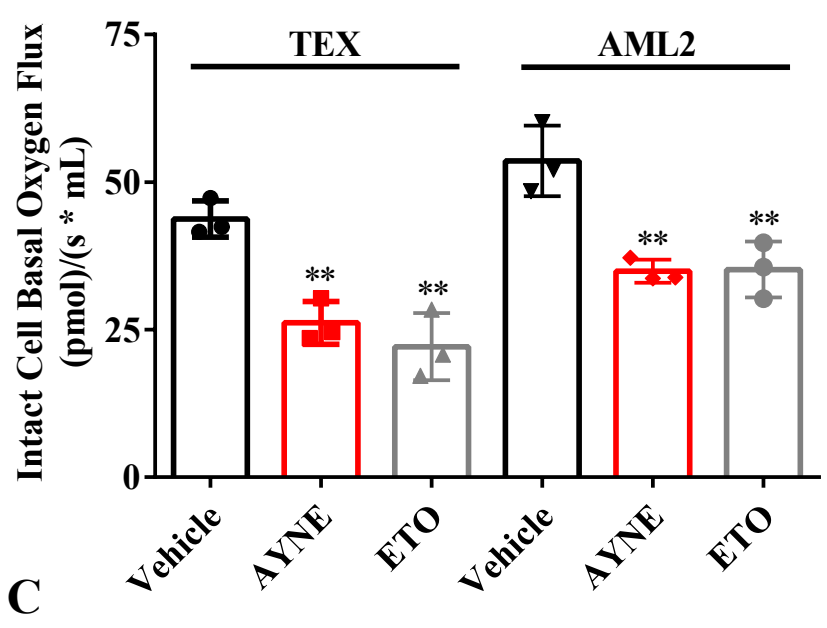

TEX

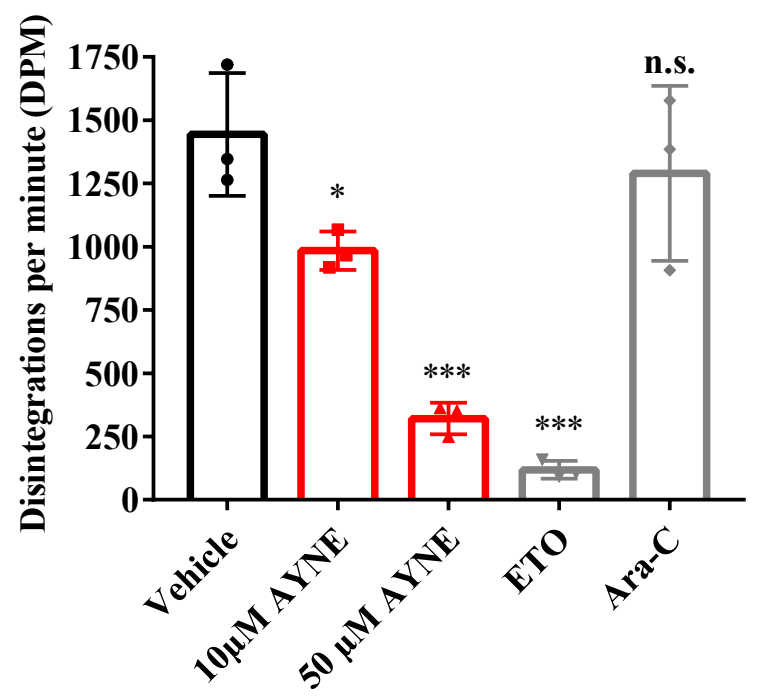

B

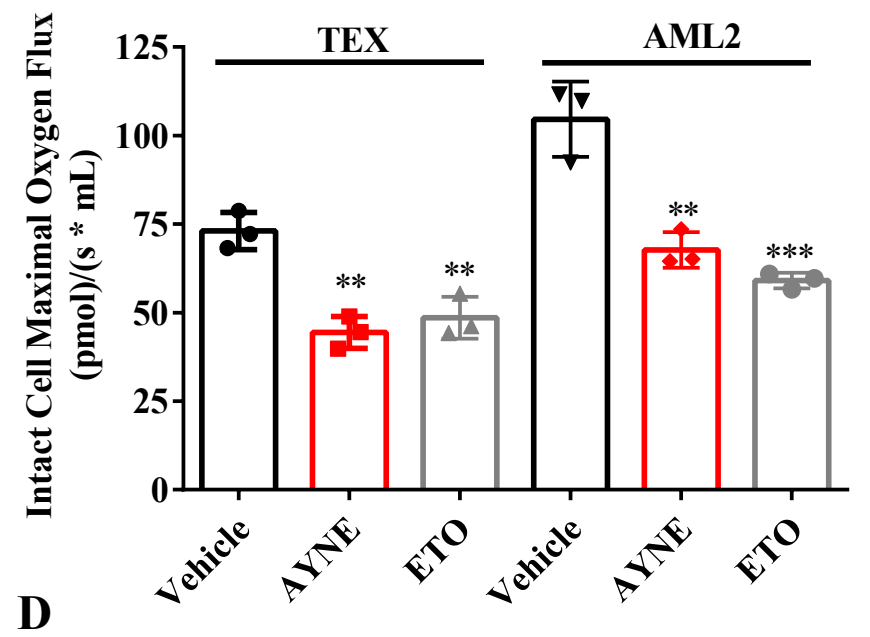

AML2

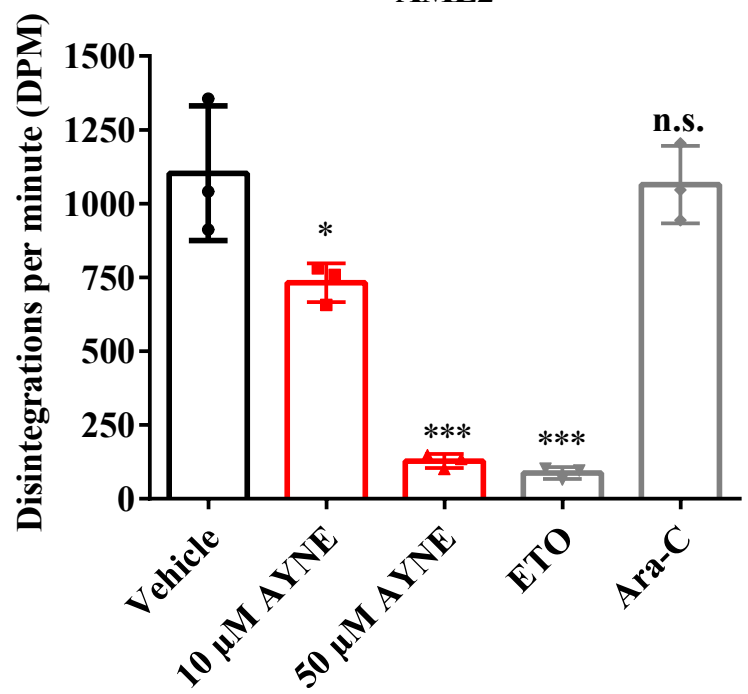

Figure 5.2.13 AYNE directly inhibits mitochondrial long chain FAO to hinder mitochondrial respiration in intact leukemia cells

Intact leukemia cell (A) basal and (B) maximal respiration following a one-hour treatment with a solvent vehicle or $10 \mu \mathrm{M}$ AYNE or $100 \mu \mathrm{M}$ etomoxir (ETO), (positive control for FAO inhibition) (Samudio et al., 2010). Rates of complete FAO, using radiopalmitate, in AML cell lines (C) TEX and (D) AML2 following a three-hour treatment with a solvent vehicle or AYNE (at 10 or $50 \mu \mathrm{M}$ ), or $100 \mu \mathrm{M} \mathrm{ETO}$, or $1 \mu \mathrm{M}$ cytarabine (Ara-C, negative control for FAO inhibition). For (A-D), data presented as mean \pm S.D., n.s. not significant; * $\mathrm{p} \leq 0.05$; ** $\mathrm{p} \leq 0.002$; *** $\mathrm{p} \leq 0.001$, using a one-way ANOVA, Tukey's post-hoc test. For $(\mathbf{C}, \mathbf{D})$, experiments were performed by K. Rea and Prof. T. A. Akhtar (University of Guelph Molecular and Cellular Biology). 


\subsubsection{AYNE Directly Inhibits Mitochondrial Long Chain FAO To Hinder \\ Mitochondrial Respiration In Intact Leukemia Cells}

FAO inhibition by AYNE suppressed basal and maximal mitochondrial respiration of intact TEX (Figure 5.2.13 A: Basal: $\mathrm{F}(2,8)=21.53, \mathrm{p}=0.002$; Figure 5.2.13 B: Maximal: $\mathrm{F}(2,8)=25.86, \mathrm{p}=0.001)$ and AML2 cells (Figure 5.2.13 A: Basal: $\mathrm{F}(2,8)=16.57, \mathrm{p}=0.004$; Figure 5.2.13 B: Maximal: $F(2,8)=36.75, p=0.001)$. Mitochondrial respiration at the $E T C$ is fuelled by multiple substrates including long chain fats by FAO (Martínez-Reyes \& Chandel, 2020). The rate of complete palmitate oxidation was assessed by quantifying the release of ${ }^{14} \mathrm{CO}_{2}$ after suppling leukemia cells with radiopalmitate (Huynh et al., 2014). AYNE inhibited FAO in TEX (Figure 5.2.13 C: TEX: $F(4,14)=27.28, p<0.001)$ and AML2 (Figure 5.2.13 D: $F(4,14)=48.59, p<0.001)$ in a dose dependent manner. Taken together, AYNE directly inhibited FAO and hindered mitochondrial respiration, which is partially fuelled by FAO. 
A

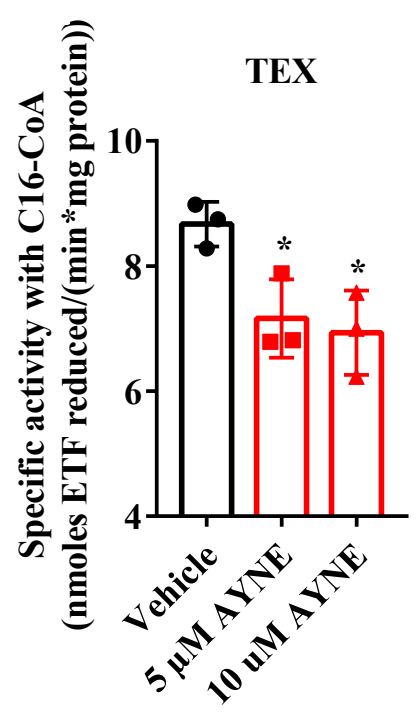

C

TEX

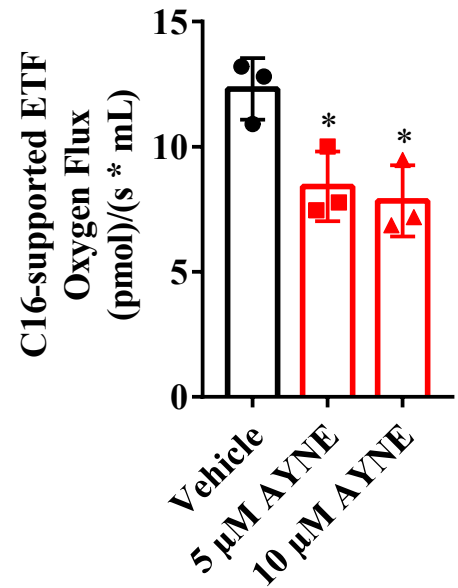

B

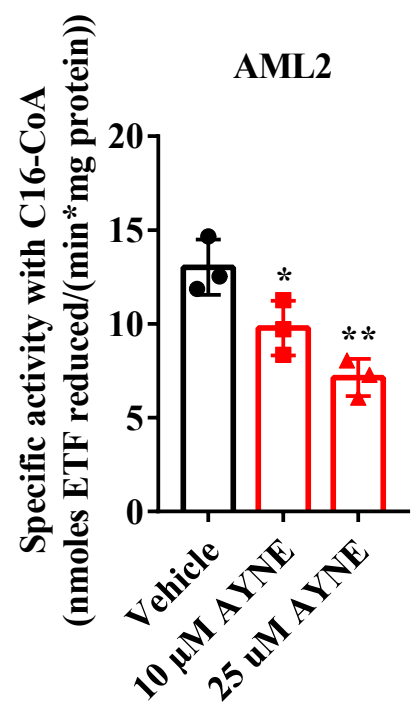

D

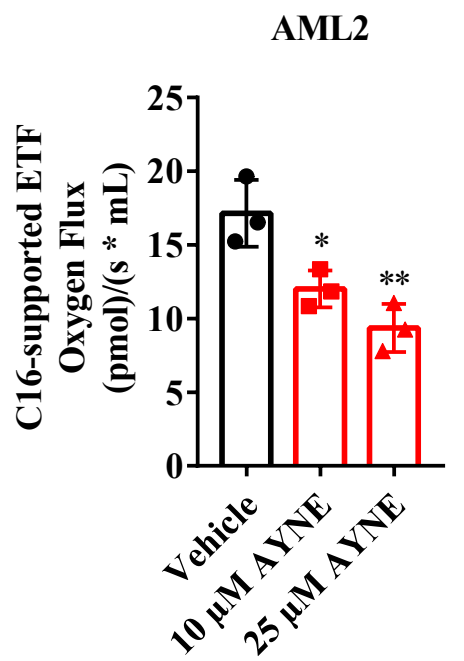

\section{Figure 5.2.14 AYNE inhibits mitochondrial FAO at VLCAD}

VLCAD activity following AYNE treatment was quantified by two assays. First, the loss of fluorescence upon ETF reduction is a direct quantification of VLCAD activity by a fluorescence spectrophotometer (Vockley et al., 2004). Following a 12-hour treatment with either a solvent vehicle or AYNE (at 10 or $25 \mu \mathrm{M}$ ), VLCAD activity in (A) TEX and (B) AML2 was directly quantified with a fluorescence-based assay with C16-CoA to assess VLCAD activity. Secondly, C16-supported ETF oxygen flux assessed by a high resolution respirometer is an indirect measure of VLCAD activity (Ojuka et al., 2016). Following a one-hour treatment with either a solvent vehicle or AYNE (at 10 or $25 \mu \mathrm{M}$ ), VLCAD activity in (C) TEX and (D) AML2 was indirectly quantified via ETF respiration with palmitoyl-carnitine (PC) to assess VLCAD activity. For (AD), data presented as mean \pm S.D., n.s. not significant; ${ }^{*} \mathrm{p} \leq 0.05 ; * * \mathrm{p} \leq 0.002 ; * * * \mathrm{p} \leq 0.001$, using a one-way ANOVA, Tukey's post-hoc test. Data in (A, B) generated by Prof. A. W. Mohsen and Prof. J. Vockley (University of Pittsburgh Medical Center). 


\subsubsection{AYNE Inhibits Mitochondrial FAO At VLCAD}

Using the direct assessment of VLCAD activity by fluorescence (Vockley et al., 2004), AYNE inhibited VLCAD in TEX (Figure 5.2.14 A: $F(2,8)=8.26, p=0.02$ ) and AML2 (Figure 5.2.14 B: $F(2,8)=14.79, p=0.005)$. The indirect assessment of VLCAD activity by respirometry (Ojuka et al., 2016) also confirmed VLCAD inhibition by AYNE in TEX (Figure 5.2.14 C: $\mathrm{F}(2,8)=9.741, \mathrm{p}=0.01)$ and AML2 (Figure 5.2.14 D: $\mathrm{F}(2,8)=14.97, \mathrm{p}=0.005)$. Taken together, two different methodologies assessing VLCAD activity confirmed VLCAD inhibition by AYNE in both TEX and AML2. 
A

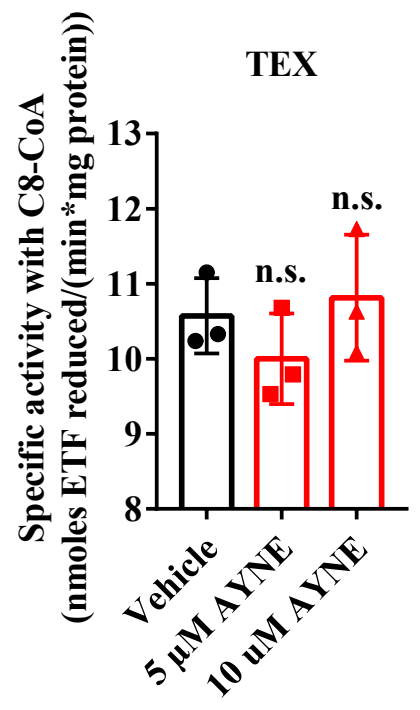

C

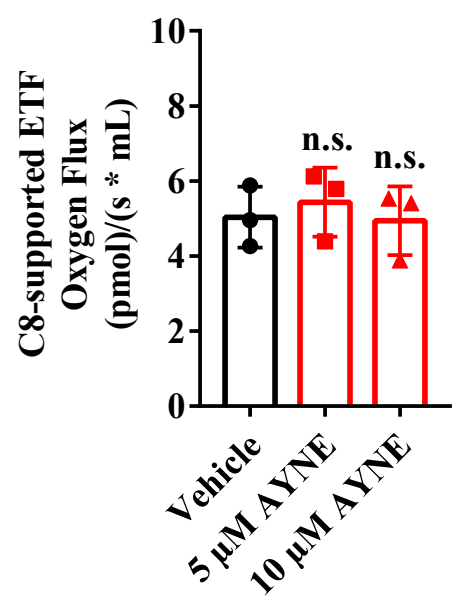

B

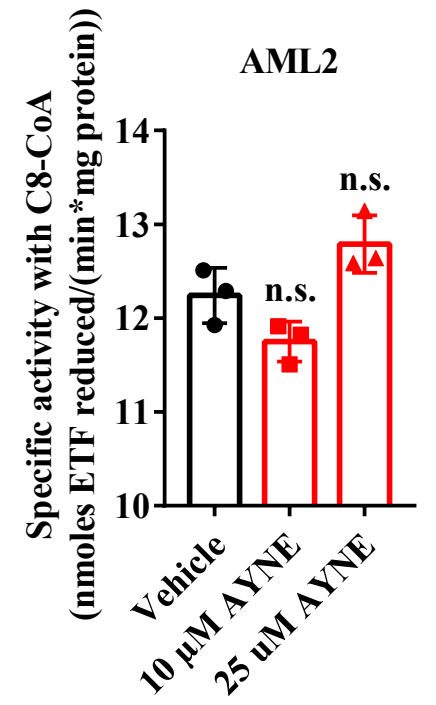

D

AML2
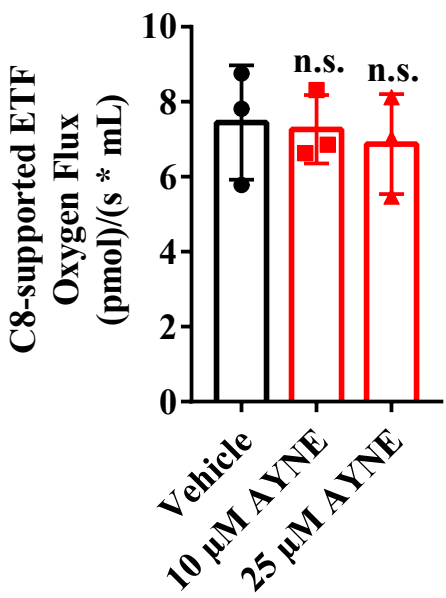

AML2

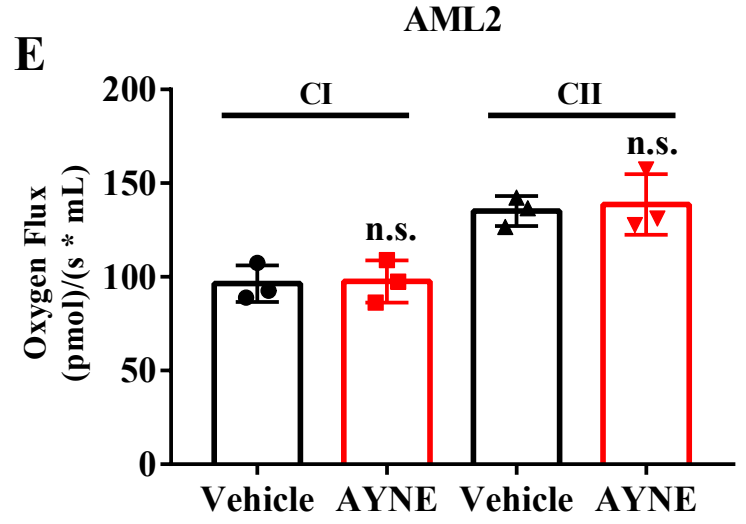


Figure 5.2.15 VLCAD inhibition by AYNE does not alter the activities of MCAD or complexes I and II of the ETC

MCAD activity following AYNE treatment was quantified by two assays (Vockley et al., 2004)(Ojuka et al., 2016). First, the loss of fluorescence upon ETF reduction is direct quantification of MCAD activity by a fluorescence spectrophotometer (Vockley et al., 2004). Following a 12-hour treatment with either a solvent vehicle or AYNE (at 10 or $25 \mu \mathrm{M}$ ), MCAD activity in (A) TEX and (B) AML2 was directly quantified with a fluorescence-based assay and octanoyl-CoA (C8-CoA). Secondly, C8-supported ETF oxygen flux assessed by a high resolution respirometer is an indirect measure of MCAD activity (Ojuka et al., 2016). Following a one-hour treatment with either a solvent vehicle or AYNE (at 10 or $25 \mu \mathrm{M}$ ), MCAD activity in (C) TEX and (D) AML2 was indirectly quantified via ETF respiration with octanoyl-carnitine (OC). (E) Following a one-hour treatment with either a solvent vehicle or $10 \mu \mathrm{M}$ AYNE, complex I and II respiration was assessed in AML using substrates specific to complex I (pyruvate, PYR; monosodium glutamate, MSG; malate, MAL) and II (succinate) that are not associated with the FAO pathway (Ojuka et al., 2016). For (A-D), data presented as mean \pm S.D., n.s. not significant; $* \mathrm{p} \leq 0.05 ; * * \mathrm{p} \leq 0.002 ; * * * \mathrm{p} \leq 0.001$, using a one-way ANOVA, Tukey's post-hoc test. For (E), data presented as mean \pm S.D., n.s. not significant, using a two-tailed, unpaired Student's $t$-test. Data in (A, B) generated by Prof. A. W. Mohsen and Prof. J. Vockley (University of Pittsburgh Medical Center). 


\subsubsection{VLCAD Inhibition By AYNE Does Not Alter The Activities Of MCAD Or Complexes I And II Of The ETC}

VLCAD and MCAD are both members of the acyl-CoA dehydrogenase family and both utilize glutamic acid as a catalytic residue to catalyze the unsaturation of long and medium chain fats, respectively (McAndrew et al., 2008). MCAD activity must be assessed to confirm that AYNE is a chemical inhibitor specific to VLCAD and not to the general ACAD enzyme family.

Using the direct assessment of MCAD activity by fluorescence, AYNE did not alter MCAD activity in TEX (Figure 5.2.15 A: $F(2,8)=1.193, p=0.37$ ) and AML2 (Figure 5.2.15 B: $\mathrm{F}(2,8)=10.8, \mathrm{p}=0.095)$. The indirect assessment of VLCAD activity by respirometry also confirmed VLCAD inhibition by AYNE in TEX (Figure 5.2.15 C: $F(2,8)=0.2599, p=0.78$ ) and AML2 (Figure 5.2.15 D: $F(2,8)=0.158, p=0.86)$.

The activities of complexes I and II in the ETC with non-FAO substrates were also assessed to further define AYNE's specificity to VLCAD and long chain FAO. In AML2, the respiration rates of complex I (Figure 5.2.15 E: t(4)=0.1366, p=0.9) and complex II (Figure 5.2.15 E: $\mathrm{t}(4)=0.3336, \mathrm{p}=0.76)$ were unaffected, confirming that these ETC complexes were not potential off-targets of AYNE at anti-AML doses. Taken together, AYNE specifically inhibited VLCAD activity, with no significant changes in the activities of MCAD as well as complexes I \& II of the ETC. 

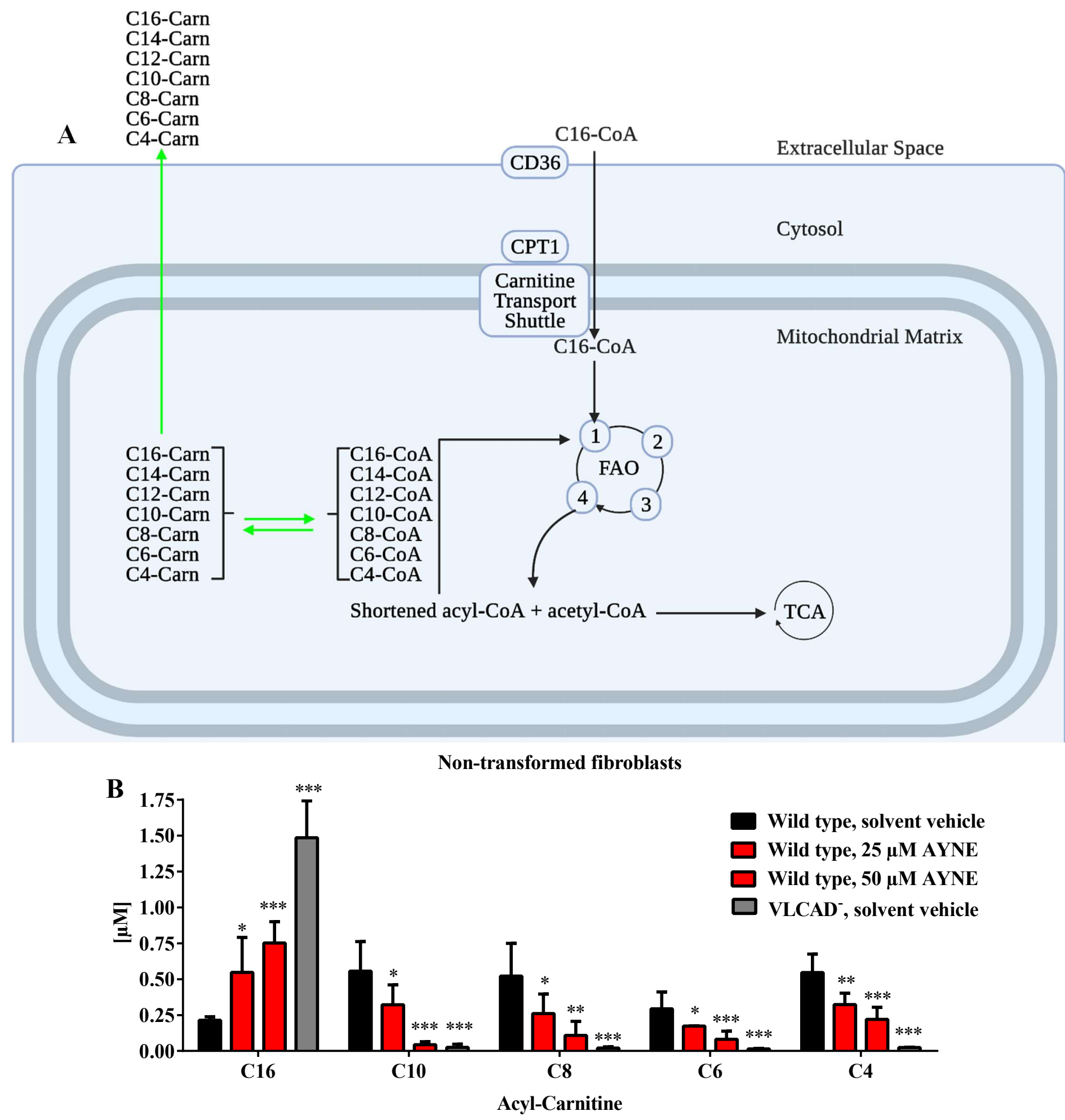

Figure 5.2.16 AYNE inhibits long chain mitochondrial FAO in non-transformed fibroblasts (A) Schematic of the mitochondrial FAO pathway highlighting the repeated shortening of long chain fats by two carbons. In the mitochondrial matrix, fatty acids coupled to the CoA cofactor are broken down to produce fatty acyl-CoA intermediates that are 16,14,12, ten, eight, six, four, or two carbons long. The acyl-CoA ester intermediates can be converted to and are in equilibrium with corresponding acyl-carnitine (AC) ester intermediates, through the action of mitochondrial acyl-CoA esterases (Equilibrium is highlighted by the two green arrows in the mitochondrial matrix). AC's of different lengths can be transported through the mitochondrial and cellular 
membranes to accumulate in extracellular space, allowing MS quantification (Release of AC's highlighted by green arrow starting in the matrix and ending in the extracellular space)(Okun et al., 2002). Abbreviations: CD36, cluster of differentiation 36; CPT1, carnitine palmitoyltransferase 1; FAO, fatty acid oxidation; TCA, tricarboxylic acid cycle; carn, L-carnitine cofactor; CoA, coenzyme A cofactor. Created in Biorender. (B) AC profiling of the spent media of human non-transformed fibroblasts (From either a healthy donor or VLCAD', a patient of VLCAD deficiency) treated with a solvent vehicle or AYNE (at 25 or $50 \mu \mathrm{M})$ for $96 \mathrm{hrs}(\mathrm{N}=4)$. For (B), data presented as mean \pm S.D., n.s. not significant; * $\mathrm{p} \leq 0.05 ; * * \mathrm{p} \leq 0.002 ; * * * \mathrm{p} \leq 0.001$, using a one-way ANOVA. Data in (B) generated by Prof. A. W. Mohsen and Prof. J. Vockley (University of Pittsburgh Medical Center). 


\subsubsection{AYNE Inhibits Long Chain Mitochondrial FAO In Non-Transformed Fibroblasts}

AC profiling is a clinical screening tool to diagnose patients with FAO deficiencies. In humans with functional FAO, fatty acyl-CoA's are rapidly processed by the FAO pathway, resulting in no accumulation of FAO intermediate metabolites. However, mutations in FAO enzymes affecting enzyme activity as well as pharmacological inhibition induce an abnormal accumulation of the inhibited enzyme's substrates. Therefore, inhibition at different FAO enzymes results in accumulations and depletions of specific FAO metabolites, depending on the enzyme's position along the FAO pathway. For example, C16-carnitine is the carnitine ester of C16-CoA, the primary VLCAD substrate, and is a critical marker of VLCAD inhibition. Due to the repeated cyclical nature of the FAO pathway, this blockage at long chain FAO also resulted in the depletion of downstream FAO intermediates, leading to a decrease in substrates of MCAD and SCAD. AcylCoA's can be converted to corresponding AC's through a trans-esterification reaction carried out by mitochondrial acyl-CoA esterases until an equilibrium between CoA and carnitine esters is reached, allowing export of carnitine esters to prevent an intramitochondrial accumulation of lipid metabolites (Figure 5.2.16 A). Unlike acyl-CoA's, AC's can be transported through the mitochondrial and cell membranes to accumulate in cell media, allowing MS quantification (Figure 5.2.16 A)(Okun et al., 2002).

To confirm long chain FAO inhibition at VLCAD, the AC profiles of non-transformed fibroblasts derived from a human donor with functional FAO were treated with a vehicle or AYNE; these profiles were compared to the AC profile of a patient of VLCAD deficiency (designated $\mathrm{VLCAD}^{-}$in (Figure 5.2.16 B)). AYNE treatment of the fibroblasts with functional FAO generated a similar AC profile to the AC profile of the VLCAD deficient patient with an characteristic accumulation of the fully-saturated C16-carnitine and characteristic depletions of medium and 
short chain fully saturated AC's, in line with FAO inhibition at VLCAD (Figure 5.2.16 B: C16carn: $\mathrm{F}(3,16)=52.78 ; \mathrm{p}<0.001 ; \mathrm{C} 10$-carn: $\mathrm{F}(3,16)=20.48 ; \mathrm{p}<0.001 ; \mathrm{C} 8$-carn: $\mathrm{F}(3,16)=12.10$ $\mathrm{p}<0.001$; C6-carn: $\mathrm{F}(3,16)=17.35 ; \mathrm{p}<0.001 ; \mathrm{C} 4$-carn: $\mathrm{F}(3,16)=31.83 ; \mathrm{p}<0.001)$. Taken together with the results of the liquid scintillation counting experiments (Figure 5.2.13) and assessment of VLCAD activity (Figure 5.2.14), these results further confirmed that AYNE inhibited the FAO pathway at VLCAD, across multiple cell types. 

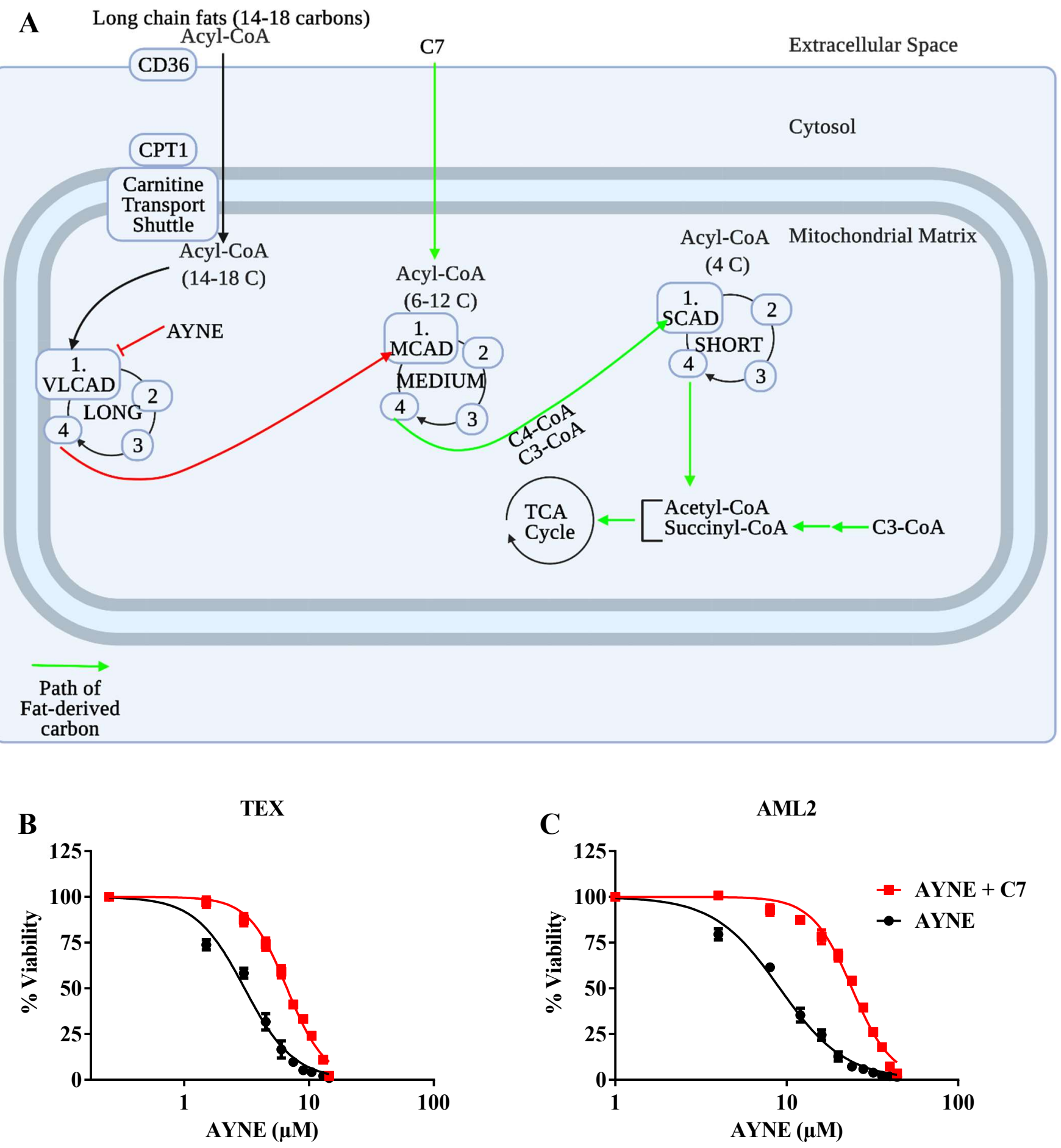

Figure 5.2.17 Supplementation with the medium chain fat sodium heptanoate rescues AML from long chain FAO inhibition by AYNE

(A) Schematic demonstrating sodium heptanoate (C7) partially attenuating AYNE-induced cell death. Upon AYNE treatment, long chain FAO was inhibited (red blunted arrow in schematic). Medium chain fats bypassed the long chain FAO enzymes to be oxidized by medium and short chain FAO enzymes, thus partially restoring FAO activity (green arrows in schematic) (Vockley et al., 2016). Abbreviations: C7, sodium heptanoate; CoA, coenzyme A; CPT1, carnitine 
palmitoyltransferase 1; CD36, cluster of differentiation 36; TCA, tricarboxylic acid cycle; VLCAD, very long chain acyl-CoA dehydrogenase; MCAD, medium chain acyl-CoA dehydrogenase; SCAD, short chain acyl-CoA dehydrogenase. Drawn in Biorender. Viability of (B) TEX and (C) AML2 cells following a $72 \mathrm{hr}$ AYNE treatment co-incubated with C7 (N=3). For $(\mathbf{B}, \mathbf{C}), \mathrm{IC}_{50}$ values were calculated using the dose response - inhibition equation on GraphPad Prism 7.0 and then compared as mean \pm S.D.; n.s. not significant; ${ }^{*} \mathrm{p} \leq 0.05 ; * * \mathrm{p} \leq 0.002 ; * * * \mathrm{p} \leq$ 0.001 , using a two-tailed, unpaired Student's $t$-test. 


\subsubsection{Supplementation With The Medium Chain Fat Sodium Heptanoate Rescues AML From Long Chain FAO Inhibition By AYNE}

Inhibition at a long chain FAO enzyme, such as VLCAD, prevents successive shortening of fat molecules, depriving acetyl-CoA bound for the TCA cycle and electrons bound for the ETC (red arrows: Figure 5.2.17 A). C7 is the salt of the active metabolite of triheptanoin, a glycerol esterified to three seven carbon long fatty acids, and is currently used as a dietary supplement to alleviate the symptoms of long chain FAO disorders. $\mathrm{C} 7$ bypasses the blockage at long chain FAO to be oxidized by medium and short chain FAO enzymes, partially restoring FAO activity (Green arrows: Figure 5.2.17 A). Propionyl-CoA produced from the oxidation of an odd number fat is converted to succinyl-CoA, a TCA cycle intermediate (Figure 5.2.17 A) (Vockley et al., 2016).

Co-treatment with $\mathrm{C} 7$ increased the $\mathrm{IC}_{50}$ value in $\mathrm{TEX}$ (Figure 5.2.17 B: $\mathrm{IC}_{50}$ with AYNE: $3.04 \pm 0.11 \mu \mathrm{M} ; \mathrm{IC}_{50}$ with AYNE $+\mathrm{C} 7: 6.67 \pm 0.32 \mu \mathrm{M}$ ) and AML2 (Figure 5.2.17 C: IC 50 with AYNE: $9.14 \pm 0.28 \mu \mathrm{M} ; \mathrm{IC}_{50}$ with AYNE $\left.+\mathrm{C} 7: 24.03 \pm 0.42 \mu \mathrm{M}\right) . \mathrm{C} 7$ significantly reduced AYNE-imparted cell toxicity in TEX (Figure 5.2.17 B: $t(4)=14.59, \mathrm{p}<0.001)$ and AML2 cells (Figure 5.2.17 C: $\mathfrak{t}(4)=12.25, \mathrm{p}<0.001)$. Taken together, medium chain fat supplementation attenuated AML death induced by inhibition of long chain FAO. 
AML\#19

A
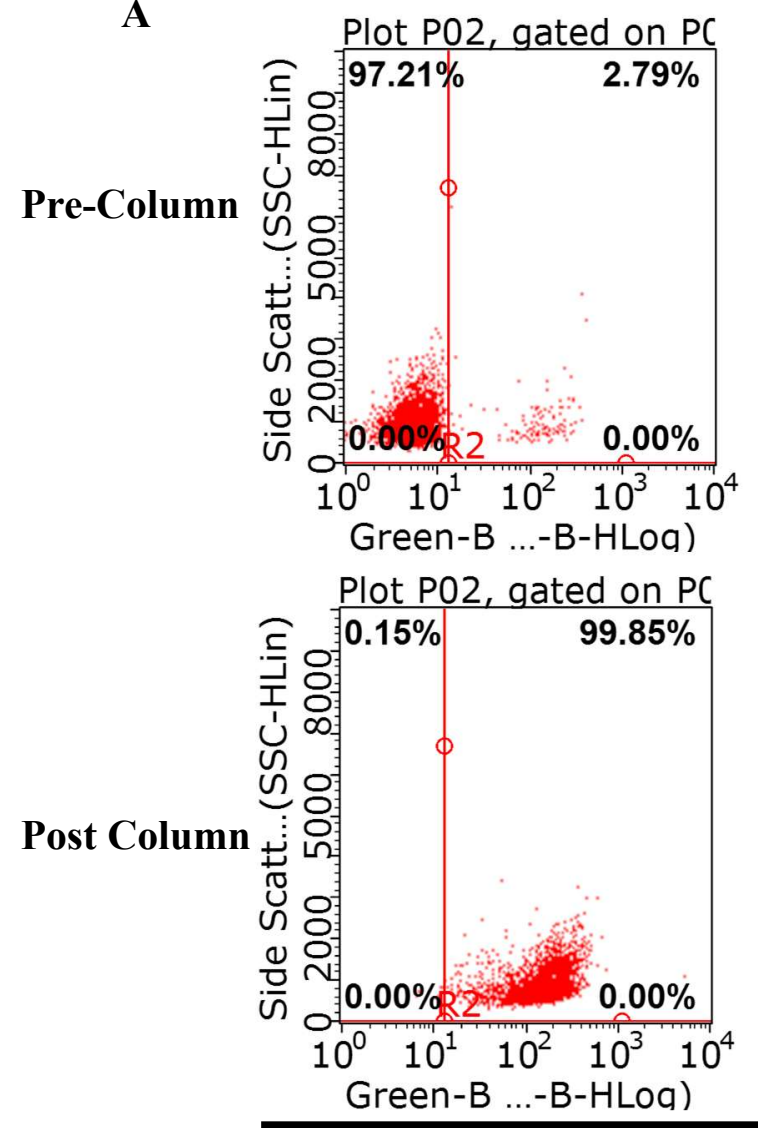

AML\#23
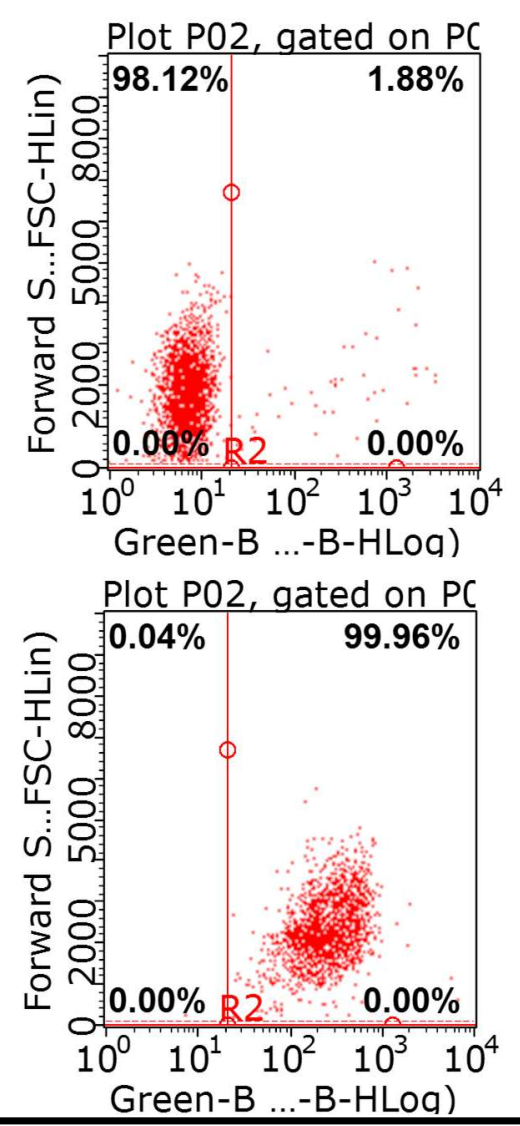

CD34
Normal MNC
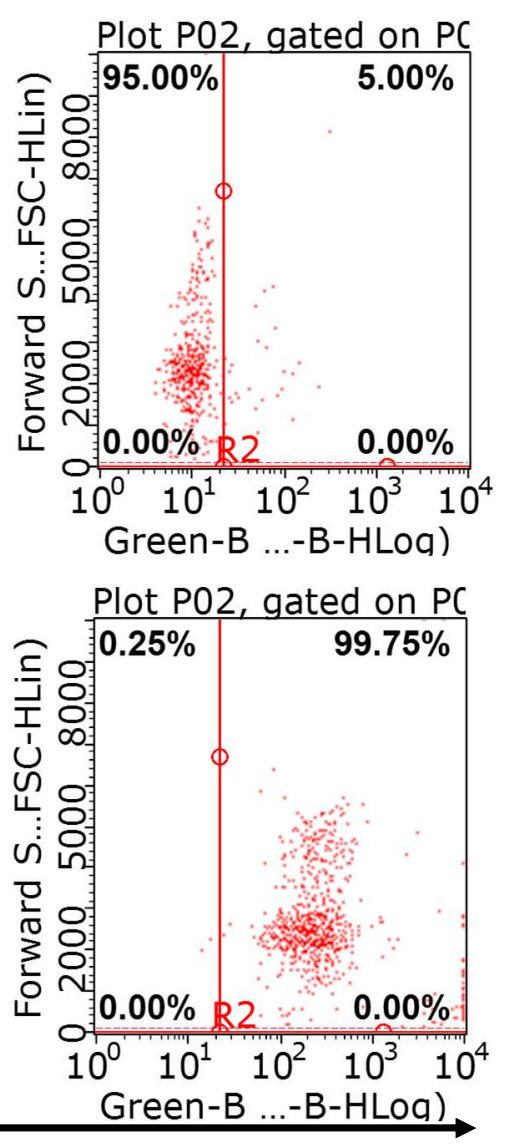

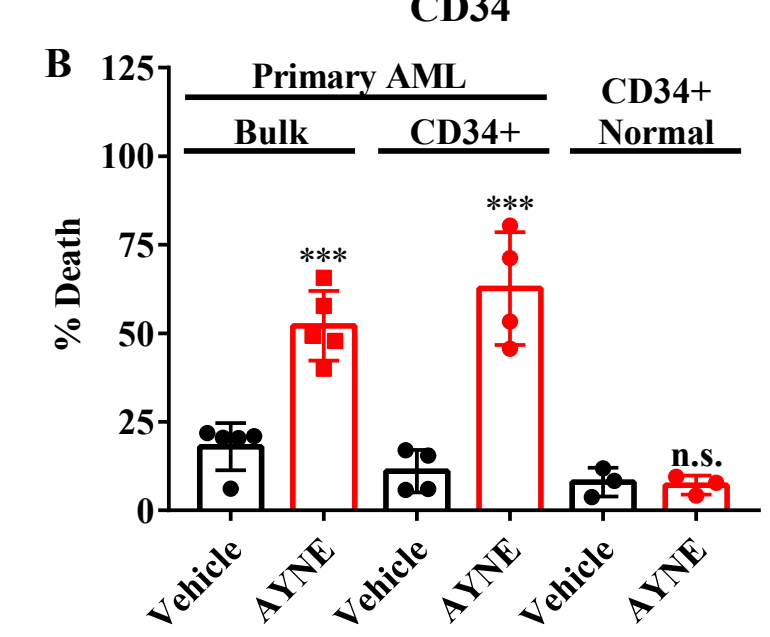

C

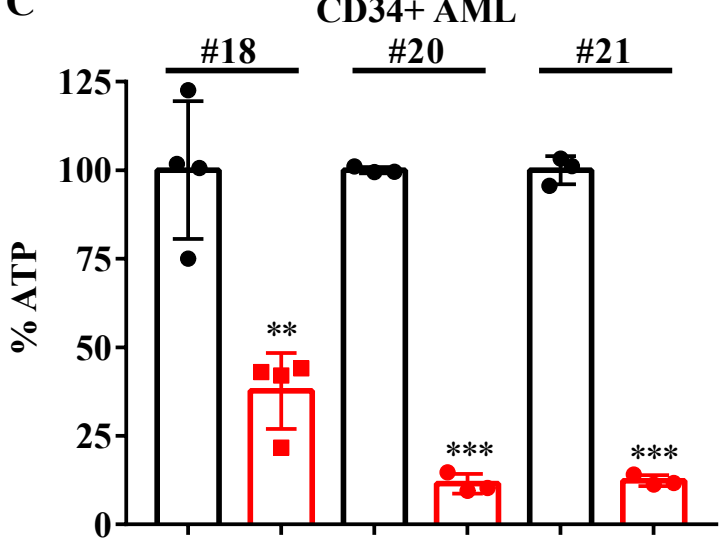

129
CD34+ Normal

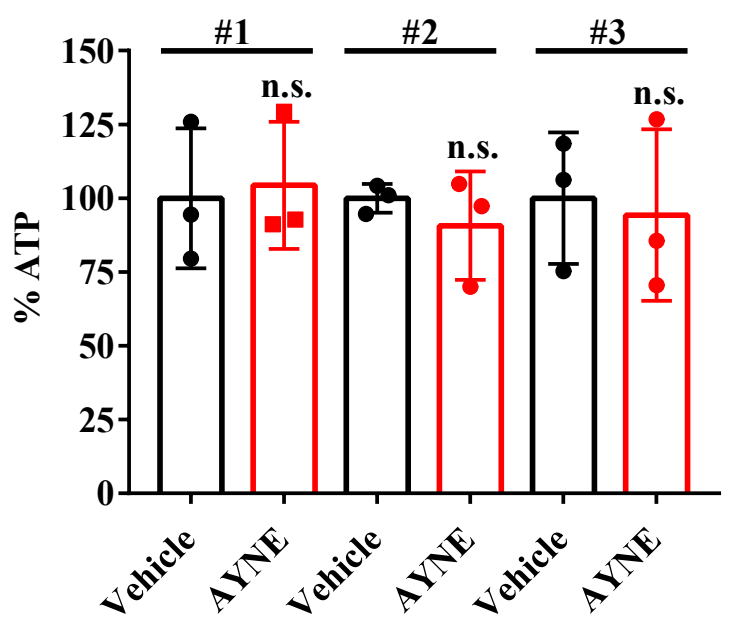


Figure 5.2.18 AYNE triggers ATP depletion to selectively induce death in CD34+ primary AML while sparing CD34+ normal hematopoietic cells

(A) Representative dot plots demonstrating column isolation of CD34+ subpopulations from primary AML and normal MNC samples. The isolated CD34+ subpopulations were then used for downstream assays (Figure 5.2.18 B-D). (B) Cell death of primary bulk AML (AML\#14 AML\#18), primary CD34+ AML (AML\#2, AML\#3, AML\#15, AML\#19), and normal CD34+ cells $(n=3)$ treated for $24 \mathrm{hrs}$. Quantification of ATP levels in (C) primary CD34+ AML and (D) CD34+ normal cells after a $12 \mathrm{hr}$ treatment. For $(\mathbf{B}, \mathbf{C}, \mathbf{D})$, primary CD34+ subpopulations were treated with either a solvent vehicle or $50 \mu \mathrm{M}$ AYNE. For $(\mathbf{B}, \mathbf{C}, \mathbf{D})$, data presented as mean \pm S.D., n.s. not significant; $* \mathrm{p} \leq 0.05 ; * * \mathrm{p} \leq 0.002 ; * * * \mathrm{p} \leq 0.001$, using a two-tailed, unpaired Student's $t$-test. See Table 5.2.14 for patient cytogenetics. 


\subsubsection{AYNE Triggers ATP Depletion And Selectively Induces Death In CD34+ Primary}

\section{AML While Sparing CD34+ Normal Hematopoietic Cells}

The cluster of differentiation 34 (CD34) is a glycosylated cell surface antigen that is highly expressed on primitive hematopoietic population but gradually lost when cells mature and differentiate (Reinisch et al., 2015). Compared to bulk hematopoietic cells, these CD34expressing, primitive subpopulations exhibit increased self-renewal, the capacity to engraft, and are metabolically distinct, compared to the bulk population (Holyoake \& Alcorn, 1994).

The AML and normal CD34+ subpopulations were isolated from the corresponding bulk populations (Figure 5.2.18 A), prior to treatment with AYNE. VLCAD inhibition by AYNE did not alter CD34+ normal cell viability (Figure 5.2.18 B: $\mathrm{t}(4)=0.2992, \mathrm{p}=0.78$ ) but induced cell death of bulk primary AML (Figure 5.2.18 B: $\mathrm{t}(4)=6.793, \mathrm{p}=0.002)$ and CD34+ primary AML cells (Figure 5.2.18 B: t(6)=6.047, $\mathrm{p}<0.001)$. Preceding AML cell death, ATP levels in CD34+ AML were depleted (Figure 5.2.18 C: AML\#18: $\mathrm{t}(6)=5.609, \mathrm{p}=0.001 ; A M L \# 20: \mathrm{t}(4)=52.23$, $\mathrm{p}<0.001$; AML\#21: $\mathrm{t}(4)=35.41, \mathrm{p}<0.001)$ while normal CD34+ were spared (Figure 5.2.18 D: Normal CD34+ \#1: $\mathrm{t}(4)=0.239, \mathrm{p}=0.82 ; \# 2: \mathrm{t}(4)=0.8484, \mathrm{p}=0.44 ; \# 3: \mathrm{t}(4)=0.2689, \mathrm{p}=0.8)$. Taken together, AYNE triggered an energy crisis preceding AML death. At the same dose and timepoint, normal cells were not affected. 


\section{A}

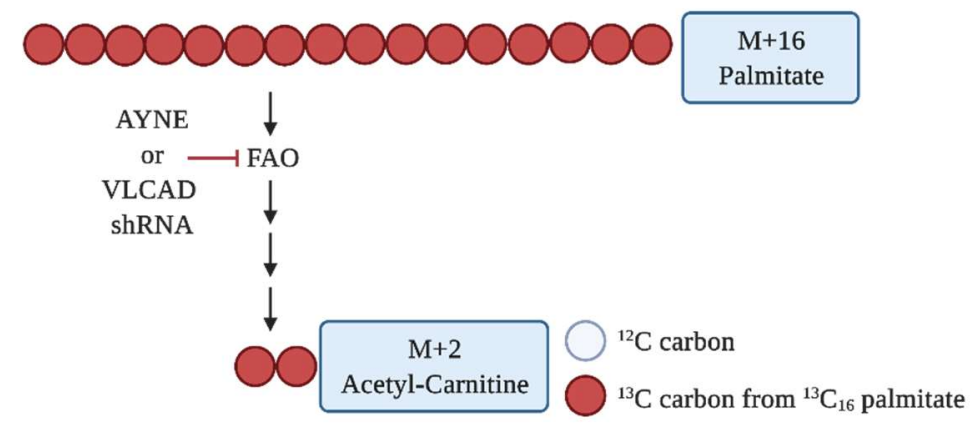

B

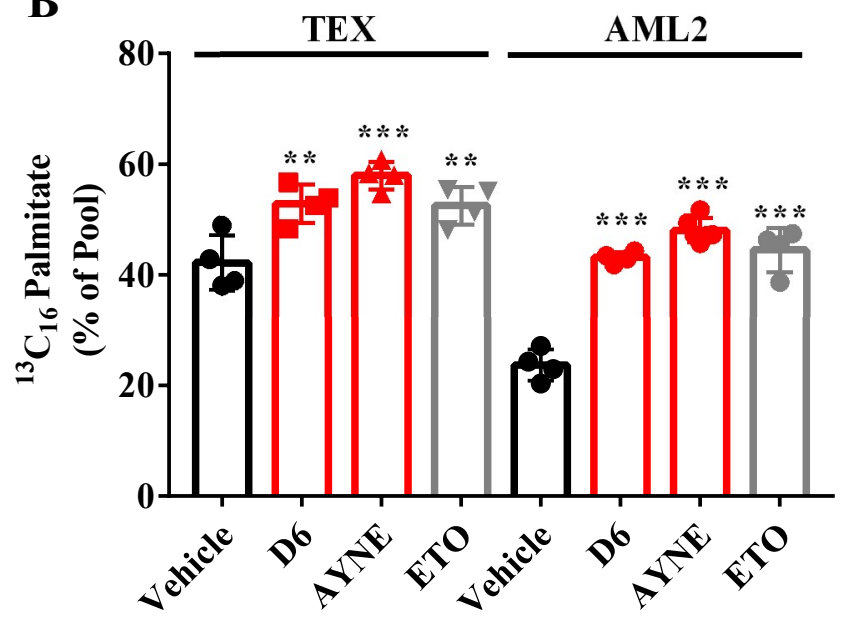

C

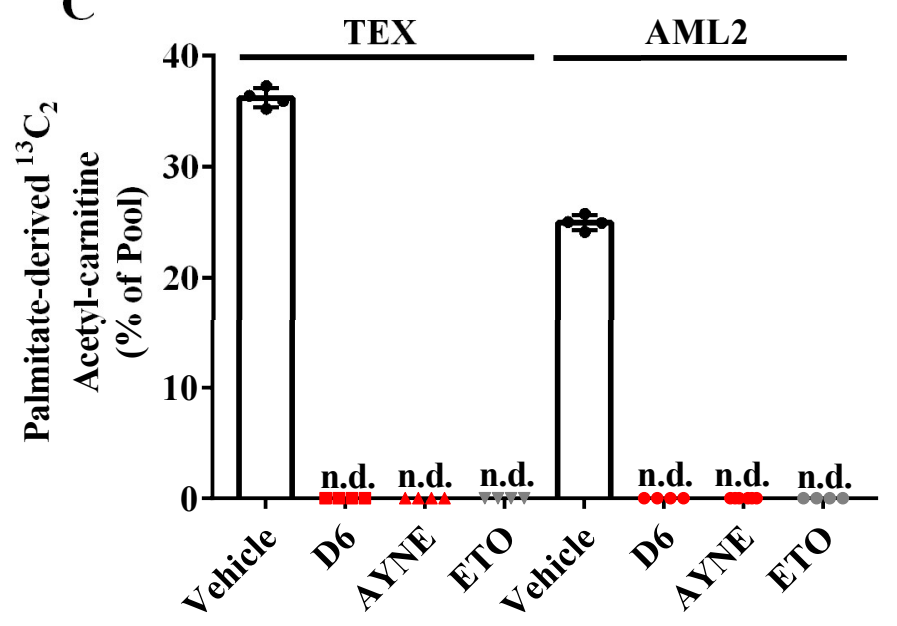

Figure 5.2.19 AYNE inhibits FAO activity in TEX and AML2

(A) Schematic outlining stable isotope experiments where ${ }^{13} \mathrm{C}_{16}$ palmitate (also $\mathrm{M}+16$ palmitate) was supplied to AML cell lines (Jones et al., 2018). The levels of M+16 palmitate, the initial substrate for the FAO pathway, and ${ }^{13} \mathrm{C}_{2}$ acetyl-carnitine (also $\mathrm{M}+2$ acetyl-carnitine), the final product of the FAO, were assessed in TEX and AML2 after a $12 \mathrm{hr}$ AYNE treatment. Red circles represent ${ }^{13} \mathrm{C}$ isotopic atoms derived from the ${ }^{13} \mathrm{C}_{16}$ palmitate tracer and grey circles represent ${ }^{12} \mathrm{C}$ atoms. Abbreviations: ${ }^{13} \mathrm{C}$, carbon 13 isotope; AYNE, avocadyne; VLCAD, very long chain acylCoA dehydrogenase; shRNA, short hairpin RNA; FAO, fatty acid oxidation. Created in Biorender. Levels of (B) ${ }^{13} \mathrm{C}_{16}$ palmitate and (C) ${ }^{13} \mathrm{C}_{2}$ acetyl-carnitine in TEX and AML2 following treatment with either DMSO (for vehicle and D6 cells), AYNE (10 $\mu \mathrm{M}$ for TEX and $25 \mu \mathrm{M}$ for AML2), or $100 \mu \mathrm{M}$ ETO. For (B, C), data presented as mean \pm S.D., n.s. not significant; * $\mathrm{p} \leq 0.05 ;{ }^{* *} \mathrm{p} \leq 0.002$; $* * * p \leq 0.001$, using a one-way ANOVA, Tukey's post-hoc test. For $(\mathbf{B}, \mathbf{C})$, metabolites that were below the limit of detection are labelled as not detected (n.d.). The statistical summaries are tabulated below in Table 5.2.9. MS analysis carried by E. Bohrnsen and Prof. A. D'Alessandro (University of Colorado Anschutz Medical Campus). 


\subsubsection{AYNE Inhibits FAO Activity In TEX And AML2}

The levels of ${ }^{13} \mathrm{C}_{16}$ palmitate, the starting material of the FAO pathway and primary substrate of the VLCAD enzyme, as well as ${ }^{13} \mathrm{C}_{2}$ acetyl-carnitine, the final product of the FAO pathway, in TEX and AML2, following VLCAD knockdown or treatment with either AYNE or ETO, were quantified to assess FAO activity (Figure 5.2.19 A).

In TEX and AML2, shRNA knockdown of VLCAD as well as treatment with AYNE and ETO, a positive control for FAO inhibition (Samudio et al., 2010), increased intracellular ${ }^{13} \mathrm{C}_{16}$ palmitate (Figure 5.2.19 B) and decreased ${ }^{13} \mathrm{C}_{2}$ acetyl-carnitine (below the detection limit) confirmed long chain FAO inhibition (Figure 5.2.19 C). Taken together, stable isotope tracing experiments with ${ }^{13} \mathrm{C}_{16}$ palmitate confirmed FAO inhibition following genetic ablation of VLCAD or treatment with an FAO inhibitor in TEX and AML2. The statistical summaries are tabulated below in Table 5.2.9. 


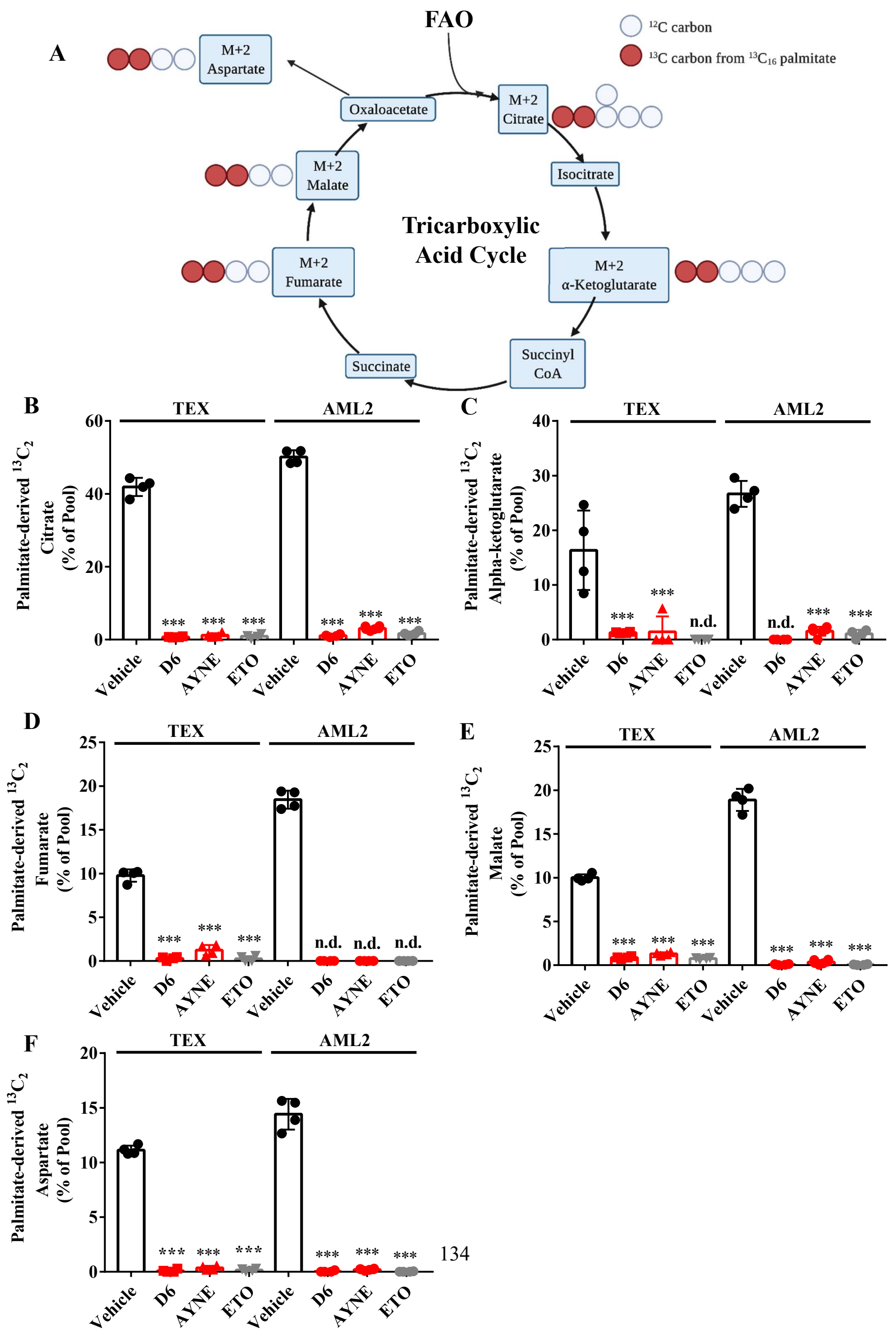


Figure 5.2.20 AYNE inhibits FAO contribution into the TCA cycle in TEX and AML2

(A) Schematic showing enrichment of fat-derived carbon $13\left({ }^{13} \mathrm{C}\right)$ atoms from palmitate-derived ${ }^{13} \mathrm{C}_{2}$ (also $\mathrm{M}+2$ ) acetyl-carnitine into ${ }^{13} \mathrm{C}_{2}$ TCA metabolites (Jones et al., 2018) following a $12 \mathrm{hr}$ treatment in TEX and AML2 cell lines. Red circles represent ${ }^{13} \mathrm{C}$ isotopic atoms derived from the ${ }^{13} \mathrm{C}_{16}$ palmitate tracer and grey circles represent ${ }^{12} \mathrm{C}$ atoms. Abbreviations: FAO, fatty acid oxidation; CoA, coenzyme A. Created in Biorender. Levels of (B) ${ }^{13} \mathrm{C}_{2}$ citrate, (C) ${ }^{13} \mathrm{C}_{2} \alpha$ ketoglutarate, (D) ${ }^{13} \mathrm{C}_{2}$ fumarate, (E) ${ }^{13} \mathrm{C}_{2}$ malate, (F) ${ }^{13} \mathrm{C}_{2}$ aspartate in TEX and AML2 following treatment with either DMSO (for vehicle and D6 cells), AYNE (10 $\mu \mathrm{M}$ for TEX and $25 \mu \mathrm{M}$ for AML2), or $100 \mu \mathrm{M}$ ETO. For (A, B), data presented as mean \pm S.D., n.s. not significant; * $\mathrm{p} \leq 0.05$; ** $\mathrm{p} \leq 0.002$; *** $\mathrm{p} \leq 0.001$, using a one-way ANOVA, Tukey's post-hoc test. For (B-F), metabolites that were below the limit of detection are labelled as not detected (n.d.). Statistical summaries are tabulated below in Table 5.2.9. MS analysis carried by E. Bohrnsen and Prof. A. D’Alessandro (University of Colorado Anschutz Medical Campus). 
Table 5.2.9 Summary of statistics for data presented in figure 5.2.19 and 5.2.20.

\begin{tabular}{|c|c|c|c|c|c|c|}
\hline \multirow[b]{3}{*}{ Isotopomer } & \multicolumn{6}{|c|}{ Cell Line } \\
\hline & \multicolumn{3}{|c|}{ TEX } & \multicolumn{3}{|c|}{ AML2 } \\
\hline & F-value & P-value & Exclusions & F-value & P-value & Exclusions \\
\hline${ }^{13} \mathrm{C}_{16}$ Palmitate & $F(3,15)=12.88$ & $<0.001$ & None & $F(3,17)=76.07$ & $<0.001$ & None \\
\hline $\begin{array}{c}{ }^{13} \mathrm{C}_{2} \text { Acetyl- } \\
\text { Carnitine }\end{array}$ & N/A & N/A & $\begin{array}{c}\text { All } \\
\text { treatments } \\
\text { decreased } \\
\text { this } \\
\text { metabolite } \\
\text { below the } \\
\text { limit of } \\
\text { detection } \\
\text { (L.O.D.) }\end{array}$ & N/A & N/A & $\begin{array}{c}\text { All } \\
\text { treatments } \\
\text { decreased } \\
\text { this } \\
\text { metabolite } \\
\text { below the } \\
\text { L.O.D. }\end{array}$ \\
\hline${ }^{13} \mathrm{C}_{2}$ Citrate & $\mathrm{F}(3,15)=986.3$ & $<0.001$ & None & $\mathrm{F}(3,17)=2619$ & $<0.001$ & None \\
\hline $\begin{array}{c}{ }^{13} \mathrm{C}_{2} \text { Alpha- } \\
\text { ketoglutarate }\end{array}$ & $F(2,11)=14.77$ & 0.001 & $\begin{array}{l}\text { Vehicle vs. } \\
\text { ETO } \\
\text { excluded } \\
\text { as ETO } \\
\text { decreased } \\
\text { this } \\
\text { metabolite } \\
\text { below the } \\
\text { L.O.D. } \\
\end{array}$ & $F(2,13)=458.8$ & $<0.001$ & $\begin{array}{c}\text { Vehicle vs. } \\
\text { D6 } \\
\text { excluded as } \\
\text { D6 } \\
\text { decreased } \\
\text { this } \\
\text { metabolite } \\
\text { below the } \\
\text { L.O.D. }\end{array}$ \\
\hline${ }^{13} \mathrm{C}_{2}$ Fumarate & $F(3,15)=351.6$ & $<0.001$ & None & N/A & N/A & $\begin{array}{c}\text { All } \\
\text { treatments } \\
\text { decreased } \\
\text { this } \\
\text { metabolite } \\
\text { below the } \\
\text { limit of } \\
\text { detection. }\end{array}$ \\
\hline${ }^{13} \mathrm{C}_{2}$ Malate & $F(3,15)=1625$ & $<0.001$ & None & $F(3,17)=1007$ & $<0.001$ & None \\
\hline${ }^{13} C_{2}$ Aspartate & $F(3,15)=2082$ & $<0.001$ & None & $F(3,17)=495$ & $<0.001$ & None \\
\hline
\end{tabular}




\subsubsection{AYNE Inhibits FAO Contribution Into The TCA Cycle In TEX And AML2}

A major fate of fat-derived ${ }^{13} \mathrm{C}_{2}$ (also $\left.\mathrm{M}+2\right)$ acetyl-carnitine is entry into the TCA cycle and incorporation into the ${ }^{13} \mathrm{C}_{2}$ TCA metabolite pool (Jones et al., 2018). Levels of ${ }^{13} \mathrm{C}_{2}$ TCA metabolites were assessed in TEX and AML2 following genetic ablation of VLCAD or treatment with FAO inhibitors AYNE and ETO (Figure 5.2.20 A). Entry of fat derived carbon was significantly depleted in TEX and AML2, following FAO inhibition by either pharmacological blockade or genetic ablation of VLCAD (Figure 5.2.20 B-F). These results confirmed that FAO inhibition suppressed fat contribution to the TCA cycle in AML cells. 
A
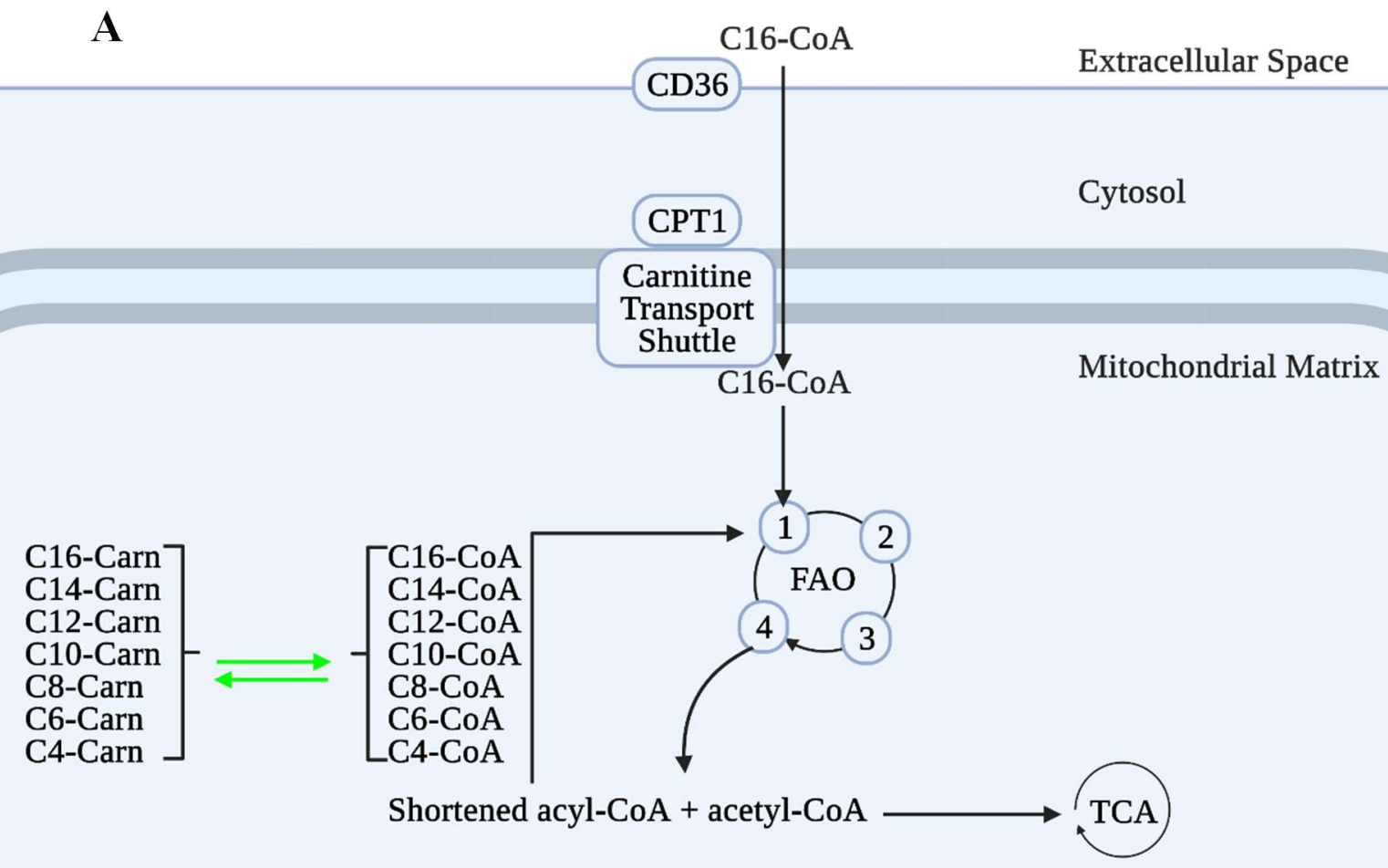

B

Primary AML
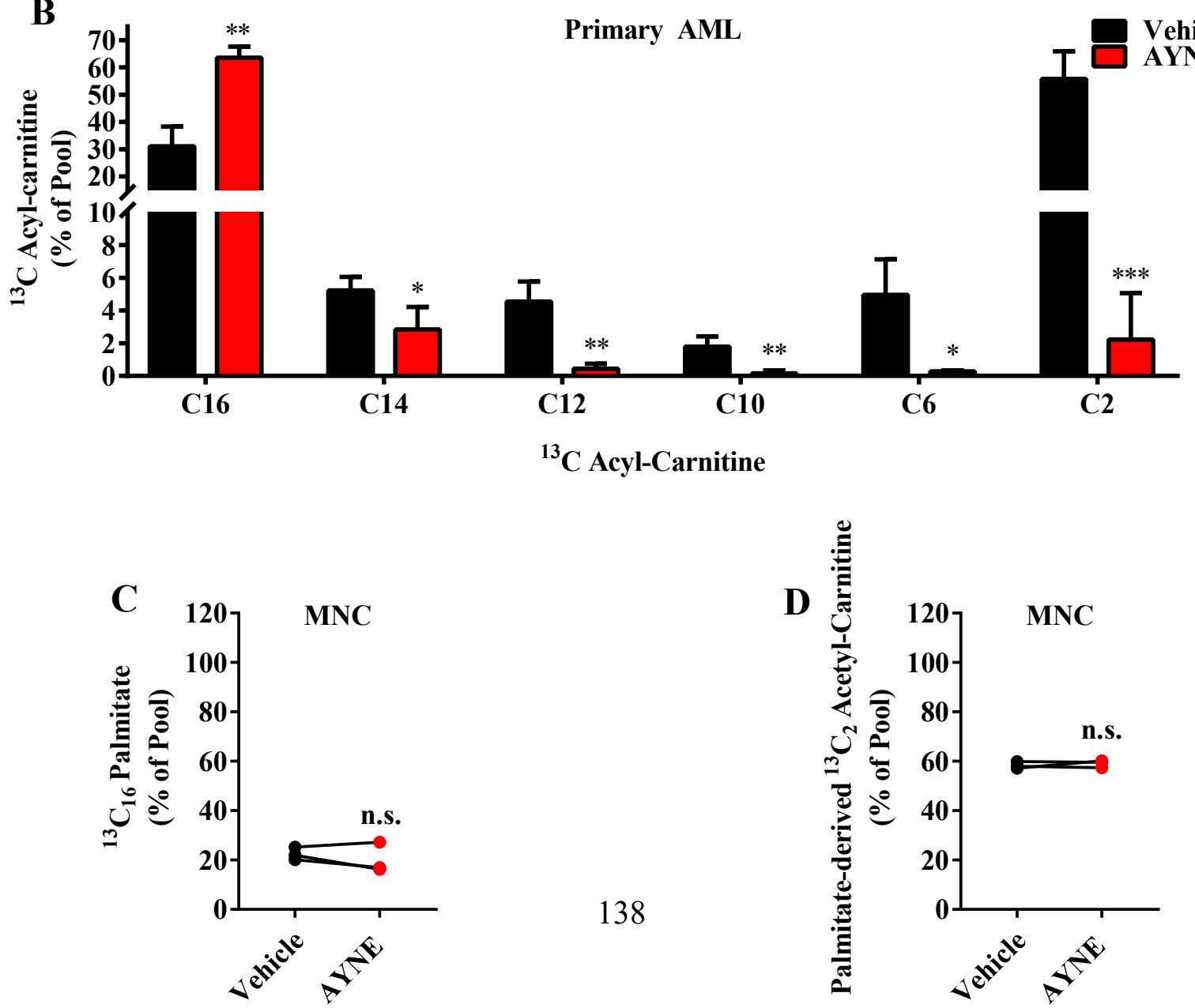
Figure 5.2.21 AYNE exclusively inhibits FAO in primary AML, while sparing normal MNC (A) Schematic of the mitochondrial FAO pathway highlighting the intramitochondrial equilibrium between acyl-CoA and AC intermediates highlighted in green arrows (Okun et al., 2002). Abbreviations: CoA, coenzyme A cofactor; C16-CoA, palmitoyl-CoA; CD36, cluster of differentiation 36; CPT1, carnitine palmitoyltransferase 1; carn, L-carnitine cofactor; FAO, fatty acid oxidation; TCA, tricarboxylic acid cycle. Created in Biorender. (B) Levels of ${ }^{13} \mathrm{C}$ acylcarnitine derived from ${ }^{13} \mathrm{C}_{16}$ palmitate in primary AML (AML\#4, AML\#22, AML\#23, AML\#24, AML\#27). Levels of (C) ${ }^{13} \mathrm{C}_{16}$ palmitate and (D) ${ }^{13} \mathrm{C}_{2}$ (also $\mathrm{M}+2$ ) acetyl-carnitine in normal MNC. For $(\mathbf{B}, \mathbf{C}, \mathbf{D})$, cells were treated for $12 \mathrm{hr}$ treatment with a vehicle control or $50 \mu \mathrm{M}$ AYNE. For (B, C, D), data presented as mean \pm S.D., n.s. not significant; * $\mathrm{p} \leq 0.05 ; * * \mathrm{p} \leq 0.002 ; * * * \mathrm{p} \leq 0.001$, using a two-tailed, unpaired Student's $t$-test. Summary of patient cytogenetics are tabulated in Table 5.2.14. Summary of statistics are tabulated below in Table 4.2.10 and Table 4.2.11. MS analysis carried by E. Bohrnsen and Prof. A. D'Alessandro (University of Colorado Anschutz Medical Campus). 


\subsubsection{AYNE Exclusively Inhibits FAO In Primary AML, While Sparing Normal MNC}

Having demonstrated FAO inhibition in TEX and AML2 using a ${ }^{13} \mathrm{C}_{16}$ palmitate tracer (Figure 5.2.19), FAO activity using a stable isotope palmitate tracer was assessed in primary AML and normal MNC cells following AYNE treatment. In contrast to figure 5.2.16, this experiment treated cells with AYNE for 12 hours and extracted the lipid content of cells for analysis; this is in contrast to the experiment presented in figure 5.2.21 A which treated cells for 96 hours and extracted the lipid content of the spent media for MS analysis.

In primary AML, FAO inhibition at VLCAD was confirmed due to the accumulation of ${ }^{13} \mathrm{C}_{16}$-carnitine, the starting material of the FAO pathway and the primary substrate of VLCAD, as well as the depletion of ${ }^{13} \mathrm{C}_{2}$ acetyl-carnitine, the final product of the FAO pathway (Figure 5.2.21 B). Significant depletion of acyl-carnitines 14,12 , ten, and six carbons in length confirmed that a blockade of long chain FAO slowed flux through the entire FAO pathway (Figure 5.2.21 B). In contrast, at the same dose and timepoint that inhibited FAO in AML, FAO activity in normal MNC samples were unaffected, demonstrating no change in ${ }^{13} \mathrm{C}_{16}$ palmitate (Figure 5.2.21 C) and ${ }^{13} \mathrm{C}_{2}$ acetyl-carnitine (Figure 5.2.21 D). 

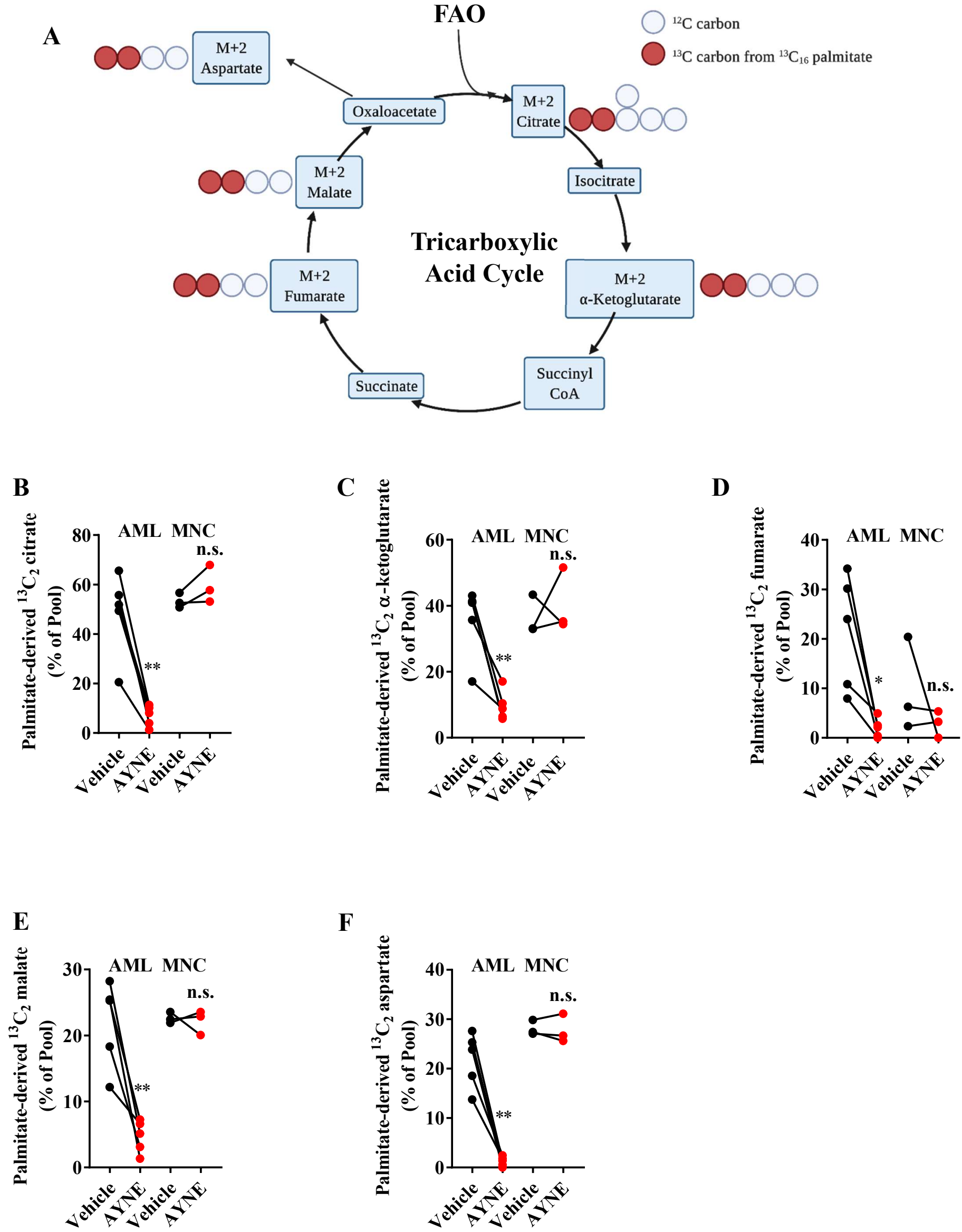
Figure 5.2.22 AYNE exclusively inhibits fat contribution into the TCA cycle in primary AML, while sparing normal MNC

(A) Schematic showing enrichment of fat-derived carbon $13\left({ }^{13} \mathrm{C}\right)$ atoms from palmitate-derived ${ }^{13} \mathrm{C}_{2}$ (also $\mathrm{M}+2$ ) acetyl-carnitine into ${ }^{13} \mathrm{C}_{2}$ tricarboxylic acid (TCA) metabolites following a $12 \mathrm{hr}$ AYNE treatment in primary AML and normal MNC cells. Red circles represent ${ }^{13} \mathrm{C}$ isotopes derived from the ${ }^{13} \mathrm{C}_{16}$ palmitate tracer and grey circles represent ${ }^{12} \mathrm{C}$ atoms. Abbreviations: FAO, fatty acid oxidation; CoA, coenzyme A. Created in Biorender. Levels of (B) ${ }^{13} \mathrm{C}_{2}$ citrate, (C) ${ }^{13} \mathrm{C}_{2}$ $\alpha$-ketoglutarate, (D) ${ }^{13} \mathrm{C}_{2}$ fumarate, (E) ${ }^{13} \mathrm{C}_{2}$ malate, (F) ${ }^{13} \mathrm{C}_{2}$ aspartate in primary AML and normal MNC cells treated with either a DMSO vehicle control or $50 \mu \mathrm{M}$ AYNE. For (B-F), data presented as mean \pm S.D., n.s. not significant; $* \mathrm{p} \leq 0.05$; ** $\mathrm{p} \leq 0.002 ; * * * \mathrm{p} \leq 0.001$, using a two-tailed, unpaired Student's $t$-test. Summary of patient cytogenetics are tabulated in Table 5.2.14. Summary of statistics are tabulated below in Table 5.2.10 and Table 5.2.11. MS analysis carried by E. Bohrnsen and Prof. A. D’Alessandro (University of Colorado Anschutz Medical Campus).

Table 5.2.10 Summary of statistics for data presented in Figure 5.2.21

\begin{tabular}{|c|c|c|}
\hline \multirow{2}{*}{ Isotopomer } & \multicolumn{2}{|c|}{ Primary AML } \\
\cline { 2 - 3 } & $\mathrm{t}(\mathrm{df})$ & P-value \\
\hline${ }^{13} \mathbf{C}_{\mathbf{1 6}}$ & $5.639(4)$ & 0.005 \\
\hline${ }^{13} \mathbf{C}_{\mathbf{1 4}}$ & $3.49(3)$ & 0.04 \\
\hline${ }^{13} \mathbf{C}_{\mathbf{1 2}}$ & $7.273(3)$ & 0.005 \\
\hline${ }^{13} \mathbf{C}_{\mathbf{1 0}}$ & $7.249(3)$ & 0.005 \\
\hline${ }^{13} \mathbf{C}_{\mathbf{6}}$ & $4.297(3)$ & 0.02 \\
\hline${ }^{13} \mathbf{C}_{\mathbf{2}}$ & $4.849(4)$ & 0.008 \\
\hline
\end{tabular}

Table 5.2.11 Summary of statistics for data presented in Figure 5.2.21 and 5.2.22

\begin{tabular}{|c|c|c|c|c|}
\hline \multirow[b]{3}{*}{ Isotopomer } & \multicolumn{4}{|c|}{ Cell Type } \\
\hline & \multicolumn{2}{|c|}{ Primary AML } & \multicolumn{2}{|c|}{ Normal MNC } \\
\hline & $\mathrm{t}(4)$ & P-value & $\mathrm{t}(2)$ & $\mathrm{P}$-value \\
\hline${ }^{13} \mathrm{C}_{16}$ Palmitate & $\begin{array}{l}\text { Table } \\
4.2 .11\end{array}$ & $\begin{array}{l}\text { Table } \\
4.2 .11\end{array}$ & 1.012 & 0.42 \\
\hline $\begin{array}{c}{ }^{13} \mathrm{C}_{2} \text { Acetyl- } \\
\text { Carnitine }\end{array}$ & $\begin{array}{l}\text { Table } \\
4.2 .11\end{array}$ & $\begin{array}{l}\text { Table } \\
4.2 .11\end{array}$ & 0.5722 & 0.62 \\
\hline${ }^{13} C_{2}$ Citrate & 6.761 & 0.002 & 1.994 & 0.18 \\
\hline $\begin{array}{l}{ }^{13} \mathrm{C}_{2} \text { Alpha- } \\
\text { ketoglutarate }\end{array}$ & 4.828 & 0.008 & 0.4958 & 0.67 \\
\hline${ }^{13} C_{2}$ Fumarate & 3.674 & 0.02 & 0.9978 & 0.42 \\
\hline${ }^{13} C_{2}$ Malate & 5.157 & 0.007 & 0.2978 & 0.79 \\
\hline${ }^{13} C_{2}$ Aspartate & 7.384 & 0.002 & 0.3369 & 0.77 \\
\hline
\end{tabular}




\subsubsection{AYNE Exclusively Inhibits Fat Contribution Into The TCA Cycle In Primary}

\section{AML, While Sparing Normal MNC}

Having demonstrated that FAO inhibition decreased fat contribution into the TCA cycle in AML cell lines (Figure 5.2.20), the levels of ${ }^{13} \mathrm{C}_{2}$ (also $\left.\mathrm{M}+2\right)$ TCA metabolites were quantified to assess TCA activity in primary AML and normal MNC samples treated with AYNE (Figure 5.2.22 A). FAO inhibition by AYNE suppressed entry of fat-derived carbon into the TCA cycle exclusively in primary AML, while the levels of ${ }^{13} \mathrm{C}_{2}$ TCA metabolites in normal MNC were unaffected (Figure 5.2.22 B-F). Taken together, the results in Figures 5.2.21 and 5.2.22 confirmed that, at the same dose and timepoint, AYNE inhibited FAO activity and suppressed entry of fatderived carbon into the TCA cycle in primary AML, while sparing normal MNC cells. 


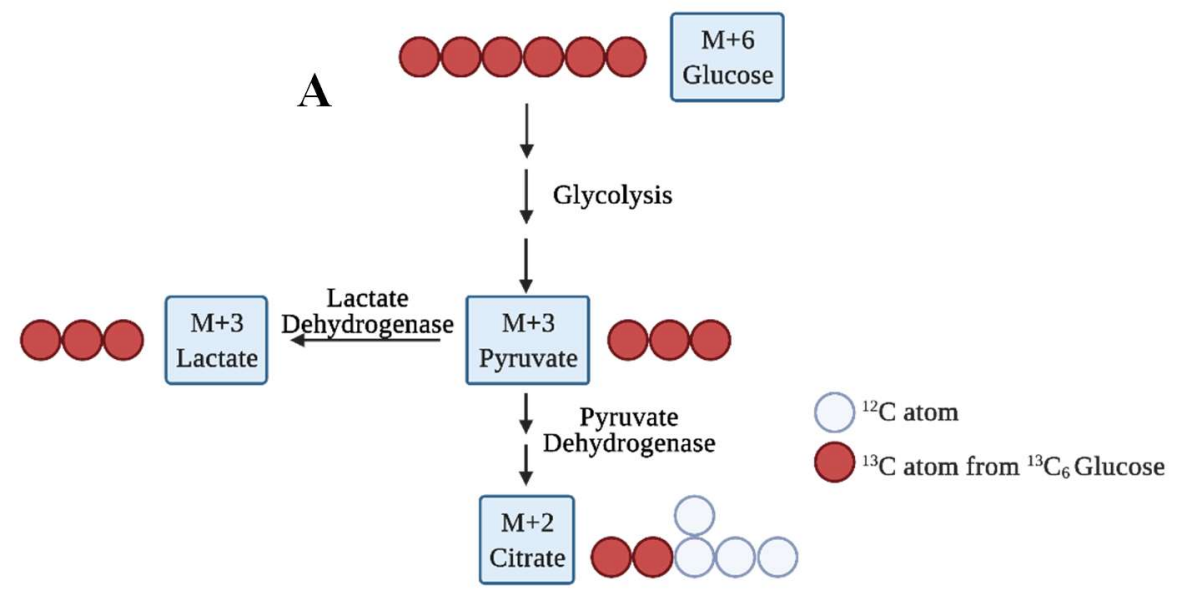

B

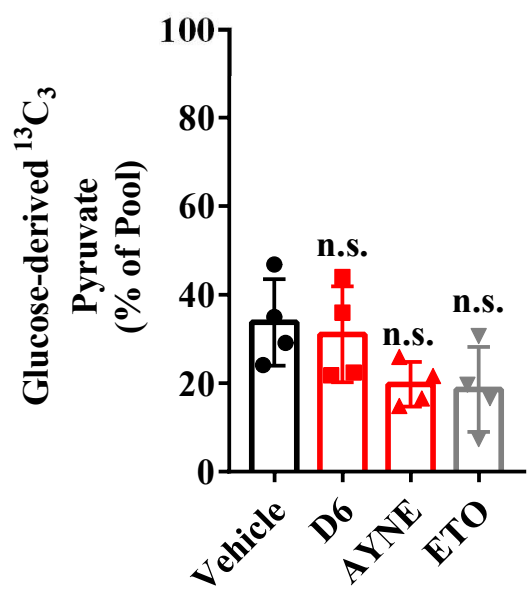

E

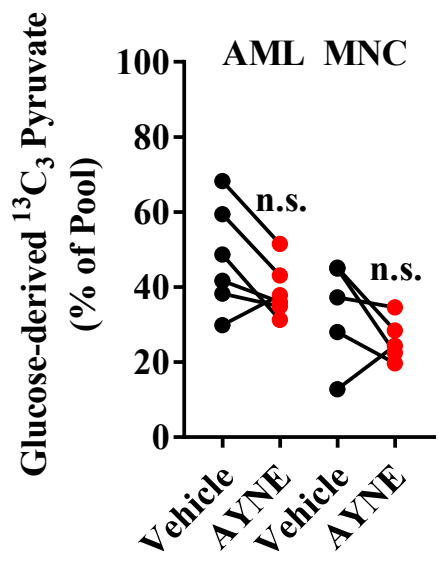

C

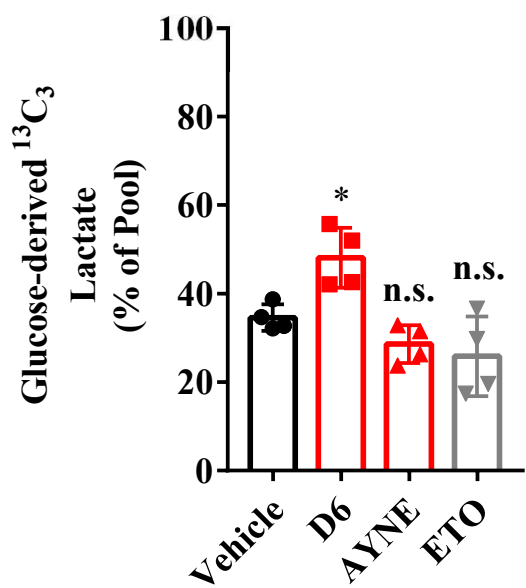

F

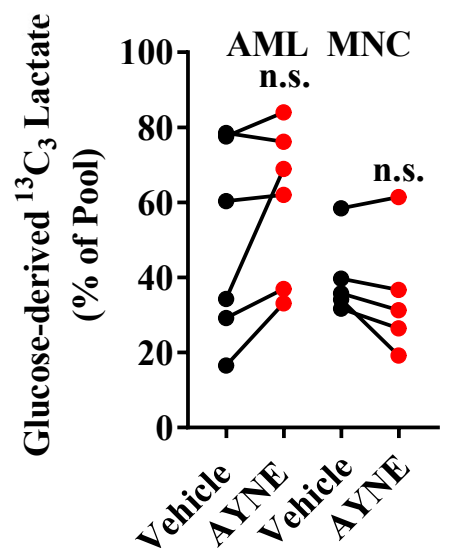

D

AML2

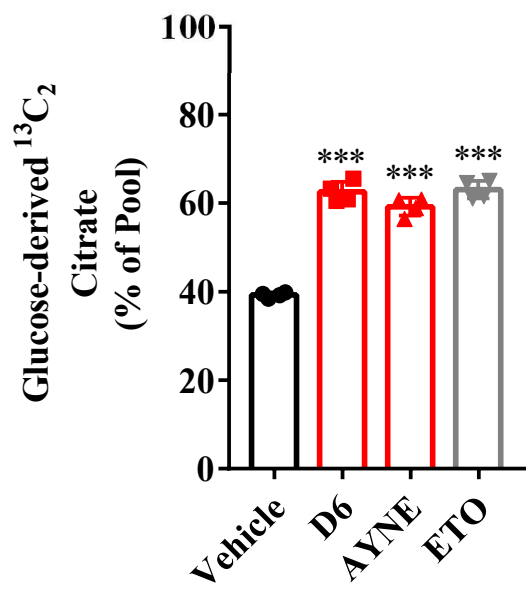

G

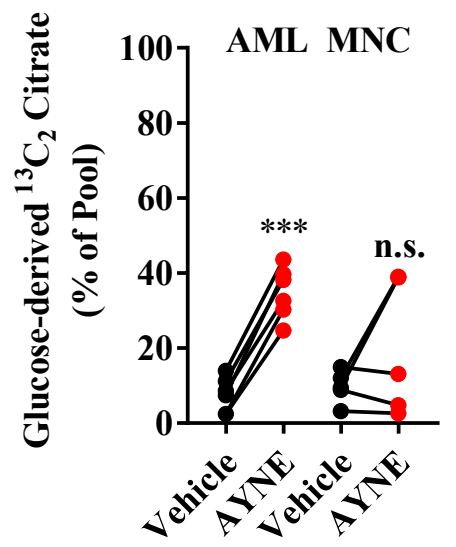


Figure 5.2.23 VLCAD inhibition triggers compensatory increase in PDH activity that is insufficient to alter overall glycolytic activity in TEX, AML2, and primary AML

(A) Schematic outlining the assessment of glycolytic activity with the ${ }^{13} \mathrm{C}_{6}$ (also $\left.\mathrm{M}+6\right)$ glucose tracer (Jones et al., 2018) The ${ }^{13} \mathrm{C}_{6}$ (also $\mathrm{M}+6$ ) glucose tracer is oxidized by glycolysis to pyruvate. Created in Biorender. Levels of (B) ${ }^{13} \mathrm{C}_{3}$ (also $\mathrm{M}+3$ ) pyruvate, $(\mathbf{C}){ }^{13} \mathrm{C}_{3}$ lactate, (D) ${ }^{13} \mathrm{C}_{2}$ (also $\mathrm{M}+2$ ) citrate in AML2 $(\mathrm{N}=4)$. Levels of $(\mathbf{E}){ }^{13} \mathrm{C}_{3}$ pyruvate, $(\mathbf{F}){ }^{13} \mathrm{C}_{3}$ lactate, $(\mathbf{G}){ }^{13} \mathrm{C}_{2}$ citrate in primary AML (AML\#3, AML\#15, AML\#16, AML\#19, AML\#25, AML\#26) and normal MNC samples $(\mathrm{N}=5)$. For (B-D), data presented as mean \pm S.D., n.s. not significant; $* \mathrm{p} \leq 0.05 ; * * \mathrm{p} \leq 0.002 ; * * *$ $\mathrm{p} \leq 0.001$, using a one-way ANOVA, Tukey's post-hoc test. For (E-G), data presented as mean \pm S.D., n.s. not significant; * $\mathrm{p} \leq 0.05 ; * * \mathrm{p} \leq 0.002 ; * * * \mathrm{p} \leq 0.001$, using a two-tailed, unpaired Student's $t$-test. For (B-G), AML2 cells were treated with either a DMSO vehicle (for parental AML2 and D6 cells) or $25 \mu \mathrm{M}$ AYNE or $100 \mu \mathrm{M}$ ETO. Primary AML and MNC samples were treated with either a DMSO vehicle or $50 \mu \mathrm{M}$ AYNE. All cell samples were treated for 12 hrs in the presence of the ${ }^{13} \mathrm{C}_{6}$ glucose tracer. Summary of patient cytogenetics are tabulated in Table 5.2.14. Summary of statistics are tabulated below in Table 5.2.12. MS analysis carried by E. Bohrnsen and Prof. A. D’Alessandro (University of Colorado Anschutz Medical Campus).

Table 5.2.12 Summary of statistics for data presented in Figure 5.2.23

\begin{tabular}{|c|c|c|c|c|c|c|}
\hline \multirow{3}{*}{ Isotopomer } & \multicolumn{6}{|c|}{ Cell Type } \\
\hline & \multicolumn{2}{|c|}{ AML2 } & \multicolumn{2}{|c|}{ Primary AML } & \multicolumn{2}{|c|}{ Normal MNC } \\
\hline & $\mathrm{F}(3,15)$ & $\mathrm{P}$-value & $\mathrm{t}(5)$ & P-value & $\mathrm{t}(4)$ & $\mathrm{P}$-value \\
\hline $\begin{array}{c}{ }^{13} \mathrm{C}_{3} \\
\text { Pyruvate }\end{array}$ & 2.899 & 0.08 & 2.07 & 0.09 & 1.307 & 0.26 \\
\hline $\begin{array}{c}{ }^{13} \mathrm{C}_{3} \\
\text { Lactate }\end{array}$ & 10.19 & 0.001 & 1.983 & 0.1 & 1.719 & 0.16 \\
\hline $\begin{array}{c}{ }^{13} \mathrm{C}_{2} \\
\text { Citrate }\end{array}$ & 148.9 & $<0.001$ & 18.42 & $<0.001$ & 1.336 & 0.25 \\
\hline
\end{tabular}




\subsubsection{VLCAD Inhibition Triggers Compensatory Increase In PDH Activity With No Significant Alteration In Glycolytic Activity In TEX, AML2, And Primary AML}

To assess the role of glycolytic metabolism upon FAO inhibition, the entry of glucosederived carbons from a ${ }^{13} \mathrm{C}_{6}$ glucose tracer into ${ }^{13} \mathrm{C}_{3}$ pyruvate, ${ }^{13} \mathrm{C}_{3}$ lactate, and ${ }^{13} \mathrm{C}_{2}$ citrate in AML2 (Figure 5.2.23 B-D), primary AML, and normal MNC samples (Figure 5.2.23 E-G) was assessed. Glycolysis-derived pyruvate, the terminal metabolite of glycolysis, has two major fates: lactate production, catalyzed by the lactate dehydrogenase enzyme in the cytosol, and oxidatively carboxylation, initiated by PDH on the mitochondrial surface (Figure 5.2.23 A) (Luengo et al., 2021) (Olson et al., 2016)(Jose et al., 2011). Across AML2 and primary AML, FAO inhibition at VLCAD by genetic knockdown or pharmacological blockade did not induce any changes in glucose-derived carbon enrichment into pyruvate (Figure 5.2.23 B, E).

Lactate production is also viewed as a measure of glycolytic activity coupled to the oxidation of NAD+ (Molina et al., 2018)(Lagadinou et al., 2013)(Sharon et al., 2019). ${ }^{13} \mathrm{C}_{3}$ lactate was increased slightly in AML2 cells with the VLCAD knockdown (Figure 5.2.23 C). However, FAO inhibition with AYNE and ETO did not alter enrichment of glucose-derived carbon into lactate with AML2 (Figure 5.2.23 C) and primary AML cells (Figure 5.2.23 F). In AML cells that are sensitive to VLCAD inhibition, the lack of change in glucose-derived carbon enrichment into both ${ }^{13} \mathrm{C}_{3}$ pyruvate and ${ }^{13} \mathrm{C}_{3}$ lactate confirmed no compensatory metabolic shift towards glycolytic activity in response to pharmacological VLCAD inhibition.

Levels of ${ }^{13} \mathrm{C}_{2}$ citrate from a ${ }^{13} \mathrm{C}_{6}$ glucose tracer is used as a surrogate measure of $\mathrm{PDH}$ activity (Kuntz et al., 2017). Genetic knockdown and pharmacological inhibition of VLCAD in AML2 (Figure 5.2.23 D) and primary AML (Figure 5.2.23 G) increased levels of ${ }^{13} \mathrm{C}_{2}$ citrate, confirming increased PDH activity following FAO inhibition at VLCAD. This increase in PDH 
activity was also observed earlier following VLCAD knockdown in AML2 and TEX cells (Figure 5.2.7) assessed with a commercial spectrophotometric kit. The lack of FAO inhibition observed in normal MNC samples, at the dose and timepoint that inhibited FAO in primary AML (Figure 5.2.21, 5.2.22), coincided with no changes in glycolytic activity (Figure 5.2.23 E-G). While normal MNC samples were spared, AML cells sensitive to FAO inhibition at VLCAD increased PDH activity, but not overall glycolytic activity, which was ultimately unable to prevent ATP depletion and cell death. These divergent metabolic fates between AML and MNC cells highlight the selective nature of VLCAD inhibition to kill AML but not normal hematopoietic cells, further demonstrating the pre-clinical efficacy of FAO inhibition at VLCAD in AML. 
A Inoculate mouse with AML patient or normal cord
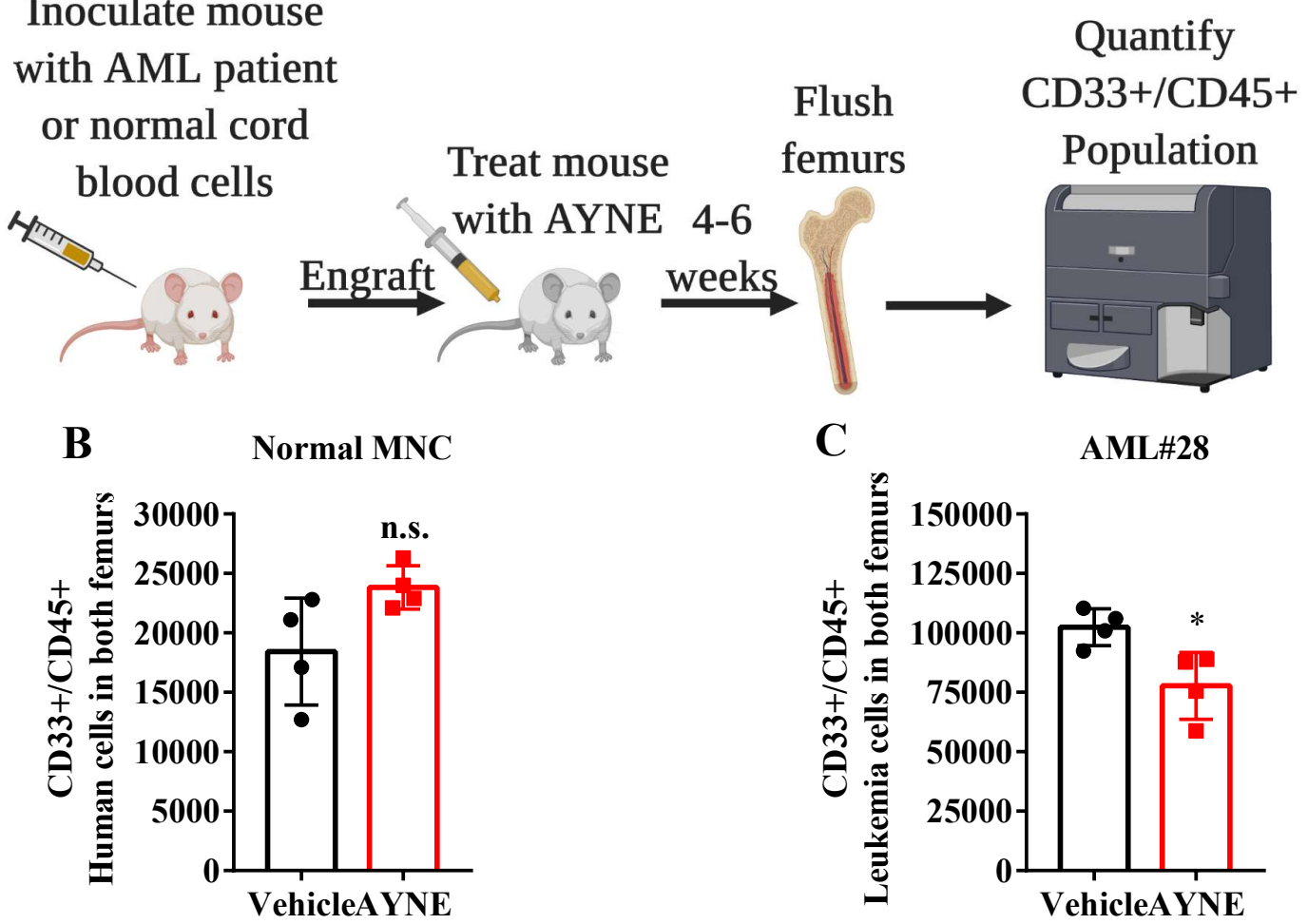

C

AML\#28

D

AML\#29
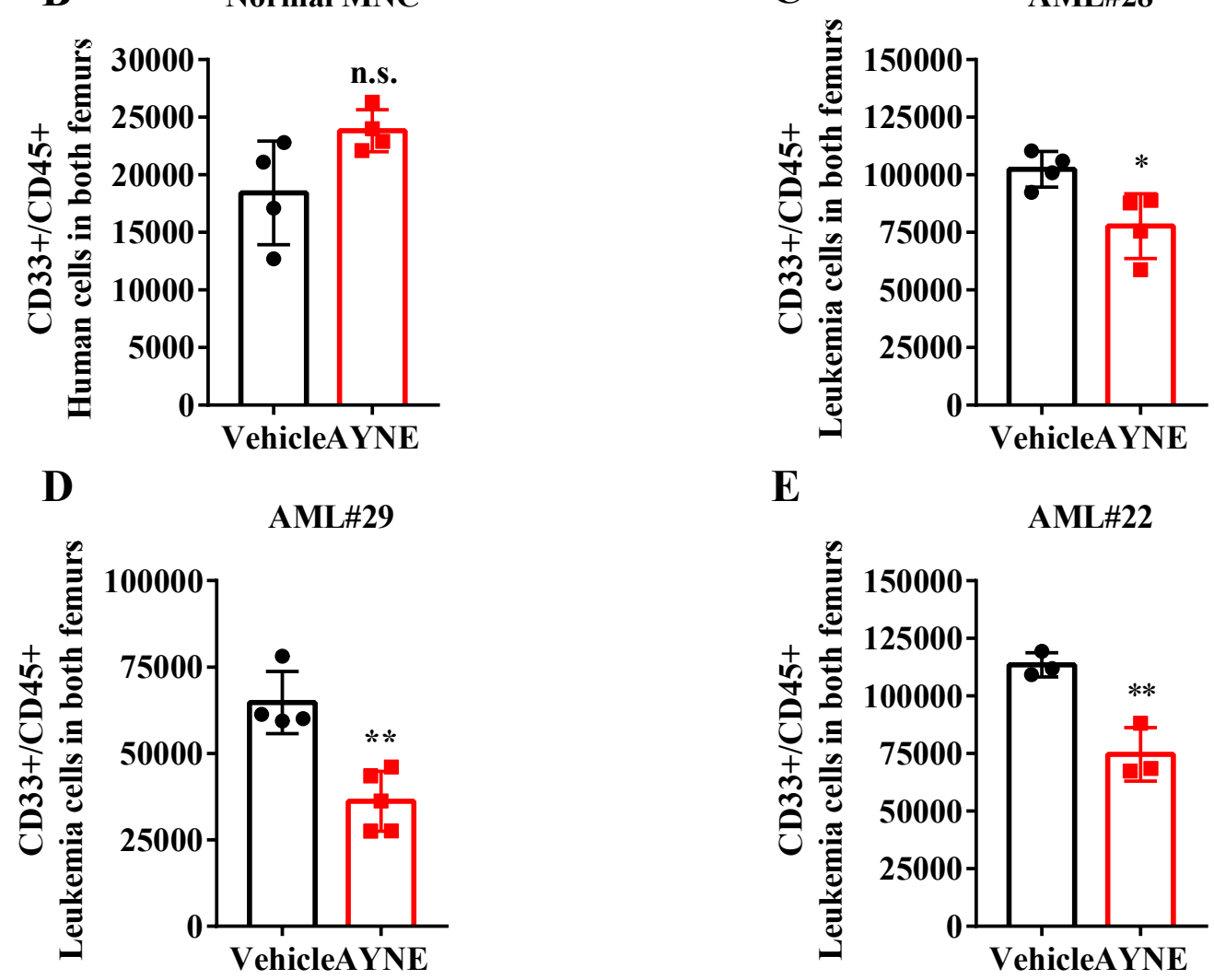

$\mathbf{E}$

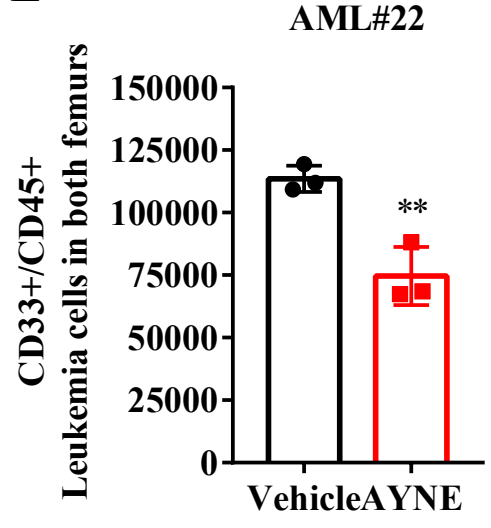

Figure 5.2.24 AYNE selectively induces death of the AML population, while sparing the normal blood cells in vivo

(A) Study schematic demonstrating the engraftment model to assess AYNE's anti-AML in vivo efficacy in NSG mice. Briefly, patient-derived AML or normal cord blood MNC cells were injected into tail veins of NSG mice. Human cells were given either a four or six week period to engraft into the femural bone marrow. Mice were then divided into two groups to receive either a vehicle emulsion (Ahmed et al., 2020) or an emulsion containing AYNE three times weekly for four or six weeks. Following sacrifice at the planned end of study, mice femurs were flushed, and human CD33+/CD45+ cells were quantified via flow cytometry. Created in Biorender. The engraftment period lasted one week for (B, C, D) and four weeks in (E). (B) Engraftment levels of healthy cord cells following treatment with $300 \mathrm{mg} / \mathrm{kg} /$ week AYNE or vehicle control for six weeks. Engraftment levels of patient-derived AML cells following treatment with either a vehicle 
or AYNE (C) $225 \mathrm{mg} / \mathrm{kg} /$ week or (D) $300 \mathrm{mg} / \mathrm{kg} /$ week for six weeks. (E) Engraftment of patientderived AML cells following treatment with either a vehicle or $200 \mathrm{mg} / \mathrm{kg} / \mathrm{week}$ AYNE for four weeks. For (B-E), data presented as mean \pm S.D., n.s. not significant; ${ }^{*} \mathrm{p} \leq 0.05$; ** $\mathrm{p} \leq 0.002$; *** $\mathrm{p} \leq 0.001$, using a Mann-Whitney U test. See Table 5.2.14 for patient cytogenetics. In vivo studies conducted by A. Roma and P. Jayanth. 


\subsubsection{AYNE Selectively Induces Death Of The AML Population, While Sparing The Normal Blood Cells In Vivo}

In vivo efficacy of AYNE was assessed with human patient-derived AML and donorderived hematopoietic populations in the NSG engraftment model, a well-established experiment used to assess potential therapeutics against human AML or normal hematopoietic subpopulations capable of engraftment (Figure 5.2.24 A). Following a one week engraftment period as well as a six week treatment period consisting of $300 \mathrm{mg} \mathrm{AYNE} / \mathrm{kg} /$ week, there was no significant change in CD33+/CD45+ hematopoietic cell engraftment (Figure 5.2.24 B: $\mathrm{p}=0.06$ ).

Human AML patient cells were given either one or four weeks to engraft followed by either a four or six week treatment period. During the treatment period, mice engrafted with human AML cells received either (Figure 5.2.24 C) 225, (Figure 5.2.24 D) 300, or (Figure 5.2.24 E) $200 \mathrm{mg}$ AYNE/kg/week. AYNE reduced AML engraftment following either 225 (Figure 5.2.24 C: $\mathrm{p}=0.03), 300$ (Figure 5.2.24 D: $\mathrm{p}=0.02)$, or $200 \mathrm{mg}$ AYNE/kg/week (Figure 5.2.24 E: $\mathrm{p}=0.005)$. Taken together, these results demonstrated the pre-clinical in vivo efficacy of selectively targeting the AML population with AYNE, a compound inhibiting long chain FAO at the VLCAD enzyme. 

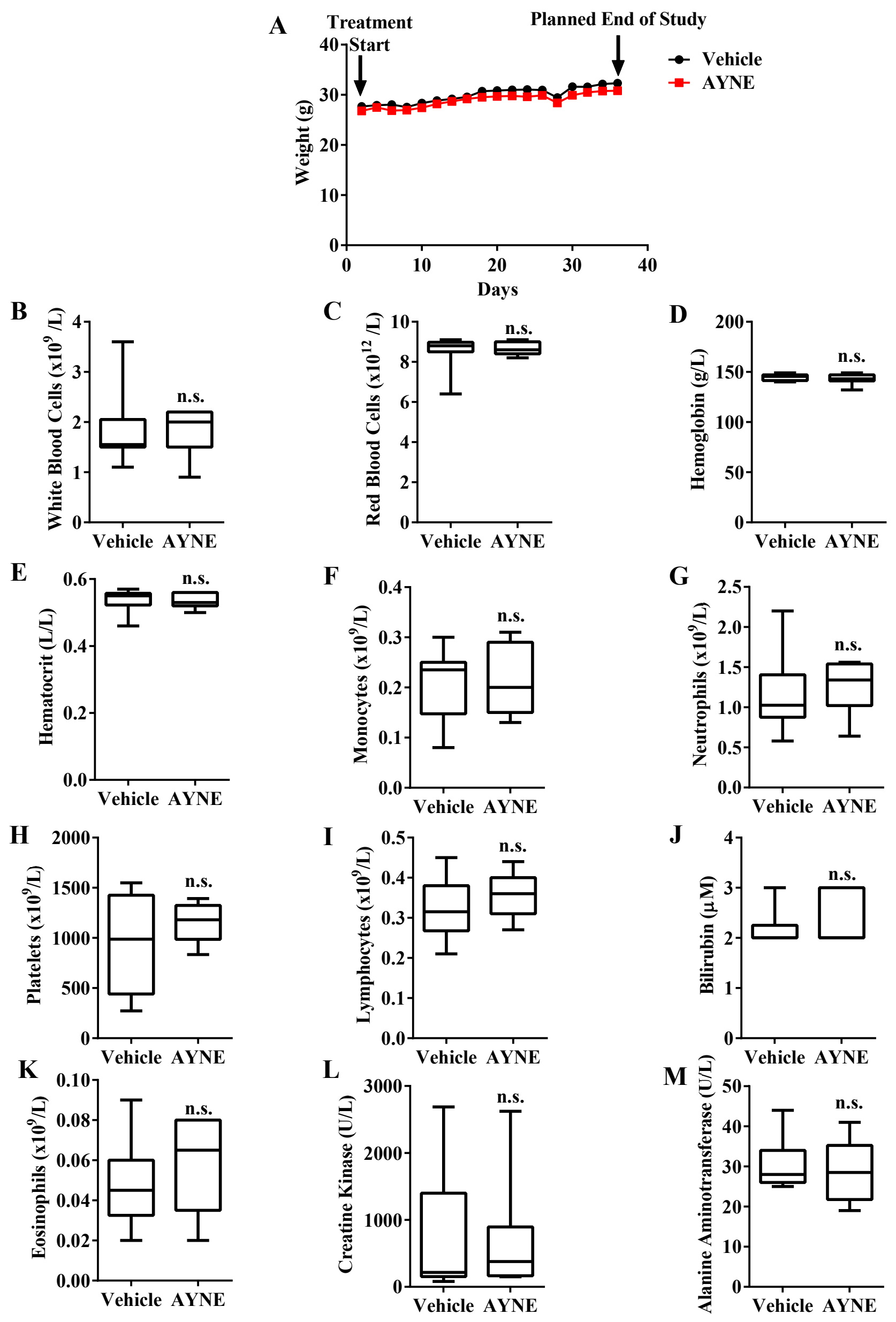
Figure 5.2.25 AYNE treatment is well tolerated by NSG mice.

Assessment and comparison of (A) body weight, (B) white blood cells, (C) red blood cells, (D) hemoglobin, (E) hematocrit, (F) monocytes, (G) neutrophils, (H) platelets, (I) lymphocytes, (J) bilirubin, (K) eosinophils, (L) creatine kinase activity, (M) alanine aminotransferase activity in mice receiving $300 \mathrm{mg} / \mathrm{kg} /$ week AYNE or a vehicle emulsion for six weeks ( $\mathrm{N}=4$ per group). Statistical summaries are tabulated below in Table 5.2.13. All data presented as mean \pm S.D., n.s. not significant; $* \mathrm{p} \leq 0.05 ; * * \mathrm{p} \leq 0.002 ; * * * \mathrm{p} \leq 0.001$, using a two-tailed, unpaired Student's $t$-test. Assessments carried out by the Animal Healthy Laboratory (University of Guelph).

Table 5.2.13 Summary of statistics for Figure 4.2.25.

\begin{tabular}{|c|c|}
\hline Biomarker & P-value \\
\hline Weight (g) & 0.19 \\
\hline White Blood Cells $\left(* 10^{\wedge} 9 / \mathrm{L}\right)$ & 0.82 \\
\hline Red Blood Cells $\left(* 10^{\wedge} 12 / \mathrm{L}\right)$ & 0.67 \\
\hline Hemoglobin $(\mathrm{g} / \mathrm{L})$ & 0.48 \\
\hline Hematocrit (L/L) & 0.54 \\
\hline Neutrophils $\left(* 10^{\wedge} 9 / \mathrm{L}\right)$ & 0.28 \\
\hline Monocytes (*10^9/L) & 0.85 \\
\hline Platelets $\left(* 10^{\wedge} 9 / \mathrm{L}\right)$ & 0.46 \\
\hline Lymphocytes $\left({ }^{*} 10^{\wedge} 9 / \mathrm{L}\right)$ & 0.38 \\
\hline Bilirubin $(\mu \mathbf{M})$ & 0.67 \\
\hline Eosinophils $\left({ }^{*} 10^{\wedge} 9 / \mathrm{L}\right)$ & 0.43 \\
\hline Creatine Kinase (U/L) & 0.54 \\
\hline Alanine Aminotransferase (U/L) & 0.65 \\
\hline
\end{tabular}




\subsubsection{AYNE Treatment Is Well Tolerated In NSG Mice}

Novel anti-AML therapeutics must be well tolerated by patients (Löwenberg et al., 2011). Current induction chemotherapeutics often adversely alter levels of blood and immune cells, as well as the activity of the creatine and alanine phosphatase enzymes, markers of liver and kidney function in patients (Shipley \& Butera, 2009). Analysis of complete blood counts, bilirubin, serological markers and mouse body weights showed no difference between control and AYNE

treatment groups (Figure 5.2.25 A-M), further demonstrating an anti-AML therapeutic dose of AYNE that is well-tolerated by mice. 


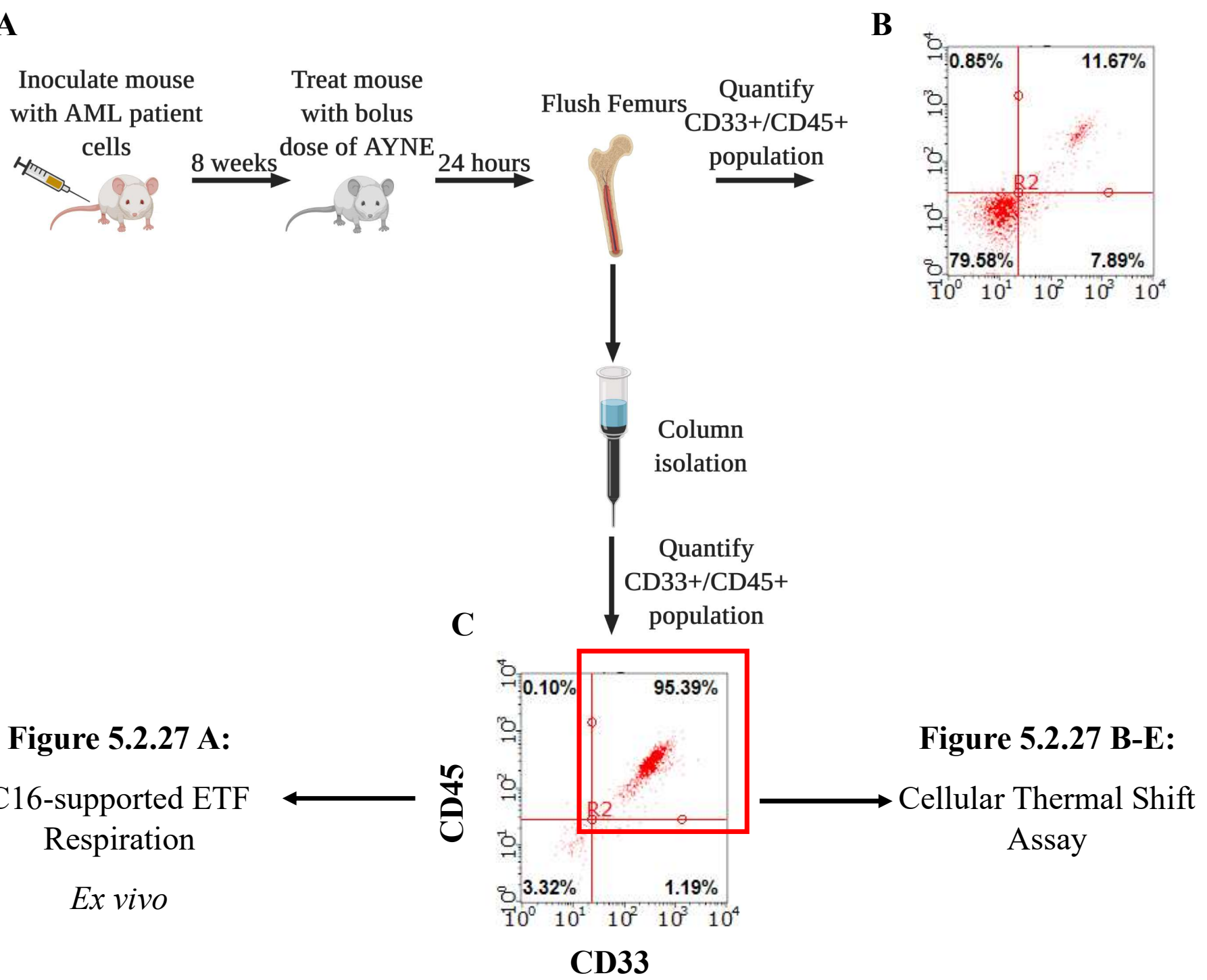

Figure 5.2.27 A:

C16-supported ETF Respiration

Ex vivo

Figure 5.2.26 Isolation of primary AML cells from mice treated AYNE

(A) Study schematic demonstrating injection of AML cells from AML\#28 into the tail vein of NSG mice. After six week engraftment period, mice were divided into three groups and received a one-time bolus dose of AYNE (150 or $200 \mathrm{mg} / \mathrm{kg}$ ) or a vehicle control emulsion (Ahmed et al., 2020). 24 hrs later, mice were sacrificed, femurs flushed, and a mix of human and mouse cells recovered. Abbreviations: $\mathrm{CD}$, cluster of differentiation. Created in Biorender. The mix of human and mice cells recovered from the femurs were quantified via flow cytometry (B) before and (C) after column purification with anti-CD33 and anti-CD45 antibodies. C16-supported ETF respiration and VLCAD thermo-stabilization (Below: Figure 5.2.27) were assessed with the isolated $\mathrm{CD} 33+/ \mathrm{CD} 45+$ human cells. Summary of patient cytogenetics are tabulated in Table 5.2.14. In vivo studies conducted by A. Roma and P. Jayanth. 


\subsubsection{Isolation Of Primary AML Cells From Mice Treated AYNE}

To assess in vivo binding of AYNE to VLCAD, AML cells from patient AML\#28 were engrafted and propagated in mice femurs for six weeks (Figure 5.2.26 A). Mice were divided into three groups and treated with either a vehicle emulsion or AYNE (150 or $200 \mathrm{mg} / \mathrm{kg}$ ) 24 hours prior to a planned sacrifice (Figure 5.2.26 A). A mix of human and mouse cells were recovered from the femurs (Figure 5.2.26 B). Column purification with magnetic beads coupled to an antiCD45 antibody (clone 5B1) isolated human cells from the human/mouse cell mixture (Figure 5.2.26 A). The purity of the isolated human cells was confirmed following staining with an antiCD33 antibody and an anti-CD45 antibody (clone H130) with a different epitope than the antiCD45 antibody used for column purification (Figure 5.2.26 C). Human cells ( $>95 \%$ purity) were then used in downstream experiments to demonstrate in vivo binding of AYNE to VLCAD (Figure 5.2.27). 
A

AYNE reduces

VLCAD-supported

ETF Respiration

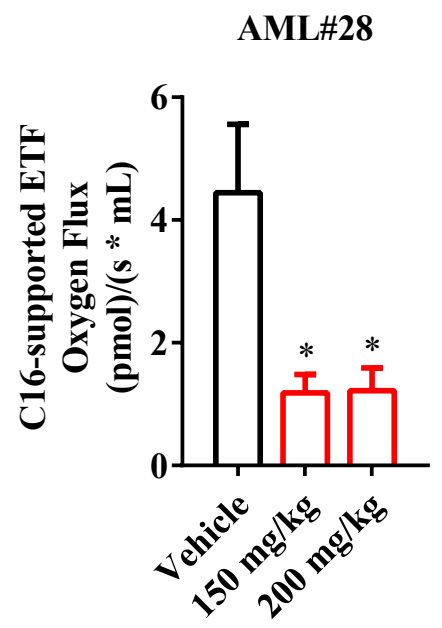

Cellular Thermal Shift Assay

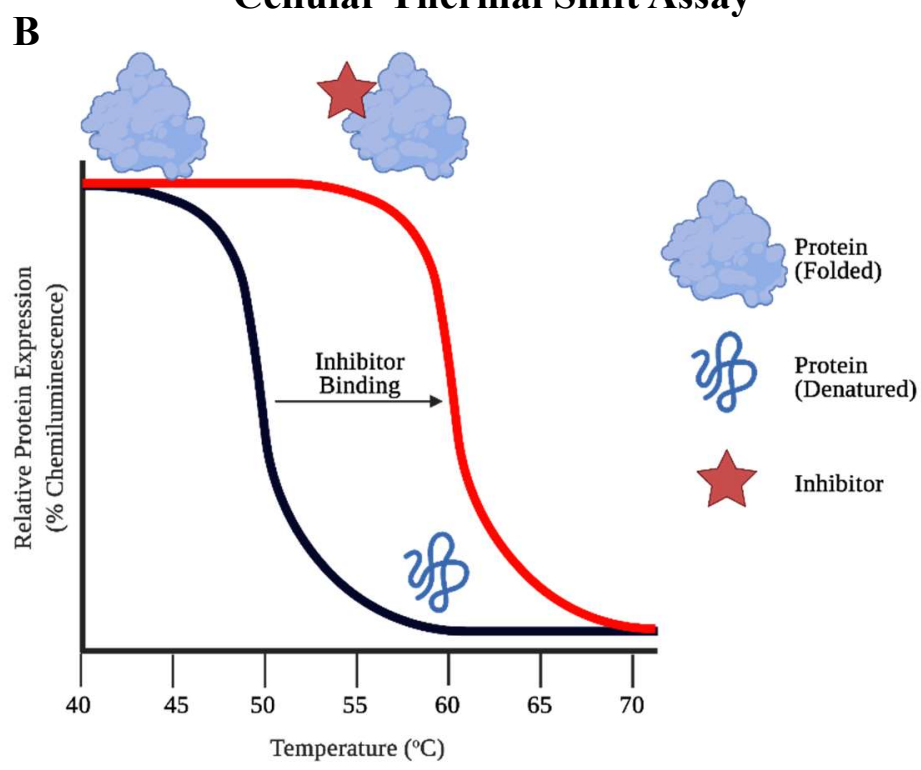

C

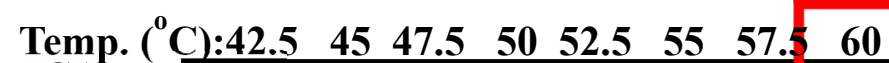

VLCAD

Beta Actin

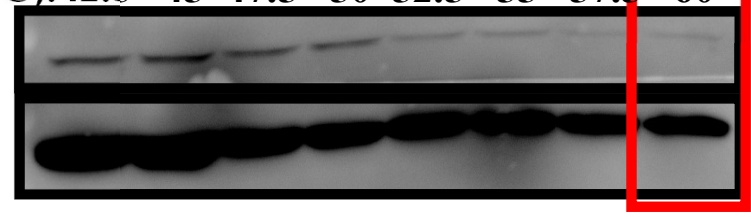

D AYNE (mg/kg)

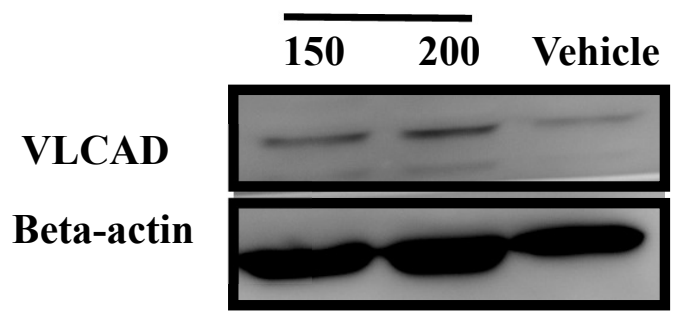

E AYNE stabilizes

VLCAD in vivo

AML\#28
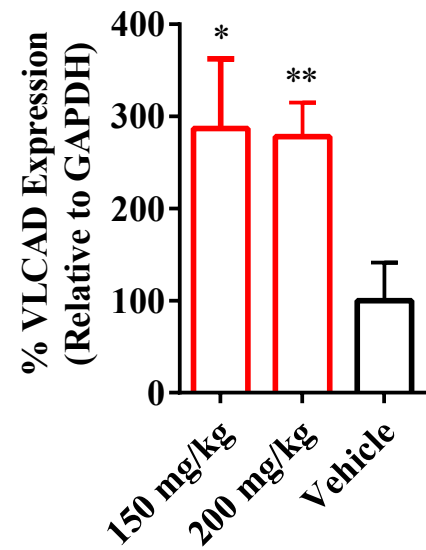
Figure 5.2.27 AYNE binds to and inhibits VLCAD in vivo

(A) Quantification of C16-supported ETF respiration of human cells from AML\#28 recovered from mice treated with either a vehicle emulsion (Ahmed et al., 2020) or an emulsion encapsulating AYNE (150 or $200 \mathrm{mg} / \mathrm{kg})(\mathrm{N}=3)$. (B) Schematic outlining thermal stabilization of a protein by ligand binding as assessed by cellular thermal shift assay (Ishii et al., 2017). In brief, lysate of cells treated with a potential inhibitor (AYNE) is subjected to a range of temperatures and centrifuged to remove heat denatured proteins. The binding of a potential inhibitor stabilizes the folded protein target at high temperatures, compared to a vehicle control. Black line shows the melting curve for a protein target from untreated cells. Red line shows a hypothetical melting curve for a protein from its folded state to its denatured state from cells treated with an inhibitor. Created in Biorender. (C) Ahead of cellular thermal shift assay with cells from AML\#28, cellular thermal shift analysis was conducted with lysate of untreated AML2 cells. (D) Western blot and (E) VLCAD densitometry from lysates of AML\#28 treated with either a vehicle emulsion or an emulsion containing AYNE following a $60^{\circ} \mathrm{C}$ heat shock $(\mathrm{N}=3)$. For $(\mathbf{A}, \mathbf{B}, \mathbf{C}, \mathbf{E})$, experiments conducted in triplicate. For $(\mathbf{A}, \mathbf{E})$, data presented as mean \pm S.D., n.s. not significant; ${ }^{*} \mathrm{p} \leq 0.05 ; * * \mathrm{p} \leq 0.002$; $* * * \mathrm{p} \leq 0.001$, using a one-way ANOVA, Tukey's post-hoc test. Summary of patient cytogenetics are tabulated in Table 5.2.14. 


\subsubsection{AYNE Binds To And Inhibits VLCAD In Vivo}

To verify VLCAD inhibition in vivo, primary patient-derived cells from AML\#28 were propagated in NSG mice and isolated for assessment of C16-supported ETF respiration, as an indirect measure of VLCAD activity (Figure 5.2.27 A), as well as the cellular thermal shift assay, as a measure of drug-target binding (Figure 5.2.27 B-E), following a one-time bolus dose of AYNE. Human leukemia cells ( $>95 \%$ pure) from AML\#28 recovered from NSG mice treated with AYNE showed significant inhibition of ETF respiration (Figure 5.2.27 A: $F(3,7)=13.29, p=0.01$ ).

In brief, the cellular thermal shift assay involves performing a heat shock to cell lysate treated with either a vehicle control or AYNE, the ligand of interest. A ligand, potentially a chemical inhibitor, will bind to and stabilize the enzyme target. Densitometry of the lysate after prolonged high-speed centrifugation allows western blot quantification of the stabilized enzyme, confirming in vivo target engagement. Prior to the cellular thermal shift assay with primary leukemia cells, the melting point of VLCAD was determined to be $60^{\circ} \mathrm{C}$ with AML2 cells (Figure 5.2.27 C), as noted by the lack of VLCAD in the lysate after a $60^{\circ} \mathrm{C}$ heat shock. AYNE treatment of mice carrying leukemia cells from AML\#28 demonstrated a shift in VLCAD melting temperature, which confirmed an AYNE-VLCAD interaction in vivo, (Figure 5.2.27: $\mathrm{F}(3,8)=14.95, \mathrm{p}=0.005)$. 
Table 5.2.14 Cytogenetics of patient IDs used in chapter 5

\begin{tabular}{|c|c|}
\hline $\begin{array}{c}\text { Patient } \\
\text { ID }\end{array}$ & Cytogenetics \\
\hline AML\#1 & Unsuccessful \\
\hline AML\#2 & $46, \mathrm{XY}[20]$ \\
\hline AML\#3 & $46, \mathrm{XX}[20]$ \\
\hline AML\#4 & $\begin{array}{c}45 \sim 46, X, \operatorname{add}(\mathrm{X})(\mathrm{q} 22),-2,-4, \operatorname{add}(7)(\mathrm{q} 31) \\
, \operatorname{der}(19) \mathrm{t}(2 ; 19)(\mathrm{q} 11.2 ; \mathrm{q} 13.1) \\
, \operatorname{add}(20)(\mathrm{q} 11.2),+1 \sim 2 \mathrm{mar}[\mathrm{cp} 9]\end{array}$ \\
\hline AML\#5 & Unsuccessful \\
\hline AML\#6 & $46, X Y[20]$ \\
\hline AML\#7 & $46, \mathrm{XX}, \mathrm{t}(9 ; 22)(\mathrm{q} 34 ; \mathrm{q} 11.2)[20]$ \\
\hline AML\#8 & $49, \mathrm{XY},+12,+16,+21[10]$ \\
\hline AML\#9 & $46, X Y[20]$ \\
\hline AML\#10 & $46, X Y, \operatorname{inv}(16)(p 13.1 q 22)$ \\
\hline AML\#11 & INV (16) \\
\hline AML\#12 & $46, X Y[20]$ \\
\hline AML\#13 & $46, \mathrm{XX}[20]$ \\
\hline AML\#14 & $46, X Y[20]$ \\
\hline AML\#15 & $47, \mathrm{XY},+\mathrm{X}[20]$ \\
\hline AML\#16 & $45, X,-Y[18] / 46, X Y[2]$ \\
\hline AML\#17 & $46, \mathrm{XY}[20]$ \\
\hline AML\#18 & $46, \mathrm{XY},+8[9]$ \\
\hline
\end{tabular}




\begin{tabular}{|c|c|}
\hline AML\#19 & $\begin{array}{c}51 \sim 52, \mathrm{X}, \operatorname{idic}(\mathrm{Y})(\mathrm{q} 11.2) \times 6 \sim 7[\mathrm{cp} 2] / 46, \mathrm{XY}[3] . \operatorname{ish} \operatorname{idic}(\mathrm{Y}) \\
(\mathrm{q} 11.2)(\mathrm{DYZ3}++) \times 6 \sim 7[2]\end{array}$ \\
\hline AML\#20 & 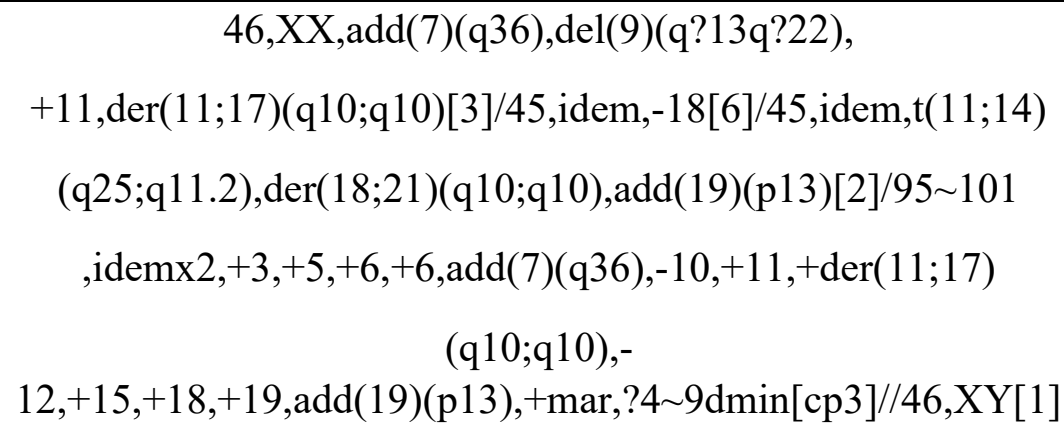 \\
\hline AML\#21 & $46, X Y[20]$ \\
\hline AML\#22 & $46, \mathrm{XX}[12]$ \\
\hline AML\#23 & $48, \mathrm{XX},+8,+20[16] / 46, \mathrm{XX}[4]$ \\
\hline AML\#24 & $46, \mathrm{XX}, \mathrm{t}(8 ; 21)(\mathrm{q} 22 ; \mathrm{q} 22)[8]$ \\
\hline AML\#25 & $46, X X[20]$ \\
\hline AML\#26 & $48, \mathrm{XY}, \operatorname{add}(1)(\mathrm{q} 32),+\operatorname{del}(5)(\mathrm{q} 12 \mathrm{q} 33),+\operatorname{mar}[4] / 46, \mathrm{XY}[7]$ \\
\hline AML\#27 & $46, X X[20]$ \\
\hline AML\#28 & $46, \mathrm{XX}[12]$ \\
\hline AML\#29 & $45, \mathrm{XX},-4,-9,-21,+\operatorname{mar} 1,+\operatorname{mar} 2[5] / 46, \mathrm{XX}[1]$ \\
\hline
\end{tabular}




\subsection{Discussion}

The results presented in this chapter investigated the hypotheses set out in chapter 2 to identify that VLCAD, an intramitochondrial enzyme catalyzing the first reaction of long chain FAO, was overexpressed in AML, compared to normal hematopoiesis, and critical to mitochondrial oxidative metabolism. In agreement with previous studies demonstrating hindered AML progression following the loss of APOC2 (Zhang et al., 2020)/CD36(Ye et al., 2016), CPT1 (Shafat et al., 2017)(Farge et al., 2017), CT2 (Wu et al., 2015), FABP4 (Shafat et al., 2017), PML(Ito et al., 2008), genetic ablation of VLCAD also resulted in reduced proliferation rates, clonogenic potential, and engraftment capacity in AML, while normal cells were spared. Given that no widely available research or clinical grade VLCAD chemical inhibitors exist, a HRR screen identified AYNE, an avocado-derived tri-hydroxylated fatty alcohol with a terminal triple bond, as an inhibitor of VLCAD activity. In AML lines and primary patient samples, the loss of VLCAD activity, by genetic ablation or pharmacological inhibition, inhibited FAO, decreased contribution

of fat-derived carbon into the TCA cycle, failed to trigger compensatory glycolytic activity, ultimately resulting in cell death.

In contrast, FAO inhibition in normal hematopoietic cells did not alter TCA metabolism or trigger an energy crisis. Compared to the malignant leukemia population, the sparing of normal hematopoietic cells is in agreement with previous studies. The bulk normal population exhibited lower rates of FAO activity, due to lower gene expression of critical FAO enzymes, and are less reliant on FAO as a pro-survival pathway (Samudio et al., 2010)(Ito et al., 2008). In line with these observations, this study demonstrated that VLCAD gene and protein expression in normal MNCs were lower, compared to AML, in publicly available gene expression databases and primary patient-derived samples. 
To further demonstrate a therapeutic window for VLCAD inhibition in AML, clinical symptoms of VLCAD deficiency, a rare human metabolic disease characterized by a mutation in the $A C A D V L$ gene results in decreased VLCAD activity, should be considered (Wanders et al., 2010). Mutations causing VLCAD deficiency are highly heterogeneous and may result in sudden death in newborns while human adults may be asymptomatic (Vockley et al., 2016). Tissuespecific clinical symptoms in humans manifest due to abnormal mitochondrial dysfunction and energy metabolism in three cell types dependent on FAO: first, cardiomyopathy in the heart, hypoketotic hyperglycemia in the liver, and rhabdomyolysis in skeletal muscle (Schiff et al., 2013). VLCAD knockout studies in mice and the clinical symptoms of VLCAD deficiency in human adults confirmed that the complete loss of VLCAD activity can be managed by consuming frequent meals with medium chain fats and avoiding a diet high in long chain fats, fasting, cold exposure, and strenuous physical activity (Xiong et al., 2013)(Vockley et al., 2016). While future studies in these cell types are required to confirm the lack of adverse effects with anti-AML doses of AYNE, mice treated with AYNE in the engraftment studies presented in this chapter showed no abnormal cardiac, hepatic, or skeletal muscle tissue upon visual inspection at endpoint.

The capacity to switch to glycolysis-linked lactate production upon inhibition of mitochondrial oxidative metabolism at various mitochondrial targets determines AML survival (Molina et al., 2018)(Lagadinou et al., 2013)(Sharon et al., 2019). Metabolic flexibility varies between AML cell lines and between subpopulations within a single AML patient, reflecting the heterogeneity of AML. Previously, normal hematopoietic cells exhibited a high capacity to switch to and upregulate lactate production, further demonstrating a therapeutic window to target mitochondrial metabolism in AML therapy (Lagadinou et al., 2013). Upregulation of glycolysislinked lactate production compensates for the inhibition of mitochondrial oxidative metabolism 
through the kinetically favorable production $\mathrm{ATP}$, regeneration of $\mathrm{NAD}^{+}$, and production of biosynthetic metabolites to support cell survival and proliferation (Luengo et al., 2021)(Olson et al., 2016)(Jose et al., 2011). While FAO coupled to OXPHOS produces larger amounts of ATP, glycolysis-linked ATP production is kinetically favorable and is critical to preventing ATP depletion under metabolic or pharmacological stress. Increased glycolytic flux generates higher levels of glycolytic intermediates, which can be shunted to adjacent pathways to fuel cell growth, and increases regeneration of $\mathrm{NAD}^{+}$, a critical cofactor in the production of biosynthetic metabolites including amino acids, protein, lipids, and nucleosides (Lunt \& Vander Heiden, 2011)(Luengo et al., 2021)(Jose et al., 2011). To further demonstrate that cell fate is dependent on compensatory glycolysis, avo-B previously induced death of the AML line THP-1 as a single agent but was unable to induce AML death alone in the AML line MOLM-13. Glycolytic metabolite profiling of MOLM-13 demonstrated a marked increase in glycolytic intermediates and lactate, confirming that activation of compensatory glycolysis upon FAO inhibition prevented AML death. Inhibition of compensatory glycolysis by 2-deoxyglucose in combination with avo-B was required to kill MOLM-13 cells (Tabe et al., 2018).

In this chapter, glucose-derived pyruvate and lactate levels were not altered in response to loss of VLCAD activity, confirming no compensatory upregulation of glycolysis in primary patient samples preceding AML death. The decreased proliferation rate and engraftment capacity of TEX and AML2 lacking VLCAD are in agreement with previous studies demonstrating that increased $\mathrm{PDH}$ activity further depleted cellular $\mathrm{NAD}^{+}$, a critical metabolite required for cell proliferation, and increased oxidative stress, resulting in suppressed engraftment capacity. Taken together, AML cells that were sensitive to pharmacological inhibition or genetic ablation of VLCAD did not sufficiently upregulate glycolysis-linked to lactate production and, instead, upregulated PDH 
activity, previously shown to be detrimental to AML proliferation and engraftment (Wang et al., 2014)(Hao et al., 2019).

In addition to compensatory metabolism, inhibition of mitochondrial oxidative metabolism could be rescued by supplementing the starved leukemia cells with a metabolite downstream to the point of inhibition. In AML, the inhibition of glutaminolysis by the pharmacological inhibitor CB-839 (Jacque et al., 2015) or L-asparaginase (Jun et al., 2011) could be attenuated by supplementing the starved cells with exogenous $\alpha$-ketoglutarate or oxaloacetate, resulting in the partial restoration of TCA cycling and ATP levels. Similarly in this study, supplementation with sodium heptanoate, a medium chain length fat that bypasses the blockage at VLCAD to be oxidized by medium chain FAO enzymes (Vockley et al., 2016), in AML cells treated with AYNE averted cell death by partial restoring FAO activity. This is in agreement with a previous study where supplementation with the medium length fat sodium octanoate in AML cells treated with the CPT1 inhibitor ETO attenuated cell death (Stevens et al., 2020). Taken together, this reduction of AML cytotoxicity by the supplementation of a downstream metabolite of the inhibited pathway further demonstrated that long chain FAO was the primary metabolic target of VLCAD knockdown and pharmacological inhibition by AYNE.

In summary, the results presented in this chapter are in agreement with previous studies (Samudio et al., 2010)(Shafat et al., 2017)(Zhang et al., 2020)(Wu et al., 2015)(Farge et al., 2017)(Ito et al., 2008) demonstrating a critical role for the FAO pathway in AML. Chapter 5 established VLCAD as a novel target that is overexpressed in AML mitochondrial metabolism, compared to normal hematopoietic cells, and conducted a thorough pharmacodynamic study of AYNE, a novel VLCAD chemical inhibitor that is well-tolerated in pre-clinical engraftment studies. 


\subsection{Chapter 6 A Structure-Activity Study Of Avocadyne (AYNE)'s Anti-}

\section{AML Mechanism Of Action}

Matthew Tcheng1, Vitor L.S. Cunha ${ }^{2}$, Nawaz Ahmed ${ }^{1}$, Xiaofan Liu ${ }^{3}$, Richard W. Smith ${ }^{4}$, Angelo D’Alessandro $^{5}$, Mark D. Minden ${ }^{6}$, Jerry Vockley ${ }^{7}$, George A. O’Doherty ${ }^{3}$, Todd L. Lowary ${ }^{2,8}$, and Paul A. Spagnuolo ${ }^{1 *}$

${ }^{1}$ Department of Food Science, University of Guelph, 50 Stone Rd. Guelph Ontario, Canada. N1G2W1

${ }^{2}$ Department of Chemistry, University of Alberta, 4-008 Centennial Centre for Interdisciplinary Science, Edmonton, AB, Canada. T6G 2G2

${ }^{3}$ Department of Biochemistry and Chemical Biology, Northeastern University, 360 Huntington Avenue, Boston, MA, USA 02115

${ }^{4}$ University of Waterloo Mass Spectrometry Facility, Department of Chemistry, 200 University Avenue West, Waterloo, ON, N2L 3G1, Canada

${ }^{5}$ Department of Biochemistry and Molecular Genetics, University of Colorado Anschutz Medical Campus, Aurora, CO, USA 80045

${ }^{6}$ Princess Margaret Cancer Center, Ontario Cancer Institute, 610 University Ave, Toronto ON, M5G 2M9, Canada;

${ }^{7}$ Department of Pediatrics and Center for Rare Disease Therapy, UPMC, Children's Hospital of Pittsburgh, University of Pittsburgh, Pittsburgh, PA 15224 \& Department of Human Genetics, School of Public Health, University of Pittsburgh, Pittsburgh, PA 15260

${ }^{8}$ Institute of Biological Chemistry, Academia Sinica, Academia Road, Section 2, \#128, Nangang, Taipei, Taiwan, 11529

* Correspondence email: paul.spagnuolo@uoguelph.ca

Modified version of this manuscript has been accepted at the Journal of Food \& Function under the title "Structure-activity relationship of avocadyne" 


\subsection{Introduction}

The constituents of the anti-AML mixture avo-B are acetogenins, aliphatic fatty alcohols either 17,19 , or 21 carbon atoms in a linear aliphatic chain with diverse structural modifications (Salazar-López et al., 2020)(Rodríguez-López et al., 2017). Alteration of acetogenin length, stereochemistry, and the presence and position of its unsaturations and functional groups result in a wide spectrum of bioactivities (Brooke et al., 2011)(Néeman et al., 1970).

To further define the mechanism of AYNE established in chapter 5, structure-activity studies with the components of avo-B are required. The structure of both avo-B constituents were first elucidated by Kashman (Figure 2.1.1)(Kashman et al., 1969); further structure elucidation studies reported the $(2 R, 4 R)$-stereoisomer of AENE was extracted from avocadoes (Sugiyama et al., 1982)(Oberlies et al., 1998). The ability of AYNE and AENE to kill or inhibit the growth of several gram-positive bacteria (Bacillus subtilis NCTC 3610, Bacillus cereus NCTC 7587 Staphylococcus aureus oxford, Saccharomyces cerevisiae ATCC 7752) and two strains of yeast (Saccharomyces cerevisiae ATCC 7752, Saccharomyces cerevisiae S288 C) was also assessed. AENE was the most potent bacteriocidal compound, yielding disc inhibition zones of 10-40 mm with a minimum inhibitory concentration of $2-31 \mu \mathrm{g} / \mathrm{mL}$ against the microorganisms tested (Néeman et al., 1970). Despite inducing AML cytotoxicity (Lee et al., 2015), AYNE only slowed the growth of and failed to kill the microorganisms tested. Loss of the terminal unsaturation or esterification of an acetyl moiety at either $C-1, C-2, C-4$ in AENE or AYNE resulted in complete attenuation of bacteriocidal activity by AENE and bacteriostatic activity by AYNE, confirming that structure is critical to acetogenin bioactivity (Néeman et al., 1970).

A 95\% ethanol extract of the edible avocado pulp contained AYNE, AENE, and 1,2,4trihydroxynonadecane, a 19-carbon long full acetogenin with $(2 R, 4 R)$-stereochemistry but no 
terminal unsaturation. All three compounds as single agents were similarly potent and demonstrated $\mathrm{IC}_{50}$ values between 1-5 $\mu \mathrm{g} / \mathrm{mL}$ against the human lung carcinoma A-549, mammary adenocarcinoma MCF-7, colon adenocarcinoma HT-29, kidney carcinoma A-498 cell lines. The three individual compounds were most cytotoxic against the prostate adenocarcinoma cells. AYNE, AENE, and the saturated 19 carbon long acetogenin demonstrated $\mathrm{IC}_{50}$ values of $6.1^{*} 10^{-}$ ${ }^{2}, 4.6^{*} 10^{-1}, 1.2 \mu \mathrm{g} / \mathrm{mL}$, respectively, against PC-3. This stark difference in cytotoxicity, despite only slight differences in unsaturation and aliphatic linear chain length, further underscores that acetogenin bioactivity in different disease states is highly dependent on structure. Interestingly, the mixture of all three compounds demonstrated higher $\mathrm{IC}_{50}$ values, compared to these three similarly structured compounds as single agents. This is in line with the antagonism observed with avo-B-induced AML cytotoxicity (Oberlies et al., 1998) but underscores the importance of determining a structure-activity relationship in specific disease states.

In terms of anti-AML acetogenin activity, avocatin- $\mathrm{A}$ is a similarly structured 1:1 mixture containing acetylated analogies of AYNE and AENE; acetylation at $C-1$ attenuated anti-AML efficacy (Lee et al., 2015). In chapter $4 \& 5$, AYNE was the most potent FAO inhibitor and cytotoxic component of the avo-B mixture, suggesting that the terminal triple bond is critical to inhibition at VLCAD. The role of the structural components of AYNE and AENE in their ability to inhibit fat-supported mitochondrial respiration and induce AML death have not been previously investigated in detail. 


\subsection{Results}
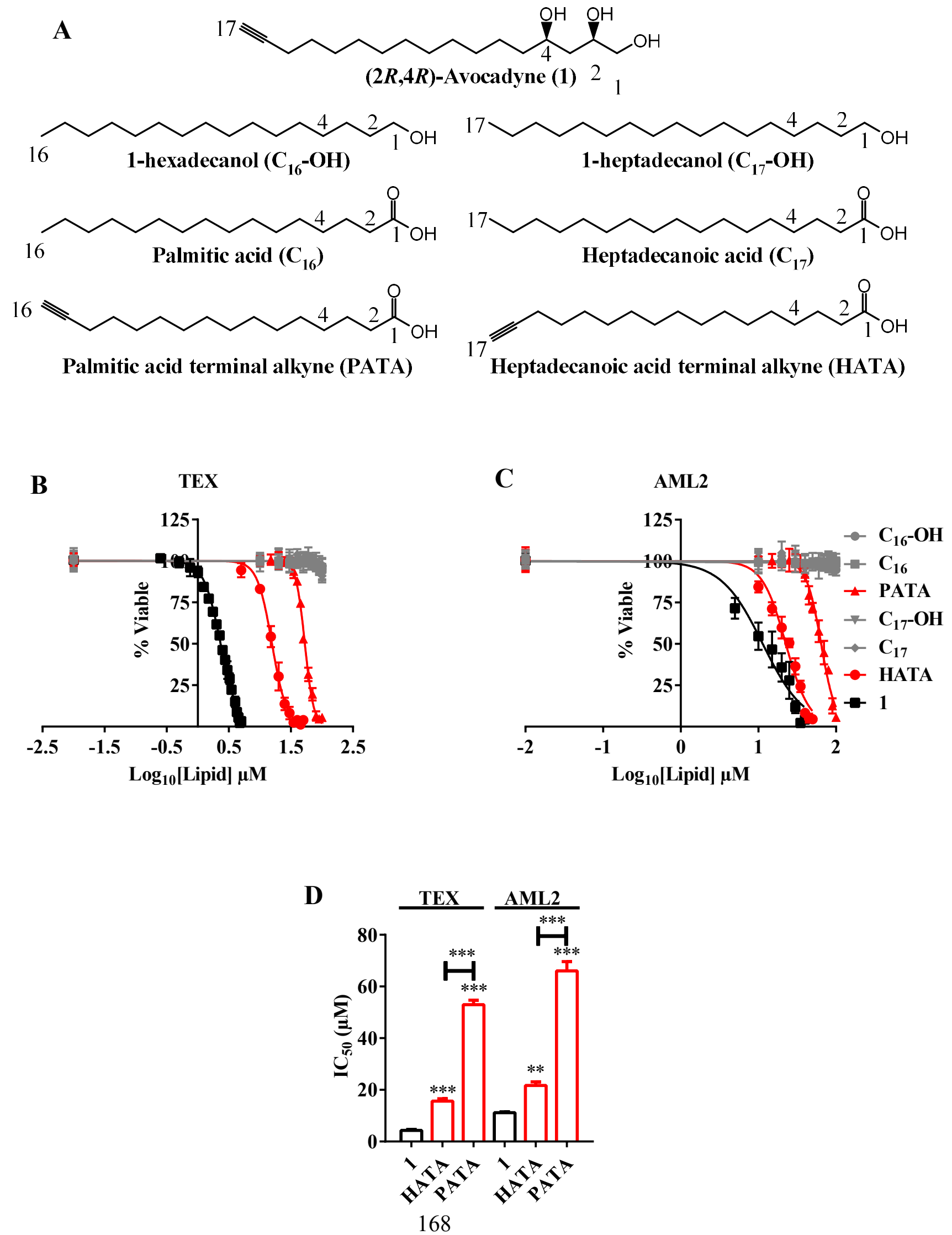
Figure 6.2.1 AYNE's Terminal Triple Bond And An Odd Number Of Carbon Atoms Are Critical To Its AML Cytotoxicity.

(A) Chemical structures of (2R,4R)-AYNE 1 (Synthesized by (Cunha et al., 2019)), 1-hexadecanol $\left(\mathrm{C}_{16}-\mathrm{OH}\right), 1$-hexadecanoic acid $\left(\mathrm{C}_{16}\right)$, palmitic acid terminal alkyne (PATA), 1-heptadecanol $\left(\mathrm{C}_{17-}\right.$ $\mathrm{OH})$, 1-heptadecanoic acid $\left(\mathrm{C}_{17}\right)$, and heptadecanoic acid terminal alkyne (HATA). Numbers underneath the chemical structures highlight compound length and $C-1, C-2, C-4$, the positions of the hydroxyl groups in AYNE 1. Chemical structures drawn in ChemDraw 20.0. Cell viability of (B) TEX and (C) AML2 following a 72-hour treatment analyzed with flow cytometry with a 7AAD stain ( $\mathrm{N}=3$ ). (D) Comparison of HATA, PATA, and AYNE $1 \mathrm{IC}_{50}$ values for TEX and AML2 which are tabulated below in Table 6.2.1 and were calculated using dose response inhibition equation on GraphPad Prism 7.0. HATA and PATA $\mathrm{IC}_{50}$ values were the compared to 1 for TEX and AML2 (N=3). For (D), IC I0 $_{50}$ values of HATA and PATA calculated from (C) viability data is presented and compared to AYNE 1 as mean \pm S.D., ${ }^{*} p<0.05, * * p<0.01$, $* * * \mathrm{p}<0.001$ using an unpaired, one-tailed, student's $t$-tests or ANOVA were calculated in GraphPad Prism 7.0.

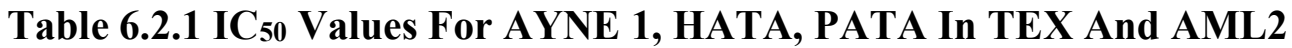

\begin{tabular}{|c|c|c|c|}
\hline Compound & AYNE $(\boldsymbol{\mu M})$ & HATA $(\boldsymbol{\mu M})$ & PATA $(\boldsymbol{\mu M})$ \\
\hline $\begin{array}{c}\text { TEX IC50 (Mean } \pm \\
\text { STDEV) }\end{array}$ & $2.33 \pm 0.10$ & $15.65 \pm 0.57^{* * *}$ & $52.93 \pm 0.66^{* * *}$ \\
\hline $\begin{array}{c}\text { AML2 IC50 (Mean } \pm \\
\text { STDEV) }\end{array}$ & $11.41 \pm 1.87$ & $22.60 \pm 1.37^{* *}$ & $64.44 \pm 3.63^{* * *}$ \\
\hline
\end{tabular}




\subsubsection{AYNE's Terminal Triple Bond And An Odd Number Of Carbon Atoms Are Critical To Its AML Cytotoxicity}

To assess the roles of the hydroxyl and terminal alkyne moieties as well as the aliphatic chain length in AYNE-induced cytotoxicity, the cytotoxicity of ( $2 R, 4 R)$-AYNE 1, 1-hexadecanol $\left(\mathrm{C}_{16-} \mathrm{OH}\right), 1$-hexadecanoic acid $\left(\mathrm{C}_{16}\right)$, palmitic acid terminal alkyne (PATA), 1-heptadecanol $\left(\mathrm{C}_{17-}\right.$ $\mathrm{OH})$, 1-heptadecanoic acid $\left(\mathrm{C}_{17}\right)$, or heptadecanoic acid terminal alkyne (HATA) were determined on the leukemia cell lines TEX and AML2 (Structures: Figure 6.2.1 A). Out of the lipids tested, the fully saturated fatty alcohols $\left(\mathrm{C}_{16}-\mathrm{OH}, \mathrm{C}_{17}-\mathrm{OH}\right)$ and fatty acids $\left(\mathrm{C}_{16}, \mathrm{C}_{17}\right)$ did not induce $\mathrm{AML}$ death (Grey lines: Figure 6.2.1 B, C). Compared to their fully saturated fatty acid counterparts, the addition of a terminal alkyne induced significant AML death (Red lines: Figure 6.2.1 B, C). Chain length played a critical role in AML cytotoxicity. HATA, a terminal acetylenic 17-carbon long lipid, was significantly more cytotoxic to PATA, a terminal acetylenic 16-carbon long lipid for TEX (Figure 6.2.1 D: $t(4)=32.97, p<0.001)$ and AML2 (Figure 6.2.1 D: $t(4)=19.73, p<0.001)$. Out of all lipids tested, $(2 R, 4 R)$-AYNE 1 was the most toxic compound to TEX (Figure 6.2.1 D: $\mathrm{F}(2,8)=1454, \mathrm{p}<0.001)$ ) and AML2 cells (Figure 6.2.1 D: $\mathrm{F}(2,8)=500, \mathrm{p}<0.001)$. Taken together, having an odd number of carbons in its linear aliphatic chain and a terminal triple bond were critical to AYNE's cytotoxicity. 
A

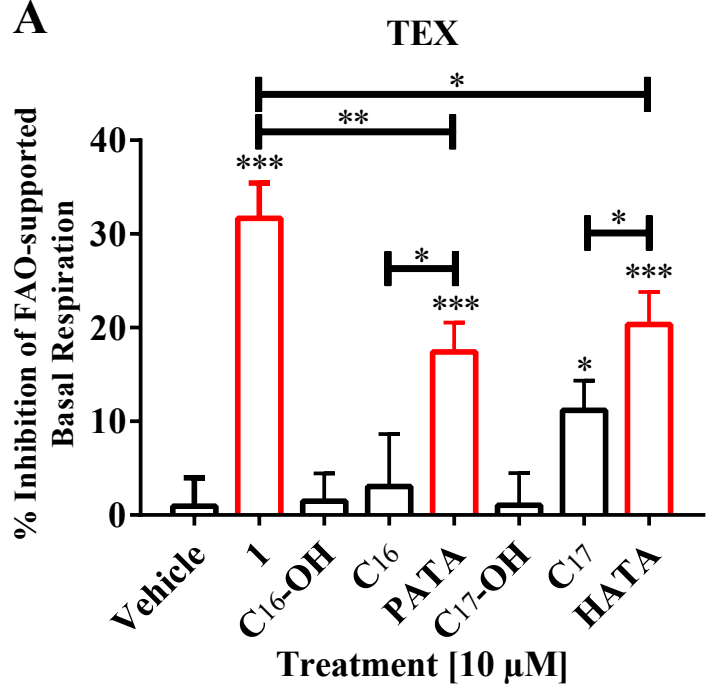

C

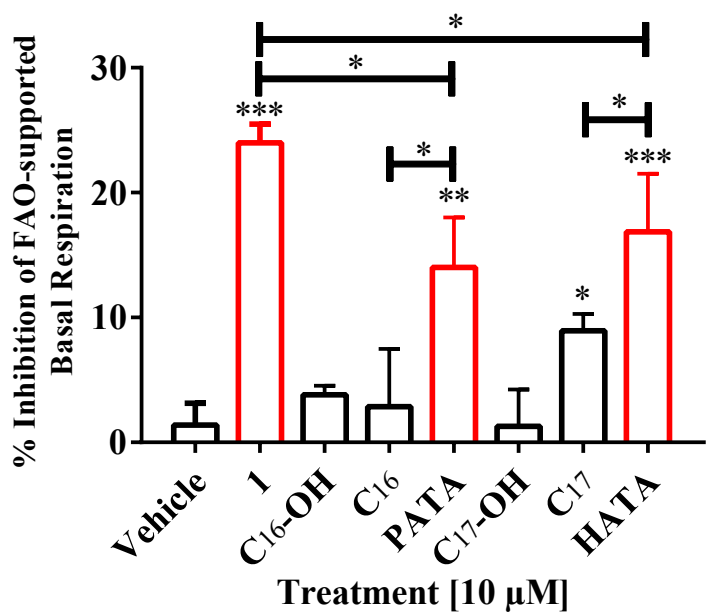

B

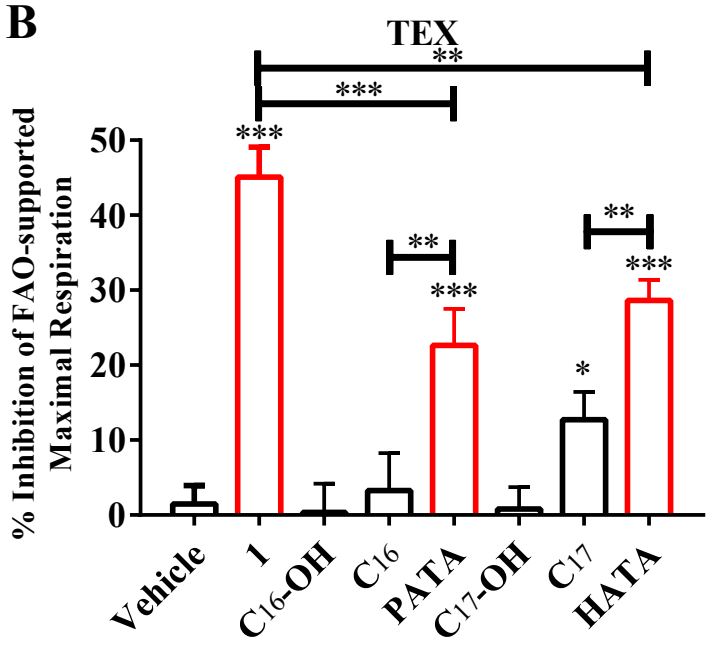

Treatment $[10 \mu \mathrm{M}]$

D

AML2

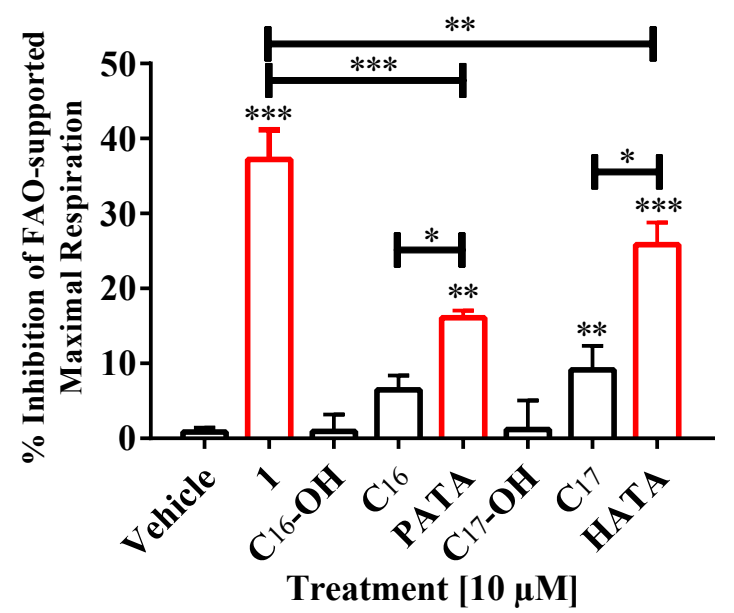

Figure 6.2.2 AYNE's terminal triple bond and an odd number of carbon atoms in its aliphatic linear chain are critical to its ability to suppress mitochondrial respiration.

Assessment of (A) basal and (B) maximal respiration of intact TEX cells following a 1-hour treatment with $10 \mu \mathrm{M}$ of a lipid of interest (N=3). Assessment of (C) basal and (D) maximal respiration of intact AML2 cells following a 1-hour treatment with $10 \mu \mathrm{M}$ of a lipid of interest $(\mathrm{N}=3)$. The bars for AYNE 1, HATA, PATA, the three most potent lipids identified in Figure 6.2.1, are highlighted in red. For (A-D), data is presented as mean \pm S.D., ${ }^{*} p<0.05, * * p<0.01$, $* * * \mathrm{p}<0.001$ using an unpaired, one-tailed, student's t-tests or ANOVA were calculated in GraphPad Prism 7.0. 


\subsubsection{AYNE's Terminal Triple Bond And Odd Numbered Carbon Chain Are Critical To Its Ability To Suppress Mitochondrial Respiration}

Compared to the vehicle control, there was no inhibition of basal or maximal respiration upon treatment with $\mathrm{C}_{16}-\mathrm{OH}, \mathrm{C}_{16}$, and $\mathrm{C}_{17}-\mathrm{OH}$ in intact leukemia TEX (Figure 6.2.2 A, B: Basal: $\mathrm{F}(3,11)=0.19, \mathrm{p}=0.91$; Maximal: $\mathrm{F}(3,1)=0.36, \mathrm{p}=0.79$ ) or AML2 (Figure 6.2.2 C, D: Basal: $\mathrm{F}(3,11)=0.53, \mathrm{p}=0.65 ;$ Maximal: $\mathrm{F}(3,11)=3.77, \mathrm{p}=0.06) . \mathrm{C}_{16}$ is the primary substrate of mitochondria $\mathrm{FAO}$ and while $\mathrm{C}_{17}$ resulted in slight inhibition of mitochondrial respiration in both TEX (Figure 6.2.2 A, B: Basal: $\mathrm{F}(2,8)=5.15, \mathrm{p}=0.05$; Maximal: $\mathrm{F}(2,8)=7.33, \mathrm{p}=0.02)$ and AML2 (Figure 6.2.2 C, D: Basal: $F(2,8)=5.48, p=0.04$; Maximal: $F(2,8)=11.47, p=0.01$ ).

In line with the observed trends in cytotoxicity (Figure 6.2.1), PATA, HATA, and AYNE 1 hindered leukemia cell respiration (Highlighted in red bordered bars in Figure 6.2.2 A-D). The addition of a terminal triple bond to $\mathrm{C}_{16}$ or $\mathrm{C}_{17}$ (PATA or HATA, respectively) increased inhibition in TEX (Figure 6.2.2 A, B: $\mathrm{C}_{16}$ vs PATA Basal: $\mathrm{t}(4)=3.87, \mathrm{p}=0.02$; $\mathrm{C} 16$ vs PATA Maximal: $\mathrm{t}(4)=4.8, \mathrm{p}=0.009 ; \mathrm{C}_{17}$ vs HATA Basal: $\mathrm{t}(4)=3.39, \mathrm{p}=0.03 ; \mathrm{C}_{17}$ vs HATA Maximal: $\mathrm{t}(4) 5.98$,

$\mathrm{p}=0.004$ ) and AML2 (Figure 6.2.2 C, D: $\mathrm{C}_{16}$ vs PATA Basal: $\mathrm{t}(4)=3.16, \mathrm{p}=0.03 ; \mathrm{C}_{16}$ vs PATA Maximal: $\mathrm{t}(4)=7.85, \mathrm{p}<0.001 ; \mathrm{C}_{17}$ vs HATA Basal: $\mathrm{t}(4)=2.84, \mathrm{p}=0.05 ; \mathrm{C}_{17}$ vs HATA Maximal: $\mathrm{t}(4)=6.63, \mathrm{p}=0.003)$.

In contrast to both terminal acetylenic lipids HATA and PATA, AYNE 1 was the most potent inhibitor of intact cell respiration in TEX (Figure 6.2.2 A, B: Basal: $F(2,8)=14.19, p=0.005$; Maximal: $F(2,8)=25.69, p<0.001$ ) and AML2 (Figure 6.2.2 C, D: Basal: $F(2,8)=5.98, p=0.04$; Maximal: $\mathrm{F}(2,8)=39.63, \mathrm{p}<0.001)$. Taken together, the hydroxyl moieties at the $C-2$ and $C-4$ positions, an odd-numbered carbon chain length, and a terminal triple bond are critical to AYNE 1's ability to inhibit fat-supported mitochondrial respiration and induce AML cytotoxicity. 
A
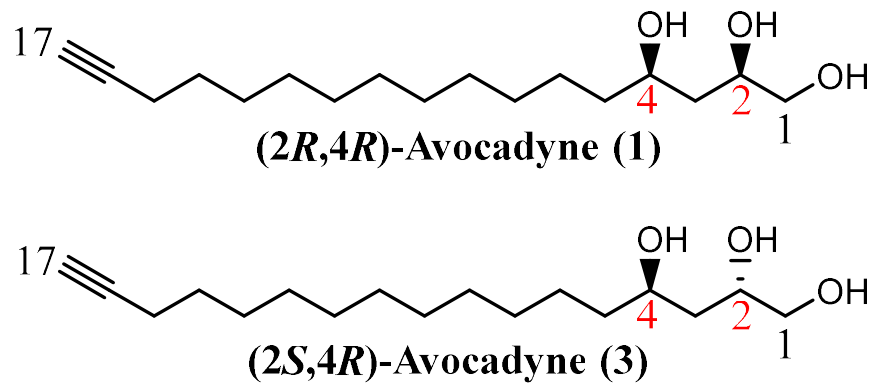

B

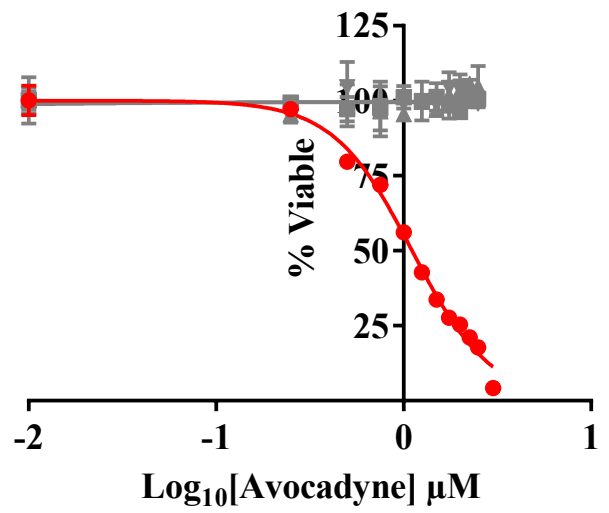

D

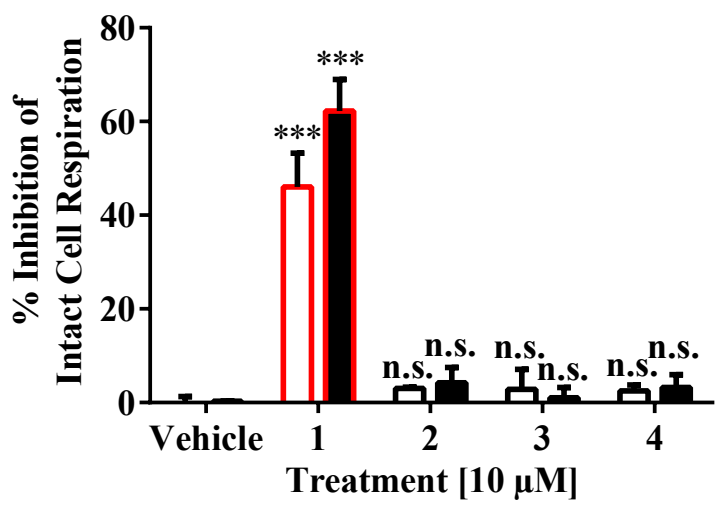

F

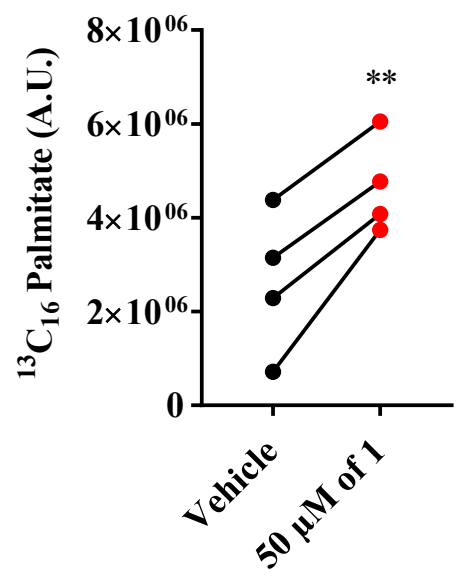

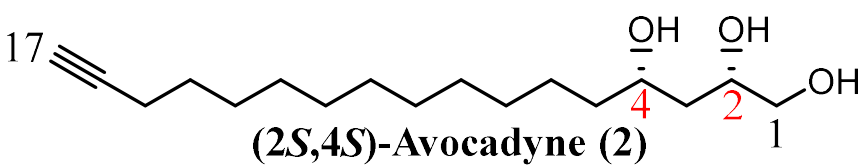

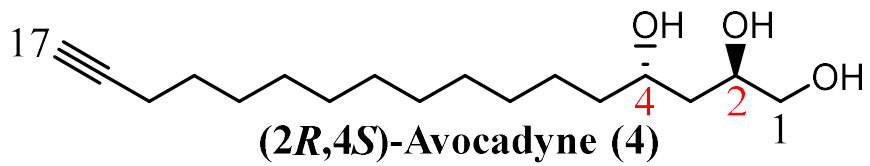

C

AML2

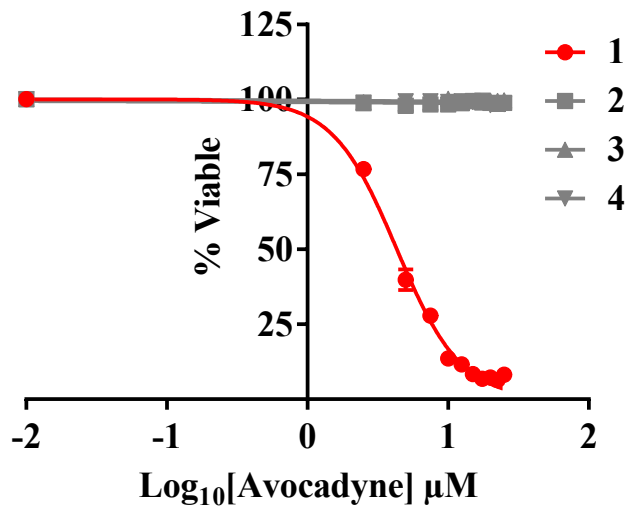

E

AML2

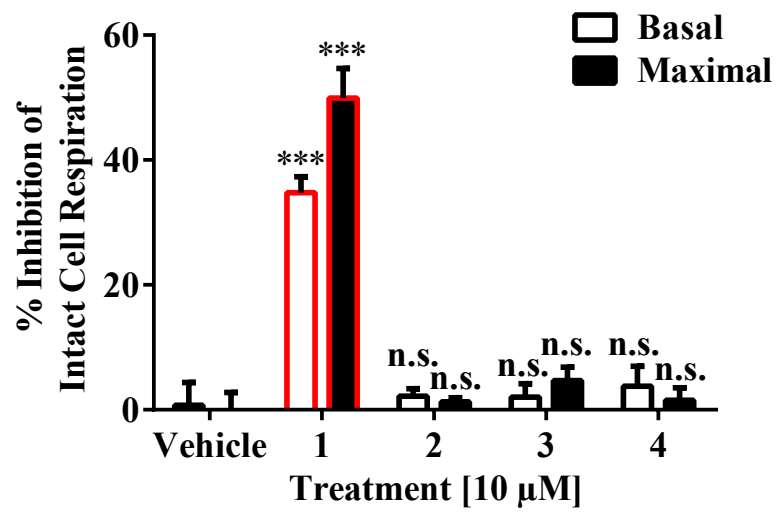

G

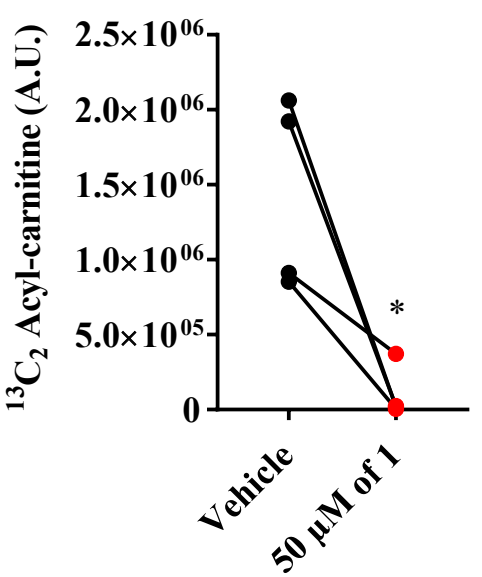


Figure 6.2.3 Stereochemistry at AYNE's chiral carbons are critical to its AML cytotoxicity and ability to inhibit FAO.

(A) Chemical structures of the four synthesized stereoisomers of AYNE 1-4 (Cunha et al., 2019). Chiral carbons $C-2$ and $C-4$ highlighted in red to highlight differences in stereochemistry in compounds 1-4. Structures drawn in ChemDraw 20.0. Cell viability of (B) TEX and (C) AML2 treated with $10 \mu \mathrm{M}$ of an AYNE stereoisomer 1-4 for 72 hours, analyzed with flow cytometry with a 7AAD stain (N=3). Intact cell respiration of (D) TEX and (E) AML2 treated with $10 \mu \mathrm{M}$ of an AYNE stereoisomer 1-4 $(\mathrm{N}=3)$. Assessment of $(\mathbf{F}){ }^{13} \mathrm{C}_{16}$ palmitate and $(\mathbf{G})$ fat-derived ${ }^{13} \mathrm{C}_{2}$ acetylcarnitine as a measure of FAO activity by UHPLC-MS (Jones et al., 2018) in AML patient-derived cells (AML\#1-4) treated with $50 \mu \mathrm{M}$ of the $(2 R, 4 R)$-stereoisomer $\mathbf{1}$ in the presence of a ${ }^{13} \mathrm{C}_{16}$ palmitate tracer for 12 hours. For $(\mathbf{B}-\mathbf{E})$, the bars representing $(2 R, 4 R)$-AYNE 1 are highlighted in red. For (D-G), data is presented as mean \pm S.D., ${ }^{*} p<0.05, * * p<0.01, * * * p<0.001$ using an unpaired, one-tailed, student's $t$-tests or ANOVA were calculated in GraphPad Prism 7.0. For (F, G), MS analysis carried by E. Bohrnsen and Prof. A. D'Alessandro (University of Colorado Anschutz Medical Campus). See patient cytogenetics in Table 6.2.3. 


\subsubsection{Stereochemistry At AYNE's Chiral Carbons Are Critical To Its AML Cytotoxicity And Ability To Inhibit FAO}

The role of stereochemistry at $C-2$ and $C-4$ of four synthetic AYNE stereoisomers (Cunha et al., 2019) (1-4, Structures: Figure 6.2.3 A) was evaluated for anti-AML activity. The activities of the AYNE stereoisomers 1-4 were assessed on AML viability after 72 hours and on intact cell respiration after one hour. In both TEX and AML2, all stereoisomers with a stereocenter of $(S)$ configuration (compounds 2-4) did not induce cell death (Figure 6.2.3 B, C). The $(2 R, 4 R)$ stereoisomer 1 exclusively inhibited intact cell respiration in TEX (Figure 6.2.3 D: Basal: $\mathrm{F}(5,12)=56.13, \mathrm{p}<0.001$; Maximal: $\mathrm{F}(5,12)=111.9, \mathrm{p}<0.001)$ and AML2 (Figure 6.2.3 E: Basal: $\mathrm{F}(5,12)=55.51, \mathrm{p}<0.001 ;$ Maximal: $\mathrm{F}(5,12)=104.0, \mathrm{p}<0.001)$

FAO activity of primary patient-derived AML cells was determined by quantifying levels of uniformly labelled ${ }^{13} \mathrm{C}_{16}$-palmitate, the starting material of the $\mathrm{FAO}$ pathway and the primary substrate of the VLCAD enzyme, and the fat-derived ${ }^{13} \mathrm{C}_{2}$ acetyl-carnitine metabolite, the final product of the FAO pathway, by UHPLC coupled to high resolution MS (Jones et al., 2018). Treatment with the active $(2 R, 4 R)$-stereoisomer 1 confirmed FAO inhibition of primary AML cells, as determined by an accumulation of palmitate (Figure 6.2.3 F: $\mathrm{t}(3)=6.048, \mathrm{p}=0.009$ ) and a decrease in acetyl-carnitine (Figure 6.2.3 G: $\mathrm{t}(3)=3.549, \mathrm{p}=0.04)$. Taken together, $(2 R, 4 R)$ stereochemistry is critical to AYNE's ability to inhibit FAO in both immortalized cell lines and primary AML samples. 
A<smiles></smiles><smiles>CC(O)[C@H](O)C[C@@H](O)CCCCCCCCCCCCCCCCCC=[In]</smiles><smiles>CC(O)C(O)C[C@H](O)CCCCCCCCCCCCCCCCCC=[In]</smiles><smiles>C=CCCCCCCCCCCCC[C@H](O)C[C@@H](O)C(C)O</smiles>

B

TEX

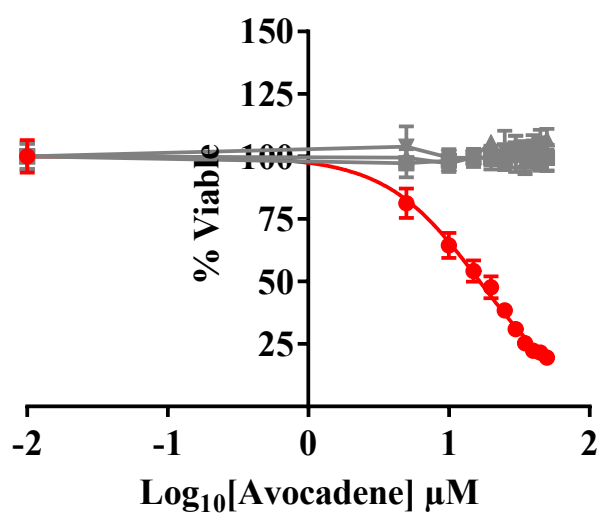

D

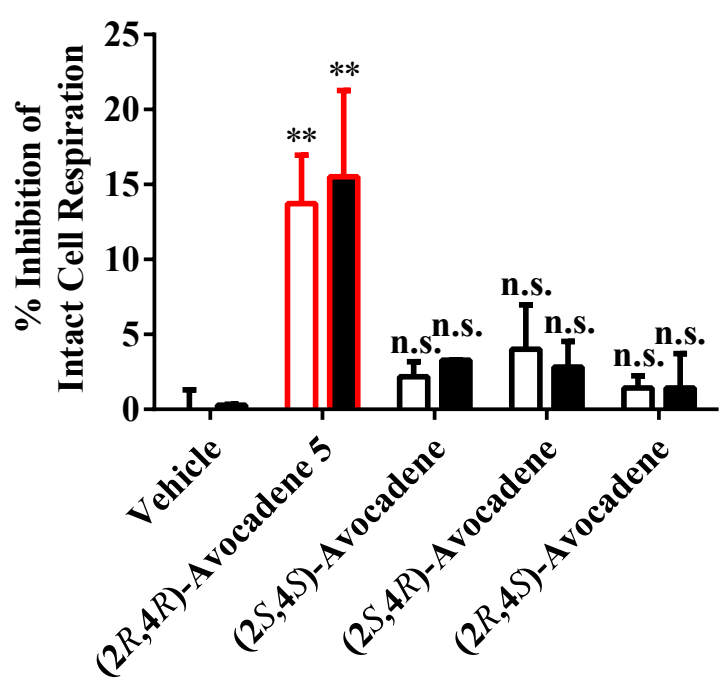

Treatment [10 $\mu \mathrm{M}]$
C

AML2

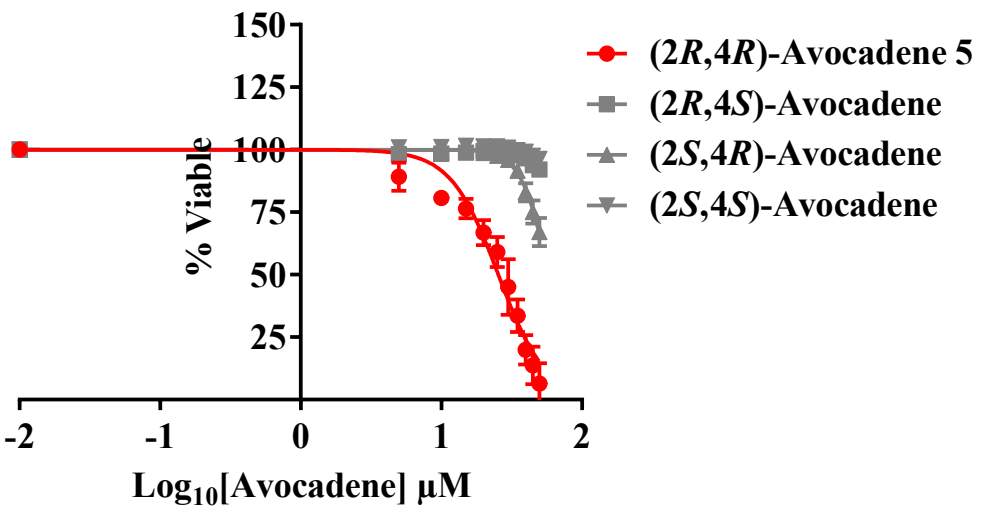

E

AML2

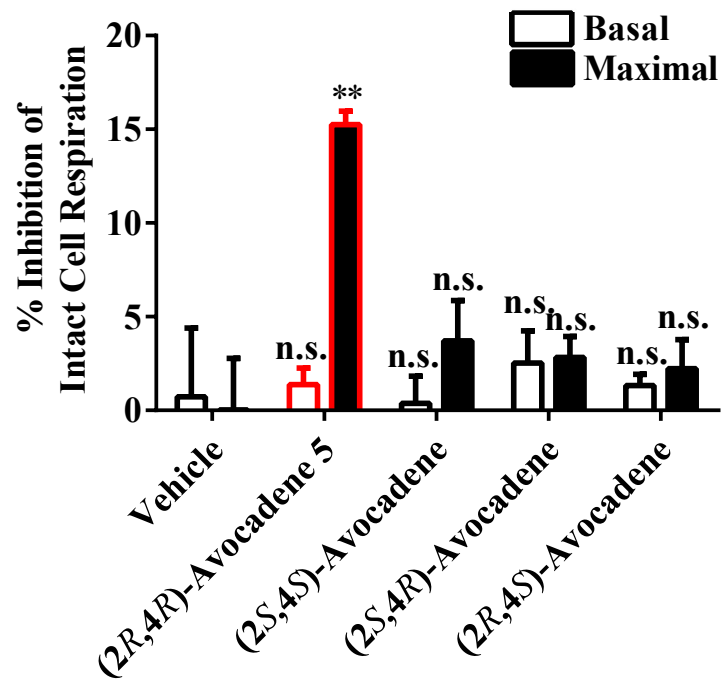

Treatment [10 $\mu \mathrm{M}]$ 
Figure 6.2.4 Stereochemistry at avocadene's chiral carbons are critical to AML cytotoxicity and ability to inhibit mitochondrial respiration.

(A) Chemical structures of the four stereoisomers of avocadene (Cunha et al., 2019). Chiral carbons $C-2$ and $C-4$ highlighted with red numbers to underline differences in stereochemistry in AENE stereoisomers. Structures drawn in ChemDraw 20.0. Cell viability of (B) TEX and (C) AML2 treated with $10 \mu \mathrm{M}$ of an avocadene stereoisomer for 72 hours, analyzed with flow cytometry with a 7AAD stain $(\mathrm{N}=3)$. Assessment of intact cell respiration of (D) TEX and (E) AML2 treated with $10 \mu \mathrm{M}$ of an avocadene stereoisomer 1-4 $(\mathrm{N}=3)$. For (B-E), activity of the $(2 R, 4 R)$-AENE $\mathbf{5}$ is highlighted in red. For $(\mathbf{B}, \mathbf{C}), \mathrm{IC}_{50}$ values were calculated using dose response - inhibition equation on GraphPad Prism 7.0. For (D, E), data is presented as mean \pm S.D., ${ }^{*} \mathrm{p}<0.05,{ }^{*} \mathrm{p}<0.01,{ }^{* * *} \mathrm{p}<0.001 \mathrm{using}$ an unpaired, one-tailed, student's $t$-tests or ANOVA were calculated in GraphPad Prism 7.0. 


\subsubsection{Stereochemistry At Avocadene's Chiral Carbons Are Critical To AML Cytotoxicity And Ability To Inhibit Mitochondrial Respiration}

The role of stereochemistry at $C-2$ and $C-4$ of four synthetic AENE stereoisomers (Structures: Figure 6.2.4 A) was evaluated for anti-AML activity. The activities of the AENE stereoisomers were assessed on AML viability after 72 hours and on intact cell respiration after one hour. In both TEX and AML2, all stereoisomers with $(S)$-configuration at either $C$-2 or $C$-4 did not induce cell death (Grey lines in Figure 6.2.4 B, C). The active $(2 R, 4 R)$ stereoisomer 5 exhibited $\mathrm{IC}_{50}$ values of $12.72 \pm 0.64 \mu \mathrm{M}$ on TEX and $28.57 \pm 2.16 \mu \mathrm{M}$ on AML2 (Red lines in Figure 6.2.4 B, C). The $(2 R, 4 R)$-stereoisomer of AENE 5 exclusively inhibited intact basal and maximal respiration in TEX (Red-bordered bars in Figure 6.2.3 D: Basal: $F(5,12)=41.25, p=0.02$; Maximal: $F(5,12)=72.51, p=0.01$ ) and only maximal respiration in AML2 (Red-bordered bars in Figure 6.2.3 E: Basal: $F(5,12)=21.29, p=0.072$; Maximal: $F(5,12)=82.1, p=0.02)$. These results confirmed that, similarly to $(2 R, 4 R)$-AYNE $\mathbf{1}$, stereochemistry is critical to $(2 R, 4 R)$-AENE 5's ability to induce death in AML cells and inhibit fat-supported respiration. 


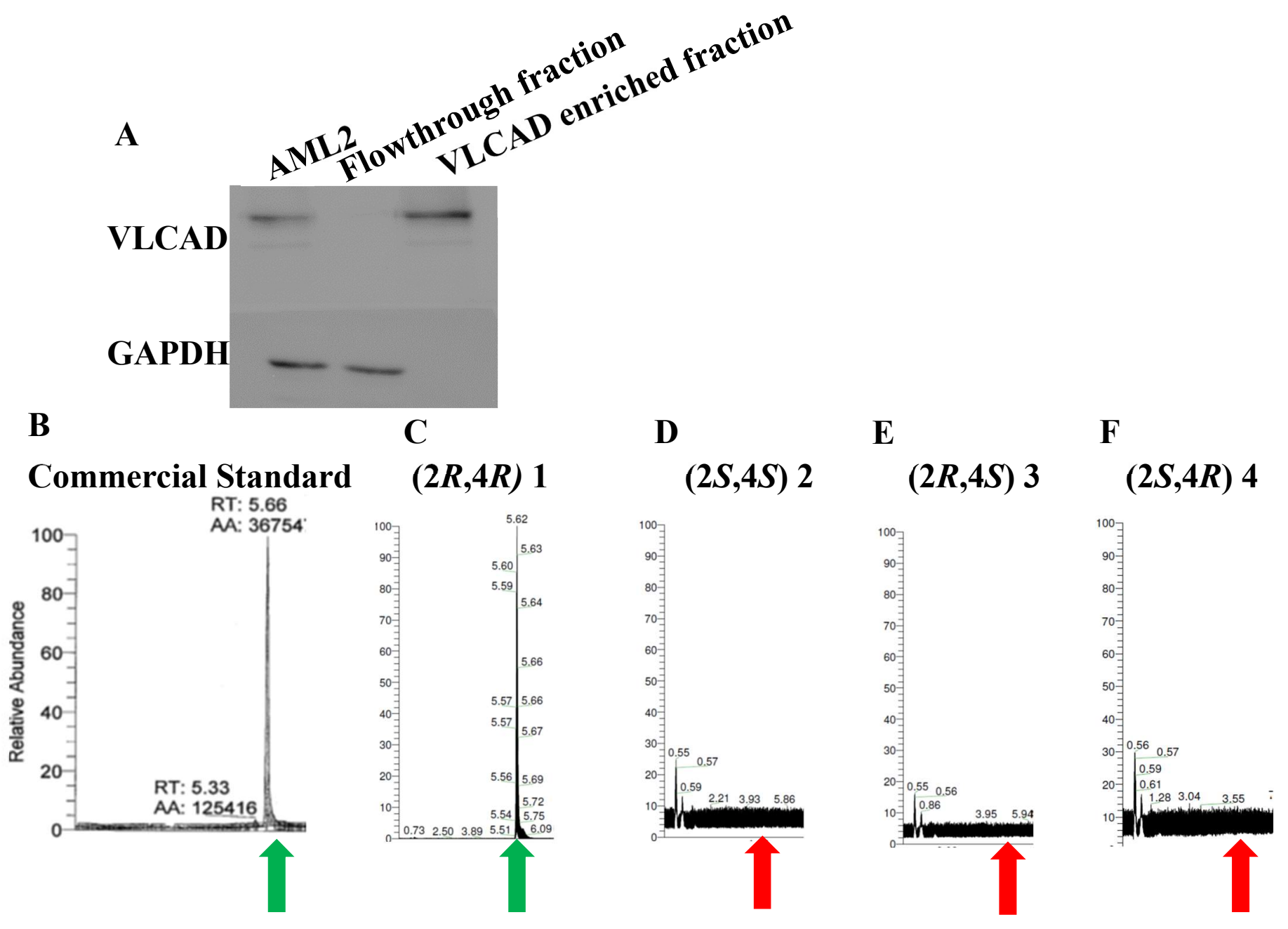

Figure 6.2.5 (R)-stereochemistry is critical for AYNE to directly interact with VLCAD. AML2 cells were treated with $10 \mu \mathrm{M}$ of an AYNE stereoisomer 1-4 for three hours and coimmunoprecipitated against an anti-VLCAD antibody ( $\mathrm{N}=3$ per stereoisomer). (A) Immunoblot demonstrating purity of the VLCAD enriched fraction. (B) Chromatogram of a commercial standard of AYNE extracted from avocadoes. Chromatograms of VLCAD-enriched fractions treated with (C) the $(2 R, 4 R)-\mathbf{1}$, (D) $(2 S, 4 S)-\mathbf{2}$, (E) $(2 R, 4 S)-\mathbf{3}$, (F) $(2 S, 4 R)$-stereoisomers 4 of AYNE ( $\mathrm{N}=3$ per stereoisomer). MS analysis was carried out using a previously published analytical method (Ahmed et al., 2018); quantifications are tabulated in Table 6.2.2. MS analysis carried by N. Ahmed (University of Guelph Food Science) and Prof. R. W. Smith (University of Waterloo Chemistry).

Table 6.2.2 AYNE recovered from AML cells treated with compounds 1-4 by co-IP

\begin{tabular}{|c|c|}
\hline Compound \# & Concentration recovered (nM) \\
\hline $\mathbf{1}$ & $209.13 \pm 48.67$ \\
\hline $\mathbf{2}$ & $<\mathrm{LOD}$ \\
\hline $\mathbf{3}$ & $<\mathrm{LOD}$ \\
\hline $\mathbf{4}$ & $<\mathrm{LOD}$ \\
\hline
\end{tabular}




\subsection{5 (R)-Stereochemistry Is Critical For AYNE To Directly Interact With VLCAD}

Previously in chapter 5, AYNE inhibited FAO by interacting directly with VLCAD, as confirmed by co-IP (Figure 5.2.9). To assess the role of stereochemistry in VLCAD binding, AML2 cells were co-incubated with $10 \mu \mathrm{M}$ of one of the four stereoisomers 1-4 for three hours, lysed, and then subjected to co-immunoprecipitation with an anti-VLCAD antibody. VLCADenriched fractions were then tested for purity by immunoblotting (Figure 6.2.5 A) or subjected to LC/MS/MS analysis for AYNE quantitation (Ahmed et al., 2018). The commercial standard of extracted AYNE elutes at 5.6 minutes (Figure 6.2.5 B). Consistent with the respiration and cytotoxicity data (Figure 6.2.1-6.2.2), only the $(2 R, 4 R)$-stereoisomer 1 was detected above the quantification limit in VLCAD-enriched fractions with a peak eluting at 5.6 minutes, suggesting that only this stereoisomer interacts to VLCAD (Figure 6.2.5 C, Table 6.2.2). The chromatograms for the 3 other stereoisomers did not show a peak at 5.6 minutes (Figure 6.2.5 D-F, Table 6.2.2), suggesting that these three stereoisomers do not interact in VLCAD. Taken together, $(2 R, 4 R)$ stereochemistry is critical to AYNE binding to VLCAD. 
Treat with vehicle

\section{A}

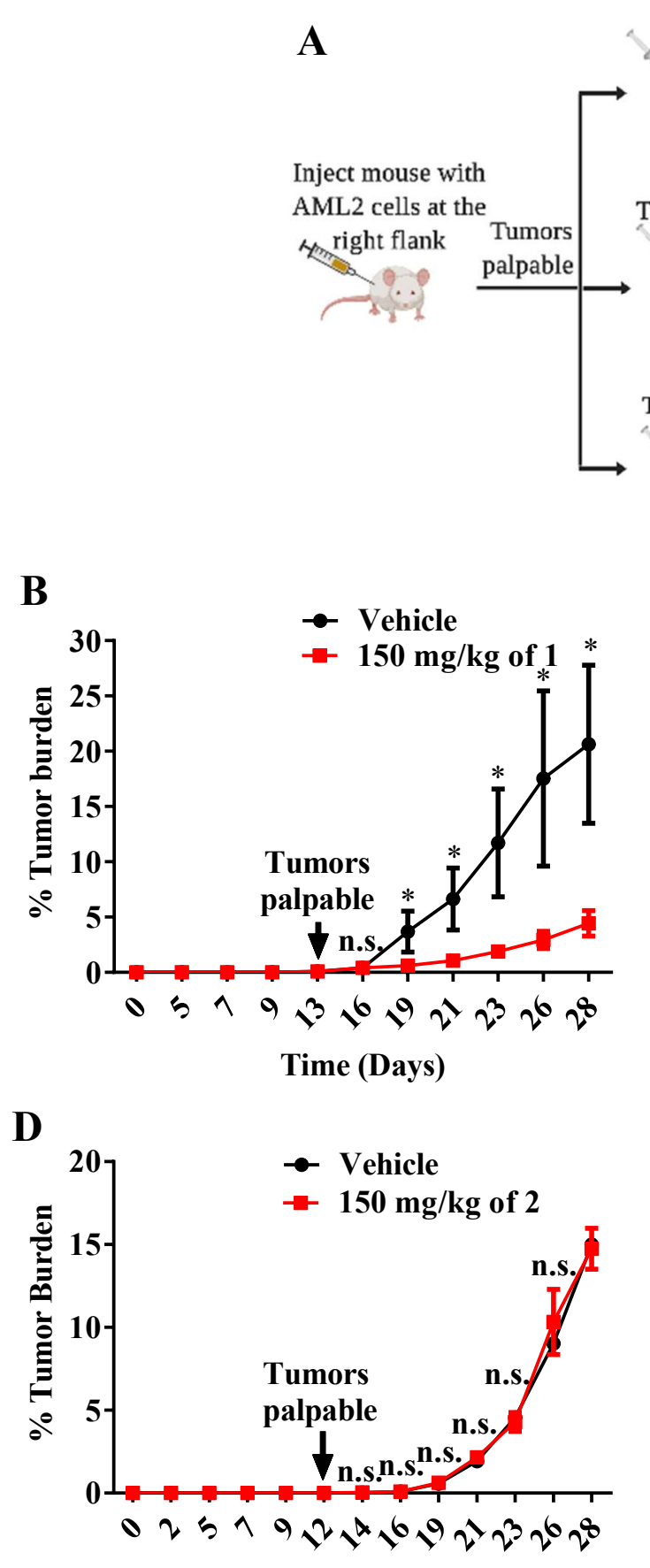

Time (Days)
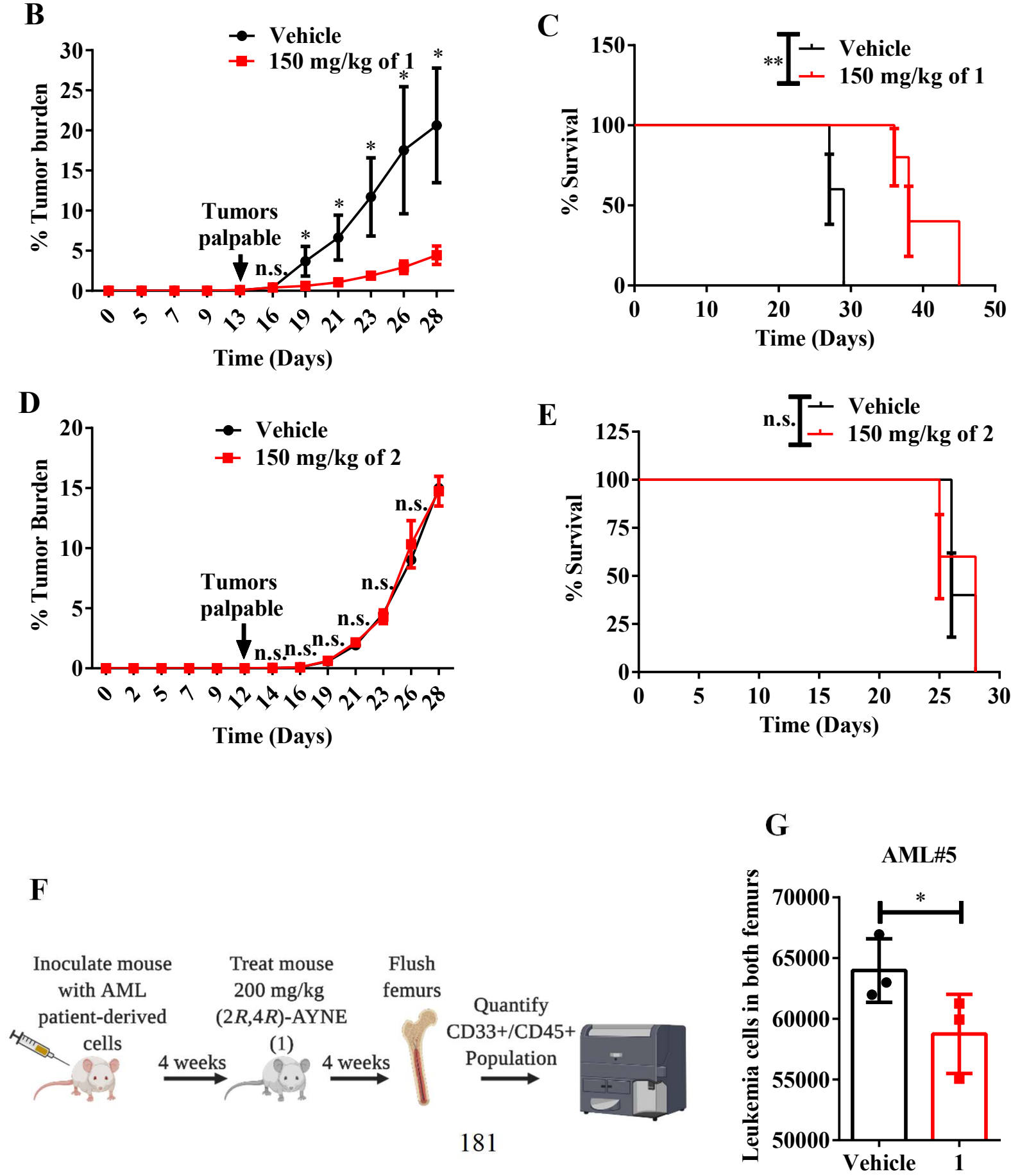
Figure 6.2.6 $(2 R, 4 R)$-stereochemistry is critical for AYNE's in vivo anti-AML activity. (A) Schematic showing AML2 cells being injected into the right flanks of NSG mice. Once tumors were palpable, mice were divided into three groups and treated with either a vehicle emulsion or an emulsion containing either $150 \mathrm{mg} / \mathrm{kg} /$ week of the $(2 R, 4 R)-\mathbf{1}$ or the $(2 S, 4 S)$-stereoisomers 2 of AYNE ( $\mathrm{N}=5$ per group). Created in Biorender. (B) Tumor formation and (C) survival of tumor bearing mice treated with the $(2 R, 4 R)$-stereoisomer of AYNE 1. (D) Tumor formation and (E) survival of tumor bearing mice treated with the $(2 S, 4 S)$-stereoisomer of AYNE 2. (F) Schematic showing, after tail vein injection, human AML patient cells (AML\#5) were given four weeks to engraft in mouse femurs. Mice then received $200 \mathrm{mg} / \mathrm{kg} /$ week $(2 R, 4 R)$-stereoisomer of AYNE 1 for four weeks. Created in Biorender. (G) Engraftment levels (\%CD33+/CD45+) from AML\#5 were quantified by flow cytometry. See Table 6.2.3 for patient cytogenetics. For $(\mathbf{B}, \mathbf{D}, \mathbf{G})$, $* \mathrm{p}<0.05, * * \mathrm{p}<0.01, * * * \mathrm{p}<0.001$ using unpaired, two-tailed, Mann Whitney's t-tests were calculated in GraphPad Prism 7.0. For $(\mathbf{C}, \mathbf{E})$, differences in survival were calculated with the Mantel-Cox Log-Rank test in GraphPad Prism 7.0. In vivo studies carried out by A. Roma and P. Jayanth. 


\subsection{6 (2R,4R)-Stereochemistry Is Critical For AYNE's In Vivo Anti-AML Activity}

Next, the role of stereochemistry at $C-2$ and $C-4$ in AYNE's anti-AML activity in vivo was determined. AML2 cells were xeno-transplanted into the right flanks of NSG mice (Study schematic in Figure 6.2.6 A). Once tumors were palpable, mice were treated with a vehicle emulsion (Ahmed et al., 2020) or $150 \mathrm{mg} / \mathrm{kg}$ of either the $(2 R, 4 R)$-stereoisomer 1 or $(2 S, 4 S)$ stereoisomer $\mathbf{2}$ every other day for fourteen days until endpoint. Only the $(2 R, 4 R)$-stereoisomer $\mathbf{1}$ suppressed tumor formation survival (Figure 6.2.6 B: Day $13 \mathrm{t}(4)=1.881, \mathrm{p}=0.1331$; Day 16 $\mathrm{t}(6)=0.1175, \mathrm{p}=0.1678 ;$ Day $19 \mathrm{t}(6)=1.833, \mathrm{p}=0.1165 ;$ Day $21 \mathrm{t}(7)=2.810, \mathrm{p}=0.0262 ;$ Day 23 $\mathrm{t}(7)=2.852, \mathrm{p}=0.0246$; Day $26 \mathrm{t}(7)=2.521, \mathrm{p}=0.0398$; Day $28 \mathrm{t}(7)=3.704, \mathrm{p}=0.0076)$, resulting in extended murine (Figure 6.2.6 C: Log Rank Test $\mathrm{p}=0.0031)$. In contrast, the $(2 S, 4 S)$-stereoisomer 2 did not suppress tumor formation (Figure 6.2.6 D: Day $14 \mathrm{t}(8)=0.6905, \mathrm{p}=0.5094$; Day 16 $\mathrm{t}(8)=0.06186, \mathrm{p}=0.4761 ;$ Day $19 \mathrm{t}(8)=0.3324, \mathrm{p}=0.3741 ;$ Day $21 \mathrm{t}(8)=0.5581, \mathrm{p}=0.2960 ;$ Day 23 $\mathrm{t}(8)=0.3576, \mathrm{p}=0.3649 ;$ Day $26 \mathrm{t}(8)=0.6192, \mathrm{p}=0.2765$; Day $28 \mathrm{t}(3)=0.09170, \mathrm{p}=0.89)$ and showed no difference in survival, compared to the vehicle group (Figure 6.2.6 E: Log Rank Test $\mathrm{p}=0.8978)$

To confirm the inhibitory activity of the $(2 R, 4 R)$-stereoisomer 1 on leukemia engraftment, a NSG engraftment assay was employed. Primary patient AML cells were injected via tail vein and allowed four weeks to engraft to the femoral bone marrow (Study schematic in Figure 6.2.6 F). Following engraftment, mice receiving $200 \mathrm{mg} / \mathrm{kg} /$ weekly of the $(2 R, 4 R)$-stereoisomer 1 for four weeks demonstrated reduced leukemia engraftment, compared to the vehicle control (Figure 6.2.6 G: $\mathrm{p}=0.05)$. This highlights the in vivo activity of $(2 R, 4 R)$-stereoisomer 1 , confirming that stereochemistry plays a critical role in AYNE's mechanism of action. 
Table 6.2.3 Patient IDs for Chapter 6.

\begin{tabular}{|c|c|}
\hline Patient ID & Cytogenetics \\
\hline AML\#1 & $46, \mathrm{XX}[20]$ \\
\hline AML\#2 & $46, \mathrm{XX}, \mathrm{t}(9 ; 22)(\mathrm{q} 34 ; \mathrm{q} 11.2)[20]$ \\
\hline AML\#3 & $46, \mathrm{XX}[20]$ \\
\hline AML\#4 & $47, \mathrm{XX},+8, \operatorname{add}(22)(\mathrm{q} 13)[10]$ \\
\hline AML\#5 & $48, \mathrm{XX},+8,+20[16] / 46, \mathrm{XX}[4]$ \\
\hline
\end{tabular}




\subsection{Discussion}

These results in this chapter investigated the hypotheses set out in chapter 2 to determine the structural moieties critical to avocadyne's ability to suppress mitochondrial metabolism. In chapter 6 , an odd number of carbons in the aliphatic linear chain, hydroxyl groups with $(R)$ stereochemistry at $C-2$ and $C-4$, and a terminal triple bond were shown to be critical structural elements in AYNE required for FAO inhibition at VLCAD and for AML cytotoxicity.

FAO of lipids with an odd number of carbon atoms is distinct from the oxidation of lipids with an even number of carbon atoms due to the recruitment of additional enzymes (Gotoh et al., 2008)(Shibata et al., 2012). In Figure 6.2.2, the fully saturated heptadecanoic acid $\left(\mathrm{C}_{17}\right)$ reduced FAO-supported mitochondrial respiration, demonstrating that the oxidation of lipids with an odd number of carbons is less efficient, compared to even numbered counterparts. 24 hours after mice were infused with ${ }^{13} \mathrm{C}$-labelled fatty acids that were $15,16,17,18$ carbon atoms long, the fats with an odd number of carbon atoms accumulated, suggesting slower FAO activity (Gotoh et al., 2008)(Shibata et al., 2012). Since FAO shortens fatty acids by two carbon atoms per cycle, the oxidation of a fat with an odd number of carbon atoms will result in the production of propionylCoA, a three-carbon metabolite. To avoid propionic acidosis and metabolic dysfunction from the oxidation of $\mathrm{C}_{15}$ and $\mathrm{C}_{17}$ fatty acids, the enzymes propionyl-CoA carboxylase, methylmalonylCoA mutase, and methylmalonyl-CoA epimerase must be recruited to convert propionyl-CoA to succinyl-CoA, a metabolite that can be utilized in the TCA cycle. In contrast, the oxidation of fats with an even number of carbons produce the two carbon long acetyl-CoA, ready for TCA metabolism without the recruitment of additional enzymes (Houten \& Wanders, 2010). These observations presented in chapter 6 are in agreement with previous studies demonstrating that long chain fats with an even number of carbon atoms, in contrast to lipids with an odd number of carbon 
atoms, are more efficiently processed by mitochondrial FAO (Gotoh et al., 2008)(Shibata et al., 2012).

Terminal triple bonds are critical moieties in terminally acetylenic fatty acids, a class of synthetic suicide inhibitors of the cytochrome P450 enzyme family (Muerhoffs et al., 1989)(Yap et al., 2010). Cytochrome P450 isoforms catalyze $\omega$-oxidation of fats in kidney nephrotic cells, hepatocytes (Muerhoffs et al., 1989), and leukocytes (Yap et al., 2010). 15-hexadecynoic and 17octadecynoic acids, both terminal acetylenic fatty acids of similar length to AYNE, were not cytotoxic to nephrotic cells, hepatocytes, or leukocytes at anti-AML concentrations and are being established at modulators of immune response (Muerhoffs et al., 1989)(Yap et al., 2010). In line with these previous studies, no change in viability was observed with normal blood cells and no changes in blood cell count as well as kidney and liver function were noted in in vivo mice studies with anti-AML AYNE doses presented in chapter 5 and 6.

Despite the structural similarities to previously studied acetylenic fatty acids (Muerhoffs et al., 1989)(Yap et al., 2010), a previous study (Lee et al., 2015) as well as chapters 4 and 5 of this thesis confirm that AYNE's primary bioactivity in AML cells is FAO inhibition. Previously, subcellular fractionation experiments determined that the constituents of avo-B accumulated in the mitochondria (Lee et al., 2015). In addition, compared to parental Jurkat cells, Jurkat $\rho 0$ cells, a cell line with depleted mitochondria mass, were insensitive to avo-B, suggesting the mitochondria is critical to AML cytotoxicity by avo-B (Lee et al., 2015). In chapter 5 of the current thesis, AYNE co-immunoprecipitated with VLCAD, an intramitochondrial FAO enzyme. Supplementation with heptanoate, a medium chain fatty acid, also rescued AML cells from inhibition of mitochondrial FAO. Taken together, these experiments confirm that the primary metabolic target of AYNE is mitochondrial FAO, which is distinct from the previously studied acetylenic fatty acids which 
target $\omega$-oxidation, a metabolic process localized to the endoplasmic reticulum (Muerhoffs et al., 1989)(Yap et al., 2010). Future experiments are required to delineate the bioactivities of terminal acetylenic fatty acids and terminal acetylenic fatty alcohols to confirm that inhibition of cytochrome P450 is not a significant off target of AYNE in AML.

In agreement with previous studies (Oakes et al., 1999)(Makowski et al., 2013)(Agius et al., 1991), chirality is critical when designing compounds targeting mitochondrial FAO. In this chapter, deviation from $(R)$-stereochemistry at either $C-2$ and $C-4$ resulted in complete attenuation of VLCAD binding, FAO inhibition, and AML cytotoxicity by AYNE. Similarly, any stereoisomer of AENE deviating from $(R)$-stereochemistry at either $C-2$ or $C-4$ was not cytotoxic to AML. Having $(R)$-configuration at $C$-2 in FAO inhibitors such as 2-bromopalmitate (Oakes et al., 1999), C-75 (Makowski et al., 2013), and the entire class of CPT1-targeting oxiranes (which includes etomoxir) was critical for FAO inhibition. FAO inhibition is observed with the $(R)$-enantiomer while fatty acid synthesis inhibition is observed with the $(S)$-enantiomer of the aforementioned compounds (Agius et al., 1991).

While previous studies (Lee et al., 2015)(Ahmed et al., 2020)(Tabe et al., 2018) utilized the $(2 R, 4 R)$-AYNE stereoisomer extracted from avocadoes, the three remaining possible configurations have not been elucidated from the fruit. The levels of extracted acetogenins, such as AYNE, fluctuates based on avocado maturation stage, climate, soil composition, fertilizer use, tissue (peel, pulp, or seed), and by cultivar (Salazar-López et al., 2020)(Rodríguez-López et al., 2017). The results presented in this chapter confirmed that the terminal triple bond, and the stereochemistry of the hydroxyl groups, and an odd number of carbon atoms in its aliphatic linear chain were all critical to AYNE bioactivity in AML. Future studies demonstrating anti-AML 
efficacy with AYNE should utilize exclusively the synthesized $(2 R, 4 R)$-stereoisomer to avoid differences in extracted AYNE yield due to cultivation conditions. 


\subsection{Chapter 7 Discussion, Limitations, And Conclusion}

\subsection{Discussion}

AML is a devastating blood cancer with limited therapeutic options that fail to eliminate the entire cancer cell population and are toxic to the normal hematopoietic population (Löwenberg et al., 2011)(Khwaja et al., 2016). Compared to the normal hematopoietic population, AML populations exhibit a distinct mitochondrial phenotype characterized by an increased reliance on higher rates of FAO (Samudio et al., 2010)(German et al., 2016) with a diminished capacity to oxidize other substrates upon inhibition of oxidative metabolism (Molina et al., 2018)(Lagadinou et al., 2013)(Sharon et al., 2019). Targeting this novel metabolic vulnerability through the inhibition of FAO at VLCAD, a previously unexplored metabolic enzyme in AML, induced death of leukemia cells, while sparing normal hematopoietic cells, highlighting the pre-clinical potential of a novel therapeutic avenue in AML. In this thesis, the mechanism of action and the critical structural components of avocadyne, the anti-AML component of the avocado-derived mixture avo-B, were elucidated.

Previously, the leukemia residual cells surviving either cytarabine (Farge et al., 2017) or the venetoclax/azacytidine (Stevens et al., 2020) regimen exhibited increased mitochondrial oxidative metabolism fueled by long chain FAO to survive and initiate AML re-population; these studies highlighted the need for novel selective inhibitors of mitochondrial respiration to suppress in the increased mitochondrial metabolism resulting from current chemotherapeutics. In chapter 4, the mitochondria targeting avo-B mixture synergized with induction chemotherapeutics ara-C and DOX to induce AML death, in agreement with previous studies demonstrating that FAO inhibitors restored AML sensitivity to induction chemotherapeutics by depleting ATP levels (Farge et al., 2017)(Stevens et al., 2020). In line with these previous studies using the FAO inhibitors ETO and 
SSO, the avo-B mixture previously sensitized the AML3 cell line to ara-C induced death by triggering an energy crisis. This resulted in the activation of the AMP-activated protein kinase (AMPK), a protein that stimulates energy producing pathways, such as glycolysis, and suppresses pathways support cell proliferation, such as the mammalian target of rapamycin (mTOR) pathway, upon metabolic or pharmacological stress (Tabe et al., 2018). The results presented in chapters 4 and 5 are in agreement with these previous studies (Farge et al., 2017)(Stevens et al., 2020) demonstrating that FAO inhibitors trigger an energy crisis to sensitize AML to chemotherapy and establish that AYNE, the active agent of avo-B, is responsible for ATP depletion.

Chapter 5 elucidated the role of VLCAD, an overexpressed intramitochondrial FAO enzyme that was previously unexplored in AML mitochondrial metabolism. Previously, avo-B accumulated within the mitochondria to hinder mitochondrial metabolism, suggesting a novel intramitochondrial FAO target (Lee et al., 2015). Anchored to the IMM facing the mitochondrial matrix and in close proximity to the ETC, the VLCAD enzyme catalyzes the first intramitochondrial FAO reaction for long chain fats (McAndrew et al., 2008). The oxidation of the fat results in the reduction of the ETF, which passes electrons into the ETC bypassing CI and CII. Loss of VLCAD in humans hindered mitochondrial oxidative metabolism by depleting TCA metabolites and ATP in tissues reliant on FAO, such as the heart, liver, and skeletal muscle. These non-transformed FAO-dependent tissues robustly switched to glycolysis to meet energy needs, resulting in hypoglycemia in some patients of VLCAD deficiency (Vockley et al., 2016). In contrast, the results presented in chapter 5 demonstrated that a consequence of metabolic rewiring to support uncontrolled AML proliferation was the lack of capacity to upregulate alternative metabolic pathways, such as glycolysis; this distinct metabolic vulnerability confirmed that AML cells were less able to adapt to changes in substrate availability, further suggesting a therapeutic 
window for targeting mitochondrial metabolism in AML. Adult human patients of VLCAD deficiency manage symptoms by avoiding dietary long chain fats, cold exposure, prolonged fasting, and strenuous physical activity, suggesting that VLCAD inhibition in AML is feasible (Schiff et al., 2013).

Chapter 5 demonstrated divergent metabolic fates upon VLCAD inhibition with extensive metabolic assessment with stable isotope tracing experiments of both the malignant AML and normal hematopoietic populations, further underscoring a potential therapeutic window. Selective AML cell death via VLCAD inhibition was due to the overreliance on higher rates of FAO (Samudio et al., 2010)(German et al., 2016) and the lack of a capacity to switch to alternative substrates to support energy metabolism (Molina et al., 2018)(Lagadinou et al., 2013)(Sharon et al., 2019). Previously, AML populations capable of upregulating glycolysis-linked lactate production overcome inhibition of mitochondrial oxidative metabolism, due to NAD+ regeneration (Lunt \& Vander Heiden, 2011) and the kinetically favorable production of ATP (Jose et al., 2011), compared to OXPHOS.

Pharmacological activation with the ISRIB artificially increased glycolysis-linked lactate production to rescue malignant cells. Preceding cell death, AML3 cells treated with avo-B upregulated the activating transcription factor 4 (ATF4), a transcription factor that is induced by the integrated stress response (ISR), a signalling pathway that modulates apoptosis in response to mitochondrial stress (Tabe et al., 2018). The ISR/ATF4 signalling axis modulates cell fate. Prolonged ISR signalling suppressed lactate production, resulting in AML death; inhibition of ISR by the pharmacological activator ISRIB increased compensatory glycolysis, resulting in AML survival (Sharon et al., 2019). While not assessed in this thesis, the modulating of compensatory glycolysis by the ISR/ATR4 signalling pathway in AML cells underlined the activation of 
glycolysis-lactate production that was previously shown to support ATP production upon inhibition of mitochondrial metabolism by avo-B (Tabe et al., 2018) and venatoclax (Sharon et al., 2019). In agreement with this, AYNE triggered an energy crisis and AML death, without sufficient compensatory metabolism, while ATP levels were not altered in normal MNC, which enabled cell survival. In contrast, AML cell lines and primary samples that were sensitive to FAO inhibition at VLCAD demonstrated increased PDH activity, previously shown to hinder AML proliferation and engraftment capacity (Wang et al., 2014)(Hao et al., 2019). This difference in the metabolic reliance on FAO activity and the capacity to upregulate compensatory metabolism distinguished the AML and normal hematopoietic populations, further demonstrating a therapeutic window for targeting FAO at VLCAD. Taken together, the results presented in chapter 5 are in agreement with previous studies (Samudio et al., 2010)(German et al., 2016)(Shafat et al., 2017)(Zhang et al., 2020)(Wu et al., 2015), confirming that AML sensitivity to FAO blockade is due to metabolic rigidity. Pharmacodynamic assessment of avocadyne also establishes the pre-clinical potential of chemical inhibition of VLCAD as a novel anti-AML target critical to mitochondrial metabolism.

In chapter 6, a structure-activity relationship identified the critical structural components responsible for AYNE's mechanism of action. In agreement with previous studies, an odd number of carbons in the aliphatic linear chain (Gotoh et al., 2008)(Shibata et al., 2012), a terminal triple bond, and (2R,4R)-stereochemistry (Oakes et al., 1999)(Makowski et al., 2013)(Agius et al., 1991) were critical to AYNE's ability to inhibit FAO and induce AML cytotoxicity in vitro and in vivo. The natural products ara- $\mathrm{C}$ and daunorubicin, the backbones of induction therapy, have served as templates for numerous synthetic nucleoside analogues and anthracyclines, respectively, under clinical investigation for AML therapy (Lichtman, 2013). Similarly, the identification of AYNE's critical structural moieties and molecular target presented in this thesis allow for more potent 
VLCAD inhibitors to be developed using AYNE as a structural template. Taken together, chapter 6 demonstrated the structural elements required for AML cytotoxicity by AYNE; future studies modulating FAO activity should utilize synthetic AYNE to eliminate yield variations associated with the extraction of AYNE directly from avocadoes (Salazar-López et al., 2020)(RodríguezLópez et al., 2017).

\subsection{Limitations And Future Directions}

A number of experiments proposed below will further demonstrate the preclinical efficacy of pharmacological VLCAD targeting in AML. The sensitivity of AML cell lines and primary patient-derived samples to FAO inhibition at VLCAD was dependent on both enzyme target expression and the ability to upregulate compensatory metabolism. The AML (Samudio et al., 2010)(Kuntz et al., 2017) and hematopoietic (Filippi \& Ghaffari, 2019)(Ito et al., 2019) compartments are stratified into metabolically heterogeneous subpopulations with varying capacities to proliferate, generate colonies, and engraft. For example, primitive AML cells in the CD34+/CD38- as well as the CD34+/CD38-/CD90+/CD45RA- compartments demonstrated selfrenewal, engraftment capacity, and the ability to reconstitute the AML population in murine bone marrow. Occurring at a rate of one to 250,000 blood cells, these primitive cells are quiescent (Bonnet \& Dick, 1997)(Hope et al., 2004) and less metabolically flexible (Lagadinou et al., 2013).

VLCAD activity and protein levels of bulk primary samples can be quantified prior to metabolomics analysis using the protocols outlined in chapter 5. Cell samples could also be isolated into subpopulations with high and low VLCAD activity and expression to further demonstrate that AYNE targets VLCAD in AML. Due to the metabolic heterogeneity in both AML and hematopoiesis, future studies should conduct stable isotope tracing experiments, established 
in chapter 5, on additional primary AML and normal MNC samples with varying levels of VLCAD as well as the quiescent and non-quiescent subpopulations.

Currently, the primary cause of death in AML patients is relapse caused by chemo-resistant cells surviving induction chemotherapeutics (Farge et al., 2017)(Stevens et al., 2020). While chapter 4 determined that avo-B synergized with AML induction chemotherapeutics in vitro, additional in vivo experiments are required to demonstrate combination synergy at reducing relapse. Sequential patient derived xenograft studies (Stevens et al., 2020) with cells derived from an AML patient experiencing relapse should be conducted with induction chemotherapeutics in combination with AYNE to assess the long term engraftment capacity of AML cells. Alternatively, resistant AML cells can be generated by mimicking an induction therapy regimen in dose and timing on mice carrying de novo AML patient cells (Boyd et al., 2018). The surviving and de novo AML cells can be compared for alterations in VLCAD expression prior to combination therapy with AYNE. VLCAD upregulation in chemo-resistant relapse initiating cells would further demonstrate the pre-clinical role of VLCAD in leukemia FAO. A decrease in engrafted AML cells after sequential engraftments would suggest that FAO inhibition by AYNE in combination with current induction therapy decreases AML long term engraftment capacity.

Along with in vivo studies, in vitro models more accurately modelling the bone marrow and adipose tissue, both niches of LSCs and HSCs, should be conducted to investigate the role of FAO in environmental interactions. Within these niches, there is extensive and complex communication between normal hematopoietic, AML, and adjacent stromal cells. AML cells release inflammatory cytokines to re-model the surrounding bone marrow and adipose niches to simultaneously support leukemia progression and disrupt normal hematopoietic development (Ye et al., 2016)(Duarte et al., 2018). Adjacent stromal cells can also transfer substrates, such as fat 
(Shafat et al., 2017), and functional mitochondria (Moschoi et al., 2016) into AML cells, increasing leukemia FAO to confer resistance towards induction chemotherapy. Co-culturing AML cell lines and primary samples with human-derived adipocytes or bone marrow stromal cells in vitro will more accurately reflect the physiological conditions found in the adipose and bone marrow niches where relapse initiates (Shafat et al., 2017).

Chapter 5 focussed on the disruption of AML mitochondrial metabolism following VLCAD inhibition, namely the loss of FAO contribution to the TCA cycle and to OXPHOS. Enzymes of the long chain FAO, TCA, and ETC pathways are arranged in close proximity and organized into respiratory super complexes to optimize the flow of metabolites and expedite electron transfer to ETC complexes, fueling OXPHOS (Wang et al., 2010). Previously, disruption of the respiratory super complex hindered ETC complex activity and kill AML cells (Mirali et al., 2020). While disruption of the respiratory super complex may partially contribute to the observed mitochondrial dysfunction described in chapter 5, AYNE-induced FAO inhibition could be attenuated by the supplementation of a medium chain fat, while the activities of ETC CI and CII were unaffected, suggesting that the primary metabolic target of AYNE is long chain FAO and not the respiratory super complex. Future experiments involving VLCAD knockdown should delineate the factors contributing to the metabolic dysfunction as being primarily caused by the loss of long chain FAO and not by potential disruption of the respiratory super complex. Site directed mutagenesis targeting VLCAD's catalytic glutamic acid residue could allow for proper folding of VLCAD into the respiratory super complex while inhibiting FAO; this experiment would determine which metabolic effects are due to the loss of the folded VLCAD in architecture of the respiratory super complex compared to the loss of long chain FAO (Schiff et al., 2013). 


\subsection{Conclusion}

In conclusion, the work completed in this thesis uncovered VLCAD as a novel anti-AML target, expanding upon numerous studies demonstrating that FAO is critical to AML mitochondrial metabolism. Genetic knockdown and pharmacological inhibition of VLCAD by AYNE selectively induced death of AML cells, while sparing normal hematopoietic cells in vitro and in vivo. This thesis demonstrated the pre-clinical efficacy of VLCAD inhibition which exploited the abnormal mitochondrial phenotype in AML, characterized by an overreliance on FAO-driven oxidative metabolism and the lack of capacity to upregulate compensatory metabolism. 


\subsection{Chapter 8 References}

Agius, L., Meredith, E. J., \& Sherratt, H. S. A. (1991). Stereospecificity of the inhibition by etomoxir of fatty acid and cholesterol synthesis in isolated rat hepatocytes. Biochemical Pharmacology, 42(9), 1717-1720.

Ahmed, N., Kermanshahi, B., Ghazani, S. M., Tait, K., Tcheng, M., Roma, A., Callender, S. P., Smith, R. W., Tam, W., Wettig, S. D., Rogers, M. A., Marangoni, A. G., \& Spagnuolo, P. A. (2020). Avocado-derived polyols for use as novel co-surfactants in low energy self-emulsifying microemulsions. Scientific Reports, 10(1), 1-14.

Ahmed, N., Smith, R. W., Henao, J. J. A., Stark, K. D., \& Spagnuolo, P. A. (2018). Analytical method to detect and quantify avocatin B in hass avocado seed and pulp matter. Journal of Natural Products, 81(4), 818-824.

Ahmed, N., Tcheng, M., Roma, A., Buraczynski, M., Jayanth, P., Rea, K., Akhtar, T. A., \& Spagnuolo, P. A. (2019). Avocatin B protects against lipotoxicity and improves insulin sensitivity in diet-induced obesity. Molecular Nutrition and Food Research, 63(24), 1-10.

Akutsu, M., Furukawa, Y., Tsunoda, S., Izumi, T., Ohmine, K., \& Kano, Y. (2002). Scheduledependent synergism and antagonism between methotrexate and cytarabine against human leukemia cell lines in vitro. Leukemia, 16(1), 1808-1817.

Baccelli, I., Gareau, Y., Lehnertz, B., Gingras, S., Spinella, J. F., Corneau, S., Mayotte, N., Girard, S., Frechette, M., Blouin-Chagnon, V., Leveillé, K., Boivin, I., MacRae, T., Krosl, J., Thiollier, C., Lavallée, V. P., Kanshin, E., Bertomeu, T., Coulombe-Huntington, J., ... Sauvageau, G. (2019). Mubritinib Targets the Electron Transport Chain Complex I and Reveals the Landscape of OXPHOS Dependency in Acute Myeloid Leukemia. Cancer Cell, 36(1), 84-99.e8.

Bartlett, K., \& Eaton, S. (2004). Mitochondrial $\beta$-oxidation. European Journal of Biochemistry, 271(1), 462-469.

Bonnet, D., \& Dick, J. E. (1997). Human acute myeloid leukemia is organized as a hierarchy that originates from a primitive hematopoietic cell. Nature Medicine, 3(7), 730-737.

Boyd, A. L., Aslostovar, L., Reid, J., Ye, W., Tanasijevic, B., Porras, D. P., Shapovalova, Z., Almakadi, M., Foley, R., Leber, B., Xenocostas, A., \& Bhatia, M. (2018). Identification of chemotherapy-induced leukemic-regenerating cells reveals a transient vulnerability of human AML recurrence. Cancer Cell, 34(3), 483-498.

Brooke, D. G., Shelley, E. J., Roberts, C. G., Denny, W. A., Sutherland, R. L., \& Butt, A. J. (2011). Synthesis and in vitro evaluation of analogues of avocado-produced toxin $(+)-(\mathrm{R})$-persin in human breast cancer cells. Bioorganic and Medicinal Chemistry, 19(23), 7033-7043.

Certo, M., Moore, V. D. G., Nishino, M., Wei, G., Korsmeyer, S., Armstrong, S. a., \& Letai, A. (2006). Mitochondria primed by death signals determine cellular addiction to antiapoptotic BCL2 family members. Cancer Cell, 9(5), 351-365.

Chao, M. P., Seita, J., \& Weissman, I. L. (2008). Establishment of a normal hematopoietic and leukemia stem cell hierarchy. Cold Spring Harbor Symposia on Quantitative Biology, 73, 439- 
449.

Chou, T. C. (2010). Drug combination studies and their synergy quantification using the ChouTalalay method. Cancer Research, 70(2), 440-446.

Cunha, V. L. S., Liu, X., Lowary, T. L., \& O’Doherty, G. A. (2019). De novo asymmetric synthesis of avocadyne, avocadene, and avocadane stereoisomers. Journal of Organic Chemistry, 84(23), $15718-15725$.

Daver, N., Wei, A. H., Pollyea, D. A., Fathi, A. T., Vyas, P., \& DiNardo, C. D. (2020). New directions for emerging therapies in acute myeloid leukemia: the next chapter. Blood Cancer Journal, 10(107), 1-12.

Duarte, D., Hawkins, E. D., \& Celso, C. Lo. (2018). The interplay of leukemia cells and the bone marrow microenvironment. Blood, 131(14), 1507-1511.

Farge, T., Saland, E., de Toni, F., Aroua, N., Hosseini, M., Perry, R., Bosc, C., Sugita, M., Stuani, L., Fraisse, M., Scotland, S., Larrue, C., Boutzen, H., Feliu, V., Nicolau-Travers, M. L., CassantSourdy, S., Broin, N., David, M., Serhan, N., ... Sarry, J. E. (2017). Chemotherapy-Resistant Human Acute Myeloid Leukemia Cells Are Not Enriched for Leukemic Stem Cells but Require Oxidative Metabolism. Cancer Discovery, 7(7), 716-735.

Filippi, M. D., \& Ghaffari, S. (2019). Mitochondria in the maintenance of hematopoietic stem cells: New perspectives and opportunities. Blood, 133(18), 1943-1952.

Fromenty, B., \& Pessayre, D. (1995). Inhibition of mitochondrial beta-oxidation as a mechanism of hepatotoxicity. Pharmacology and Therapeutics, 67(1), 101-154.

German, N. J., Yoon, H., Yusuf, R. Z., Murphy, J. P., Lydia, W. S., Laurent, G., Haas, W., Satterstrom, F. K., Guarnerio, J., Santos, D., Pandolfi, P. P., Beck, A. H., Gygi, S. P., David, T., Jr, W. G. K., \& Haigis, M. C. (2016). PHD3 Loss in Cancer Enables Metabolic Reliance on Fatty Acid Oxidation via Deactivation of ACC2. Molecular Cell, 63(6), 1006-1020.

Gotoh, N., Moroda, K., Watanabe, H., Yoshinaga, K., Tanaka, M., Mizobe, H., Ichioka, K., Tokairin, S., \& Wada, S. (2008). Metabolism of odd-numbered fatty acids and even-numbered fatty acids in mouse. Journal of Oleo Science, 57(5), 293-299.

Hao, X., Gu, H., Chen, C., Huang, D., Zhao, Y., Xie, L., Zou, Y., Shu, H. S., Zhang, Y., He, X., Lai, X., Zhang, X., Zhou, B. O., Zhang, C. C., Chen, G. Q., Yu, Z., Yang, Y., \& Zheng, J. (2019). Metabolic Imaging Reveals a Unique Preference of Symmetric Cell Division and Homing of Leukemia-Initiating Cells in an Endosteal Niche. Cell Metabolism, 29(4), 950-965.

Havel, R. J. (1987). Lipid transport function of lipoproteins in blood plasma. American Journal of Physiology - Endocrinology and Metabolism, 253(16), E1-E5.

Heiden, M. G. Vander, Cantley, L. C., \& Thompson, C. B. (2009). Understanding the Warburg effect: the metabolic requirements of cell proliferation. Science, 324(5930), 1029-1033.

Holubarsch, C. J. F., Rohrbach, M., Karrasch, M., Boehm, E., Polonski, L., Ponikowski, P., \& Rhein, S. (2007). A double-blind randomized multicentre clinical trial to evaluate the efficacy and safety of two doses of etomoxir in comparison with placebo in patients with moderate congestive heart failure: the ERGO (etomoxir for the recovery of glucose oxidation)study. Clinical Science, 


\section{3(4), 205-212.}

Holyoake, T. L., \& Alcorn, M. J. (1994). CD34+ positive haemopoietic cells: biology and clinical applications. Blood Reviews, 8(1), 113-124.

Hope, K. J., Jin, L., \& Dick, J. E. (2004). Acute myeloid leukemia originates from a hierarchy of leukemic stem cell classes that differ in self-renewal capacity. Nature Immunology, 5(7), 738-743.

Horton, S. J., \& Huntly, B. J. P. (2012). Recent advances in acute myeloid leukemia stem cell biology. Haematologica, 97(7), 966-974.

Houten, S. M., \& Wanders, R. J. A. (2010). A general introduction to the biochemistry of mitochondrial fatty acid $\beta$-oxidation. Journal of Inherited Metabolic Disease, 33(5), 469-477.

Huntly, B. J. P., \& Gilliland, D. G. (2005). Leukaemia stem cells and the evolution of cancer-stemcell research. Nature Reviews Cancer, 5(April), 311-322.

Hutter, E., Unterluggauer, H., Garedew, A., Jansendurr, P., \& Gnaiger, E. (2006). High-resolution respirometry-a modern tool in aging research. Experimental Gerontology, 41(1), 103-109.

Huynh, F. K., Green, M. F., Koves, T. R., \& Hirschey, M. D. (2014). Measurement of fatty acid oxidation rates in animal tissues and cell lines. Methods in Enzymology, 542(1), 391-405.

Ishii, T., Okai, T., Iwatani-Yoshihara, M., Mochizuki, M., Unno, S., Kuno, M., Yoshikawa, M., Shibata, S., Nakakariya, M., Yogo, T., \& Kawamoto, T. (2017). CETSA quantitatively verifies in vivo target engagement of novel RIPK1 inhibitors in various biospecimens. Scientific Reports, $7(1), 1-14$.

Ito, Keisuke, Bernardi, R., Morotti, A., Matsuoka, S., Saglio, G., Ikeda, Y., Rosenblatt, J., Avigan, D. E., Teruya-Feldstein, J., \& Pandolfi, P. P. (2008). PML targeting eradicates quiescent leukaemia-initiating cells. Bone, 23(1), 1-7.

Ito, Kyoko, Bonora, M., \& Ito, K. (2019). Metabolism as master of hematopoietic stem cell fate. International Journal of Hematology, 109(1), 18-27.

Jacque, N., Ronchetti, A. M., Larrue, C., Meunier, G., Birsen, R., Willems, L., Saland, E., Decroocq, J., Maciel, T. T., Lambert, M., Poulain, L., Hospital, M. A., Sujobert, P., Joseph, L., Chapuis, N., Lacombe, C., Moura, I. C., Demo, S., Sarry, J. E., ... Bouscary, D. (2015). Targeting glutaminolysis has antileukemic activity in acute myeloid leukemia and synergizes with BCL-2 inhibition. Blood, 126(11), 1346-1356.

Jonas, B. A., \& Pollyea, D. A. (2019). How we use venetoclax with hypomethylating agents for the treatment of newly diagnosed patients with acute myeloid leukemia. Leukemia, 33(12), 27952804.

Jones, C. L., Stevens, B. M., D’Alessandro, A., Reisz, J. A., Culp-Hill, R., Nemkov, T., Pei, S., Khan, N., Adane, B., Ye, H., Krug, A., Reinhold, D., Smith, C., DeGregori, J., Pollyea, D. A., \& Jordan, C. T. (2018). Inhibition of amino acid metabolism selectively targets human leukemia stem cells. Cancer Cell, 34(5), 724-740.

Jose, C., Bellance, N., \& Rossignol, R. (2011). Choosing between glycolysis and oxidative phosphorylation: A tumor's dilemma? Biochimica et Biophysica Acta (BBA) - Bioenergetics, 
1807(6), 552-561.

Jun, S. A., Sepiashvili, L., Kislinger, T., \& Minden, M. D. (2011). Investigating the potential use of L-asparaginase in myeloid leukemia. Blood, 118(21), 3641.

Kantor, P. F., Lucien, a, Kozak, R., \& Lopaschuk, G. D. (2000). The antianginal drug trimetazidine shifts cardiac energy metabolism from fatty acid oxidation to glucose oxidation by inhibiting mitochondrial long-chain 3-ketoacyl coenzyme A thiolase. Circulation Research, 86, $580-588$.

Karabatsiakis, A., Böck, C., Salinas-Manrique, J., Kolassa, S., Calzia, E., Dietrich, D. E., \& Kolassa, I. T. (2014). Mitochondrial respiration in peripheral blood mononuclear cells correlates with depressive subsymptoms and severity of major depression. Translational Psychiatry, 4(January), e397-e404.

Kashman, Y., Neeman, I., \& Lifshitz, A. (1969). New compounds from avocado pear. Tetrahedron, 25(1), 4617-4631.

Kaur, G., \& Dufour, J. M. (2012). Cell lines: valuable tools or useless artifacts. Spermatogenesis, $2(1), 1-5$.

Khwaja, A., Bjorkholm, M., Gale, R. E., Levine, R. L., Jordan, C. T., Ehninger, G., Bloomfield, C. D., Estey, E., Burnett, A., Cornelissen, J. J., Scheinberg, D. A., Bouscary, D., \& Linch, D. C. (2016). Acute myeloid leukemia. Nature Reviews Disease Primers, 2(1), 1-22.

Kuntz, E. M., Baquero, P., Michie, A. M., Dunn, K., Tardito, S., Holyoake, T. L., Helgason, G. V., \& Gottlieb, E. (2017). Targeting mitochondrial oxidative phosphorylation eradicates therapyresistant chronic myeloid leukemic stem cells. Nature Medicine, 23(10), 1234-1240.

Lagadinou, E. D., Sach, A., Callahan, K., Rossi, R. M., Neering, S. J., Minhajuddin, M., Ashton, J. M., Pei, S., Grose, V., O’Dwyer, K. M., Liesveld, J. L., Brookes, P. S., Becker, M. W., \& Jordan, C. T. (2013). BCL-2 inhibition targets oxidative phosphorylation and selectively eradicates quiescent human leukemia stem cells. Cell Stem Cell, 12(3), 329-341.

Lamba, J. K. (2009). Genetic factors influencing cytarabine therapy. Pharmacogenomics, 10(10), $1657-1674$.

Lee, E. A., Angka, L., Rota, S. G., Hanlon, T., Mitchell, A., Hurren, R., Wang, X. M., Gronda, M., Boyaci, E., Bojko, B., Minden, M., Sriskanthadevan, S., Datti, A., Wrana, J. L., Edginton, A., Pawliszyn, J., Joseph, J. W., Quadrilatero, J., Schimmer, A. D., \& Spagnuolo, P. a. (2015). Targeting mitochondria with avocatin B induces selective leukemia cell death. Cancer Research, 75(12), 2478-2488.

Lichtman, M. A. (2013). A historical perspective on the development of the cytarabine (7days) and daunorubicin (3days) treatment regimen for acute myelogenous leukemia: 2013 the 40th anniversary of 7+3. Blood Cells, Molecules, and Diseases, 50(2), 119-130.

Liu, F. T., Agrawal, S. G., Movasaghi, Z., Wyatt, P. B., Rehman, I. U., Gribben, J. G., Newland, A. C., \& Jia, L. (2008). Dietary flavonoids inhibit the anticancer effects of the proteasome inhibitor bortezomib. Blood, 112(9), 3835-3846.

Löwenberg, B., Downing, J. R., \& Burnett, A. (2011). Acute Myeloid Leukemia. New England 
Journal of Medicine, 341(14), 1051-1062.

Luengo, A., Li, Z., Y.Gui, D., Sullivan, L. B., Zagorulya, M., Do, B. T., Ferreira, R., Naamati, A., Ali, A., Lewis, C. A., Thomas, C. J., Spranger, S., Matheson, N. J., \& Heiden, M. G. Vander. (2021). Increased demand for NAD+ relative to ATP drives aerobic glycolysis. Molecular Cell, 81(1), 691-707.

Lunt, S. Y., \& Vander Heiden, M. G. (2011). Aerobic glycolysis: meeting the metabolic requirements of cell proliferation. Annu. Rev. Cell Dev. Biol., 27(1), 441-464.

MacInnes, A., Fairman, D. a, Binding, P., Rhodes, J. A., Wyatt, M. J., Phelan, A., Haddock, P. S., \& Karran, E. H. (2003). The antianginal agent trimetazidine does not exert its functional benefit via inhibition of mitochondrial long-chain 3-ketoacyl coenzyme A thiolase. Circulation Research, 93(3), e26-e32.

Makowski, K., Mera, P., Paredes, D., Herrero, L., Ariza, X., Asins, G., Hegardt, F. G., Garcia, J., \& Serra, D. (2013). Differential pharmacologic properties of the two C75 enantionmers (+)-C75 is a strong anorectic drug; (-)-C75 has antitumor activity. Chirality, 24(1), 281-287.

Makrecka-Kuka, M., Krumschnabel, G., \& Gnaiger, E. (2015). High-Resolution Respirometry for Simultaneous Measurement of Oxygen and Hydrogen Peroxide Fluxes in Permeabilized Cells, Tissue Homogenate and Isolated Mitochondria. Biomolecules, 5(3), 1319-1338.

Martínez-Reyes, I., \& Chandel, N. S. (2020). Mitochondrial TCA cycle metabolites control physiology and disease. Nature Communications, 11(102), 1-11.

McAndrew, R. P., Wang, Y., Mohsen, A. W., He, M., Vockley, J., \& Kim, J. J. P. (2008). Structural basis for substrate fatty acyl chain specificity: Crystal structure of human very-long-chain acylCoA dehydrogenase. Journal of Biological Chemistry, 283(14), 9435-9443.

Mirali, S., Aaron, B., Voisin, V., Xu, C., St-Germain, J., Sharon, D., Hoff, F. W., Qiu, Y., Hurren, R., Gronda, M., Jitkova, Y., Xu, G. W., Nachmias, B., MacLean, N., Wang, X., Arruda, A., Minden, M. D., Horton, T. M., Kornblau, S. M., ... Schimmer, A. D. (2020). The Mitochondrial Protease, Neurolysin (NLN), Regulates Respiratory Chain Complex and Supercomplex Formation and Is Necessary for AML Viability. Science Translational Medicine, 12(8264), 1-16.

Modernelli, A., Naponelli, V., Giovanna Troglio, M., Bonacini, M., Ramazzina, I., Bettuzzi, S., \& Rizzi, F. (2015). EGCG antagonizes bortezomib cytotoxicity in prostate cancer cells by an autophagic mechanism. Scientific Reports, 5(15270), 1-11.

Moffat, J., Grueneberg, D. A., Yang, X., Kim, S. Y., Kloepfer, A. M., Hinkle, G., Piqani, B., Eisenhaure, T. M., Luo, B., Grenier, J. K., Carpenter, A. E., Foo, S. Y., Stewart, S. A., Stockwell, B. R., Hacohen, N., Hahn, W. C., Lander, E. S., Sabatini, D. M., \& Root, D. E. (2006). A Lentiviral RNAi Library for Human and Mouse Genes Applied to an Arrayed Viral High-Content Screen. Cell, 124(6), 1283-1298.

Molina, J. R., Sun, Y., Protopopova, M., Gera, S., Bandi, M., Bristow, C., McAfoos, T., Morlacchi, P., Ackroyd, J., Agip, A. N. A., Al-Atrash, G., Asara, J., Bardenhagen, J., Carrillo, C. C., Carroll, C., Chang, E., Ciurea, S., Cross, J. B., Czako, B., ... Marszalek, J. R. (2018). An inhibitor of oxidative phosphorylation exploits cancer vulnerability. Nature Medicine, 24(7), 1036-1046.

Moschoi, R., Imbert, V., Nebout, M., Chiche, J., Mary, D., Prebet, T., Saland, E., Castellano, R., 
Pouyet, L., Collette, Y., Vey, N., Chabannon, C., Recher, C., Sarry, J.-E., Alcor, D., Peyron, J.-F., \& Greissinger, E. (2016). Protective mitochondrial transfer from bone marrow stromal cells to acute myeloid leukemic cells during chemotherapy. Blood, 128(2), 253-264.

Muerhoffs, A. S., Williams, D. E., Reichll, N., Cajacob, C. A., \& De, P. R. O. (1989). Prostaglandin and fatty acid omega- and (omega-1)-oxidation in rabbit lung acetylenic fatty acid mechanism-based inactivators as specific inhibitors. Journal of Biological Chemistry, 264(2), 749-756.

Muus, P., Van den Bogert, C., De Vries, H., Pennings, a, Holtrop, M., \& Haanen, C. (1991). 1beta-D-arabinofuranosylcytosine (Ara-C) enhances mitochondrial activities in human leukaemic cells. Br J Cancer, 64(1), 29-34.

Nakahara, F., Weiss, C. N., \& Ito, K. (2014). The role of PML in hematopoietic and leukemic stem cell maintenance. International Journal of Hematology, 100(1), 18-26.

Nash, D. T., \& Nash, S. D. (2008). Ranolazine for chronic stable angina. The Lancet, 372(9646), $1335-1341$.

Néeman, I., Lifshitz, A., \& Kashman, Y. (1970). New antibacterial agent isolated from the avocado pear. Applied Microbiology, 19(3), 470-473.

Oakes, N. D., Kjellstedt, A., Forsberg, G. B., Clementz, T., Camejo, G., Furler, S. M., Kraegen, E. W., Ölwegård-Halvarsson, M., Jenkins, A. B., \& Ljung, B. (1999). Development and initial evaluation of a novel method for assessing tissue-specific plasma free fatty acid utilization in vivo using (R)-2- bromopalmitate tracer. Journal of Lipid Research, 40(6), 1155-1169.

Oberlies, N. H., Rogers, L. L., Martin, J. M., \& McLaughlin, J. L. (1998). Cytotoxic and insecticidal constituents of the unripe fruit of Persea americana. Journal of Natural Products, 61(6), 781-785.

Ojuka, E., Andrew, B., Bezuidenhout, N., George, S., Maarman, G., Madlala, H. P., Mendham, A., \& Osiki, P. O. (2016). Measurement of beta-oxidation capacity of biological samples by respirometry: A review of principles and substrates. American Journal of Physiology Endocrinology And Metabolism, 310(9), 715-723.

Okun, J., Kölker, S., Schulze, A., Kohlmuller, D., Olgemoller, K., Linder, M., Hoffmann, G. F., Wanders, R. J., \& Mayatepek, E. (2002). A method for quantitative acylcarnitine profiling in human skin fibroblasts using unlabelled palmitic acid: diagnosis of fatty acid oxidation disorders and differentiation. Biochimica et Biophysica Acta, 1584(1), 91-98.

Olson, K. A., Schell, J. C., \& Rutter, J. (2016). Pyruvate and Metabolic Flexibility: Illuminating a Path Toward Selective Cancer Therapies. Trends in Biochemical Sciences, 41(3), 219-230.

Pardee, T. S., Anderson, R. G., Pladna, K. M., Isom, S., Ghiraldeli, L. P., Miller, L. D., Chou, J. W., Jin, G., Zhang, W., Ellis, L. R., Berenzon, D., Howard, D. S., Hurd, D. D., Manuel, M., Dralle, S., Lyerly, S., \& Powell, B. L. (2018). A phase I study of CPI-613 in combination with high-dose cytarabine and mitoxantrone for relapsed or refractory acute myeloid leukemia. Clinical Cancer Research, 24(9), 2060-2073.

Pereira, D. S., Dorrell, C., Ito, C. Y., Gan, O. I., Murdoch, B., Rao, V. N., Zou, J.-P., Reddy, E. S. P., \& Dick, J. E. (1998). Retroviral transduction of TLS-ERG initiates a leukemogenic program in 
normal human hematopoietic cells. Proceedings of the National Academy of Sciences, 95(July), 8239-8244.

Pollyea, D. A., Pratz, K., Letai, A., Jonas, B. A., Wei, A. H., Pullarkat, V., Konopleva, M., Thirman, M. J., Arellano, M., Becker, P. S., Chyla, B., Hong, W. J., Jiang, Q., Potluri, J., \& DiNardo, C. D. (2020). Venetoclax with azacitidine or decitabine in patients with newly diagnosed acute myeloid leukemia: long term follow-up from a phase $1 \mathrm{~b}$ study. American Journal of Hematology, 96(2), 208-217.

Pollyea, D. A., Stevens, B. M., Jones, C. L., Winters, A., Pei, S., Minhajuddin, M., D’Alessandro, A., Culp-Hill, R., Riemondy, K. A., Gillen, A. E., Hesselberth, J. R., Abbott, D., Schatz, D., Gutman, J. A., Purev, E., Smith, C., \& Jordan, C. T. (2018). Venetoclax with azacitidine disrupts energy metabolism and targets leukemia stem cells in patients with acute myeloid leukemia. Nature Medicine, 24(12), 1859-1866.

Ramaswamy, S. (2007). Rational design of cancer-drug combinations. NEngl J Med, 357(3), 299300 .

Reinisch, A., Chan, S. M., Thomas, D., \& Majeti, R. (2015). Biology and Clinical Relevance of Acute Myeloid Leukemic Stem Cells. Seminal Hematology, 52(3), 150-164.

Reitman, Z. J., \& Yan, H. (2010). Isocitrate dehydrogenase 1 and 2 mutations in cancer: Alterations at a crossroads of cellular metabolism. Journal of the National Cancer Institute, 102(13), 932941.

Rodríguez-López, C. E., Hernández-Brenes, C., Treviño, V., \& Díaz de la Garza, R. I. (2017). Avocado fruit maturation and ripening: dynamics of aliphatic acetogenins and lipidomic profiles from mesocarp, idioblasts and seed. BMC Plant Biology, 17(159), 1-23.

Saito, Y., Chapple, R. H., Lin, A., Kitano, A., \& Nakada, D. (2015). AMPK Protects LeukemiaInitiating Cells in Myeloid Leukemias from Metabolic Stress in the Bone Marrow. Cell Stem Cell, 17(5), 585-596.

Salazar-López, N. J., Domínguez-Avila, J. A., Yahia, E. M., Belmonte-Herrera, B. H., WallMedrano, A., Montalvo-González, E., \& González-Aguilar, G. A. (2020). Avocado fruit and byproducts as potential sources of bioactive compounds. Food Research International, 138(12), 109774.

Samudio, I., Harmancey, R., Fiegl, M., Kantarjian, H., Konopleva, M., Korchin, B., Kaluarachchi, K., Bornmann, W., Duvvuri, S., Taegtmeyer, H., \& Andreeff, M. (2010). Pharmacologic inhibition of fatty acid oxidation sensitizes human leukemia cells to apoptosis induction. Journal of Clinical Investigation, 120(1), 142-156.

Sarma, N. J., Takeda, A., \& Yaseen, N. R. (2010). Colony forming cell (CFC) assay for human hematopoietic cells. Journal of Visualized Experiments, 46(2195), 1-5.

Schiff, M., Mohsen, A.-W., Karunanidhi, A., McCracken, E., Yeasted, R., \& Vockley, J. (2013). Molecular and Cellular Pathology of Very-Long-Chain Acyl-CoA Dehydrogenase Deficiency. Molecular Genetics and Metabolism, 109(1), 21-27.

Seneviratne, A. K., Xu, M., Henao, J. J. A., Fajardo, V. A., Hao, Z., Voisin, V., Xu, G. W., Hurren, R., Kim, S., Maclean, N., Wang, X., Gronda, M., Jeyaraju, D., Jitkova, Y., Ketela, T., Sharon, D., 
Thomas, G., Chouinard-watkins, R., \& James, R. (2020). Stemness by Modulating Intracellular Levels of Phospholipids. 24(4), 621-636.

Shafat, M. S., Oellerich, T., Mohr, S., Robinson, S. D., Edwards, D. R., Marlein, C. R., Piddock, R. E., Fenech, M., Zaitseva, L., Abdul-Aziz, A., Turner, J., Watkins, J. A., Lawes, M., Bowles, K. M., \& Rushworth, S. A. (2017). Leukemic blasts program bone marrow adipocytes to generate a protumoral microenvironment. Blood, 129(10), 1320-1332.

Sharabi, A., \& Tsokos, G. C. (2020). T cell metabolism: new insights in systemic lupus erythematosus pathogenesis and therapy. Nature Reviews Rheumatology, 16(2), 100-112.

Sharon, D., Cathelin, S., Mirali, S., Di Trani, J. M., Yanofsky, D. J., Keon, K. A., Rubinstein, J. L., Schimmer, A. D., Ketela, T., \& Chan, S. M. (2019). Inhibition of mitochondrial translation overcomes venetoclax resistance in AML through activation of the integrated stress response. Science Translational Medicine, 11(2863), 1-15.

Shi, J., Fu, H., Jia, Z., He, K., Fu, L., \& Wang, W. (2016). High Expression of CPT1A Predicts Adverse Outcomes: A Potential Therapeutic Target for Acute Myeloid Leukemia. EBioMedicine, 14(1), 55-64.

Shibata, R., Gotoh, N., Kubo, A., Kanda, J., Nagai, T., Mizobe, H., Yoshinaga, K., Kojima, K., Watanabe, H., \& Wada, S. (2012). Comparison of catabolism rate of fatty acids to carbon dioxide in mice. European Journal of Lipid Science and Technology, 114(12), 1340-1344.

Shipley, J. L., \& Butera, J. N. (2009). Acute myelogenous leukemia. Experimental Hematology, 37(6), 649-658.

Škrtić, M., Sriskanthadevan, S., \& Jhas, B. (2011). Inhibition of mitochondrial translation as a therapeutic strategy for human acute myeloid leukemia. Cancer Cell, 20(5), 674-688.

Spagnuolo, P. A., Hurren, R., Gronda, M., Maclean, N., Datti, A., Basheer, A., Lin, F. H., Wang, X., Wrana, J., \& Schimmer, A. D. (2013). Inhibition of intracellular dipeptidyl peptidases 8 and 9 enhances parthenolide's anti-leukemic activity. Leukemia, 27(6), 1236-1244.

Sriskanthadevan, S., Jeyaraju, D. V., Chung, T. E., Prabha, S., Xu, W., Skrtic, M., Jhas, B., Hurren, R., Gronda, M., Wang, X., Jitkova, J., Sukhai, M. A., Lin, F.-H., Maclean, N., Laister, R., Board, C. A., Mullen, P. J., Xie, S., Penn, L. Z., ... Schimmer, A. D. (2015). AML cells have low spare reserve capacity in their respiratory chain that renders them susceptible to oxidative metabolic stress. Blood, 125(13), 2120-2130.

Stevens, B. M., Jones, C. L., Pollyea, D. A., Culp-Hill, R., D.Alessandro, A., Winters, A., Krug, A., Abbott, D., Goosman, M., Pei, S., Ye, H., Gillen, A. E., Becker, M. W., Savona, M. R., Smith, C., \& Jordan, C. T. (2020). Fatty acid metabolism underlies venetoclax resistance in acute myeloid leukemia stem cells. Nature Cancer, 1(1), 1176-1187.

Sugiyama, T., Sato, A., \& Yamashita, K. (1982). Synthesis of all four stereoisomers of antibacterial component of avocado. Agricultural and Biological Chemistry, 46(2), 481-485.

Tabe, Y., Saitoh, K., Yang, H., Sekihara, K., Yamatani, K., Ruvolo, V., Taka, H., Kaga, N., Kikkawa, M., Arai, H., Miida, T., Andreeff, M., Spagnuolo, P. A., \& Konopleva, M. (2018). Inhibition of FAO in AML co-cultured with BM adipocytes: mechanisms of survival and chemosensitization to cytarabine. Scientific Reports, 8(1), 16837. 
Thorpe, C., \& Kim, J.-J. P. (1995). Structure and mechanism of action of the acyl-CoA dehydrogenases. FASEB, 9(1), 718-725.

Tucci, S., Herebian, D., Sturm, M., Seibt, A., \& Spiekerkoetter, U. (2012). Tissue-Specific Strategies of the Very-Long Chain Acyl-CoA Dehydrogenase-Deficient (VLCAD-/-) Mouse to Compensate a Defective Fatty Acid $\beta$-Oxidation. PLoS ONE, 7(9), 45429.

van der Windt, G. J. W., Everts, B., Chang, C. H., Curtis, J. D., Freitas, T. C., Amiel, E., Pearce, E. J., \& Pearce, E. L. (2012). Mitochondrial Respiratory Capacity Is a Critical Regulator of CD8 + T Cell Memory Development. Immunity, 36(1), 68-78.

Vockley, J., Burton, B., Berry, G., Longo, N., Philips, J., Sanchez-Valle, A., Chapman, K., Tanpaiboon, P., Grunewald, S., Murphy, E., Lu, X., \& C, J. (2016). Triheptanoin treatment in patients with pediatric cardiomyopathy associated with long chain-fatty acid oxidation disorders. Molecular Genetics and Metabolism, 119(3), 223-231.

Vockley, J., Burton, B., Berry, G., Longo, N., Phlips, J., Sanchez-Valle, A., Chapman, K., Tanpaiboon, P., Grunewald, S., Murphy, E., Lu, X., \& Cataldo, J. (2021). Effects of triheptanoin (UX007) in patients with long-chain fatty acid oxidation disorders: Results from an open-label, long-term extension study. Journal of Molecular Inherited Disease, 44(1), 253-263.

Vockley, J., Mohsen, A.-W. A., Binzak, B., Willard, J., \& Fauq, A. (2004). Mammalian BranchedChain Acyl-CoA Dehydrogenases: Molecular Cloning and Characterization of Recombinant Enzymes. Methods in Enzymology, 324(1989), 241-258.

Wanders, R. J. A., Ruiter, J. P. N., Ijlst, L., Waterham, H. R., \& Houten, S. M. (2010). The enzymology of mitochondrial fatty acid beta-oxidation and its application to follow-up analysis of positive neonatal screening results. Journal of Inherited Metabolic Disease, 33(5), 479-494.

Wang, Y. H., Israelsen, W. J., Lee, D., Yu, V. W. C., Jeanson, N. T., Clish, C. B., Cantley, L. C., Heiden, M. G. Vander, \& Scadden, D. T. (2014). Cell state-specific metabolic dependency in hematopoiesis and leukemogenesis. Cell, 158(6), 1309-1323.

Wang, Y., Mohsen, A. W., Mihalik, S. J., Goetzman, E. S., \& Vockley, J. (2010). Evidence for physical association of mitochondrial fatty acid oxidation and oxidative phosphorylation complexes. Journal of Biological Chemistry, 285(39), 29834-29841.

Wang, Y., Palmfeldt, J., Gregersen, N., Makhov, A. M., Conway, J. F., Wang, M., McCalley, S. P., Basu, S., Alharbi, H., St. Croix, C., Calderon, M. J., Watkins, S., \& Vockley, J. (2019). Mitochondrial fatty acid oxidation and the electron transport chain comprise a multifunctional mitochondrial protein complex. Journal of Biological Chemistry, 294(33), 12380-12391.

Warner, J. K., Wang, J. C. Y., Takenaka, K., Doulatov, S., McKenzie, J. L., Harrington, L., \& Dick, J. E. (2005). Direct evidence for cooperating genetic events in the leukemic transformation of normal human hematopoietic cells. Leukemia, 19(10), 1794-1805.

Wu, Y., Hurren, R., MacLean, N., Gronda, M., Jitkova, Y., Sukhai, M. a., Minden, M. D., \& Schimmer, A. D. (2015). Carnitine transporter CT2 (SLC22A16) is over-expressed in acute myeloid leukemia (AML) and target knockdown reduces growth and viability of AML cells. Apoptosis, 20(8), 1099-1108.

Xiong, D., He, H., James, J., Tokunaga, C., Powers, C., Huang, Y., Osinska, H., Towbin, J. A., 
Purevjav, E., Balschi, J. A., Javadov, S., Jr, F. X. M., Strauss, A. W., \& Khuchua, Z. (2013). Cardiac-specific VLCAD deficiency induces dilated cardiomyopathy and cold intolerance. American Journal of Physiology Heart and Circulation Physiology, 306(3), 2021.

Yang, L., Venneti, S., \& Nagrath, D. (2017). Glutaminolysis: a hallmark of cancer metabolism. Annu. Rev. Biomed. Eng., 19(1), 163-194.

Yap, M. C., Kostiuk, M. A., Martin, D. D. O., Perinpanayagam, M. A., Hak, P. G., Siddam, A., Majjigapu, J. R., Rajaiah, G., Keller, B. O., Prescher, J. A., Wu, P., Bertozzi, C. R., Falck, J. R., $\&$ Berthiaume, L. G. (2010). Rapid and selective detection of fatty acylated proteins using $\omega$ alkynyl-fatty acids and click chemistry. Journal of Lipid Research, 51(6), 1566-1580.

Ye, H., Adane, B., Khan, N., Sullivan, T., Minhajuddin, M., Gasparetto, M., Stevens, B., Pei, S., Balys, M., Ashton, J. M., Klemm, D. J., Woolthuis, C. M., Stranahan, A. W., Park, C. Y., \& Jordan, C. T. (2016). Leukemic stem cells evade chemotherapy by metabolic adaptation to an adipose tissue niche. Cell Stem Cell, 19(1), 23-37.

Zhang, T., Yang, J., Vaikari, V. P., Beckford, J. S., Wu, S., Akhtari, M., \& Alachkar, H. (2020). Apolipoprotein C2 - CD36 Promotes Leukemia Growth and Presents a Targetable Axis in Acute Myeloid Leukemia. Blood Cancer Discovery, 1(1), 198-213.

Zhao, R. Z., Jiang, S., Zhang, L., \& Yu, Z. Bin. (2019). Mitochondrial electron transport chain, ROS generation and uncoupling. International Journal of Molecular Medicine, 44(1), 3-15. 


\subsection{Chapter 9 Permissions}

\subsection{Figure Permissions}

\begin{tabular}{|c|c|}
\hline \multicolumn{2}{|c|}{ Copyright permission for figure 1.2.1 } \\
\hline License Number & 5057200919230 \\
\hline License date & Apr 27, 2021 \\
\hline Licensed Content Publisher & Springer Nature \\
\hline Licensed Content Publication & Nature Reviews Disease Primers \\
\hline Licensed Content Title & Acute myeloid leukaemia \\
\hline Licensed Content Author & Asim Khwaja et al \\
\hline Figures used & Figure 5 \\
\hline Title & $\begin{array}{c}\text { Very long chain fatty acid metabolism is } \\
\text { required in acute } \\
\text { myeloid leukemia }\end{array}$ \\
\hline Institution & University of Guelph \\
\hline Expected Presentation Date & August 2021 \\
\hline
\end{tabular}

\begin{tabular}{|c|c|}
\hline \multicolumn{2}{|c|}{ Copyright permission for figure 1.2.2 } \\
\hline License Number & 5057221416924 \\
\hline License date & Apr 27, 2021 \\
\hline Licensed Content Publisher & Nature Reviews Cancer \\
\hline Licensed Content Publication & Brian J. P. Huntly et al \\
\hline Licensed Content Title & $\begin{array}{l}\text { Leukaemia stem cells and the evolution of } \\
\text { cancer-stem cell research }\end{array}$ \\
\hline Licensed Content Author & Very long chain fatty acid metabolism is \\
Figures used & myeloid leukemia \\
\hline Title & University of Guelph \\
\hline Institution & August 2021 \\
\hline
\end{tabular}




\begin{tabular}{|c|c|}
\hline \multicolumn{2}{|c|}{ Copyright permission for figure 1.4.1 } \\
\hline License Number & 5058790528161 \\
\hline License date & Apr 30, 2021 \\
\hline Licensed Content Publisher & Nature Reviews Rheumatology \\
\hline Licensed Content Publication & $\begin{array}{c}\text { lupus } \\
\text { Licensed Content Title }\end{array}$ \\
& $\begin{array}{c}\text { T cell metabolism: new insights in systemic } \\
\text { erythematosus pathogenesis and therapy }\end{array}$ \\
\hline Licensed Content Author & Amir Sharabi et al \\
\hline Figures used & Figure 1 \\
\hline Title & $\begin{array}{c}\text { Very long chain fatty acid metabolism is } \\
\text { required in acute } \\
\text { myeloid leukemia }\end{array}$ \\
\hline Institution & University of Guelph \\
\hline Expected Presentation Date & August 2021 \\
\hline
\end{tabular}

\begin{tabular}{|c|c|}
\hline \multicolumn{2}{|c|}{ Copyright permission for figure 1.8.1 } \\
\hline License Number & 5060890994947 \\
\hline License date & May 02, 2021 \\
\hline Licensed Content Publisher & John Wiley and Sons \\
\hline Licensed Content Publication & THE FASEB JOURNAL \\
\hline Licensed Content Title & $\begin{array}{l}\text { Structure and mechanism of action of the } \\
\text { Acyl-CoA dehydrogenases }\end{array}$ \\
\hline Licensed Content Author & Colin Thorpe, Jung-Ja P. Kim \\
\hline Figures used & Scheme 3 \\
\hline Title & Very long chain fatty acid metabolism is \\
& required in acute \\
& myeloid leukemia \\
\hline Institution & University of Guelph \\
\hline Expected Presentation Date & August 2021 \\
\hline
\end{tabular}




\subsection{Publication Permissions}

\subsubsection{Chapter 4}

The mitochondria target drug avocatin B synergizes with induction chemotherapeutics to induce leukemia cell death

Author: Matthew Tcheng, , Ismael Samudio, et al

Publication: LEUKEMIA \& LYMPHOMA

Publisher: Taylor \& Francis

Date: Apr 3, 2017

Rights managed by Taylor \& Francis

Thesis/Dissertation Reuse Request

Taylor \& Francis is pleased to offer reuses of its content for a thesis or dissertation free of charge contingent on resubmission of permission request if work is published.

BACK

CLOSE 


\subsubsection{Chapter 5}

Firefox

https://marketplace.copyright.com/rs-ui-web/mp/license/03d14b8f-4db4-...

\begin{tabular}{|c|c|c|c|}
\hline \multicolumn{4}{|c|}{ Elsevier Science \& Technology Journals - License Terms and Conditions } \\
\hline \multicolumn{4}{|c|}{$\begin{array}{l}\text { This is a License Agreement between Matthew Tcheng ("You") and Elsevier Science \& Technology Journals } \\
\text { ("Publisher") provided by Copyright Clearance Center ("CCC"). The license consists of your order details, the terms } \\
\text { and conditions provided by Elsevier Science \& Technology Journals, and the CCC terms and conditions. }\end{array}$} \\
\hline $\begin{array}{l}\text { Order Date } \\
\text { Order license ID } \\
\text { ISSN }\end{array}$ & $\begin{array}{l}\text { 19-May-2021 } \\
1120320-1 \\
1528-0020\end{array}$ & $\begin{array}{l}\text { Type of Use } \\
\text { Publisher } \\
\text { Portion }\end{array}$ & $\begin{array}{l}\text { Republish in a } \\
\text { thesis/dissertation } \\
\text { AMERICAN SOCIETY OF } \\
\text { HEMATOLOGY } \\
\text { Chart/graph/table/figure }\end{array}$ \\
\hline \multicolumn{4}{|c|}{ LICENSED CONTENT } \\
\hline Publication Title & Blood: journal of the & Country & United States of America \\
\hline & $\begin{array}{l}\text { American Society of } \\
\text { Hematology }\end{array}$ & Rightsholder & $\begin{array}{l}\text { Elsevier Science \& } \\
\text { Technology Journals }\end{array}$ \\
\hline Author/Editor & $\begin{array}{l}\text { American Society of } \\
\text { Hematology., HighWire } \\
\text { Press. }\end{array}$ & $\begin{array}{l}\text { Publication Type } \\
\text { URL }\end{array}$ & $\begin{array}{l}\text { e-Journal } \\
\text { http://www.bloodjournal.o }\end{array}$ \\
\hline Date & $01 / 01 / 1946$ & & 'g/ \\
\hline Language & English & & \\
\hline
\end{tabular}

\section{REQUEST DETAILS}

Portion Type
Number of charts /
graphs / tables / figures
requested
Format (select all that
apply)
Who will republish the
content?
Duration of Use
Lifetime Unit Quantity
Rights Requested

Chart/graph/table/figure

18

Print, Electronic

Author of requested content

Life of current edition

Up to 499

Main product

\section{Distribution}

Translation

Copies for the disabled?

Minor editing privileges?

Incidental promotional

use?

Currency

\section{Worldwide}

Original language of publication

No

Yes

No

CAD

\section{NEW WORK DETAILS}

\begin{tabular}{|c|c|c|c|}
\hline \multirow[t]{2}{*}{ Title } & Very long chain fatty acid & Institution name & University of Guelph \\
\hline & $\begin{array}{l}\text { metabolism is required in } \\
\text { acute myeloid leukemia }\end{array}$ & $\begin{array}{l}\text { Expected presentation } \\
\text { date }\end{array}$ & $2021-08-31$ \\
\hline Instructor name & Paul Spagnuolo & & \\
\hline
\end{tabular}

\section{ADDITIONAL DETAILS}




\section{REUSE CONTENT DETAILS}

$\begin{array}{ll}\begin{array}{l}\text { Title, description or } \\ \text { numeric reference of the } \\ \text { portion(s) }\end{array} & \begin{array}{l}\text { Very long chain fatty acid } \\ \text { metabolism is required in } \\ \text { acute myeloid leukemia }\end{array} \\ \begin{array}{l}\text { Editor of portion(s) } \\ \begin{array}{l}\text { Volume of serial or } \\ \text { monograph }\end{array}\end{array} & \text { Matthew Tcheng } \\ \begin{array}{l}\text { Page or page range of } \\ \text { portion }\end{array} & 75-165\end{array}$

Title of the
article/chapter the
portion is from

Issue, if republishing an article from a serial

Publication date of portion

\author{
The very long chain acyl- \\ COA dehydrogenase \\ (VLCAD) is the molecular \\ target of avocadyne \\ (AYNE) \\ American Society of \\ Hematology.; HighWire \\ Press.
}

N/A

2021-07-01

\section{PUBLISHER TERMS AND CONDITIONS}

Elsevier publishes Open Access articles in both its Open Access journals and via its Open Access articles option in subscription journals, for which an author selects a user license permitting certain types of reuse without permission. Before proceeding please check if the article is Open Access on http://www.sciencedirect.com and refer to the user license for the individual article. Any reuse not included in the user license terms will require permission. You must always fully and appropriately credit the author and source. If any part of the material to be used (for example, figures) has appeared in the Elsevier publication for which you are seeking permission, with credit or acknowledgement to another source it is the responsibility of the user to ensure their reuse complies with the terms and conditions determined by the rights holder. Please contact permissions@elsevier.com with any queries.

\section{Republication Terms and Conditions}

1. Description of Service; Defined Terms. This Republication License enables the User to obtain licenses for republication of one or more copyrighted works as described in detail on the relevant Order Confirmation (the "Work(s)"). Copyright Clearance Center, Inc. ("CCC") grants licenses through the Service on behalf of the rightsholder identified on the Order Confirmation (the "Rightsholder"). "Republication", as used herein, generally means the inclusion of a Work, in whole or in part, in a new work or works, also as described on the Order Confirmation. "User", as used herein, means the person or entity making such republication.

2. The terms set forth in the relevant Order Confirmation, and any terms set by the Rightsholder with respect to a particular Work, govern the terms of use of Works in connection with the Service. By using the Service, the person transacting for a republication license on behalf of the User represents and warrants that he/she/it (a) has been duly authorized by the User to accept, and hereby does accept, all such terms and conditions on behalf of User, and (b) shall inform User of all such terms and conditions. In the event such person is a "freelancer" or other third party independent of User and CCC, such party shall be deemed jointly a "User" for purposes of these terms and conditions. In any event, User shall be deemed to have accepted and agreed to all such terms and conditions if User republishes the Work in any fashion.

3. Scope of License; Limitations and Obligations.

3.1. All Works and all rights therein, including copyright rights, remain the sole and exclusive property of the Rightsholder. The license created by the exchange of an Order Confirmation (and/or any invoice) and payment by User of the full amount set forth on that document includes only those rights expressly set forth in the Order Confirmation and in these terms and conditions, and conveys no other rights in the Work(s) to User. All rights not expressly granted are hereby reserved. 
3.2. General Payment Terms: You may pay by credit card or through an account with us payable at the end of the month. If you and we agree that you may establish a standing account with CCC, then the following terms apply: Remit Payment to: Copyright Clearance Center, 29118 Network Place, Chicago, IL 60673-1291. Payments Due: Invoices are payable upon their delivery to you (or upon our notice to you that they are available to you for downloading). After 30 days, outstanding amounts will be subject to a service charge of $1-1 / 2 \%$ per month or, if less, the maximum rate allowed by applicable law. Unless otherwise specifically set forth in the Order Confirmation or in a separate written agreement signed by CCC, invoices are due and payable on "net 30" terms. While User may exercise the rights licensed immediately upon issuance of the Order Confirmation, the license is automatically revoked and is null and void, as if it had never been issued, if complete payment for the license is not received on a timely basis either from User directly or through a payment agent, such as a credit card company.

3.3. Unless otherwise provided in the Order Confirmation, any grant of rights to User (i) is "one-time" (including the editions and product family specified in the license), (ii) is non-exclusive and non-transferable and (iii) is subject to any and all limitations and restrictions (such as, but not limited to, limitations on duration of use or circulation) included in the Order Confirmation or invoice and/or in these terms and conditions. Upon completion of the licensed use, User shall either secure a new permission for further use of the Work(s) or immediately cease any new use of the Work(s) and shall render inaccessible (such as by deleting or by removing or severing links or other locators) any further copies of the Work (except for copies printed on paper in accordance with this license and still in User's stock at the end of such period).

3.4. In the event that the material for which a republication license is sought includes third party materials (such as photographs, illustrations, graphs, inserts and similar materials) which are identified in such material as having been used by permission, User is responsible for identifying, and seeking separate licenses (under this Service or otherwise) for, any of such third party materials; without a separate license, such third party materials may not be used.

3.5. Use of proper copyright notice for a Work is required as a condition of any license granted under the Service. Unless otherwise provided in the Order Confirmation, a proper copyright notice will read substantially as follows: "Republished with permission of [Rightsholder's name], from [Work's title, author, volume, edition number and year of copyright]; permission conveyed through Copyright Clearance Center, Inc. "Such notice must be provided in a reasonably legible font size and must be placed either immediately adjacent to the Work as used (for example, as part of a by-line or footnote but not as a separate electronic link) or in the place where substantially all other credits or notices for the new work containing the republished Work are located. Failure to include the required notice results in loss to the Rightsholder and CCC, and the User shall be liable to pay liquidated damages for each such failure equal to twice the use fee specified in the Order Confirmation, in addition to the use fee itself and any other fees and charges specified.

3.6. User may only make alterations to the Work if and as expressly set forth in the Order Confirmation. No Work may be used in any way that is defamatory, violates the rights of third parties (including such third parties' rights of copyright, privacy, publicity, or other tangible or intangible property), or is otherwise illegal, sexually explicit or obscene. In addition, User may not conjoin a Work with any other material that may result in damage to the reputation of the Rightsholder. User agrees to inform CCC if it becomes aware of any infringement of any rights in a Work and to cooperate with any reasonable request of CCC or the Rightsholder in connection therewith.

4. Indemnity. User hereby indemnifies and agrees to defend the Rightsholder and CCC, and their respective employees and directors, against all claims, liability, damages, costs and expenses, including legal fees and expenses, arising out of any use of a Work beyond the scope of the rights granted herein, or any use of a Work which has been altered in any unauthorized way by User, including claims of defamation or infringement of rights of copyright, publicity, privacy or other tangible or intangible property.

5. Limitation of Liability. UNDER NO CIRCUMSTANCES WILL CCC OR THE RIGHTSHOLDER BE LIABLE FOR ANY DIRECT, INDIRECT, CONSEQUENTIAL OR INCIDENTAL DAMAGES (INCLUDING WITHOUT LIMITATION DAMAGES FOR LOSS OF BUSINESS PROFITS OR INFORMATION, OR FOR BUSINESS INTERRUPTION) ARISING OUT OF THE USE OR INABILITY 
TO USE A WORK, EVEN IF ONE OF THEM HAS BEEN ADVISED OF THE POSSIBILITY OF SUCH DAMAGES. In any event, the total liability of the Rightsholder and CCC (including their respective employees and directors) shall not exceed the total amount actually paid by User for this license. User assumes full liability for the actions and omissions of its principals, employees, agents, affiliates, successors and assigns.

6. Limited Warranties. THE WORK(S) AND RIGHT(S) ARE PROVIDED "AS IS". CCC HAS THE RIGHT TO GRANT TO USER THE RIGHTS GRANTED IN THE ORDER CONFIRMATION DOCUMENT. CCC AND THE RIGHTSHOLDER DISCLAIM ALL OTHER WARRANTIES RELATING TO THE WORK(S) AND RIGHT(S), EITHER EXPRESS OR IMPLIED, INCLUDING WITHOUT LIMITATION IMPLIED WARRANTIES OF MERCHANTABILITY OR FITNESS FOR A PARTICULAR PURPOSE. ADDITIONAL RIGHTS MAY BE REQUIRED TO USE ILLUSTRATIONS, GRAPHS, PHOTOGRAPHS, ABSTRACTS, INSERTS OR OTHER PORTIONS OF THE WORK (AS OPPOSED TO THE ENTIRE WORK) IN A MANNER CONTEMPLATED BY USER; USER UNDERSTANDS AND AGREES THAT NEITHER CCC NOR THE RIGHTSHOLDER MAY HAVE SUCH ADDITIONAL RIGHTS TO GRANT.

7. Effect of Breach. Any failure by User to pay any amount when due, or any use by User of a Work beyond the scope of the license set forth in the Order Confirmation and/or these terms and conditions, shall be a material breach of the license created by the Order Confirmation and these terms and conditions. Any breach not cured within 30 days of written notice thereof shall result in immediate termination of such license without further notice. Any unauthorized (but licensable) use of a Work that is terminated immediately upon notice thereof may be liquidated by payment of the Rightsholder's ordinary license price therefor; any unauthorized (and unlicensable) use that is not terminated immediately for any reason (including, for example, because materials containing the Work cannot reasonably be recalled) will be subject to all remedies available at law or in equity, but in no event to a payment of less than three times the Rightsholder's ordinary license price for the most closely analogous licensable use plus Rightsholder's and/or CCC's costs and expenses incurred in collecting such payment.

8. Miscellaneous.

8.1. User acknowledges that CCC may, from time to time, make changes or additions to the Service or to these terms and conditions, and CCC reserves the right to send notice to the User by electronic mail or otherwise for the purposes of notifying User of such changes or additions; provided that any such changes or additions shall not apply to permissions already secured and paid for.

8.2. Use of User-related information collected through the Service is governed by CCC's privacy policy, available online here:https://marketplace.copyright.com/rs-ui-web/mp/privacy-policy

8.3. The licensing transaction described in the Order Confirmation is personal to User. Therefore, User may not assign or transfer to any other person (whether a natural person or an organization of any kind) the license created by the Order Confirmation and these terms and conditions or any rights granted hereunder; provided, however, that User may assign such license in its entirety on written notice to CCC in the event of a transfer of all or substantially all of User's rights in the new material which includes the Work(s) licensed under this Service.

8.4. No amendment or waiver of any terms is binding unless set forth in writing and signed by the parties. The Rightsholder and CCC hereby object to any terms contained in any writing prepared by the User or its principals, employees, agents or affiliates and purporting to govern or otherwise relate to the licensing transaction described in the Order Confirmation, which terms are in any way inconsistent with any terms set forth in the Order Confirmation and/or in these terms and conditions or CCC's standard operating procedures, whether such writing is prepared prior to, simultaneously with or subsequent to the Order Confirmation, and whether such writing appears on a copy of the Order Confirmation or in a separate instrument.

8.5. The licensing transaction described in the Order Confirmation document shall be governed by and construed under the law of the State of New York, USA, without regard to the principles thereof of conflicts of law. Any case, controversy, suit, action, or proceeding arising out of, in connection with, or related to such licensing transaction shall be brought, at CCC's sole discretion, in any federal or state court located in the County of New York, State of New York, USA, or in any federal or state court whose geographical jurisdiction covers the location of the Rightsholder set forth in the Order Confirmation. The parties 
expressly submit to the personal jurisdiction and venue of each such federal or state court.If you have any comments or questions about the Service or Copyright Clearance Center, please contact us at 978-750-8400 or send an e-mail to support@copyright.com.

v 1.1 


\title{
9.2.3 Chapter 6
}

Firefox

https://outlook.live.com/mail/inbox/id/AQMkADAwATc3AGZmAGU...

RE: Requesting permission to use figures in Food \& Function: FO-

ART-03-2021-000693.R1 in a thesis

\author{
FOOD (shared) <FOOD@rsc.org> \\ Thu 2021-05-20 8:10 AM \\ To: 'matt tcheng' <mtcheng416@msn.com> \\ Cc: 'Paul Spagnuolo' < paul.spagnuolo@uoguelph.ca> \\ Dear Matthew
}

Thank you for your email. It is fine for you to use the figures from your own paper for your own thesis. You can find a flowchart for figure permissions at https://www.rsc.org/journals-books-databases/journal-authorsreviewers/licences-copyright-permissions/\#reuserscmaterial that you may find useful.

With thanks,

Andrea Whiteside

Publishing Assistant - Journals

Royal Society of Chemistry

Thomas Graham House,

Science Park, Milton Road

Cambridge, CB4 OWF, UK

www.rsc.org

From: matt tcheng <mtcheng416@msn.com>

Sent: 19 May 2021 15:02

To: FOOD (shared) <FOOD@rsc.org>

Cc: Paul Spagnuolo <paul.spagnuolo@uoguelph.ca>

Subject: Requesting permission to use figures in Food \& Function: FO-ART-03-2021-000693.R1 in a thesis

Hello Food and Function,

My name is Matthew Tcheng, I am the first author on Food \& Function: FO-ART-03-2021-000693.R1, a recently accepted article. I am requesting permission to use all unmodifed figures (Figures 1-4) and supplementary figures (suppl. fig. 1-2) from this article for my graduate thesis. Since there does not seem to be an official webpage to request permission for this article yet, please let me know any additional details you require, if this is possible, and of any potential charges.

Paul Spagnuolo, my graduate supervisor and the corresponding author of this article, has been cc'd on this email.

-Matthew 